\title{
Die Wissenschaft
}

\section{Sammlung naturwissenschaftlicher und}

\section{mathematischer Monographien}

\section{$=$ Heft $44=$}

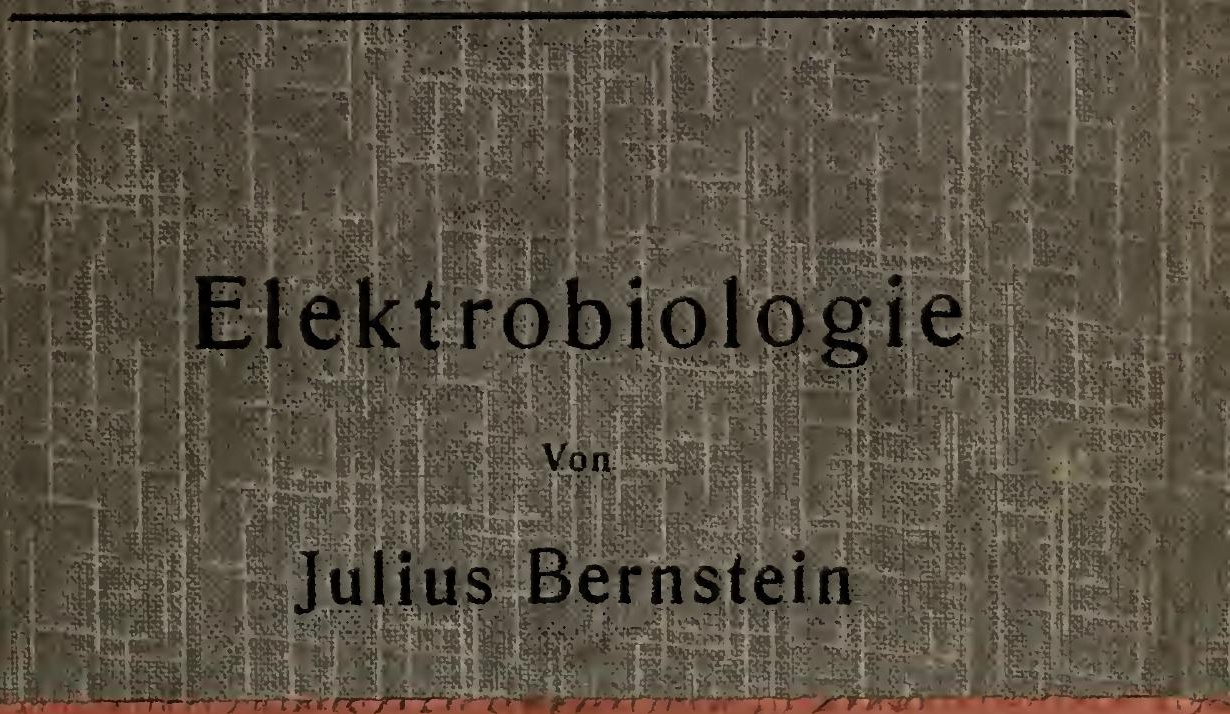




\section{m. z. Collent}

\section{Buffolo, 1421.}

\section{A N K Ü N D I G U N G.}

In dem vorliegenden Bande der "Wissenschaft" werden die in den Organismen stattfindenden elektrischen Vorgänge und ihre Bedeutung für das Leben eingehend behandelt. Die Schrift dürfte einem größeren Leserkreise insofern willkommen sein, als das darin dargestellte Gebiet des Wissens weder in den Lehrbüchern der Physik oder der Elektrochemie noch in denen der Physiologie in der Vollständigkeit und in dem Zusammenhange berücksichtigt werden kann, wie es zum Verständnis des Gegenstandes erforderlich ist. Daber wird dieses Buch das Interesse aller naturwissenschaftlich gebildeten Leser, welche sich eine Kenntnis jener hochinteressanten Lebensvorgänge verschaffen wollen, beanspruchen dürfen. Zugleich wird es, da der Verfasser von den modernen Lehren der physikalischen Chemie ausgehend das Gebiet der Bioelektrizität in ihm neu zu begründen und durch eigene Forschungen zu erweitern strebt, auch dem Physiologen Neues bieten.

Braunschweig, in Oktober 1912.

Friedr. Vieweg \& Soln. 
Verlag von Friedr. Vieweg \& Sohn in Braunschweig

\section{DIE WISSENSCHAFT}

Sammlunn nafurwissenschaillicher und mathemalischer Monographien.

Neueste Bände:

32. Heft. Jesionek, Dr. Albert, Lichtbiologie. Die experimentellen Grundlagen der modernen Lichtbehandlung. 1910. M 4,- , in Lnwdbd. Nh 4,80.

33. Heft. Dessau, Prof. Dr. Bernhard, Die physikalischchemischen Eigenschaften der Legierungen. Mit $82 \mathrm{Ab}$ bildungen im Text und auf 3 Tafeln. 1910.

M 7,-, in Lnwdbd. M 8,- .

34. Heft. Pohl, Dr. Robert, Die elektrische Fernübertragung von Bildern. Mit 25 Abbildungen. 1910.

N1. 1,80, in Lnwdbd. M 2,50.

35. Heft. Baedeker, Prof. Dr. K., Die elektrischen Erscheinungen in metallischen Leitern. (Leitung, Thermoelektrizität, Galvanomagnetische Effekte, Optik.) Mit 25 Abbildungen. 1911. $\quad$ M 4,-, in Lnwdbd. NH 4,80.

36. Heft. Scheel, Prof. Dr. Karl, Grundlagen der praktischen Metronomie. Mit 39 Abbildungen. 1911.

M 5,20, in Lnwdbd. M 6,- .

37. Heft. Günther, Prof. Dr. Sigmund, Vergleichende Mondund Erdkunde. Mit 23 Abbild. im Text und 4 Tafeln. 1911.

$/$ 5,-, in Lnwdbd. M 5,80.

38. Heft. Laue, Dr. M., Das Relativitätsprinzip. Mit 14 Abbildungen. 1911.

Ml 6,50, in Lnwdbd. the 7,20.

39. Heft. Müller, Aloys, Das Problem des absoluten Raumes und seine Beziehung zum allgemeinen Raumproblem. 1911. M. 4,-, in Lnwdbd. M 4,80. 
Verlag von Friedr. Vieweg \& Sohn in Braunschweig

\section{Die Wissenschaft Sammlung naturuissenschattlicher

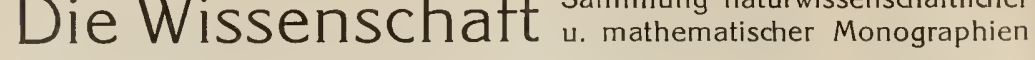

Neueste Bände:

40. Heft. Schmidt, Ingenieur Fr., Die Leuchtgaserzeugung u. die moderne Gasbeleuchtung (Preßgasbeleuchtung usw.). Mit 63 Abbild. 1911. M 2,50, in Lnwdbd. Nh 3,20.

41. Heft. Lodge, Sir Oliver, Der Weltäther. Übersetzt von Hilde Barkhausen. Mit 17 Textabbildungen und einer Tafel. 1911. M 3,-, in Lnwdbd. Ml 3,60.

42. Heft. Lampa, Prof. Dr. Otto, Wechselstromversuche. Mit 54 Abbild. 1911. M 5,-, in Lnwdbd. M 5,80.

43. Heft. Markau, Dr. K., Die Telephonie ohne Draht. Mit 103 Abbild. 1912. M 4,50, in Lnwdbd. M 5,20.

44. Heft. Bernstein, Julius, Elektrobiologie. Die Lehre von den elektrischen Vorgängen im Organismus auf moderner Grundlage dargestellt. Mit 62 Abbildungen. 1912. th 6,- , in Lnwdbd. d6 6,80.

45. Heft. Pohl, Dr. Robert, Die Physik der Röntgenstrahlen. Mit 72 Abbildungen im Text und auf einer Tafel. 1912. M 5,-, in Lnwdbd. M 5,80.

Demnächst werden erscheinen:

Physikalische Grundlagen der Elektrotechnik. Von F. Martens (2 Bde.).

Das Leuchten der Gase und Dämpfe. Von Professor Dr. H. Konen.

Mimikry. Von Prof. Dr. Jacobi.

Die Arbeitsbiene. Von Prof. Dr. von Buttel-Reepen.

Ein vollständiges Verzeichnis der Sammlung mit Inhaltsangabe der $\therefore$ einzelnen Bände befindet sich am Schluß dieses Buches :: 


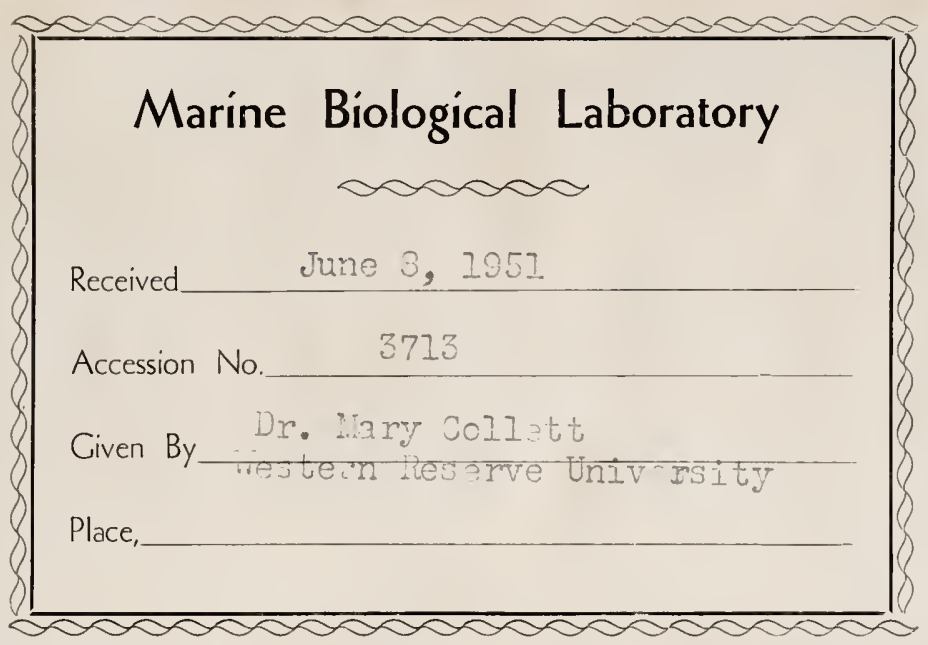




\section{DIE WISSENSCHAFT}

SAMMLUNG

NATURWISSENSCHAFTLICHER UND MATHEMATISCHER

MONOGRAPHIEN

\section{VIERUNIVIERZIGSTES HEFT}

\section{ELEKTROBIOLOGIE}

DIE LEHRE VON DEN ELEKTRISCHEN VORGÄNGEN IM ORGANISHUS AUF MODERNER GRUNDLAGE DARGESTELLT

$\nabla 0 \mathrm{~N}$ JULIUS BERNSTEIN

IIT 62 ABBILDUNGEN IM TEXT

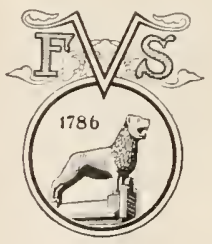

B R A U N S C H W E I G

DRUCK UND VERLAG VON FRIEDR. VIEWEG \& SOHN 


\title{
ELEKTROBIOLOGIE
}

\author{
DIE LEHRE VON DEN
}

ELEKTRISCHEN VORGÄNGEN IM ORGANISMUS AUF MUDERNER GRUNDLAGE DARGESTELLT

$\operatorname{VON}$

\section{JULIUS BERNSTEIN}

o. Ö. PROFESSOR DER PHYSIOLOGIE AN DER UNIVERSITÄT

HALLE A. S.

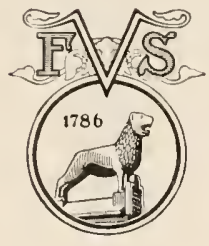

B RA U N SCH W E I G

DRUCK UND VERLAG VON FRIEDR. VIEWEG \& SOHN 
Alle Rechte, namentlich dasjenige der Übersetzung in fremde Sprachen, vorbehalten.

Copyright, 1912, by Friedr. Vieweg \& Sohn, Braunschweig, Germany.

\section{$37 / 3$}




\section{VOR IV ORT.}

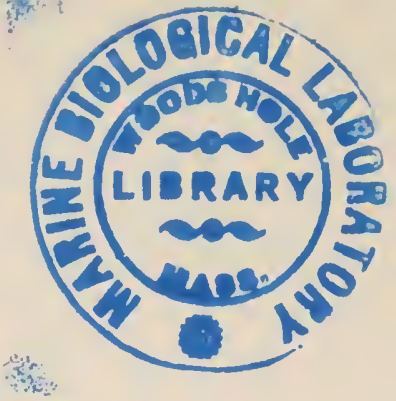

Indem ich dieses Buch der Öffentlichkeit übergebe, möchte ich über den Inhalt desselben und die Darstellung des darin behandelten Stoffes einiges vorausschicken. Bereits im Jahre 1904 wurde ich aufgefordert, für die Sammlung, in welcher dieses Buch erscheint, ein Werk ïber „die Bioelektrizität uach modernen Anschauungen" zu schreiben. Wenn ich jetzt erst dieser Aufforderung Genüge leiste, so möchte ich zu meiner Rechtfertigung das Horazische "Nonum prematur in annum" für mich in Anspruch nehmen. Nachdem ich im Jahre 1902 durch Aufstellung der "Membrantheorie der bioelektrischen Ströme" dazu-beigetragen hatte, einer nenen Richtung in der Elektrophysiologie freie Bahn zu schaffen, bedurfte es zur Festigung dieser Theorie und Weiterentwickelung derselben noch vieler Spezialuntersuchungen, mit denen ich und meine Schiiler in den letzten zehn Jahren beschäftigt waren. Dazu kam, daß sehr bald das Interesse für die elektrophysiologische Forschung durch Ausbildung der graphischen Untersuchungsmethoden mit Hilfe des Kapillarelektrometers und des Saitengalvanometer's einen neuen Aufschwung nahm. So war in dieser Zeit in dem Gebiete der Elektrophysiologie vieles in beständigem Fluß, und erst jetzt ist ein Zeitpunkt eingetreten, in welchem ein vorläufiger Abschluß dieses Gebietes möglich erscheint. 
Dieses Buch soll nun hauptsächlich die Aufgabe erfiillen, weiteren Kreisen der für physiologische Forschung sich interessierenden Leser, wie dem Mediziner, dem Physiker, Chemiker, Techniker und allen naturwissenschaftlich Gebildeten die Grundlagen der modernen Lehre von der Bioelektrizität zur Kenntnis zu bringen. Außerdem aber hat die stetige experimentelle Arbeit, mit der ich bis in die letzte Zeit zur Ausarbeitung dieser Lehre beschäftigt war, es mit sich gebracht, daß in diesem Buche auch eine Reihe neuer Tatsachen und daraus geschlossener Folgerungen zum ersten Male veröffentlicht werden. Daher hoffe ich, daß auch meine Fachkollegen diesem Buche ihr Interesse zuwenden werden.

Die Begrïndung des Titels dieses Buches als „Elektrobiologie", welche gegenüber dem bisher üblichen Ausdruck "Elektrophysiologie" einen erweiterten Begriff dieses Gebietes der Biologie bezeichnen soll, wird man am Schlusse des Buches in der "Zusammenfassung und Schlußbetrachtung“ finden.

$$
\text { Halle a. S., im August } 1912 .
$$

\section{Julius Bernstein.}


Viertes Kapitel.

Fortsetzung und elektrische Vorgïinge in anderen Organen .

Aktionsströme und Reizwelle des Nerven. Absolute Größe derselben. Reizwellen der Nerven im lebenden Körper. Elektrische Reaktion der 'Ventralorgane. Aktionsströme des Herzens. Das Elektrokardiogramm. Glatte Muskelfasern und ihre elektrischen Eigenschaften. Die Ströne der Netzhaut, Lichtreaktion derselben.

\section{Fünftes Ḱapitel.}

Die Membrantheorie ........

Semipermeable Membranen nach Pfeffer, elektrische Eigenschaften derselben nach W. Ostwald. Versuche von Oker-Blom, Untersuchungen von J. Bernstein über das Verhalten des Muskelstromes zur absoluten Temperatur. Die elektrische Membrantheorie. Das Membranpotential hat seinen Sitz am Längsschnitt. Präexistenz und Alteration. Versuche von Höber ïber Einwirkung von Elektrolyten. Verhalten des Nervenstromes zur absoluten Temperatur und Membranpotential des Nerven. Die negative Schwankung und die Alitionsströme nach der Membrantheorie. Wahrer Wert des Membranpotentials.

\section{Sechstes Kapitel.}

Die elektrischen Organe . . . . . . . .

Bau der elektrischen Organe. Richtung und zeitlicher Verlauf des Schlages. Kraft desselben. 'Temperaturkoeffizient der Kraft. Das elektrische Organ, eine Konzentrationskette. Anwendung der Membrantheorie. 'Thermodynamische Untersuchung des Organs von Bernstein und 'Tschermak. Chemische Zusammensetzung.

Siebentes Kapitel.

Innere Polarisation und elektrische Reizung . . .

Elektrotonus und innere Polarisation der Nerven und Muskeln. Keruleiter nach I. Hermann. Membranpolarisation. Gesetz der polaren Erregung des Kat- und Anelektrotonus nach der Membrantheorie. Energetische Auffassung der Reizbarkeit und physiologischen Leistung. Gesetz der elektrischen Reizung. Formeln von du Bois-Reymond, Hoorweg, G. We is s, Nernst. Allgemeinere Formel, Deutung nach der Membrantheorie. 
Achtes Kapitel.

Haut- und Driisenströme. Ihre Bedeutung fiir die Sekretion Seite und Resorption. Die LIektroosmose. Elektroosmotische

Membrantheorie....... . 151

Ströme der Froschhaut und anderer Häıte. Die Sekretionszelle. Negative und positive Schwankung bei der Reizung. Deutung nach der Membrantheorie. Die Resorptionszellen der äußeren Haut und der Schleimhäute des Darmes. Die physikalische Elektroosmose. Elektroosmotische Membrantheorie der Sekretion und liesorption. Llektroosmotische Eigenscliaft semipermeabler Membranen.

Neuntes Kapitel.

Urspringliche physiologische Bedeutung des Membranpotentials der Zellen. Die Wasserbindung in den Zellen. Genese der elektrischen Eigenschaften der Organe.

Die Pflanzenströme . . . . . . .

Die Wasserbindung in den Zellen durch osmotischen Druck und durch das Membranpotential. Versuche über die Verdunstung lebender und toter Membranen und Organe; Versuche an der Froschliaut, an P'flanzenblättern, an Muskeln. Die lebende Zelle bindet das Wassel stärker als die tote. Erklärung nach der elektroosmotischen Membrantheorie. Die binelektrischen Ströme und die Reizbewegungen an Pflanzen.

Zehntes Kapitel.

Die Elektrokinese. . . . . . . .

Verhalten der Kolloide im Potentialgrefälle. Theorie der Ausfällungen und Gerinnungen derselben. Die Suspensionskolloide und hydrophilen Kolloide. Das Verhalten von suspendierten Zellen im l'otentialgefälle. Die Bewegung der Kernfäden bei der Zellteilung (Karyokinese) als Elektrokinese.

Zusammenfassung und Sclilußbetrachtung . . . . . . 196

Anhang. . . . . . . . . . . . . . . . 206

Sach-und Namenregister. . . . . . . . . . 213

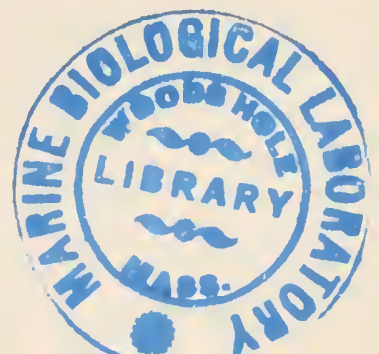





\section{Erstes Kapitel. \\ Historisches und Einleitung.}

Nachdem Galvani in Jahre 1786 beobachtet hatte, dal Zuckungen in den Muskeln auftreten, wenn man dieselben oder ihre Nerven mit einem Bogen zweier Metalle, z. B. aus Zink und Kupfer, berührt, glaubte man eine Zeitlang damit das Vorhandensein einer tierischen Elektrizität entdeckt zu haben, die in diesen Organen als Lebenskraft wirke. Bei Gelegenheit eines Gewitters stieg er auf die Terrasse seines Hauses in Bologna, um den Einfluß der atmosphärischen Elektrizität auf tierische Organe zu untersuchen, und hing an dem eisernen Gitter der Terrasse mit einem Metallhaken frisch präparierte Froschschenkel auf. Die bei zufälligen Berührungen der Schenkel mit beiden Metallen auftretenden Zuckungen waren der denkwürdige Ausgangspunkt einer großen Reihe von Untersuchungen, welche zu den bekannten Entdeckungen Voltas führten. Obgleich dieser überzeugend nachwies, daß jene Zuckungen nicht die Folge einer Muskel- und Nervenelektrizität seien, sondern durch den Strom entstehen, den die Metalle mit den Organen als feuchten Leitern erzengen, so hielt Galvani doch au seiner Überzengung fest. Aber auch die von ihm beobachteten Zuckungen, welche bei Anlegung eines Bogens aus ein und demselben Metall entstehen können, erklärte Volta ebenfalls durch die Ungleichartigkeiten desselben Motalles an den berührten Stellen, was sich auch späterhin vollkommen bestätigte. Nichtsdestoweniger fand Galvani bei seinen rastlosen Bemühungen, seine Theorie zu stützen, einen merkwürdigen Versuch, die "Zuckung ohne Metalle", welche allerdings, wio sich erst später, namentlich durch die Untersuchungen du BoisReymonds herausstellte, ein wirklicher Beweis für das Vor- 
handensein eines physiologischen Stromes des Muskels gewesen ist. Wenn man nämlich den Nerven eines Froschschenkels, Nervus ischiadicus, auf die Hinterfläche des Wadenmuskels, Musculus gastrocnemius, so auffallen läßt, daß er Bauch und untere Sehne desselben, die Achillessehne, in größerer Ausdehnung plötzlich berührt, so entsteht häufig eine Zuckung des Muskels. Sie ist nicht die Folge einer mechanischen Erschütterung des Nerven beim Auffallen, denn sie entsteht nicht beim Auffallen des Nerven auf eine andere harte Unterlage, z. B. eine Glasplatte ${ }^{2}$ ). Der Strom des Muskels ergießt sich vielmehr in dem Moment der Berührung durch den Nerven und erzeugt so eine Reizung desselben. Doch nicht immer tritt dieser Erfolg ein. Es ist dies, wie wir sehen werden, wesentlich rom Zustande des Muskels abhängig. Dagegen treten Zuckungen konstant auf, wenn man, wie Alex. v. Humboldt sah, den Nerven eines Schenkels an die Oberfläche und den Querschnitt eines lebenden Muskels anlegt, ebenfalls ein Beweis für das Vorhandensein eines bioelektrischen Stromes. Galvani stellte sich vor, daß der Muskel und Nerv sich wie eine geladene Leidener Flasche verhielte, daß die Oberfläche des Muskels mit der einen, das Innere desselben mit der anderen Elektrizität geladen seien, und daß der Nerv, der in das Innere eintritt, gleichsam den Konduktor bilde. Nachdem Nobili bald nach der Erfindung des Multiplikators durch Schweigger und Poggendorff denselben durch Anwendung des astatischen Nadelpaares empfindlicher gemacht hatte, beobachtete er an den enthäuteten unteren Gliedmaßen des eben getöteten Frosches einen von den Zehen nach der Wirbelsäule fließenden Strom, den er den Froschstrom nannte, und dann zeigte Matteucci, daß auch der einzelne frische Muskel einen Strom erzeugt, wenn man seine Oberfläche mit einem Querschnitt verbindet. (Er behauptete, daß die Oberfläche eines Iuskels positive, das ganze Innere desselben negative Spannung besäße.? Aber erst durch die ausgezeichneten und umfassenden Untersuchungen Emil du Bois-Reymonds ${ }^{2}$ ) ist das Gesetz des Muskelstromes festgestellt worden.

$\left.{ }^{1}\right)$ Beim Auffallen auf eine Metallplatte können Zuckungen durch Ungleichartigkeiten der berührten Netalloberfläche auftreten.

2) Untersuchungen über tierische Elektrizität, I. Bd. 1848; II, 1,1849 , II, 2, 1884 . 
Lange Zeit war die Ableitung der Ströme von den tierischen Organen eine unvollkommene, weil polarisierbare. Man tauchte Platinelektroden in konzentrierte $\mathrm{Cl} \mathrm{Na}$ - Lösung und brachte zwischen die Organe und ClNa-Lösung indifferente Flüssigkeiten, wie Eiereiweiß, welche die Organe nicht angriffen. Die Ungleichartigkeit und Polarisierbarkeit derselben stellten aber der Untersuchung viele Schwierigkeiten entgegen, da schwache Ströme durch Polarisation der Platinplatten aufgehoben werden. Erst später konstruierte du Bois-Reymond die jetzt gebränchlichen unpolarisierbaren Elektroden, welche aus amalgamiertem Zink in kon-

Fig. 1.

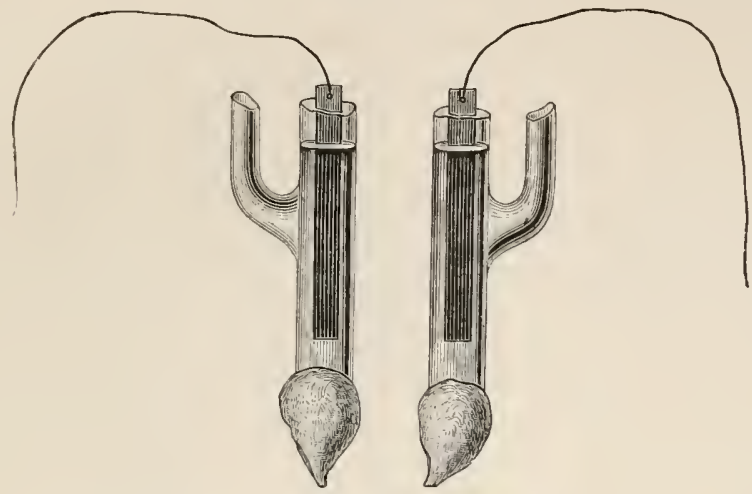

Unpolarisierbare Flektroden von E. du Bois-Reymond.

zentrierter $\mathrm{ZnSO}_{4}$-Lösung und physiologischer $\mathrm{Cl} \mathrm{Na-Lösung,}$ 0,6 bis 0,8 Proz., welche die Organe nicht angreift, zusammengesetzt sind. Fig. 1 gibt die gebräuchliche Form derselben wieder. Sie bestehen aus zwei platten Röhrchen, welche unten mit einer Tonspitze aus reinem Modellierton geschlossen werden, der mit physiologischer Kochsalzlösung getränkt ist. In die Röhrchen wird die $\mathrm{ZnSO}_{4}$ - Lösung eingefüllt, in welche die Zinkelektroden eintauchen. Diese Elektroden sind unpolarisierbar, da sie in bezug auf das Kation $\mathrm{Zn}^{++}$umkelırbar sind. $\mathrm{Zn}$ wandert von der Anode zur Kathode und scheidet sich in letzterer ab, während $\mathrm{SO}_{4}$ nach der Anode wandert und dort $\mathrm{Zn}$ auflöst. Es ändern sich nur die Konzentrationen der Zn-Lösungen an den Elektroden durch die verhältnismäßig schwachen Ströme in sehr geringem 
Maße, und diese wird durch den entgegengesetzten gleich starken Strom wieder rückgängig gemacht.

Später sind auch die von Helmboltz und Ostwald zu physikalischen Zwecken angegebenen Quecksilber-Kalomelelektroden in Gebrauch genommen worden. Dieselben bestehen aus der Kombination von Quecksilber, welches wit Kalomelpulver, $\mathrm{Hg}_{2} \mathrm{Cl}_{2}$, bestreut wird, worüber die Lösung eines Alkalichlorids geschichtet wird nach dem Schema:

$-\cdots \mathrm{Hg}_{2}, \overparen{\mathrm{Hg}_{2}-\mathrm{Hg}_{2}} \mathrm{Cl}_{2}-\mathrm{Hg}_{2} \mathrm{Cl}_{2}-\mathrm{Na}_{2} \mathrm{Cl}_{2}-\mathrm{Na}_{2} \mathrm{Cl}_{2}-\mathrm{H}_{2} \mathrm{Cl}_{2}-\overparen{\mathrm{H}}_{2} \mathrm{Cl}_{2}-\mathrm{Hg}_{2}, \mathrm{Hg}_{2} \cdots+\leftarrow$

Gelit der Strom in der Richtung des Pfeiles hindurch, so erkennt man, daß sich an der Anode, nach welcher $\mathrm{Cl}_{2}$ wandert,

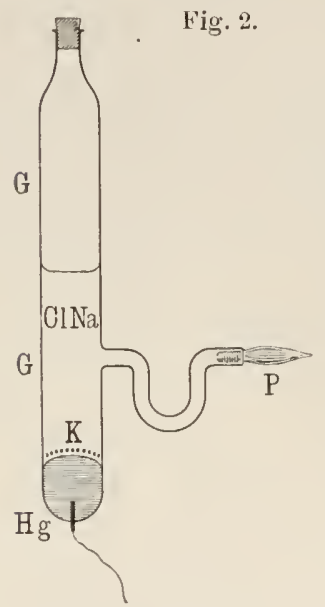

Quecksilber-KalomelElektroden nach $0 \mathrm{ker}-\mathrm{Bl}$ om. $G G$ Glasgefäß, $H g$ Quecksilber, K Kalomel, $C l N$ a physiologische

ClNa-Lösung, $P$ Pinsel zur Ableitung. $\mathrm{Hg}_{2} \mathrm{Cl}_{2}$ bildet, und an der Kathode, nach welcher $\mathrm{Na}_{2}$ wandert, sich $\mathrm{Hg}_{2}$ und $\mathrm{Hg}_{2} \mathrm{Cl}_{2}$ ausscheidet. $\mathrm{Hg}_{2} \mathrm{Cl}_{2}$ wirkt als Depolarisator. Diese Elektroden sind daher unpolarisierbar und in bezug auf das Anion $\left(\mathrm{Cl}^{-}\right)$umkehrbar. Fig. 2 zeigt die von Oker-Blom zu physiologischen Zwecken angegebene Form der $\mathrm{Hg}$ Kalomelelektroden.

Man benutzt bei beiden Elektrodenarten zur. Verbindung mit den Organen die physiologische $\mathrm{ClNa}$-Lösung, weil diese die Organe nicht augreift, da sie denselben osmotischen Druck wie die Flüssigkeiten der Organismen, das Blut, die Lymphe und Gewebssäfte besitzt, und weil in diesen das $\mathrm{Cl} \mathrm{Na}$ nebst geringen Mengen anderer Salze in nahezu derselben Konzentration enthalten ist. In der physiologischen Kochsalzlösung bleiben daher die Nerveu und Muskeln lange Zeit in lebendem Zustande gut reizbar und funktionsfähig. Die geringen Potentialdifferenzen zwischen Zinklösung oder $\mathrm{Hg}_{2} \mathrm{Cl}_{2}$ - und $\mathrm{ClNa}$-Lösung heben sich auf beiden Seiten auf, und die Gewebsflüssigkeiten, mit denen die Organe imbibiert und befeuchtet sind, können an beiden Elektroden mit der physiologischen Kochsalzlösung in bezug auf die in ihnen enthaltenen Elektrolyte als gleich angesehen werden. 
Das von E. du Bois-Rey mond gefundene Gesetz des Muskelstromes kanu nur an einem regelmäßig gestalteteu Muskel, der aus gleich langen parallelen Fasern besteht, festgestellt werden. Begrenzt man denselben durch zwei künstliche Querschnitte, indem man die meist unregelmäßig gestalteten Sehnenenden mit dem Messer abtremnt, wie Fig. 3 zeigt, so erbält man folgendes liesultat: Die Längsoberfläche des Muskels, welche man den "natürlichen Längsschnitt" nennt, zeigt positive, und der künstliche Querschuitt zeigt negative Spannung, oder der Längsschnitt ist der positive, der Querschnitt der negative Pol des Muskels, den wir als eine physiologische Kette betrachten könuen. Der abgeleitete Strom fließt, wie die Fig. 3 zeigt, in einem abgeleiteten Galvanometerki'eise von dem Längsschnitt $L$ zum Querschnitt $Q$, und in dern Muskel selbst vom Querschnitt zum Längsschnitt. Mau hat diesen Strom somit áuch zweckmäßig den "Längsquerschnitstrom"1) genannt. Da zur Er-

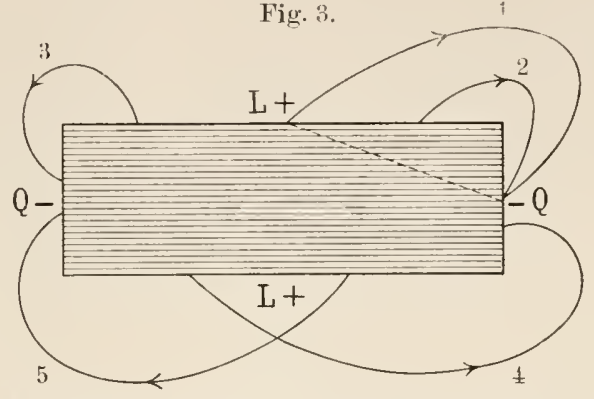

Muskelströme zwischen Längsschnitt und Querschnitt.

zengung dieses Stromes eine Verletzung der Muskelfasern in ihrer Kontinuität erforderlich ist, so hat man die durch Verletzung entstehenden Ströme auch allgemein "Läsionsströme" genannt. Man findet daher, daß eine durch Quetschung, Ätzung oder stärkere Erhitzung abgetötete Stelle des Muskels sich negativ gegen die lebende Partie desselben verhält, und zwar im allgemeinen ebenso stark, wie der künstliche Querschnitt gegen den Längsschnitt. Man

1) L. Hermann hat sich in seinen ersten Arbeiten über diesen Gegenstand des Ausdruckes "Demarkationsstrom" bedient, hat ihn aber später zugunsten des obigen fallen lassen. Anch ich muß ihn verwerfen, da in ihm schon die Voraussetzung einer Thenrie liegt. Demarkation bedeutet bekanntlich in der Pathologie den Prozeß Aer Abgrenzung des lebenden vom toten Gewebe beim Vorgange der Heilung (s. weiter unten Theorie). 
kann statt mit dem Messer auch durch Erwärmen auf 45 bis $50^{\circ}$ einen sogenaunten "Wärmęuerschnitt" an dem Muskel anbringen.

Untersucht man den regelmäligen, mit zwei künstlichen senkrechten Querschnitten begrenzten Muskel genauer, indem man verschiedene Punkte des natürlichen Längsschnittes und künstlichen Querschnittes ableitet, so findet man, dab jede beliebige Stelle des Längsschnittes und Querschnittes einen Strom liefert, aber von ungleicher Kraft. Die größte Potentialdillerenz herrscht zwischen den Mittelpunkten des Längsschnittes und dem Mittelpunkt des (kreisförmig gedachten) Querschnittes; dieselbe beträgt bei frischen, kräftigen Froschmuskeln 0,04 bis

Fig. 4.

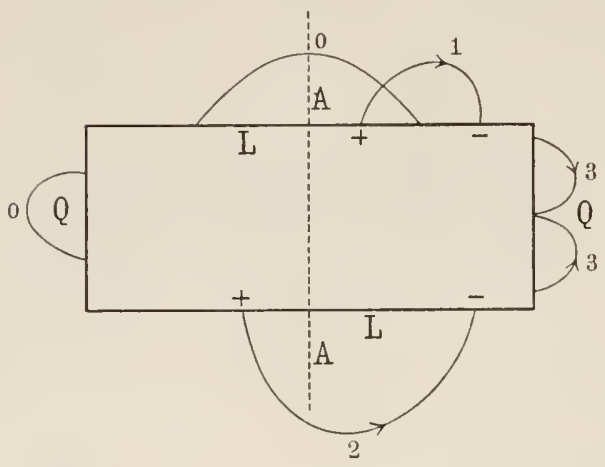

0,08 Volt. Von diesen Stellen aus nehmen nach beiden Seiten die Potentialdifferenzen ab. Daraus folgt, da $B$ auch Punkte des Längsschnittes und solche des Querschnittes schwächere Ströme geben müssen, wie Fig. 4 zeigt. Legt man senkrecht durch die Mitte des Muskels eine Ebene $A A$, den "Äquator", so sind alle dem Äquator wäheren Punkte positiv gegen die entfernteren, zwei symmetrisch zum Äquator gelegene Punkte dagegen geben keinen Strom. Ebenso zeigen größere Querschnitte schwache Ströme, indem die dem Mittelpunkt näheren Stellen negativ gegen entferutere sind.

Nicht nur der natürliche, sondern auch der künstliche Längsschnitt, den man durch Längsspaltung eines Muskels erhält, zeigt gegen den künstlichen Querschnitt dieselbe Potentialdifferenz. Zerfasert man einen Muskel in möglichst dünne lange Bündel von Mluskelfasern, so zeigen auch diese dieselben Eigenschaften, abgesehen davon, daß bei dieser Präparation leicht Verletzungen auftreten können. Wäre es daher möglich, eine einzelne Mruskelfaser in unverletztem, lebendem Zustande zu präparieren, so unterläge es hiernach keinem Zweifel, daß auch diese 
einen Strom in demselben Sinne geben würde. Hiermit war von du Bois-Reymond die Matteuccische Ansicht, daß das ganze Innere des Muskels negatir gegen die Oberfläche wäre, widerlegt. Die Ströme des ganzen Muskels setzen sich aus deneu der einzelnen M uskelfasernzusammen. Die Potentiale des ganzen Muskels sind aber denen der einzelnen Fasern gleich, weun diese alle sich in gleichem Zustande befinden. Die Kette des gauzen Muskels besteht aus den nebeneinander geschalteten Elementeu der einzelnen Fasern, vergleichbar einer Kette, deren positiven und negativen Pole miteinander leitend verbunden sind.

Fig. 5.

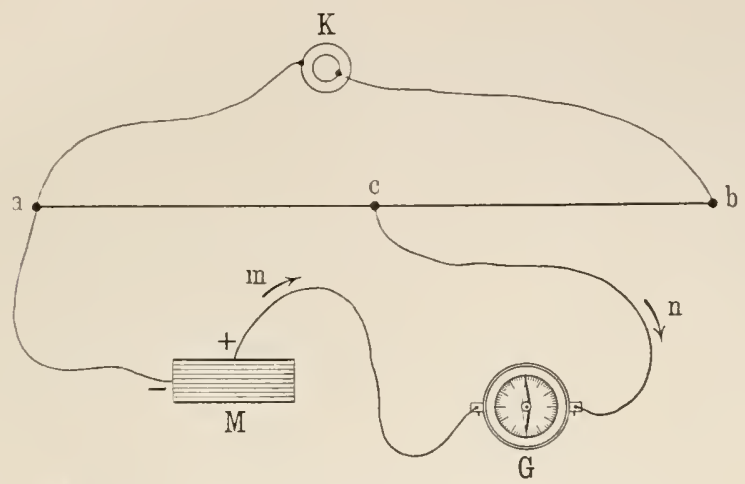

Messung der elektromotorischen Kraft durch Kompensation.

Da die einzelnen Fasern und Faserbündel von leitenden indifferenten Geweben (Bindegeweben und Gefäßen) mehr oder weniger umbüllt sind, so müssen nach Anlegung des Querschnittes innere Ströme entstehen, deren Intensität wegen des geringen Widerstandes in den kurzen Strecken un mittelbar am Querschnitt größer sein muß, als die des abgeleiteten Stromes in dem angelegten Galvanometerkreis. Daraus folgt, daß die wirkliche Potentialdifferenz zwischen Längs- und Querschnitt größer sein muß als die gemessene.

Die elektromotorische Kraft des abgeleiteten Stromes mißt man mit Hilfe der bekannten Poggendorff-du Bois-Re y mondschen Kompensationsmethode, indem man von einer bekannten Kette durch einen Rheochordwiderstand einen Zweigstrom von gleicher Stärke abnimmt und ihn in entgegengesetzter Richtung durch Muskel und Galvanometer leitet, wie es Fig. 5 zeigt. Von der 
Kette $K$ geht die Hauptleitung durch den Rheochorddraht $a b$ und ein Nebenstrom von den Punkten $a$ und dem Schleifkontakt $c$ durch das Galvanometer $g$ in den Muskel $M$. Hat der Muskelstrom von der Richtung des Pfeiles $m$ dieselbe Kraft, wie der Kompensationsstrom von der entgegengesetzten Richtung $p$, so steht das Galvanometer auf Null. Die Potentialdifferenz $e$ des Muskels ist gleich der der Punkte $a$ und $c$. Ist der Widerstaud der Leitung $K a b K=W$, der von $a c=w$, die Kraft von $K=E$, so ist

$$
e=E \cdot \frac{w}{W}
$$

Man kann ferner sich zur Bestimnung der Potentialdifferenzen auch zweckmäßig eines Elektrometers bedienen, eines Thomsonschen Quadrantelektrometers oder eines Kapillarelektrometers von hinreichender Empfindlichkeit.

Der unverletzte Muskel, von einem eben getöteten Tiere sorgfältig mit seinen beiden Endsehnen präpariert, zeigt entweder nur schwache Ströme oder ist unter gewissen Umständen fast stromlos. du Bois-Reymond nannte die Sehne des Muskels seinen natürlichen Querschnitt, da hier die Substanz der Fasern direkt mit Sehnenfasern verbunden ist. Man findet, daß, wenn der nuverletzte Muskel einen Strom besitzt, dann regelmäßig die Sehne sich negativ gegen den Längsschnitt verhält, aber dieser Strom ist bei weitem schwächer als der vom künstlichen Querschnitt abgeleitete. Wenn man die Sehne fortschneidet oder dieselbe vollständig durch Ätzung oder Hitze abtötet, so kommt der stärkere Längsquerschnittstroı zum Vorschein. Läßt man den unverletzten Muskel längere Zeit liegen, so verstärkt sich allmählich der Längsschnittsehnenstrom. du Bois-Reymond beobachtete, daß unversehrte Muskeln eines längere Zeit auf $0^{0}$ abgekühlten Frosches ganz stromlos sind. L. Hermann wies nach, daß auch mit größter Schonung vom lebenden Tier entnommene Muskeln nahezu stromlos erscheinen, wenn man sie von Längsschnitt und Sehne ableitet. Es ergibt sich hieraus, daß die unverletzte Muskelfaser, wenn sie sich in ihrer ganzen Ausdehnung bis zum Sehnenende in gleichem physiologischen Zustande befindet, an allen Punkten gleiches elektrisches Potential besitzt, also vach außen stromlos erscheinen muß. Sobald sich aber der physio- 
logische Zustand an irgend einer Stelle ändert, so entwickeln sich Potentialdifferenzen. Es erklärt sich daraus, daß der Muskel eines stark abgekühlten Tieres regelmäßig stromlos ist, weil bei der niederen Temperatur der Stoffwechsel aller Organe stark herabgesetzt wird und infolgedessen im Muskel der physiologische Zu1stand sich im gauzen Organ fast vollständig ausgleicht. Im normal ernährten Tiere bei höherer Temperatur ist aber eine solche Gleichheit des Zustandes fast niemals vollständig in allen Teilen eines Muskels vorhanden, und daher sehen wir fast regelmäßig schwache Potentialdifferenzen auch beim lebenden Tiere nach Fortnahme der Haut') und Ableitung der Muskeln von Längsoberfläche und Sehnenenden auftreten, oder auch an anderen Punkten. Da man diese schwachen Ströme eines oft unregelmäßigen und unverletzten Muskels, die häufig auf zufälligen geringen Läsionen, wie Quetschung, Druck usw. beruhen können, bei weiteren Fragen vernachlässigen kann, so wollen wir uns die unverletzte Muskelfaser im idealen Zustande einer gleichförmigen Beschaffenheit in ihrer ganzen Ausdehnung als stromlos vorstellen, wie das aus den Untersuchungen von L. Hermann zu folgern ist. Die Frage nach der Ursache der Läsionsströme wollen wir vorläufig noch unentschieden lassen. Man kann indessen die Bedingung ihres Anftretens in folgender Weise formulieren: Sobald a uf irgend eine Weise durch einen indifferenten Leiter das Innere einer Faser mit der Oberfläche derselben in Verbindung gesetzt wird, so entsteht ein Strom in angegebener Richtung. Dieser indifferente Leiter kann aus abgetöteter Muskelsubstanz, Sehnensubstanz, Gewebsflüssigkeit und Elektrodenflüssigkeit (physiologische Kochsalzlösung) usw. bestehen.

L. Hermann formulierte diese Bedingung, indem er sagte, daf der Längsquerschnittstrom durch den Konfakt toter bzw. absterbender und lebender Muskelsubstanz entstehe. Indessen in dieser Formulierung liegt schon eine theoretische Behauptung, nämlich die, daß das Kontaktpotential zwischen lebender und toter bzw. absterbender Muskelsubstanz die Ursache des Stromes sei. Die weitere Erörterung dieser Fragen wollen wir in einem späteren Kapitel vornehmen.

^) Die Haut muß entfernt werden, da sie selbst elektromotorisch wirkt (s. weiter unten). 
Von Wichtigkeit ist noch die Feststellung, daß ein völlig abgetöteter M uskel stromlos ist, da $\mathrm{b}$ also die elektromotorische Eigenschaft der Muskeln zu den Lebenseigenschaften gehört. Ist der Muskel nach dem Tode unerregbar gewrorden und schließlich der Totenstarre verfallen, welche bekanntlich mit einer eingreifenden chemischen Veränderung verknüpft ist, so ist der Längsquerschnittstrom verschwunden. Dies tritt beim Erhitzen auf 45 bis $50^{\prime \prime}$ sehr schnell ein, beim zeitlichen spontanen Absterben langsam.

Fig. 6 .

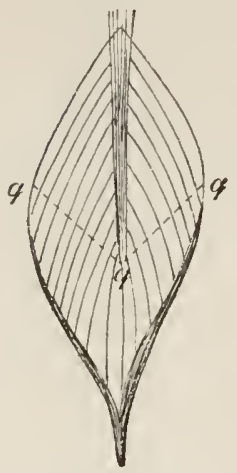

Bau des Musculus gastrocu. v. Frosch. qq physiologischer Querschnitt.

Mannigfache Abänderungen erleidet das Auftreten von Strömen au unregelmäbig gestalteten Muskeln, z. B. am Musculus gastrocnemins (Wadenmuskel) des Frosches, welcher in Fig. 6 im frontalen Längsschnitt dargestellt ist. Man sieht, daß sich eine obere platte Sehne in das Innere des Muskelbauches tief einsenkt, vou der die MIsskelfasern doppelt federförmig ent. springen, und daß über die untere Hälfte des IInskels die Achillessehne sich in einer sehnigeu Haut ausbreitet, an welcher sich die Muskelfaser'n ansetzen. Eine durch alle Muskelfasern senkrecht gelegte Fläche gibt den sogenannten physiologischen Querschnitt $q q q$ an, welchem bekanntlich die Muskelkraft proportional ist. In der oberen Hälfte des Muskels liegt nur der Längsschnitt der Fasern frei, in der unteren dagegen hauptsächlich Sehnenenden derselben als sogenanuter natürlicher Querschnitt. Leitet man daher von der Achillessehnenausbreitung und der oberen Hälfte des Muskels ab, so erhält man häufig einen merklichen, im Muskel von unten nach oben fließenden Strom, namentlich in der wärmeren Jahreszeit. Der obere natürliche Querschuitt kommt wegen seiner Lage im Inneren nicht zur Wirkung. Da eine große Zahl von Muskeln der unteren Extremitäten ähnlichen Bau haben, so erklärt sich daraus der von Nobili beobachtete, von den Zehen nach der Wirbelsänle gerichtete Froschstrom. Es erklärt sich daraus die Galvanische Zuckung ohne Metalle, welche auftritt, wenn man den Nerv. ischiadicus auf die Rü ckseite des Wadenmuskels fallen läßt, da in diesem Moment der Strom desselben durch den Nerven fliebt, wenn er Bauch und Sehnenspiegel berïhrt. In derselben Weise erklären sich 
die von Alex. v. Humboldt beobachteten Zuckungen bei Berührung eines Schenkelnervs mit Längsschnitt und künstlichem Querschnitt eines anderen Muskels. Auch zwei Punkte des Sehnenspiegels geben meist einen aufsteigenden Strom im M. gastrocn., weil an einem schrägen künstlichen Querschnitt Potentialdifferenzen auftreten, die sich durch die treppenförmige Aneinanderlagerung der Fasern am Querschnitt erklären. Es hat kein besonderes Interesse, die Erscheinungen der Stromentstehung an verschiedenen unregelmäßig gebauten IIuskeln zu verfolgen. Innerhalb des lebenden Körpers unter normalen Bedingungen der Ernährung der Muskelu treten derartige Ströme, solange der Zustand in allen Fasern in ibrer ganzen Ausdehnung als gleichförmig angesehen werden kann, überhaupt nicht auf.

Von hohem Interesse war es nun, zu untersuchen, wie sich der Muskelstrom bei der Tätigkeit des Muskels verhält. Man kann den isolierten lebenden Muskel bekanntlich künstlich reizen, am besten, indem man durch ihn oder durch seinen Nerven elektrische Ströme, entweder Induktionsströme oder unterbrochene konstante Ströme in schneller Folge hindurchleitet. Es entsteht dam eine anhaltende Kontraktion des Muskels, welche man Tetanus (Krampf) nennt. du Bois-Reymond entdeckte, daß der Muskelstrom während des Tetanus abnimmt. Er nannte diesen Vorgang "die negative Schwankung" des Muskelstromes. Nit empfindlichen Galvanometern konnte man auch bei einem einzelnen Reizstrom eine schwache negative Schwankung nachweisen von offenbar sehr kurzer Dauer, woraus folgte, daß die Gesamtschwankung im Tetanus sich aus einer mit den Reizen isochronen Reihe von Einzelschwankungen zusammensetzt, wobei der Strom in schneller Folge ab- und aufsteigt. In Fig. 7 sieht man die Anordnung eines solchen Versuches am Musc. gastrocn. und Nerv. ischiad. des Frosches. Der Muskel $m$ wird von seiner Rückseite mit den Elektroden $r$ zum Galvanometer $G$ abgeleitet. Zeigt der Muskel keinen merklichen Strom, so tötet man die Sehnenschicht (durch Ätzung mit verdünnter Karbolsäure, Alkohol oder mit heißem Stab) ab. Nan beobachtet nun die Ablenkung durch den Strom des ruhenden Muskels — "Ruhestrom". Vorher ist der Nerv $n$ auf die Elektroden der sekundären Spule $S$ eines Schlitteninduktoriums gelegt, durch dessen primäre Spule $P$ mit Hilfe des Wagnerschen Hammers die unterbrochenen Ströme 
eines Elementes $B$ geleitet werden. Ist der als Kurzschluß dienende Schlüssel $S$ geschlosseu, so gehen die Induktionsströme nicht durch den Nerven. Öffuet man den Schlüssel, wie Fig. 7 zeigt, so sieht ınan, daß gleichzeitig mit der tetanischen Kontraktion des Muskels die Ablenkung vom Galvanometer erheblich abnimmt und während des ganzen Tetanus vermindert bleibt. Erst mit der Ermüdung des Muskels nimmt gleichmäßig Stärke der Kontraktion und Größe der negativen Schwankung ab. Wiederholt man den Versuch mit einzelnen Ruhepausen, so beobachtet man, daß der Muskel sich Fig. 7.

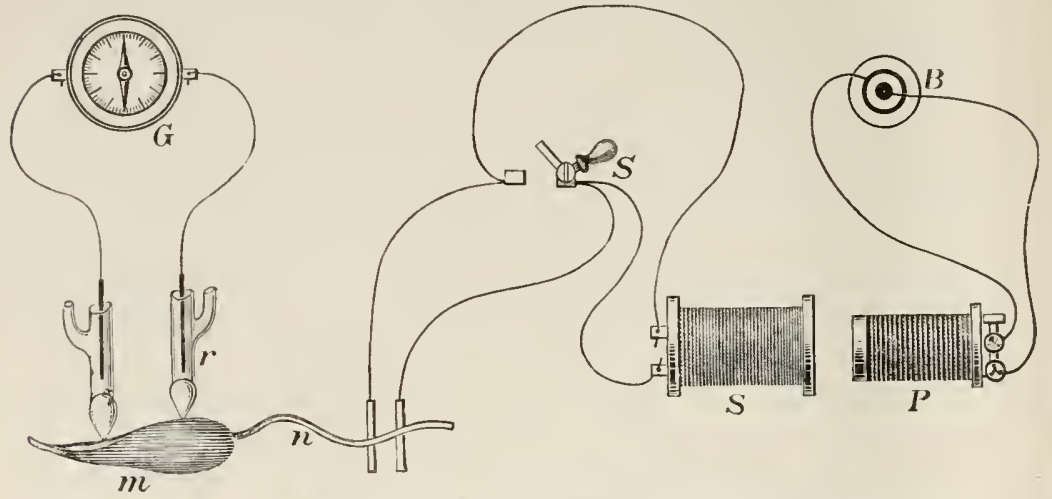

Negative Schwankung des Muskelstromes.

$m$ Muscul, gastrocnemius von Sehne und Längsschnitt abgeleitet, $n$ Nerv, $r r$ umpolarisierbare Elektroden, $G$ Galvanometer, $S$ schlussel, $B$ Batterie, $P$ primäre, $S$ sekundäre Spule.

durch Ruhe wieder erholt, sowohl betreffs der Kontraktion, wie der negativen Schwankung, aber durch schnelle Wiederholung der Reizung macht sich an beiden Prozessen sehr bald die Ermüdung bemerkbar, und man sieht bei vorher stark entwickeltem Ruhestrom, daß derselbe allmählich abnimmt, schneller als durch zeitliches Absterben in der Ruhe. Auch bei direkter Reizung des Muskels erhält man dasselbe Resultat, nur muß bei elektrischer Reizung verhütet werden, daß die Reizströme iu das Galvanometer gelangen. $\mathrm{Zu}$ diesem Zwecke bedient mau sich eines möglichst langen und dünnen Muskels, aus parallelen langen Fasern zusammengesetzt, z. B. des Musc. sartorius (Schneidermuskel), vom Oberschenkel des Frosches, reizt ihn an einem Ende und leitet voin anderen Ende den Längsquerschnittstrom ab. Dieser Muskel 
oder auch der Musc. adductor. longus am Oberschenkel ist wegen seines regelmäßigen Baues aus langeu durchgehenden parallelen Fasern hauptsächlich zu genaueren Untersuchungen benutzt worden.

Der Muskelstrom und die negative Schwankung desselben sind also mit der Lebenseigenschaft und Lebenstätigkeit eng verknüpfte elektrische Erscheinungen. lhre weitere Erforschung läßt daher erwarten, daß sie zu Aufschlüssen über den Zusammenhang der Lebensprozesse im Muskel und anderen Organen führen wird.

du Bois-Reymond hat bewiesen, dab die negative Schwankung nicht durch Formveränderung des Muskels bei der Kontraktion hervorgerufen wird; denn wenn man den Muskel durch Fixierung seiner Sehnenenden an der Verkürzung verhindert, so tritt sie ebenfalls auf. Die Verkürzung und Verdickung des Muskels bei ungehinderter Zusammenziehung müßte überdies wegen Verminderung des Leitungswiderstandes den entgegengesetzten Erfolg haben, wenn der spezifische Widerstand des Muskels derselbe bleibt. Nach dem Ohmschen Gesetz ist bekanntlich die Stromstärke gleich der elektromotorischen Kraft dividiert durch den Widerstand. Es könnte daher eine Verminderung des Stromes bei der Reizung durch Vermehrung des spezifischen Widerstandes herbeigeführt werden. Dies ist aber nicht der Fall. Die negative Schwankung wird vielmehr durch eine Abnahme der elektromotorischen liraft des Muskelstromes liervorgernfen. Dies zeigte du Bois-Reymond, indem er den Muskelstrom durch einen gleich ștarken entgegengesetzten Strom kompensierte und beobachtete, daß bei der Reizung der Ausschlag des Galvanometers im Sinne des Kompensationsstromes eintrat. Da beide Ströme denselben Widerstand haben, so folgt daraus, daß die Kraft des Muskelstromes sich vermindert.

Von du Bois-Reymond ist ferner nachgewiesen worden, daß die negative Schwankung im Tetanus in einer schnellen Folge kurzer Einzelschwankungen besteht. Dies läßt sich aus dem sekundären Tetanus schließen, den man beobachtet, wenn man deu Nerven eines Nervmuskelpräparates in bestimmter TVeise einem ander'en Muskel anlegt, wie es Fig. 8 zeigt. Letzter'er, der primäre Muskel, wird von seinem Nerven aus gereizt, und sobald el. sich kontrahiert, kontrahiert sich auch der sekundäre Muskel. 
Diese Erscheimung ist erst von du Bois-Reymond richtig gedeutet worden. Der sekundäre Nerv muß dem primären Muskel so anliegen, daß er denselben möglichst in der Längsrichtung der Fasern, am besten den Längsschnitt und die Sehne oder beim verletzten Muskel den Längsschuitt und Querschnitt berülırt. In diesem Falle bildet er einen Leitungsweg für Schleifen der Riuheströme und der entstehenden Stromesschwankungen, und da bekanutlich der Nerv hauptsächlich anf Stromesschwankungen reagiert, so wird er dadurch gereizt. Schon eine einzelne primäre Zuckung hat eine sekundäre zur Folge, wie zuerst Matteucci beobachtete. du Bois-Reymoud zeigte aber, daß auch ein sekundärer Tetanus auftritt, der so lange anhält wie der primäre, und daß es Stromesschwankungen sind, die ihn hervorrufen. Legt man den sekundären Nerven quer über die obere oder unter'e 2. Sehue des primären Muskels, so bleiben die sekundären KontrakFig. 8.

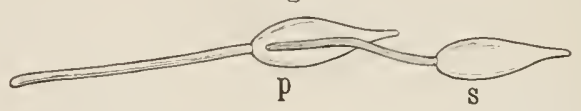

sekundäre Zuckung und sekundärer Tetanus.

tionen aus, ein Beweis dafür, daß nicht etwa Reizströme, aus dem primären Nerven nach dem sekundären hingeleitet, die Ursache der Erscheinung sind. Auch der quer über den Muskel gelegte Nerv reagiert nicht, oder in gewissen Fällen nur schwach, was sich aus unregelmäßigem Bau des Muskels oder ungleichförmiger Aktion seiner Fasern erklären läßt.

Ebenso wie die negative Schwankung auch bei nicht elektrischer, bei mechanischer oder chemischer Reizung auftritt, so lassen sich auch bei dieser Reizungsart sekundäre Wirkungen beobachten. Zum Zwecke der rhythmischen mechanischen Reizung des Nerven hat Heidenhain einen Tetanomotor konstruiert, welcher aus einem Elfenbeinhämmerchen besteht, der an eiuem Hebel befestigt ist, welcher nach Art des Wagnerschen Hammers in schnelle Schwingungen versetzt wird und der einen darunter gelagerten Nerven eines Muskels rhythmisch reizt, so daß Tetanus entsteht. Auch dieser hat negative Schwankung und sekundären Tetanus zur Folge. Weniger deutlich ist dies bei chemischer Reizung des Nerven, weil hierbei keine so deutliche Rhythmik auftritt. 
Ebenso wie am Muskel fand du Bois-Reymond auch am Nerven, wenn man den Längsschnitt desselben (natürliche Längsoberfläche) mit einem künstlichen Querschuitt verbindet, einen Strom vor, welcher dieselbe Richtung hat wie im Muskel. Jeder künstliche Querschnitt verhält sich negativ gegen den Längsschnitt, auch gegen einen künstlichen, durch Längsteilung hergestellten. Es folgt daraus, daß auch jeder einzelnen Nerrenfaser, aus denen die Nervenstämme zusammengesetzt sind, dieselbe Eigenschaft zukommt. Die Nervenfasern, welche bekanntlich zum größten Teile im Gehirn und Rückenmark aus Nervenzellen als Fortsätze derselben entspringen und in ihrer ganzen Länge die Körperteile kontinuierlich durchziehen, bis sie als motorische in den Muskeln oder als sensible und sensorische in den empfindenden Organen, namentlich der Haut und den Sinnesorganen, endigen, besitzen in ihrem Verlauf kein abgeschlossenes isolierbares natürliches Ende, wie die Muskelfasern an den Sehnen, also keinen sogenannten natürlichen Querschnitt ${ }^{1}$ ).

Die Kraft des Nervenstromes, welchen man zum Galvanometer ableitet, beträgt etwa $0,02 \mathrm{Volt}$, doch muß man annehmen, daß die inneren Ströme viel stärker sind, da die Nervenfaserbündel von ziemlich ansehnlichen Bindegewebshüllen umschlossen werden, durch die ein merklicher Teil des Längsquerschnittstromes abgeleitet wird. Den wahren Wert der Kraft des Nervenstromes kennt man daher nicht, während die des inneren Muskelstromes nach einer Schätzung von L. Hermann nicht viel größer ist als 0,08 Volt. An allen Nerven des Körpers der verschiedenartigsten Funktion ist das Gesetz des Nervenstromes dasselbe, und es ist dabei auch gleichgültig, ob man einen nach der Peripherie oder dem Zentrum hin gerichteten Querschnitt ableitet. Ein von zwei künstlichen Querschnitten begrenztes und aus gleich langen Fasern zusammengesetztes Stück eines Nerven verhält sich nach beiden Richtungen hin elektrisch gleichartig. Es zeigt ebenso wie der IIuskel auch schwache Ströme des Längsquerschnittes, indem ein

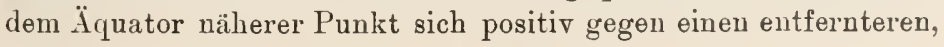

1) An der Peripherie verbinden sich die feinsten Nervenfasern in verschiedener Art mit anderen Gewebselementen, z. B. mit Sinneszellen. An der Netzhaut z. B. erscheinen auch Potentialdifferenzen nnd elektrische Prozesse (s. unten). Man könnte sie als einen natürlichen Querschnitt des Nervus opticus ansehen. 
dem Querschnitt näheren Punkt verhält. Der Strom verschwindet, wenn der Nerv auf irgend eine Weise abgetötet wird, sei es durch Hitze oder chemische Reagenzien, oder Zerquetschung; er gehört also zu den Lebenseigenschaften desselben. Beim spontanen Absterben des Nerven außerhalb des Körpers sinkt der Strom des Nerven allmählich herab. Indessen ist von Engelmann nachgewiesen worden, daß unter günstigen Bedingungen (Verhütung der Austrocknung in feuchter Kammer und nicht zu hohe Lufttemperatur) der Strom auf Null absinken kann, ohne daß der Nerv abgestorben ist. Ein frischer Querschnitt in der Nähe des alten stellt den Längsquerschnittstrom wieder her. Dies sieht man am Muskel niemals und ist nach Engelmann darauf zurückzuführen, daß die Fasern aller peripheren Nerven aus Teilstücken zusammengesetzt sind, welche der Histologe Ran vier zuerst demonstriert hat und die etwa $0,5 \mathrm{~mm}$ lang sind. Jhre Grenzen markieren sich durch eine Einschnürung des Nervenmarks und Neurolems, welche den Achsenzylinder der Faser umhüllen. Jedes Teilstück besitzt den Wert einer in ihrer Funktion zum Teil selbständigen Zelle, und solche der Länge nach aneinandergereihten Zellen, wie sie sie durch Wachstum bei der Entwickelung entstehen, bilden die ganze Nervenfaser. Ist ein solches Teilstück verletzt, so stirbt es iufolge dieses Eingriffes im Verlauf einiger Stunden ab. Aber (der Prozeß des Absterbens macht, unter günstigen Bedingungen, an dem nächsten Ranvierschen Schnürring zunächst Halt.) Alsdann verhält sich die Faser an diesem Schnürring wie eine daselbst natürlich geschlossene Zelle, welche immer stromlos ist. (Erst allmählich stirbt bei Mangel der Ernährung der Nerv in toto ab.) Ganz anders ist der Vorgang an einer verletzten Muskelfaser. Von der Verletzung aus breitet sich der Absterbeprozeß bis an das Ende der Faser aus, denn die ganze Faser ist als eine einzige langgestreckte Zelle anzusehen.) Der Strom sinkt allmählich ab, aber niemals verstärkt er sich wieder bei einer neuen Verletzung ${ }^{1}$ ). Wir werden auf diese wichtigen Tatsachen bei der Theorie der bioelektrischen Ströme nochmals

1) Nur wenn die Muskelfasern durch Sehnengewebe unterbrochen sind (Inscriptiones tendineae, z. B. an den Bauchmuskeln), macht an dieser Stelle der Absterbeprozeß Halt. Ein neuer Querschnitt daselbst ruft den gesunkenen Strom wieder hervor. Ähnlich ist es bei glatter Muskulatur und dem Herzmuskel (s. weiter unteu). 
zurückgreifen. Es sei noch hinzugefügt, daß Engelmann auch das elektrische Verhalten der verletzten Nerven und Muskeln im lebenden Körper untersucht hat. Unter günstigen Bedingungen der Ernährung tritt sehr bald auch am Muskel eine Abgrenzung unter Verschwinden des Längsquerschnittstromes ein. Diesen Vorgang, den man als Beginn des mit komplizierten Wachstumsprozessen verknüpften Heilungsvorganges anzusehen hat, kann man als "Demarkation" bezeichnen (s. oben). Die Demarkation nach der Verletzung ruft also nicht den Strom hervor, sondern im Gegenteil, sie bringt ihn zum Verschwinden.

Der Längsquerschnittstrom der Nervenzeigt ebenso wie der des Muskels bei jeder Art der Reizung eine negative Schwankung, die ebenfalls a uf einer Verminderung der elektromotorischen Kraft desselben beruht. Bei elektrischer Reizung muß dafür gesorgt sein, daß die Reizströme nicht zum Galvanometer durch Stromschleifen gelangen. Man leitet einen möglichst langen Nerven an einem Ende vom Längsund Querschnitt ab und legt ihn am anderen Ende über die Elektroden des Reizstromes. Am besten verwendet man hierzu wiederum die Wechselst röme der sekundären Spule eines Schlitteninduktoriums von mäßiger Stärke. Da diese in ihrer Wirkung anf ein Galvanometer sich aufheben, so können sie nicht so leicht zu täuschenden Ablenkungen Veranlassung geben. Man überzeugt sich davon, wenn inan den Nerven an einer Stelle zwischen den ableitenden und erregenden Elektroden durchschneidet und wieder aneinanderlegt, oder ihn mit feuchtem Faden unterbindet oder auch völlig durchquetscht. Hierdurch wird jede Leitung der Erregung aufgehoben. Dasselbe Verfahren muß man auch bei direkter Muskelerregung (s. oben) anwenden, wo es wegen des größeren Querschnittes leichter zu Stromschleifen kommen kann, als bei dem verhältnismäßig dünneren Nerven. Auch unterbrochene konstante Ströme lassen sich zur Reizung benutzen, doch tut man in diesem Falle gut, Wechselströme zu wählen, da Ströme von konstanter Richtung noch eine andere Änderung infolge der inneren Polarisation hervorrufen, die bei der einen Richtung die negative Schwankung verstärken, bei der anderen dagegen schwächen und dadurch den Sinn der Ablenkung umkehren können (s. weiter unten). Auch bei tetanischer Reizung mit Induktionsströmen ist es geraten, sich bei genaueren Messungen nicht des gewöhnlichen 
Wagnerschen Hammers zu bedienen, sondern der Helmholtzschen Modifikation desselben, welche bewirkt, daß Öffnungs- und Schliebungsinduktionsströme annähernd gleiche Dauer und Intensität annehmen ${ }^{1}$ ). Auch auf einzelne Induktionsschläge reagiert der Nervenstrom mit einer an empfindlichen Instrumenten wahrnehmbaren negativen Schwankung (s. unten). Die mechanische und chemische Reizung bewirken, wenn auch viel schwächere, aber doch deutliche Schwankungen in derselben Richtung. Man erkennt also daraus, daß der Zustand der 'lätigkeit des Nerven, den man an motorischen Nerven durch Muskelkontraktion, an den seusibeln Nerven im lebenden Körper durch Empfindungen wahrnimmt, am isolierten Nerven immer mit einer negativen Schwankung des Nervenstromes verknüpft ist. Da sie bei jeder Art der Erregung eintritt, ist sie ein Zeichen des Erregungsprozesses. Man kann daher durch dieselbe auch die Fortleitung des Erregungsprozesses in den Nerven erkennen. Von diesem Gesichtspunkte ausgehend hat du Bois-Reymond, dem wir die Kenntnis der bisher angeführten Tatsachen verdanken, auch untersucht, wie sich die Nerven verschiedener Funktion darin verhalten und nach welchen Pichtungen sie den Erregungsprozeß leiten. Man hat lange geglaubt, daß die motorischen Nerven, welche während des Lebens immer nur von den Nervenzentren zu den Muskeln Impulse leiten, auch nur in dieser zentrifugalen Richtung leiten könnten, und daß umgekehrt alle sensibeln Siunesnerven, welche während des Lebens von den empfindlichen Organen und den Sinnesorganen zu den Zentren leiten, auch nur in zentripetaler Richtung leiten könnten. Zum ersten Male zeigte du BoisReymoud durch das Experiment, daß dem nicht so sei. Er fand an rein motorischen Nerven, daß, wenn man das zentrale Ende derselben zum Galvanometer ableitet und ihr peripheres Ende reizt, sie ebenso gut wie bei umgekehrter Anordnung negative Schwankung geben, und auch, wenn man rein sensible Nerven am zentralen Ende reizt und das periphere Ende mit dem Galvanometer verbindet, dieselbe Erscheinung eintritt. Solche Versuche lassen sich gut mit den vorderen und hinteren Wurzeln der Rücken-

1) Bekanntlich hat der Öffnungsinduktionsschlag einer sekundären Spule eine größere Intensität, aber geringere Dauer als der Schließungsinduktionsşchlag. 
marksnerven anstellen, von denen die ersteren nur motorische, die letzteren nur sensible Fasern enthalten, ferner a n Nerv. opticus (Sehnerv) und Nerv. olfactorius (Geruchsnerv), welche reine Sinnesnerven sind, und am Nerv. oculomotorius (Augenmuskelnerv), welcher rein motorisch ist. Die Nerven besitzen also ein doppelsinniges Leitungsvermögen. Daß sie während des Lebens nur in einem Sinne leiten, entweder zentrifugal oder zentripetal, hat seinen Grund nur darin, daß die ersteren nur im Gehirn und Rückenmark, die letzteren nur in den peripheren Organen ihre Reize aufnehmen. Ob die Nervenfasern verschiedener Funktion in jeder Beziehung einander gleichwertig sind, wollen wr an diesem Orte nicht weiter verfolgen. Jedenfalls unterscheiden sie sich auch histologisch und chemisch nicht wesentlich voneinander, und in ihren bisher bekannten physikalisch-physiologischen Eigenschaften zeigen sie keine Verschiedeuheiten. Der Erregungsproze $\$$ in ihnen wird daher, wie man hieraus schliefen kann, nicht wesentlich verschiedener Natur sein.

Die elektrischen Veränderungen bei der Reizung der von allen Organen isolierten Nerven sind bis jetzt die einzigen Erkennungszeichen der Tätigkeit. Man hat ihre Fnnktion verglichen mit der Rolle, welche die Telegraphendrähte spielen, und sich volgestellt, daß sie wie diese Nachrichten vom Gehirn zu Organen und von diesen zum Gehirn führten. Indessen ist dieser Vergleich unhaltbar, und es muß ausdrücklich hervorgehoben werden, daß beide Vorgänge total verschiedener Natur sind. (Die elektrischen Vorgänge in den Nerven bestehen nicht, wie in den Telegraphendrähten, in der Fortleitung eines elektrischen Stromes nach der einen oder andeı en Richtung, sondern beruhen anf einer physiologischen Zustandsänderung, welche, mit chemischen und physikalischen Prozessen verknüpft, sich von einem Ende zum anderen fortpflanzt.) Helmholtz hat ermittelt, daß die Geschwindigkeit der Reizleitung in motorischen Froschnerven etwa 26 bis $30 \mathrm{~m}$ in der Sekunde beträgt, während die Geschwindigkeit der Elektrizität gleich der Lichtgeschwindigkeit zu setzen ist. Zur Leitung eines Stromes bedürfte es auch einer Rückleitung, während der vom Körper isolierte Nerv allein physiologisch leitet. In früheren Zeiten der Naturphilosophie glaubte man, daß die Nerven mit Lebenskräften besonderer Art begabt seien, und daß es ein geheimnisvolles Fluidum gäbe, welches in ihnen anf - und ab- 
fließe ${ }^{1}$ ). Die Untersuchungen ron Helmholtz und du BoisReymond haben hauptsächlich dazu beigetragen, diesen Aberglauben zu zerstören.

du Bois-Reymond stellte zur Erklärung der Muskel- und Nervenströme und ihrer Veränderungen bei der Reizung die Hypothese auf, daß die Muskel- und Nervenfasern elektromotorische Molekeln enthalten, welche er als Sinnbild der Verteilung elektrischer Spannungen in den kleiusten Teilchen der lebenden Substanz der Fasern betrachtete. Er dachte sie sich in der Längsrichtung der Fasern in Reihen so angeordnet, daß sie ibre positiven Spannungen dem Längsschnitt, ihre negativen den Querschnitten zuwenden. Diese Hypothese setzte also eine Präexistenz der elektrischen Potentiale in kleinsten Elementen der Fasern voraus. Er deutete hiernach das Vorhandensein eines Längsquerschnittstromes und auch das häufige Auftreten von Längsschnittsehnenströmen. Die negative Schwankung erklärte er durch Abnahme dieser elektromotorischen líräfte bei der Tätigkeit, wobei man an einen Verbrauch derselben denken konnte.

Dieser Präexistenztheorie gegenüber stellte L. Hermann im Jahre 1867 eine sogenannte Alterationstheorie auf. Das Auftreten eines Längsquerschnittstromes deutete er durch Entstehung eines Kontaktpotentials zwischen der abgestorbenen oder absterbenden Substanz der Faser am künstlichen Querschnitt und der lebenden Substanz am Längsschnitt. Wir wollen auf die so entstandene Streitfrage zwischen Präexistenz und Alteration an dieser Stelle noch nicht eingehen, sondern, wie schon oben bemerkt, nur die Bedingung für die Entstehung eines Längsquerschnittstromes dahin formulieren, daß hierzu die Bloßlegung des Faserinhaltes durch eine Verletzung erforderlich ist.

$\left.{ }^{1}\right)$ Die in neuerer Zeit von v. Üxkïll geäußerte Anschauung, nach welcher der Vorgang im Nerven wiederum mit dem Fließen eines Fluidums in Röhren verglichen wurde, entbehrt jeder tatsächlichen Grundlage und erscheint mir nicht einmal als Arbeitshypothese zulässig. 


\section{Zweites Kapitel. \\ Theorie der elektrischen Ketren.}

Von großer Bedeutung für das Verständnis der elektrischen Ketten wurden die hervorragenden theoretischen Studien von W.Gibbs und v. Helm holtz auf dem Gebiete der Thermodynamik in den letzten Jahrzehnten des vorigen Jahrhunderts. Hierzu gesellten sich die grundlegenden experimentellen Forschungen von v. Helmboltz ïber die Entstehung von Kionzentrationsströmen und die bald darauf folgenden Untersuchungen von Nernst über die Kräfte der Konzentrationsketten im Lichte der neueren Ionentheorie von Arrhenius und Ostwald.

Diese ausgezeichneten Forschungen mußten sehr bald eine Einwirkung auf die weitere Entwickelung der bioelektrischen Untersuchungen ausüben. Bevor wir auf dieses Thema näher eingehen, sollen die wesentlichsten physikalischen Grundlagen, welche zum Verständnis desselben liotwendig sind, zınächst gegeben 'werden.

W. (Gibbs ${ }^{1}$ ) und v. Helmholtz ${ }^{2}$ ) hatten unabhängig voneinander ein wichtiges Prinzip der Energielehre von der allgemeinsten Bedeutung für alle Naturvorgänge aus den beiden Hauptsätzen der mechanischen Wärmetheorie mathematisch hergeleitet. Dieses gilt für alle umkehrbaren, isothermen Prozesse, d. b. für solche, bei denen eine Energieumwandlung auch in der umgekehrten Richtung ohne einen Verlust an Energie stattfinden kann, und bei denen die Temperatur, sei es durch Wärmezufuhr oder-abfuhr, konstant erhalten wird. Ein solcher Vorgang ist bekanntlich im Carnotschen Kreisprozeß enthalten, bei welchem Wärme in Zylinder einer Dampfmaschine in Arbeit verwandelt wird. Dieser Prozeß kann auch umgekehrt ausgeführt werden,

1) Transactions of the Connecticut Academy III, 1876-1878 (s. Ostwald, Elektrochemie, S. 992), und Thermodynamische Studien. Dentsch von Ostwald 1892.

2) Zur Thermodynamik chemischer Vorgänge. Ges. Abhandl. •”, 979 (1882). 
indem man die geleistete Arbeit dazu verwendet, um den Dampf im Zylinder zu komprimieren und wieder in den anfänglichen Zustand zurückzuführen. Hierbei wird gerade soviel Wärmeenergie wieder gewonnen, als zur Arbeitsleistung verbraucht war. Bekannt ist es nun, daß bei einem solchen thermischen Kreisprozeß nicht die ganze dem Dampf zugeleitete Wärme in mechanische Arbeit verwandelt werden kann, sondern nur ein bestimmter Bruchteil derselben, den man bei einer Maschine den „ökonomischen Koeffizienten" genannt hat und der von den absoluten Temperaturen abhängt, bei denen die Umwandlungen geschehen. Ist $Q_{1}$ die dem Dampf des Zylinders während der Arbeitsleistung zugeführte Wärmemenge und $Q_{2}$ diejenige, welche er nach geleisteter Arbeit an Ende des Ḱreisprozesses, d. h. wenn der Kolben wieder in die anfängliche Lage zurückgekehrt ist, besitzt, so ist $Q_{1}-Q_{2}$ die in Arbeit verwandelte Wärmemenge und $\frac{Q_{1}-Q_{2}}{Q_{1}}$ der ökonomische Koeffizient. Beim Heben des Kolbens, während der Leistung ïußerer Arbeit, muß die absolute höhere 'Temperatur $T_{1}$ konstant erhalten werden, und nach gespeicherter Arbeit beim Herabgehen des Kolbens in die Anfangslage muß die niedere Temperatur $T_{2}$ konstant erhalten werden. Dann ist nach einem bekannten Satze von Clausius der ökonomische Koeffizient:

$$
K=\frac{Q_{1}-Q_{2}}{Q_{1}}=\frac{T_{1}-T_{2}}{T_{1}} .
$$

Dieser Teil der in Arbeit verwandelbaren Wärmeenergie ist von Helmboltz als "freie Energie" bezeichuet worden gegenüber' der Gesamtenergie des Systems, welche bei dem stattfindenden Prozeß in Aktion tritt. Mau kann sich zur Erklärung dieses Verhaltens vom Standpunkte der kinetischen Gastheorie aus die Vorstellung machen, daß die Wärmeenergie in einer "ungeordneten "Bewegung der Moleküle und Atome bestehe, d. b. daß die Bewegungen und Schwingungen derselben nach allen möglichen Dimensionen des Raumes exfolgen, daß aber zur Erzeugung mechanischer Arbeit nur in bestimmten Richtungen geordnete $\mathrm{Be}$ wegungen verwendbar sind, wie z. B. zur Hebung von Lasten gegen die Schwere. Dieser Begriff der freien Energie läßt sich aber auch auf andere Energieformen übertragen, so daß man bei einer Energieumwandlung in einem System von Körpern die freie 
Energie, welche sich in Arbeit umsetzen kaun, von der gesamten in Aktion tretenden Energie des Systems unterscheiden kann. Es ergibt nun die weitere Betrachtung, daß die freie Energie eines Systems sich mit der absoluten Temperatur ändern muß, und diese Änderung neunt man den Temperaturkoeffizienten einer bestimmten freien Energieform. Derselbe kann mit steigender Temperatur zunehmen oder abnehmen, und es hat hiernach die in Betracht kommende Energieform einen positiven oder negativen Temperaturkoeffizienten.

Nennt man die Gesamtenergie eines Systems, welche in einem umkebrbaren isothermen Prozeß in Aktion tritt, $U$, und die hierbei auftretende freie Energie $F$, die absolute Temperatur, bei welcher der Prozeß erfolgt, $T$, so ist der Bruch $\frac{d F}{d T}$ der Temperaturkoeffizient der betreffenden Energieform, und die mathematische Behandlung des Problems ergibt die von Helmboltz entwickelte Formel:

$$
F=U \pm T \cdot \frac{d F}{d T}
$$

Es läßt sich das in dieser Formel gegebene Prinzip folgendermaßen in Worten ausdrücken:

"Wenn in einem System durch einen umkehrbaren isothermen Prozeß eine Umwandlung von Energie stattfindet, so ist die freie Energie gleich der gesamten in Aktion tretenden Energie des Systems plus oder minus einer Größe, welche gleich dem Produkt aus der absoluten Temperatur und dem positiven oder negativen Temperaturkoeffizienten der freien Energie bei dieser Temperatur."

Da dieser Temperaturkoeffizient ein positiver oder negativer sein kann, so entsteht im letzteren Falle die freie Energie aus einem Teil der Gesamtenergie, im ersteren Falle dagegen, in welchem die freie Energie größer ist als die in Aktion tretende Energie des Systems, muß Energie von außen zugeführt werden, z. B. in Form von Wärme. Es kann daher auch der einfache Fall eintreten, daß der Temperaturkoefizient der freien Energie Null ist; dann verwandelt sich die gesamte Energie des Systems in freie Energie. Das letztere tritt z. B. ein bei der Bewegung 
der Himmelskörper gegeneinander oder beim schwingenden Pendel (abgesehen von der Reibung), da der Temperaturkoeffizient der Gravitationsenergie als Null angenommen werden kann.

Es kommt nun für unsere Zwecke darauf an, dieses Prinzip auf die elektrischen Ketten anzuwenden. Die elektrische Energie, welche dieselben durch den Strom erzeugen und welche in einem Kreise von möglichst geringem Widerstande, abgesehen von der geringen Temperaturerhöhung der Leitungen, durch eine elektrodynamische Maschine fast vollständig in mechanische Arbeit umgesetzt werden könnte, kann in diesem theoretisch gedachten Falle gleich der freien Energie gesetzt werden, welche in dem System der zu einem Kreise geschlossenen Kette entstehen würde. Die so erzeugte elektrische Energie, welche in der Zeiteinheit entsteht, ist nach bekannten Gesetzen der Elektrizitätslehre gleich dem Produkt von elektromotorischer Kraft und Stromstärke zu setzen, und wählen wir als Stromstärke die Einbeit derselben im elektromagnetischen MaGe aus, so können wir statt der elektrischen Energie auch die elektromotorische Kraft in die gegebene Formel einsetzen und wollen dieselbe mit $E$ bezeichnen. Nennen wir nun ferner die in der Zeiteinheit in Aktion tretende Gesamtenergie der Kette $U$, so können wir unter gewissen Bedingungen für alle Ketten die Formel aufstellen:

$$
E=U+T \cdot \frac{d E}{d T}
$$

Diese Bedingung besteht darin, daß der Prozeß ein unkebrbarer ist und isotherm abläuft.

Bei der Anwendung dieser Formel müssen die Werte von $E$ und $U$ in Maßeinheiten derselben Energieform ausgedrückt sein, z. P. in Wärmeeinheiten, in mechanischen Maßen oder am besten in elektrischen Maßen [Joule ${ }^{\mathfrak{l}}$ ) $=\mathrm{J}$ ].

Die Ketten, welche im folgenden in Betracht zu ziehen sind, sind die bekaunten Galvanischen, oder richtiger Voltaschen, und die Konzentrationsketten. In den ersteren tritt bekanntlich chemische Energie in Aktion, in den letzteren fehlt diese Energieform, insofer'n in ihnen keine chemischen Veränderungen, sondern nur

1) $1 \mathrm{~J}=239,1 \mathrm{cal}=10^{10} \mathrm{Erg}, \mathrm{s}$. Ostwald, Grundriß der allgemeinen Chemie 1899, S. 88 . 
Konzentrationsänderungen der schon darin enthaltenen chemischen Körper stattfinden.

Mit Berechnung der Kraft Voltascher Elemente aus der Wärmetönung des chemischen Prozesses hatte man sich schon lange beschäftigt. Für das Daniellsche Element, in welchem sich beim Stromdurchgang an der Zinkelektrode Zink auflöst unter Bildung von $\mathrm{ZnSO}_{4}$, und an der Kupferelektrode Kupfer aus $\mathrm{CuSO}_{4}$ niederschlägt, stimmte der Wert für die in der Zeiteinheit erzeugte elektrische Energie mit der verbrauchten chemischen Energie recht gut überein, und man erhielt demnach einen annähernd richtigen Wert für die elektromotorische Kraft derselben, wie zuerst $\mathrm{W}$. Thomson fand. Indessen bestätigte sich diese Berechuung bei anderen Ketten nicht.

Nach obiger Formel (2) haben wir nun bei Volt a schen Ketten die in Aktion tretende chemische Energie mit der Größe $U$ zu bezeichnen.

Der Temperaturkoeffizient derselben, $\frac{d E}{d T}$, ist der Messung zugänglich. Man findet, daß derselbe in Ketten verschiedener Zusammensetzung positiv oder negativ sein kann. Ketten, deren Kraft mit der steigenden Temperatur abnimmt, erwärmen sich bei Stromerzeugung; Ketten dagegen, deren Kraft sich mit steigender. Temperatur erhöht, kühlen sich bei der Stromerzeugung ab. Die ersteren kann man exotherme, die letzteren endotherme Voltasche Ketten nennen. Eine Kette, deren Temperaturkoeffizient nahezu Null ist, ist nun die Daniellsche, und daraus erklärt es sich, daß für dieselbe die Formel (2) übergeht in $E=L$, d. h. daß die elektrische Energie derselben durch die chemische Energie des Prozesses nahezu vollständig gedeckt wird. In Ketten dagegen mit negativem Temperaturkoeffizienten entsteht durch den chemischen Proze@ bei der Strombildung mehr Wärme, als sich in elektrische Energie umsetzt, sie erwärmen sich daher. $\mathrm{Zu}$ diesel gehören eine Anzahl bekannter Elemente, wie das Grovesche, Bunsensche u. a. ${ }^{1}$ ), welche demnach exotherme Ketten sind.

1) Das Daniellsche Element, mit CuSO, und $\mathrm{ZnSO}_{4}$ gefüllt, ist als vollkommen umkehrbar anzuseben, . h. wenn man den von ihm erzeugten Strom in ungekehrter Richtung durchleitet, so wird es durch dieselbe zugeleitete Elektrizitätsmenge wieder in den anfänglichen Zustand zurückgebracht. Dies gilt für andere gebräuchliche Elemente nicht. 
Sebr merkwürdig ist es nun, daß sich auch Voltasche Ketten konstruieren lassen, welche sich bei der Strombildung abkühlen. Es sind von Jahn ${ }^{1}$ ) über die Thermodynamik der Voltaschen Ketten eingehende Untersuchungen angestellt worden, in denen sich die Folgerungen aus der angeführten Theorie der Ketten gut bestätigt haben. Zu den endothermen umkehrbaren Ketten gehört nach den Untersuchungen von Jahn z. B. eine solche, welche aus einer Kupferelektrode in Kupferacetat- und einer Bleielektrode in Bleiacetatlösung besteht nach dem Schema:

\section{$\mathrm{Cu} \mid \mathrm{Cu}$ Ac. $\leftarrow$ Pb Ac. $\mid \mathrm{Pb}$.}

Der Pfeil gibt die Richtung des Stromes vom Blei durch die Flüssigkeiten zum Kupfer an; $\mathrm{Pb}$ löst sich auf und $\mathrm{Cu}$ wird ausgeschieden, wenn die Kette Strom liefert. Die chemische Energie der Kette reicht aber nicht aus, um alle erzeugte elektrische Energie zu decken. Die Kiette kühlt sich also bei Stromschluß ab, oder wenn sie isotherm arbeiten soll, so muß man ihr Wärme zufübren. In solchen Ketten wird daher Wärmevorrat der Kette selbst oder von auben aufgenommene Wärmeenergie zugleich mit der Umsetzung der chemischen Energie derselben in elektrische Energie umgewandelt. Die Messungen ergaben in dem angeführten Beispiel folgendes: Die chemische Energie der Kette ist gleich der Differenz der Verbindungswärmen beider Metalle mit der Essigsäure, für $1 \mathrm{~g}$-Mol. derselben gleich 16,523 g-cal. Die Kraft dieses Elementes wurde bei $20^{\circ}$ zu 0,47643 Volt gemessen und muß für den Strom 1 mit 46,12 zur Umrechnung in Wärmeenergie multipliziert werden. Man erhält für die elektrische Energie des Elementes demnach den Wert $46,12 \times 0,47643=21,96 \mathrm{~g}$-cal. Es müssen also $21,96-16,523 \mathrm{~g}$-cal aus dem Wärmevorrat desselben genommen bzw. bei isothermem Verlauf des Prozesses von außen zugeführt werden. Die Gleichung (2) ergibt für $20^{\circ}$ :

$$
E=\frac{16,523}{46,12}+(273+20) \frac{d E}{d T}=0,47643,
$$

woraus $\operatorname{sich} \frac{d E}{d T}=4,32 \cdot 10^{-4}$ berechnet. Im Versuch wurde 
für den Temperaturkoeffizienten der Wert $3,85 \cdot 10^{-4}$ gefunden. Noch besser stimmt die Rechnung mit der Beobachtung bei der Kette :

$$
\mathrm{Pb}\left|\mathrm{PbSO}_{4}\right| \mathrm{CuSO} \mathrm{O}_{4} \mid \mathrm{Cu} .
$$

l)er Strom geht in derselben rom $\mathrm{Pb}$ zum Cu. Die Verbindungswärme $\mathrm{Pb}, \mathrm{SO}_{4}$ ist 73,80 , diejenige von $\mathrm{Cu}, \mathrm{SO}_{4}$ ist 55,96 , die Differenz also 17,84. Die Kette besitzt bei $20^{\circ}$ eine $\mathrm{Kraft}$ von 0,61 Volt, ibr Temperaturkoeffizient ist gleich $7,7 \cdot 10^{-4}$. Die Gleichung (2) ergibt also für diesen Fall:

$$
E=\frac{17,84}{46,12}+293 \cdot 7,7 \cdot 10^{-4}=0,6115 \text { Volt } .
$$

Die der elektrischen Energie äquivalente Wärmemenge $0,61 \times 46,12=28,3$ ist also viel größer als die chemische, 17,84 , und die Differenz gleich 10,29 muß demnach aus einem Wärmevorrat entnommen werden.

In diesen Beispielen ist der chemische Prozeß ein exothermer, aber er reicht nicht aus, die elektrische Energie zu liefern. Die Kette arbeitet im ganzen daher endotherm.

Schon vor diesen Untersuchungen Jahns war von F. Bra un ${ }^{1}$ ) eine Kette konstruiert worden, in welcher der chemische Prozeß im ganzen ein endothermer ist. Sie besteht aus Cadmium und Eisen in ihren Sulfaten nach dem Schema:

$$
\mathrm{Cd}\left|\mathrm{Cd} \mathrm{SO} \mathrm{O}_{4}\right| \mathrm{FeSO}_{4} \mid \mathrm{Fe} \text {. }
$$

Der Strom fließt in dieser Kette rom Cadmium zum Eisen, Cadmium geht in Lösung und Eisen wird abgeschieden. Die Lösungswärme des Cadmiums ist 179,6 und die von Eisen 186.4. In diesem Falle absorbiert der chemische Prozeß 7,4 Wärmeeinlieiten, und trotzdem wird elektrische Energie erzeugt, die ausschließlich aus dem Wärmevorrat des Systems und der Umgebung genommen wird.

Es existiert also, wie aus diesen Beispielen folgt, in der Voltaschen Kette ein sehr wechselndes Verbältuis zwischen chemischer und elektrischer Energie, das aber durch die Formel (2) voll-

1) Wied. Ann. 5, 16 u. 17. 
ständig dargestellt werden kann. In dem letzteren Falle der Cd Fe-Kette ist $U$ negativ, $\frac{d E}{d T}$ positiv, und da die Kette bei $20^{\circ}$ etwa 0,1 Volt Kraft besaß, so würde die Gleichung (2) lauten:

woraus

$$
0,1=-\frac{7,4}{46,12}+293 \frac{d E}{d T},
$$

$$
\frac{d E}{d T}=+8,889 \cdot 10^{-4}
$$

folgen wïrde.

Es werden in diesem Falle 0,1.46,12 = 12,012 Wärmemengen aus dem Vorrat des Systems entnommen, um den chemischen und elektrischen Prozeß zu unterhalten.

In den angeführten Versuchen voll Jahn wurde die Kette in ein Bunsensches Eiskalorimeter gesetzt und die abgegebene oder anfgenommene Wärme während der Stromerzeugung gemessen. Führt man die elektrische Energie durch eine Leitung nach anßen, was durch große Widerstände im Verhältnis zum Widerstand der Kette fast vollständig geschehen kann, so kann man sie nach bekannten Methoden messen. In diesem Falle ist dio positive oder negative chemische Wärme der Kette $U$ gleich der äußeren Stromwärme $W$ plus der an das Kalorimeter abgegebenen Wärme $C$, welche positiv oder negativ sein kann. Also:

$$
+U=\mathrm{U}+\mathrm{C} \text {. }
$$

Sind $U$ und $($ ' positiv, also $V=W+C$, so ist die Kette eine exotherme, die sich erwärnt; ist $U$ positiv and $C$ gleich Null, also $I=C$ wie beim Daniell, so ist die Kette eine exotherme mit konstanter Temperatur. Ist $U$ positiv und $C$ negativ und ist $W>C$, so ist die Kette eine endotherme mit exothermem, chemischem Prozeßs. Ist $U$ negativ und $C$ negativ, so muß $C>W$ sein, wenn die Kette Strom liefern soll, da $-U=W-C$ wird. Die Kette ist eine endotherme mit endothermem, chemischem Prozeß.

Eine befriedigende anschauliche theoretische Vorstellung von dem inneren Zusammenhang zwischen chemischer Affinität und elektrischer Kraft der Atome, die in Aktion treten, ist bisher für den hier stattfindenden energetischen Prozel noch nicht gegebell worden. Doch ist es Nernst') gelungen, von den gleich

1) Siehe Nernst, Theoretische Chemie, 1900. 
zu behandelnden lionzentrationsketten ausgehend, eine umfassende Theorie aller elektrischen líetten aufzustellen, die auf den Gesetzen der Osmose und der Ionenlehre berulit.

Wir gelangen nun zu denjenigen Ketteı, in welchen im ganzen gar keine chemische Änderung stattfindet, in denen also $U=0$ ist. Das sind die von Helmholtz zuerst erfundenen Konzentrationsketten. Die einfachste Form derselben besteht aus zwei gleichen Metallelektroden, welche in einer konzentrierten und verdünnten Lösung dieses Metalles stehen, die durch einen mit dieser Lösung gefüllten Heber verbunden sind; z. B. Zinkelektroden in Zinksulfat nach dem Schema:

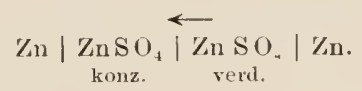

Der Strom fließt bei Verbindung der Zinkelektroden in der Richtung des Pfeiles von der verdünnten zur konzentrierten Lösung. An der Elektrode der verdünnten Lösung löst sich Zink auf und an der Elektrode der konzentrierten Lösung scheidet sich ein gleiches Äquivalent Zink ab, so daß die chemische Energie der Kette gleich Null ist. Es gilt also für eine solche Kette die Gleichung:

$$
E=T \cdot \frac{d E}{d T}
$$

Es ist klar, dab der Temperaturkoeffizient einer Konzentrationskette ein positiver sein muß und daß die elektromotorische Kraft derselben proportional der absoluten Temperatur steigt.

In der Konzentrationskette der angegebenen Art kommen drei Kontaktpotentiale zur Wirkung: erstens die der Elektroden gegen die Lösungen und zweitens das der Lösungen gegeneinander. Das letztere ist das Wesentliche in einer solchen Kette. Man kann aher auch Konzentrationsketten herstellen, in welchen nur die Kontaktpotentiale der Lösungen in Betracht kommen, während sich die Elektrodenpotentiale als gleich und entgegengesetzt aufheben. Solche Ketten sind von Nernst konstruiert und untersucht worden.

Die Potentialdifferenz zwischen zwei Lösungen eines Elektrolyten verschiedener Konzentration läßt sich auf verschiedene 
Weise theoretisch ableiten. $\quad$. Helmholtz hat für die erwähnte Kette mit Zink in Zinksulfat auf thermodynamischem Wege die Kraft berechnet, indem er den Lampfdruck der verdünnten und konzentrierten Lösung hierzu velwendete, und hieraus die Wärmemenge erhielt, welche nötig ist, um das auf 1 Mol. Salz der verdünnteren Lösung kommende Wasser in die konzentriertere Lösung überzuführen. Hierbei denkt man sich so große Mengen der Lösungen, daß sich die Konzentrationen dabei nicht merklich ändern. Der Dampfdruck einer Lösung nimmt bekanntlich bei zunehmender Konzentration ab. Es ist also Arbeit erforderlich, um ibn zu vermindern. Diese aus der Verdampfungswärme zu berechnende Arbeit ist gleich der elektrischen Energie, welche denselben Ausgleich der Konzentrationen zwischen den beiden Lösungen hervorbringen würde, und dividiert man diese durch die dem Faradayschen Gesetz entsprechende Elektrizitätsmenge, welche zur Elektrolyse von 1 Mol. des Elektrolyten erforderlich ist, so erhält man die elektromotorische Kraft der Kette. Anschaulicher ist die Theorie von Nernst ${ }^{1}$ ), welche von dem osmotischen Druck der Lösungen und der Beweglichkeit der Ionen der Elektrolyte ausgeht. Man denke sich einen Diffusionszylinder der Lösung eines Elektrolyten, an dessen einem Ende die lionzentration eine höhere ist als am anderen, so werden infolge der Diffusion die Moleküle des Elektrolyten sich vom Orte höherer nach denen niederer Konzentration bewegen. Nehmen wir nun den einfacheren Fall an, daß es sich nur um solche Konzentrationen handelt, bei denen alle Moleküle in ihre Ionen dissoziiert sind, so wissen wir aus dem Verhalten der lonen bei der Elektrolyse, daß sie eine verschiedene Beweglichkeit besitzen. Infolgedessen wird entweder das positive oder das negative Ion eines Moleküls das Bestreben haben, dem anderen bei der Diffusion vorauszueilen. Da aber die Ionen sich innerhalb der Lösung auch in dem dissoziierten Molekül nicht voneinander trennen können, so wird bei dieser Bewegung die Geschwindigkeit des einen Ions verzögert, die des anderen beschleunigt and ebenso die Geschwindigkeiten, mit denen sich die elektrischen Ladungen der Ionen bewegen. Es muß daher eine elektrische Potentialdifferenz entstehen, welche aus

1) Elektromotorische Wirksamkeit der Ionen. Zeitschr. f. physik. Chem. 4, 129 (1889). 
der Beschleunigung der Bewegung des einen und der Verzögerung der Bewegung des anderen Ions erfolgt. Ist die Beweglichkeit des positiven Ions, des Kations, eine größere als die des negativen, des Anions, so nimmt das verdünnte Ende des Diffusionszylinders positive, das konzentriertere Ende desselben negative Spannung an. Ist dagegen die Beweglichkeit des negativen Ions, des Anions, eine größere als die des positiven, des Kations, so ist es umgekehrt.

In Fig. 9 ist die Wanderung der Ionen in einem elektrischen Strom nach Ostwald dargestellt.

Fig. 9.

a.
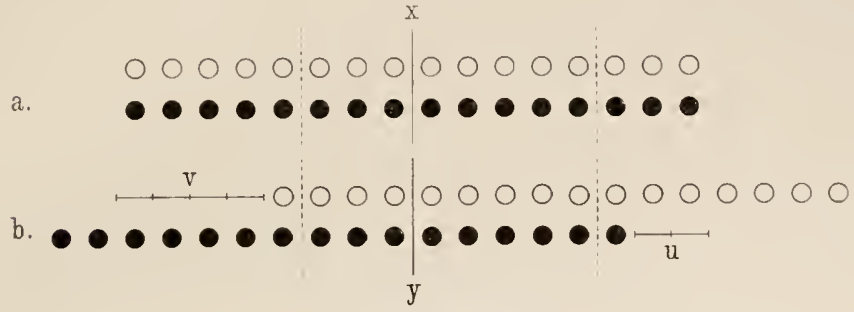

Wanderung der Ionen.

In der Fig. 9 stellen die schwarzen und weißen Kügelchen die beiden Ionen eines Moleküls (oder besser Äquivalents, das hier dem Molekül gleichgesetzt sein möge) dar, in a vor der Durchleitung des Stromes, in $b$ während der Durchleitung bei der Elektrolyse. In a sind die beiden Ionen, welche übereinander stehen, zu einem Molekül verbunden, aber nach Clausius und Arrhenius in bereits dissoziiertem Zustande. Die $x y$-Ebene teilt die Lösung in zwei gleiche, gleichkonzentrierte Hälften; die punktierten Striche stellen die Elektroden des Stromes dar. In $b$ sieht man die Wanderungen der beiden Ionen nach den Elektroden. Ihre Geschwindigkeiten sind nicht gleich, sondern meist recht verschieden, was zuerst von Hittorf (1853) nachgewiesen worden ist. In dem gedachten Bilde wandern die weißen Kügelchen, welche die Anionen darstellen mögen, schneller als die schwarzen, welche Kationen bedeuten, denn während bis zu dem gedachten Zeitpunkt vom Anfang der Elektrolyse vier weiße die Mittellinie $x y$ durchwandert haben, haben sich nach der anderen Richtung nur zwei schwarze hindurchbewegt. $v$ ist daher die Verschiebung der Anionen, $u$ diejenige der Kationen. Man erkennt 
aber, daß an den beiden Elektroden gleichviel lonen, nämlich $4+2=6$ freigeworden sind, wie es das Faradaysche Gesetz verlangt. Dagegen muß infolge der verschieden schnellen Ionenbewegung die Konzentration der beiden Lösungshälften verschieden geworden sein. Von dem Salz sind rechts zwei, links vier Äquivalente durch Elektrolyse verschwunden. Diese Verluste verhalten sich also wie die Wanderungsgeschwindigkeiten (Beweglichkeiten) der beiden Ionen.

Nennt man die Beweglichkeiten des Kations $u$ und die des Anions $v$, so erbält man durch die oben angegebenen elektrolytischen Versuche das Verhältnis von $u: v$, also auch die Werte $\frac{u}{u+v}$ und $\frac{v}{u+v}$, welche man die Hittorfschen Wanderungsoder Überführungszahlen der Ionen genannt hat. Stellt man sich eine Lösung eines Elektrolyten von $1 \mathrm{~cm}$ Länge und $1 \mathrm{~cm}^{2}$ Qnerschnitt vor und leitet durch diesen einen Strom von 1 Volt, so kann man aus der Leitfähigkeit der Lösung die Stromstärke in Ampere berechnen, und findet daraus, wieviel Grammäquivalente sich in einer Sekunde an clen Endflächen des Kubikzentimeters abscheiden müßten. Man weiß nun, daß die Leitung der Elektrizität in der Lösung eines Elektrolyten nur durch die Bewegung der Ionen geschieht. Man kamı daher die Leitfähigkeit einer Lösung $\lambda=u+v$ setzen, wenn alle Moleküle in ihre Ionen dissoziiert sind, was bei einer sehr verdünnten Lösung der Fall ist. Drückt man $\lambda$ in bekannten Einheiten aus, wenn $1 \mathrm{~g}$ Äquivalent in 1 Liter Flüssigkeit gelöst ist, so erhält man auch die entsprechenden Werte für $u$ und $v$ Es sind nach Kohlrausch die Werte für $u$ und v bei einer Zahl von Ionen folgende:

\begin{tabular}{|c|c|c|c|c|c|c|c|c|c|c|c|c|c|c|c|}
\hline \multicolumn{8}{|c|}{ น. $10^{7}$ (Kationen) } & \multicolumn{8}{|c|}{ v. $10^{7}$ (Anionen) } \\
\hline $\mathrm{H}$ & . . & . & . & . & & & - 300 & $\mathrm{OH}$ & . . & . & . & . & & & 165 \\
\hline $\mathrm{K}$ & . . & . & . & . & . & & . 60 & $\mathrm{Cl}$. & . . & . & . & . & 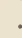 & . & 63 \\
\hline $\mathrm{Na}$ & . . & . & . & . & . & • & 41 & $\mathrm{Br}$ & . . & . & . & . & . & & 62 \\
\hline Li . . & . . & . & . & . & . & • & 33 & J . & . . & . & . & . & & & 62 \\
\hline $\mathrm{NH}_{4}$. & . . & . & 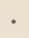 & . & & & 60 & $\mathrm{NO}_{3}$ & . . & . & . & & & & 58 \\
\hline $\mathrm{Ag}$. & . . & . & . & . & & & · $\quad 52$ & $\mathrm{ClO}_{3}$ & . & . & . & & & & 52 \\
\hline $1 / 2 \mathrm{Ca}$ & . & . & . & . & & & - $(46)$ & $\mathrm{CHO}$ & $f_{2}$. & . & . & & & & 44 \\
\hline $1 / 2 \mathrm{Mg}$ & . & . & . & - & & & - (46) & $\mathrm{C}_{2} \mathrm{H}_{3}$ & ${ }_{3} \mathrm{O}_{2}$ & . & . & & & & 33 \\
\hline $1 / 2 \mathrm{Zn}$ &. & 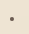 & & & & & $(46)$ & $1 / 2 \mathrm{~S} \mathrm{C}$ & $\mathrm{O}_{3}$. & & & & & & $(66)$ \\
\hline $1 / 2 \mathrm{Cu}$ & . & ${ }^{\circ}$ & & & & & $(48)$ & & & & & & & & \\
\hline
\end{tabular}


Mau ersieht hieraus, daß unter den Kationen das H-Ion $\left(\mathrm{H}^{+}\right)$ die größte Beweglichkeit besitzt, unter den Anionen das Hydroxylion $\left(\mathrm{OH}^{-}\right)$. Kalium - und Chlorionen besitzen ungefähr die gleiche Beweglichkeit. Eine gesetzliche Beziehuug der Ionenbeweglichkeiten zu auderen Konstanten der Atome und Moleküle del Elektrolyte ist nicht ermittelt, nur deutet die große Beweglichkeit des H-Ions darauf hin, daß die Geschwindigkeit der Moleküle im Gaszustand und die Reibung in dem Lösungsmittel zu den Variablen der gesuchten Funktion gehören werden.

Kehren wir nun zu der Betrachtung eines Diffusionszylinders wieder zurück, wie er durch Fig. 10 dargestellt ist, dessen Ende $C$ die stärkere, $c$ die schwächere Konzentration eines Elektrolyten enthalten möge, z. B. Salzsäure, ClH. Stellen wir uns vor, daß die dissoziierten Moleküle $\mathrm{H}^{+} \mathrm{Cl}-$ in der Richtung des Pfeiles sich bewegen, so hat das positive Ion $\mathrm{H}^{+}$infolge seiner größeren Beweglichkeit das Bestreben, dem negativen Ion $\mathrm{Cl}^{-}$bei der Diffusion voranzueilen.

Fig. 10 .

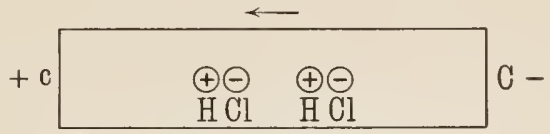

Diffusionszylinder und Diffusionspotential.

Da aber die beiden Ionen durch die chemische Affinität der A tome Cl und $\mathrm{H}$ miteinander verbunden sind, so können sie sich nicht trennen, und das schnellere $\mathrm{H}^{+}$-Ion schleppt das langsamere $\mathrm{Cl}-$-Ion hinter sich ber, wie es in der Fig. 10 bildlich wiedergegeben ist. Diese Vorstellung, die nur als Anschauungsbild gelten soll, versinnlicht die Entstehung einer elektrischen Spannung an den Enden des Diffusionszylinders, in dem die positiven Ionen sich dem verdünnten, die negativen dem konzentrierten Ende desselben zuwenden. Es tritt ein Diffusionspotential auf. Bei nicht dissoziierten Molekülen eines Elektrolyten oder bei Molekülen eines Nichtelektrolyten würde eine derartige Ordnung der Lage der'selben in einem Diffusionszylinder nicht eintreten. Es wird also gewissermaßen bei der Diffusion und Osmose eines dissoziierten elektrolytischen Moleküls eine ungeordnete Bewegung in eine geordnete umgesetzt. Rein energetisch betrachtet ist es Wärmeenergie, welche sich bei diesem Vorgange in elektrische verwaudelt; denn bei jeder Diffusion oder Osmose wird Wärme absorbiert, und in vorliegendem Falle erscheint ein Teil dieser Wärme als elektrische Energie. Ungeordnete Wärmebewegung wird in eine geordnete 
Bewegung umgesetzt. Die Potentialspannung an den Enden des Diffusionszylinders kann aber nicht ohne weiteres allein zur Stromerzeugung verwendet werden. Schließt man den Diffusionszylinder selbst zum Kreise, so hat man gleiche und entgegengesetzte Spannungen, die sich aufheben. Oder taucht man in die Enden desselben Metallelektroden, so gesellen sich zum Potential des Zylinder's die Elektrodenpotentiale hinzu, so daß man das erstere nicht an sich feststellen kann. Es bedarf daher eines Kunstgriffes, um eine reine Konzentrationskette zusammenzustellen, deren Kraft nur von dem Potential des Diffusionszylinders helrührt, also eine rein osmotische Flüssigkeitskette. Eine solche hat Nernst z. B. in folgender Weise zusammengesetzt, nach dem Schema:

$$
0,1 \mathrm{KCl}|0,01 \mathrm{KCl}| \stackrel{\leftarrow}{0}, 01 \mathrm{HCl}|0,1 \mathrm{HCl}| 0,1 \mathrm{~K} \mathrm{Cl} \text {. }
$$

In dieser Kombination herrscht die Potentialdifferenz $0,01 \mathrm{HCl}$ $\mid 0,1 \mathrm{HCl}$ vor, denn die Differenz $0,01 \mathrm{KCl} \mid 0,01 \mathrm{H} \mathrm{Cl}$ ist gleich und entgegengesetzt der von $0,1 \mathrm{HCl} \mid 0,1 \mathrm{KCl}$, da es nicht auf die absolute Konzentrationsdifferenz, sondern auf das Verbältnis der Konzentrationen zueinander ankommt, und die Differenz $0,1 \mathrm{KCl} \mid 0,01 \mathrm{KCl}$ kann man nahezu als Null annehmen, weil die Ioneubeweglichkeiten von $\mathrm{K}^{+}$und $\mathrm{Cl}^{-}$(siehe Tabelle) nahezu dieselben sind. Die Konzentrationen, welche angegeben sind, sollen molekulare bedeuten. Um einen Strom von dieser Kette abzuleiten, könnte man zwei unveränderliche Metallelektroden, z. B. aus Platin in die Endglieder eintauchen, deren Potentiale sich aufheben würden. Da solche aber polarisierbar sind, so ist es zweckmäßiger, sich hierzu unpolarisierbarer Elektroden zu bedienen, am besten in diesem Falle der Quecksilber-Kialomelelektroden, welche mit $0,1 \mathrm{~K} \mathrm{Cl}$ gefüllt werden (siehe oben S. 4).

Die Rechnung, welche Nernst auf Grund seiner os motischen Theorie der Konzentrationsketten ausgeführt hat, hat für die Potentialdifferenz zwischen zwei Lösungen desselben Elektrolyten von verschiedener Konzentration folgendes Resultat ergeben. Beschränkt man die Betrachtung auf verdünnte Lösungen, in denen alle Moleküle als dissoziiert angenommen werden können, setzt man statt der molekularen Konzentrationen der Lösungen die osmotischen Drucke derselben $p_{1}$ und $p_{2}$ (bekanntlich sind die 
osmotischen Drucke den molekularen Konzentrationen der Lösungen proportional), ist die absolute Temperatur der Lösungen $T$ und $K$ eine Konstante, so ist die Potentialdifferenz zwischen den beiden Lösungen:

$$
\pi=K \cdot T \cdot \frac{u-r}{u+v} \log n a t \frac{p_{1}}{p_{2}} .
$$

Hieriu bezeichnen $u$ und $v$ die Beweglichkeiten des Kations und des Anions und die Konstante $K$ ist bei der Berechnung der Potentiale in Volts gleich $8,60 \cdot 10^{-5} \mathrm{zu}$ setzen. Bedient man sich ferner des gewöhnlichen Logarithmus statt des natürlichen, so hat man den Wert durch 0,4343 zu dividieren, und man erhält hiernach:

$$
\pi=0,000198 \cdot T \cdot \frac{u-v}{u+v} \log \frac{p_{1}}{p_{2}} \text { Volt }
$$

Setzen wir $p_{1}>p_{2}$ und ist $u>v$, so ist $\pi$ positiv und der Strom geht von der konzentrierten Lösung 1 zur verdünnteren 2. Ist dagegen $v>u$, so hat der Strom die umgekehrte Richtung. Die Valenz der Ionen sei gleich. Zieht man in der angegebenen Konzentrationskette (S.34) von Nernst auch die Potentialdifferenz zwischen $0,1 \mathrm{KCl} \mid 0,01 \mathrm{KCl}$ in Betracht, und nennen wir die Beweglichkeiten in dem einen Elektrolyten $(\mathrm{ClH}) u_{1}$ und $v_{1}$ und in dem anderen $(\mathrm{KCl}) u_{2}$ und $v_{2}$, so erhalten wir bei einer so zusammengesetzten Kette für die elektromotorische Kraft:

$$
E=K \cdot T \cdot\left(\frac{u_{1}-v_{1}}{u_{1}+v_{1}}-\frac{u_{2}-v_{2}}{u_{2}+v_{2}}\right) \log n a t \frac{p_{1}}{p_{2}} .
$$

da in beiden Elektrolyten $p_{1}$ und $p_{2}$ denselben Wert haben. Bei den oben erwähnten Konzentrationsketten, in welchen die metallischen Elektroden in die beiden ungleich konzentrierten Lösungen

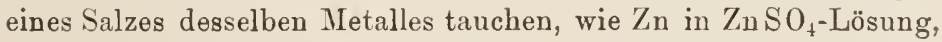
kommen noch die Kontaktpotentiale der Elektroden in Betracht, und man erhält nach Nernst die Gleichung:

$$
E_{k}=K \cdot T \cdot \frac{2 v}{u+v} \log n a t \frac{p_{1}}{p_{2}} .
$$

In diesem Falle bestehen die Elektroden aus dem Kation des Elektrolyten und sind in bezug auf das Kation umkehrbar. Man erhält in bezug auf das Anion des Elektrolyten umkehrbare Elek- 
troden, wenn das Anion sich in einer festen scliwer löslichen Verbindung auf der Oberfläche einer Metallelektrode befindet, z. B. bei den oben beschriebenen Quecksilber-Kalomelelektroden gegen verschieden konzentrierte Lösungen eines Chlorides. Die Kraft eiuer solchen Kette ist:

$$
E_{a}=K \cdot T \cdot \frac{2 u}{u+v} \log n a t \frac{p_{1}}{p_{2}} .
$$

Alle Konzentrationsketten sind, wie man leicht einsieht, umkehrbare, d. h. durch den entgegengesetzten Strom von derselben Intensität könneu sie wieder in den anfänglichen Znstand zurückgebracht werden. Man erkennt auch aus den Gleichungen (4) bis (8), daß die Kraft dieser Ketten der absoluten Temperatur proportional sein mußs, wenn man die übrigen Gröben außer $T$ konstant setzt. Indessen sind die Beweglichkeiten der lonen eines Elektrolyten in einer Lösung keineswegs absolut konstant bei wechselnder Temperatur, doch ist ihr Temperaturkoeffizient nur ein verhältnismälig geringer, und für bisher untersuchte Jonen ein nicht sehr verschiedener. Wäre er für alle Ionen ein gleicher, so würden die Werte der Formeln (4) bis ( 8 ) dieselben bleiben, da in ihnen nur die Verhältnisse von $u: v$ vorkommen. Nach Versuchen von Fr. Kohlrausch ${ }^{1}$ ) sind die Temperaturkoeffizienten für Beweglichkeiten $\mathrm{Na}^{+}, \mathrm{Cl}^{-}$und $\mathrm{H}^{+}$folgende: Beweglichkeiten bei $18^{\circ} \mathrm{C}: u_{\mathrm{Na}}=43,55, \quad{ }^{\mathrm{Cl}}=65,44, \quad u_{\mathrm{H}}=318$, Temperaturkoeffizienten: ${ }_{\mathrm{Na}}=0,0244,{ }{ }_{\mathrm{Cl}}=0,0210,{ }{ }_{\mathrm{H}}=0,0153$.

Man sieht, daß diese Koeffizienten für die genannten Ioneu annähernd gleich 0,02 sind. Bei genaueren Berechnungen kann man sie allerdings nicht veruachlässigen. Es ist daher von Interesse $\mathrm{zu}$ berechnen, wie weit die Kraft solcher Ketten von der Proportionalität mit der absoluten Temperatur abweicht, da wir auch im folgenden davon Gebrauch machen werden. Eine von Nernst (l. c.) untersuchte und berechnete Kette aus $\mathrm{NaCl}$, von den Konzentrationen 0,125 und $0,0125 \mathrm{~g} \ddot{\mathrm{Aq}}$. pro Liter mit Hg-Kalomelelektrode abgeleitet, ergibt, nach der Formel:

$$
E=0,860 T \frac{2 u}{u+v} \log n a t \frac{p_{\mathrm{I}}}{p_{2}} \cdot 10^{-4} \text { Volt }
$$

$\left.{ }^{1}\right)$ Über die T'emperaturkoeffizienten der Ionen im Wasser usw. Sitzungsber. d. Berl. Akad. 1902, S. 572, 29. Mai. 
berechnet, für $18^{\circ} \mathrm{C}$ den Wert 0,046 Volt, und für $32^{0} \mathrm{C}$ den Wert 0,0491 Volt, während nach Annahme der Proportionalität mit $T$ sich 0,0484 ergeben würde. Die Abweichung beträgt in diesem Falle etwa + 1,43 Proz.

Für eine Kette von $\mathrm{ClH}$, aus 0,1 und $0,01 \mathrm{~g}$ Äq. zusammengesetzt, erhielt man bei $18^{\circ} \mathrm{C}$ den Wert $E_{18}=0,0956$ und bei $32^{\circ} \mathrm{C} E_{32}=0,09895$. In Falle der Proportionalität erhielt man $E_{32}=0,1002$, also ist die Abweichung etwa $-1,23$ Proz.

Man sieht, daß diese Abweichungen verhältnismäßig gering sind. Von Interesse ist nun auch der Fall, daß zwei Lösungen verschiedener Elektrolyte miteinander in Berührung stehen, der von Planck theoretisch behandelt ist. Nimmt man der Einfachheit halber zwei Lösungen von gleicher molekularer Konzentration an, nenut die Beweglichkeiten der Ionen des einen Elektrolyten $u_{1}$ und $v_{1}$, die des anderen $u_{2}$ und $v_{2}$, so ist die Potentialdifferenz zwischen beiden Lösungen:

$$
\left.\pi=0,000198 . T \cdot \log \frac{u_{1}+v_{2}}{u_{2}+v_{1}} \text { Volt }^{1}\right),
$$

oder für $18^{\circ} \mathrm{C}(T=273+18=291)$ :

$$
\pi=0,0577 \log \frac{u_{1}+v_{2}}{u_{2}+v_{1}} \text { Volt. }
$$

Aúch diese Potentiale steigen daher proportional mit der absoluten Temperatur (abgesehen von der Änderung der Ionenbeweglichkeiten). Ferner sind von Nernst und Riesenfeld2) Potentialdifferenzen an der Grenzfläche zweier Lösungsmittel nachgewiesen worden, in denen ein Elektrolyt gelöst ist. Wenn man z. B. in ein U-Rohr unten Phenol und in beide Schenkel oben Wasser einfüllt, in welchem ein Elektrolyt gelöst ist, so verteilt sich derselbe in bestimntem Verhältnis auf die beiden Lösungsmittel. Bei Durchleitung eines Stromes treten an den beiden Grenzflächen Elektrolysen auf, die man z. B. bei Lösung

$\left.{ }^{1}\right)$ Bei vollkommener Dissoziation der Moleküle und gleicher Valenz ihrer Ionen.

$\left.{ }^{2}\right)$ Nernst und Riesenfeld, Über elektrolytische Erscheinungen an der Grenzfläche zweier Lösungsmittel, Ann. d. Physik (4) \&, 600; E. H. Riesenfeld, Konzentrationsketten mit nicht mischbaren Flüssigkeiten, daselbst, S. $616,1902$. 
von $K^{\prime} J_{3}$ an den Grenzflächen durch Farbenänderungen erkennen kann. Es geht daraus hervor, dab an den Trenuungsflächen der' Lösungsmittel ein Potentialsprung existiert. Der Elektrolyt velteilt sich auf die beiden Lösungsmittel in einem Verhältnis, das durch den Teilungskoeffizienten ausgedrückt wird. Nernst nimmt an, daß nicht bloß die Moleküle des Elektrolyten, sondern auch die Ionen desselben einen besonderen voneinander verschiedenen Teilungskoeffizienten besitzen. In dem einfachsten Falle eines binären Elektrolyten, der sich auf zwei Lösungsmittel $L_{1}$ und $L_{2}$ verteilt, sind daher drei Teilungskoeffizienten zu unter-

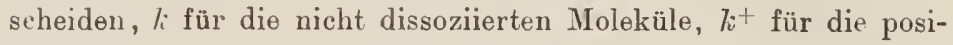
tiven und $k^{-}$für die negativen Ionen. Sind $k^{+}$und $k^{-}$verschieden groß, so ist die Zahl der positiven und negativen Ionen in jedem der beiden Lösungsmittel eine verschiedene, und da im Inneren jedes Lösungsmittels keine freie Elektrizität sein kann, so entsteht an der Trennungsfäche derselben eine Doppelschicht, d. h. eine Potentialdifferenz zwischenden beiden Lösungsmitteln (Phasen).

Wenn $c^{+}$und $c^{-}$die Konzentrationen der Kat- und Anionen in $L_{1}$ und $\gamma^{+}, \gamma^{-}$die in $I_{2}$ sind, $m_{1}$ und $m_{2}$ die Wertigkeiten derselben, und $R$ die Gaskonstante bedeutet, so ist nach Nernst diese Potentialdifferenz:

$$
\pi=\frac{R T}{m_{1}} \log n a t \frac{k^{+} \cdot \gamma^{+}}{c^{+}}=-\frac{R T}{m_{2}} \log n a t \frac{k^{-} \cdot \gamma^{-}}{c^{-}} .
$$

Riesenfeld hat Konzentrationsketten mit nicht mischbaren Flüssigkeiten, z. B. Phenol und Wasser, in denen Halogensalze gelöst waren, zusammengesetzt, deren Strom sich aus den angegebenen Grenzkräften erklärt. I)a man zwei nicht (oder nur wenig) mischbare Flüssigkeiten als zwei verschiedene Phasen ansehen kann, so hat man diese elektrischen Potentiale auch Phasengrenzkräfte genannt. 


\section{Drittes Kapitel.}

\section{Elektrische Vorgänge in Nerven und Muskeln in ihrer Beziehung zur Erregung, Reizleitung und Kontralition.}

Die in dem ersten Kapitel berichteten Tatsachen geben, abgesehen von einigen noch später zu behandelnden Erscheinungen ${ }^{1}$ ), im großen und ganzen den Stand der Elektrophysiologie in dem Zeitpunkte an, als ich im Jahre $1866 \mathrm{mir}$ die Frage vorlegte, mit welcher Geschwindigkeit der durch die negative Schwankung wahrnehmbare Prozeß der Zustandsänderung im Nerven sich fortpflanze ${ }^{2}$ ). Wenn dieser Vorgang in seiner Ursacbe gleichbedeutend ist mit dem im Nerven bei Reizung oder natürlicher Tätigkeit fortgeleiteten Impulse, welcher im Nuskel dio Kontraktion, in den Zentren die Empfindung auslöst, so mub diese Geschwindigkeit mit derjenigen übereinstimmen, welche Helmboltz, wie oben angeführt, für die Nervenerregung gefunden hatte. Ein solches Resultat mubte nicht nur ein Kriterium für die Bedeutung der elektrischen Prozesse in den Organen ergeben, sondern mußte auch Aufschluß gewähren über die Form, in welcher die Erregungsimpulse in den Nerven und Muskeln ablaufen, worüber wir aus der bloßen Beobachtung der Muskelzuckungen oder der subjektiven Empfindungen bei Nervenreizung nichts Bestimmtes erfahren können. Die Erwartungen sind nicht

1) Insbesondere die Polarisationen in Nerven und Muskeln, der sogenanite Elektrotonus.

$\left.{ }^{2}\right)$ Erste vor]äufige Mitteilung: „Die Fortpflanzungsgeschwindigkeit der negativen Schwankung im Nerven, Zentralblatt für die medizinischen Wissenschaften 1866 , S.597. - Über den zeitlichen Verlanf der negativen Schwankung des Nervenstromes, Monatsber. d. Berl. Akad. 1867 , S. 72 . - Über den zeitlichen Verlauf der negativen Schwankung des Muskelstromes, daselbst, S. 440. - Über den zeitlichen Verlauf der negativen Schwankung, Pflüg. Arch. 1, 179-207 (1868). — Über den Erregungsvorgang im Nerven - und IIuskelsysteme. Heidelberg 1871. $240 \mathrm{~S}$. 
getäuscht worden. Es wurde in diesen Untersuchungen gefunden, erstens, daß die elektrische Zustandsänderung in den Nerven sich mit derselben Geschwindigkeit fortpflanzt wie der Erregungsprozeß, zweitens, daß jeder einzelne Impuls, an der elektrischen Veränderung gemessen, einen wellenförmigen Verlauf von gewisser Dauer besitzt. Diesen durch einen momentanen Reiz hervorgerufenen, wellenartig sich fortpflanzenden Prozeß habe ich die "Reizwelle"1) genannt.

I)ie Methode der Untersuchung war im Prinzip folgende: Ein möglichst langer Nerv oder Muskel wird an einem Ende durch Induktionsströme gereizt, welche durch einen möglichst momeutanen Kontakt des primären Kreises eines Induktoriums entstehen. Am anderen Ende wird der Längsquerschnittstrom alugeleitet, um zum Galvanometer geführt zu werden. Dieser Kreis ist aber nicht dauernd geschlossen, sondern wird nur in bestimubaren Zeitintervallen nach der Reizung auf kurze Zeitdauer geschlossen. Der Längsquerschnittstrom in der Ruhe sei vollständig kompensiert. Schließung und Öffnung des Kreises wird also keine Ablenkung des Galvanometers verursachen. Findet aber eine momentane Reizung statt und bald darauf ein kurzer Schluß des Galvanometerkreises, so wird eine Ablenkung im Sinne der negativen Schwankung erscheinen, wenn sich in dem Zeitintervall zwischen Reizung und Nervenstromschluß die Reizwelle bis zur abgeleiteten Stelle fortgepflanzt hat. Vergrößert man dieses Intervall, so wird sie sich zuerst verstärken und dann bis zum Verschwinden wieder abnehmen, sobald die ganze Reizwelle die abgeleitete Stelle, und zwar, wie sich ergab, den abgeleiteten Längsschnittpunkt, überschritten hat. Versuche ganz derselben Art sind dann auch mit dem Muskel vorgenommen worden, wozu man einen möglichst langen, parallelfaserigen Muskel, z. B. den Musc. sartorius des Frosches, benutzen kann, nur muß man in diesem Falle die motorischen Nerven desselben durch vorherige

1) Diese Welle ist auch von einigen "Erregungswelle" genannt worden. Indessen ist meines Erachtens der Begriff der Erregung weiter zu fassen und auch noch auf andere Vorgänge als die elektrischen, z. B. auf die Kontraktion, auszudehnen. Andere haben diese Welle auch "Negativitätswelle" genannt (wegen तer Richtnng des Potentials), doch halte ich diesen Ausdruck nicht für exakt. 
liurare-Vergiftung ${ }^{1}$ ) des Tieres lähmen, damit sich die Erregung und Reizwelle nur durch die Muskelfasern fortpflanzt und nicht auf dem Wege der motorischen Nerven.

In Fig. 11 ist der benutzte Apparat, das Rheotom (oder Differentialrheotom), von oben gesehen, abgebildet und in Fig. 12 mit der Versuchsanordnung schematisch dargestellt. Da die nach einem einzelnen Reize erfulgenden Galvanometerausschläge sehr klein sind und daher eine genauere Beobachtung des zeitlichen Ablaufes, ins-

Fig. 11.

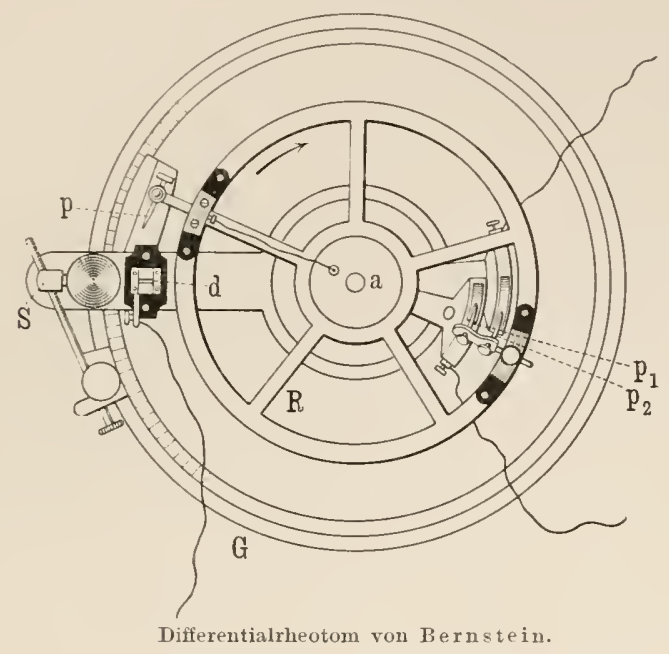

besondere die Bestimmung des Anfangspunktes nicht zulassen, so finden die Reizungen und Stromableitungen periodisch etwa fünf- bis zehnmal in der Sekunde statt. Das Rheotom besteht aus einem um die Achse $a$ rotierenden Rade, an dessen Peripherie etwa diametral gegenüber zwei isolierte Kontakte angebracht sind. Der eine derselben, der Reizkontakt $p$, ist eine harte Stahlspitze, welche unter etwa $45^{0}$ schräg nach unten gerichtet ist und über einen Metalldraht $d$ streift, um den primären Strom eines Induktoriums momentan zu schließen. Dieser Draht $d$ befindet sich anf einem

1) Das Kurare, amerikanisches Pfeilgift, lähmt die motorischen Nervenendigungen in den Muskeln, so daß Nervenreizung keine Kontraktion bewirkt, während direkte IInskelreizung normalen Erfolg hat. 
Schieber S, der sich durch einen Messingarm um die ganze Peripherie der Grundscheibe $G$ drehen läßt und durch eine senkrecht stehende Mikrometerschraube boch und niedrig gestellt werden kann, um den Momentankontakt bei der Rotation des Rades in der Richtung des Pfeiles einzustellen. Fine zweite horizontal liegende Nikrometerschraube erlaubt eine feinere Verschiebung des Kontaktes, den man auf einer Teilung der Grundscheibe ablesen kann. Dem Reizkontakt gegenüber stehen, isoliert voneinander, zwei besonderen drehbaren Messingarmen aufgesetzt, zwei längliche Quecksilbergefäße $Q Q$. In das Quecksilber tauchen möglichst oberflächlich zwei messerartig geschärfte, amalgamierte Kupferspitzen $p_{1}$ und $p_{2}$, welche vom Rade isoliert, aber miteinander leitend verbunden sind. Durch diese wird der Galvanometerkreis längere oder kürzere Zeit periodisch geschlossen, solange die Spitzen $p_{1} p_{2}$ über die Quecksilberoberfläche streifen. Diese Schließungszeit kann beliebig variiert werden, indem man die Quecksilbergefäße gegeneinander verschiebt. Sind die Kupferspitzen $p_{1} p_{2}$ amalgamiert und leicht die $\mathrm{Hg}$ - Oberfläche streifend eingestellt, so sind die Momente der Schließung und Öffnung in den $\mathrm{Hg}$-Gefäßen bei der Rotation sehr koustant, ohne daß das Quecksilber dabei in merkliche Bewegung versetzt wird. Man bestimmt während der konstanten Rotation diese Momente, indem man durch den Reizkontakt $p d$ und den Schließkontakt $p_{1} Q Q p_{2}$ den Strom eines Elementes zum Galvanometer leitet und diejenige Stellung des Schiebers an der Teilung abliest, bei welcher die Ablenkung eben erscheint und bei der sie aufhört. Hat man diese Punkte bestimmt, aus denen man auch die Schließungszeit erhält, so kann man sich überzeugen, daß dieselben am Schluß einer Beobachtungsreihe mit ausreichender Genauigkeit dieselben geblieben sind 1). Auch sind die sc erhaltenen Ablenkungen während der Rotation durchaus konstant.

Fig. 12 gibt die Anordnung eines solchen Versuches schematisch wieder. Vom Längs - und Querschnitt $l q$ ist ein langer Nerv (z. B. Nerv. ischiad. des Frosches) oder langer Muskel (z. B. M. sartorius) zum Galvanometer $M$ durch den Schließkontakt

1) Ich hebe diesen Umstand besonder's hervor, da L. Hermann sich veranlaßt sah, wegen angeblich unsicheren Kontaktes im Quecksilber stattdessen harte Schleifkontakte zwischen Kupferbänken und Drahtbürsten anzuwenden. 
$p_{1} Q Q p_{2}$ periodisch abgeleitet. Auch diese Ablenkung muß bei gut eingestelltem liontakt eine konstant bleibende sein, aber es ist zweckmäßig, den Nerven- oder Muskelstrom mit Hilfe eines Rheochordstromes (s. oben S. 7) zu kompensieren, damit die Nerven und Muskeln nicht durch ihre Eigenströme gereizt werden. Am anderen Ende des Nerven oder Muskels befinden sich die Reizelektroden $r r$, welche unter Vorlegung eines Kurzschlüssels $L$ mit der sekundären Spule $S$ eines Induktoriums verbunden sind.

Fig. 12.

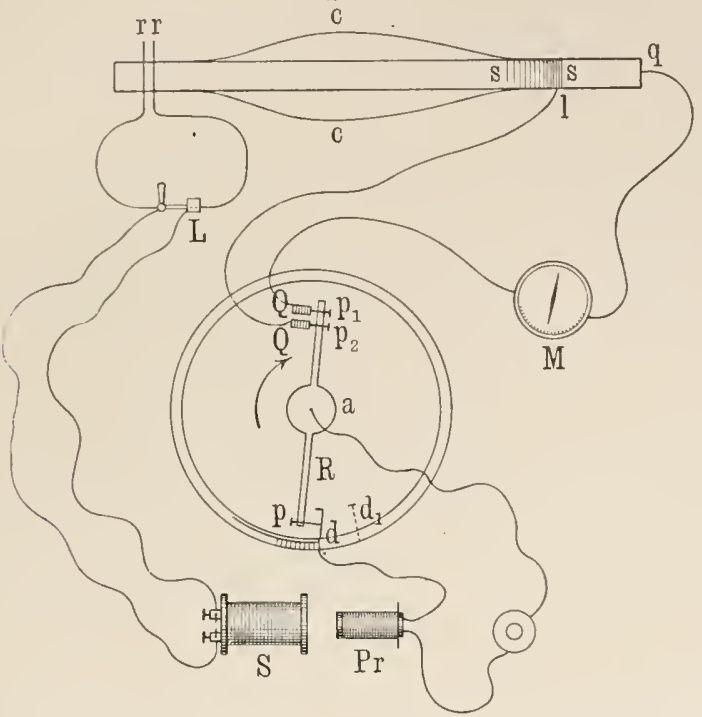

Anordnung eines Rheotomversuches. ss Reizwelle, c c Kontraktionswelle.

Der Strom der primären Spule $P r$ wird durch den Reizkontakt $p d$ geleitet, indem der Strom in der Achse $a$ zu einer kreisförmigen Quecksilberrinne geht, in welche ein Draht $R$ eintaucht, der zur Spitze $p$ führt.

Der Versuch wird in der Weise ausgeführt, daß man den Schieber mit dem Reizkontakt $p d$ zuerst auf den Moment der Öffnung des Schließkontaktes $p_{1} Q Q p_{2}$ einstellt, wie es die Fig. 12 angibt. Dies ist der an der Teilung abgelesene Nullpunkt. Man reizt, indem man den Kurzschlüssel $L$ öfnet. Ist das Rheotom auf den Nullpunkt eingestellt, so muß das Rad desselben nahezu 
eine ganze Umdrehung machen, bis wieder eine Schließung des Galvanometerkreises erfolgt. In dieser Zeit, $1 / 5$ bis $1 / 10^{\prime \prime}$, ist im Nerven und meist auch im Muskel (abgeseheu von schwachen Nachwirkungen, s. unten) der ganze Prozeß der Stromesschwankungen abgelaufen. In solchem Falle tritt daher keine Änderung am Galvanometer ein. Verschiebt man aber den Reizkontakt in der Richtung nach $d_{1}$, so vergeht zwischen Reizmoment und darauf folgender Öffnung des Galvanometerkreises eine bestimmte Zeit, und wenn in dieser sich die Reizwelle bis zur abgeleiteten Stelle $l_{q}$ fortgepflanzt hat, so wird am Galvanometer eine Ablenkung im Sinne einer negativen Schwankung eintreten. Verscbiebt man daher den lieizkontakt allmählich in dieser

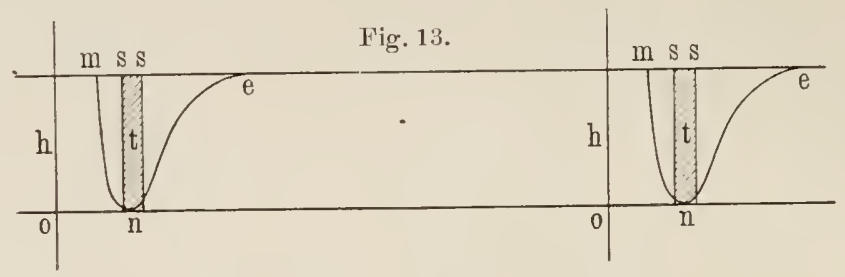

Zeitlicher Verlauf der negativeu Schwankung.

Richtung, so findet man ein Zeitintervall, nach welchem die negative Schwankung eben merklich beginnt, bei weiterer Verschiebung zu einem Maximum anwächst und dann bei fortgesetzter Verschiebung des Reizkontaktes weiter auf nahezu Null absinkt. Je kleiner die Schließungszeit ist, desto genauer werden die erfolgenden Ablenkungen die ganze Kurve der Reizwelle wiedergeben, welche über den Längsschnittpunkt 7 abläuft. Fig. 13 gibt den zeitlichen Ablauf zweier aufeinander folgender Schwankungen $m n e$ des Rubestromes von der Höhe $h$ auf der Zeitabszisse 00 aufgetragen wieder. Die Zeitpunkte 0 sind die Momente der Reizung. Die Zeit vom Momente 0 bis zum Beginn $m$ der negativen Schwankung ist die Zeit der Fortpflanzung der Reizwelle von der gereizten Stelle $r r$ bis zum abgeleiteten Längsschnittpunkte 1. Die hieraus berechnete Geschwindigkeit der Reizwelle stimmt, wie schon oben bemerkt, mit der durch Muskelzuckung gemessenen Geschwindigkeit der Erregung vollständig überein. Man erkennt an der negativen Schwankung aber auch den ganzen Ablauf des Erregungsprozesses. Stellen wir die mit der elektrischen Ver- 
änderung zusammenfallende Zustandsänderung als Kurve dar, die wir in einem bestimmten Zeitpunkte nur auf die Länge eines Nerven oder Muskels aufgetragen denken, so erhalten wir die Form der Reizwelle, wie sie über die Fasern abläuft. In Fig. 13 ist das Kheotom so eingestellt gedacht, daß die Schließungszeit, zwischen $s s$ fallend, mit dem Maximum der Schwankung zusammenfällt. Das auf das Galvanometer wirkende Stromintegral $(h . t)$ ist als schraffiertes Flächenstäck gezeichnet. Denken wir uns dieses statt des Reizkontaktes über die ganze Kurve verschoben, so erhalten wir die verschiedenen Ablenkungen des Versuches ${ }^{1}$ ). Man erkennt aus den Figuren, daß der aufsteigende Teil der Reizwelle bis zum Maximum steiler ist, als der absinkende. Man sieht leicht ein, daß man aus diesen Messungen nicht nur die Geschwindigkeit der Reizwelle erhalten kann, sondern auch ihre Dauer. Diese 1)auer ergibt uns also einen Aufschluß über die Schnelligkeit, mit welcher der Erregungsprozeß in den Organen nach einem Momentenreiz steigt, wio lange er andauert und wie geschwind er wieder in den Ruhezustand übergeht.

Bisher ist immer nur von der negativen Schwankung des Längsquerschnittstromes die Rede gewesen. Aber schon du BoisReymond hat an Nerven untersucht, wie sich der Vorgang bei der Reizung verhält, wenn man ihn von zwei Punkten seines Längsschnittes ableitet. Er fand, daß bei Ableitung zweier zum Äquator symmetrischer Stellen (s. oben S. 6 u. 15), welche keinen Ruhestrom geben, auch bei der Reizung keine Wirkung am Galvanometer auftritt, daß dagegen andere Stellen, welche mehr oder weniger elektromotorisch wirken, auch eine der Größe des Ruhestromes entsprechende negative Schwankung geben. Spätere Versuche, welche ich am unverletzten langen, parallelfaserigen und kuraresierten Muskel anstellte, ergaben ein ähnliches, wenn auch, wie man bald einsehen wird, nicht ganz gleiches Resultat. Versuche, welche ich mit Hilfe des Rheotoms zuerst an solchen Muskeln ausführte, zeigten, daß bei Ableitung zweier Längsschuittpunkte,

$\left.{ }^{1}\right)$ Es wurde an dem Rheotom der Reizkontakt beweglich, der Schließkontakt dagegen fest angebracht, weil die Verschiebung des letzteren merkliche Änderungen der Schließungszeit durch kleine Abweichungen der Grundplatte des Rhentoms von der Horizontalebene herbeiführen würde. 
welche keinen oder einen möglichst schwachen Strom geben und weit genug voneinander entfernt waren, zwei kurzdauernde einander entgegengesetzte Ströme entstehen, welche bei dauerndem Galvanometerschluß sich ganz oder teilweise aufheben. In Fig. 14 ist der Vorgang der sich fortpflanzenden Reizwelle des in $r r$ gereizten Muskels dargestellt und die damit verbundenen Stromschwankungen. Sind die beiden Stellen $l_{1}$ und $l_{2}$ durch das Rheotom zum Galvanometer geleitet, so beobachtet man zuerst einen kurz dauernden Strom in der Richtung der Pfeile 1 und dann schnell darauf einen solchen in der Richtung der Pfeile 2.

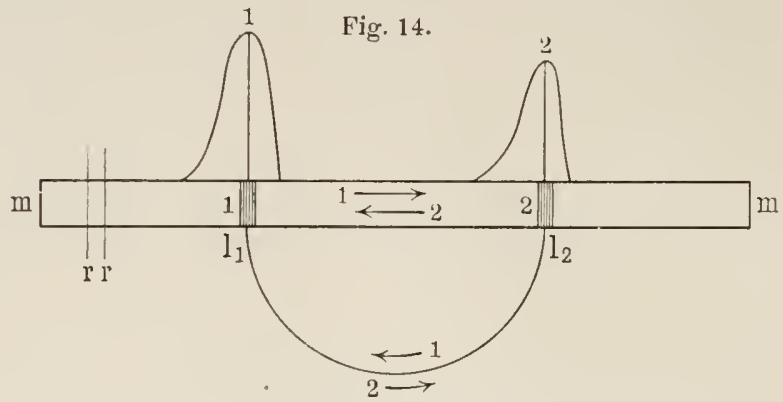

Fortpflanzung der Reizwelle.

Das heißt, zuerst wird die Stelle $l_{1}$ negativ gegen die Stelle $l_{2}$, und dann die Stelle $l_{2}$ negativ gegen die Stelle $l_{1}$. Die Versuche ergeben also, wie die Figur es darstellt, daß jede Stelle des Muskels, welche sich innerhalb der fortschreitenden Reizwelle, also in Erregung befindet, sich negativ gegen eine ruhende Stelle verhält. Man kann diesen Vorgang auf die negative Schwankung zweier entgegengesetzt gerichteter Längsquerschnittströme zurückführen, indem man sich die beiden Muskelelemente 1 und 2 abgeleitet denkt. Wenn die Reizwelle in 1 anlangt, tritt negative Schwankung des Elementes 1 ein, und daher überwiegt der Ruhestrom von Element 2 in der Richtung des Pfeiles 1, wenn die Reizwelle aber über Element 2 steht, so erleidet dieses negative Schwankung und es überwiegt der Ruhestrom des Elementes 1 in der Richtung des Pfeiles 2. Es treten also zwei Phasen einer Stromschwankung ein. Man hat diese Ströme nach Hermainn anch die doppelphasigen Aktions- 
ströme genaunt, und diese Bezeichnung auch auf die negative Schwankung des Längsquerschnittstromes angewendet, den man als einphasigen Aktionsstrom bezeichnet hat. Es ist aber einleuchtend, daß beide Vorgänge auf denselben Ursachen beruhen, und daß die negative Schwankung des Längsquerschnittstromes der einfachere Vorgang ist, auf welchen die doppelphasigen Aktionsströme eines unverletzten Muskels zurückgeführt werden müssen. Was die Messung des Ablaufes dieser Ströme anbetrifft, so kann nur die der negativen Schwankung des Längsquerschnittstromes (also des einphasigen Aktionsstromes) ein zuverlässiges Bild geben, weil bei Ableitung eines unverletzten Muskels die beideu Ableitungsstellen oft einander so nahe liegen, daß die beiden einander entgegengesetzten Phasen mehr oder weniger miteinander interferieren werden. Auch bei Ableitung eines unverletzten Muskels von Längsschnitt und Sehne, der einen schwachen oder keinen Ruhestrom liefert, z. B. des Musculus gastrocn. (Frosch), erhält man auf Nervenreizung einen doppelphasigen Aktionsstrom, indem zuerst der Längsschnitt gegen die Sehne und dann die Sehne gegen den Längsschnitt negativ wird ${ }^{1}$ ). Dies erklärt sich sebr einfach daraus, daß die Nervenfasern etwa in der Mitte der Muskelfasern eintreten, hier demnach die Rieizwelle anhebt und sich nach dem Achillessehnenende fortpflanzt. Da aber die Fasern dieses Mruskels sehr kurz sind, so ist die erste Phase noch nicht beendet, wenn die zweite bereits anfängt. Man ersieht hieraus, daß man an unregelmäßig gestalteten, unverletzten Muskeln, bei denen die Nerveneintrittsstellen nicht selten über weite Strecken verteilt sind, keine eindeutigen Resultate erhalten wird und gewisse prinzipielle Fragen an ihnen nicht lösen kann. Aus diesem Grunde habe ich mich bei weiteren Untersuchungen und Messungen eines möglichst langfaserigen regelmäBigen, kuraresierten II uskels bedient, der an einem Ende direkt gereizt wurde. Am Muskel ergab sich die für die Theorie wichtige Tatsache, daß die negative Schwankung selbst bei der stärksten Reizung des Längsquerschnittstromes im Maximum nur bis Null sinken kann, ihn aber nicht umkehrt, wie es die Kurve $m n e$ in Fig. 87 zeigt.

Wio am Nerven läßt sich auch am Muskel die Geschwindigkeit messen, mit welcher sich die Reizwelle fortpflanzt, ebenso ihr

1) Sigm. Ma a er, Archiv f. Anatomie n. Physiol. 1868, S. 655. 
ganzer Ablauf und ihre Dauer. Hierbei tritt uns die wichtige Frage entgegen, wie sich der elektrische Prozeß zur Kontraktion des Muskels verhält. Schon aus älteren Versuchen von $\mathrm{Helm-}$ holtz über die Zeitmomente der primären und sekundären Zuckung kounte man schließen, daß die negative Schwankung schneller auftritt als die Zuckung des primären Muskels. Man weiß aus den $\mathrm{Helmholtzschen} \mathrm{Untersuchungen} \mathrm{über} \mathrm{den} \mathrm{zeit-}$ lichen Verlauf der Muskelzuckung, die mit Hilfe der Pouilletschen Zeitmessungsmethode oder mit dem Myographion angestellt wurden, daß die Zuckung nicht sofort im Momente des Reizes beginnt, sondern erst nach einem "Stadium der latenten Reizung", das im Froschmuskel unter gewöhnlichen Bedingungen der Arbeitsleistung etwa bis zu 0,01" reicht ${ }^{1}$ ). Die ganze Zuckung dauert beim Froschmuskel etwa 1/5". Weitere Versuche, welche vou J. Bernstein (I. c.) angestellt wurden, ergaben an langen, parallelfaserigen und kuraresierten Muskeln, daß bei Reizung an einem Ende (wie in Fig. 11) die sich über den Muskel fortpflanzende Kontraktion eine Geschwindigkeit vou etwa $3 \mathrm{~m}$ in der Sekunde besitzt. Da sie an jeder Stelle demnach in verschiedenen Zeitpunkten anhebt, das Maximum erreicht und wieder sinkt, so läuft sie als Kontraktionswelle über die ganze Länge der Fasern ab. In der Fig. 12 ist durch die Kurven $c c$ die Verdickung eines sehr lang gedachten Muskels (abgesehen von der gleichzeitigen Verkürzung) dargestellt, und zwar für den Zeitmoment, in welchem die schraffiert angegebene Reizwelle $s s$ eben den Längsschnittpunkt $l$ erreicht ${ }^{2}$ ). An der Reizstelle $r r$ entstehen beide durch den Induktionsschlag, die Reizwelle momentan obne wirkliches Latenzstadium, die Kontraktionswelle aber erst nach etwa "1/100". Die Reizwelle pflanzt sich nun mit derselben Geschwindigkeit von $3 \mathrm{~m}$ in $1^{\prime \prime}$ fort wie die Kontraktionswelle; sie eilt daher der Kontraktionswelle voraus und muß in jedem Querschnittelement der Muskelfaser früher erscheinen als der Beginn der Kontraktion. Der größte Teil

1) Mit feineren Hilfsmitteln photographischer Aufzeichnung beobachtet, kann dieser Zeitraum bis auf etwa 0,004" reduziert werden.

") Das schematische Bild gibt die wirklichen Verhältnisse der Wellenlänge nicht genau wieder. Die Kontraktionswelle ist sehr viel länger als die Reizwelle. Ihre Länge beträgt im Froschmuskel etwa $300 \mathrm{~mm}$. 
der Reizwelle (negative Schwankung, Phase) in jedem einzeluen Muskelelement vollzieht sich daher im Stadium der lateuten Reizung.

Man wird aus dieser. Tatsache schließen dürfen, daß der gesamte Erregungsprozeß in dem Muskel, welcher die Kontraktion bedingt, kein einbeitlicher ist, da die elektrischen und mechanischen Yeränderungen desselben zeitlich nicht zusammenfallen. Beiden Vorgängen, den elektrischen wie mechanischen, liegen unbedingt chemische Prozesse zugrunde, die in der Muskelsubstanz ablaufen. Wir wissen mit Bestimmtheit, daß bei der Muskeltätigkeit eine stärkere Spaltung und Oxydation von organischen Verbindungen in dem Muskel eintritt, als dies in der Ruhe geschieht, und daß der Muskel bei der Tätigkeit mehr Sauerstoff verbraucht und unter den Verbrennungsprodukten hauptsächlich mehr Kohlensäure liefert als in der Ruhe. Auch die Wärmeerzeugung in IIuskel, die bei der Arbeitsleistung eintritt, ist ein Beweis dafür, daß bei der Reizung chemische Energie umgesetzt wird. Wir werden daher sagen dürfen, daß der gesamte chemische Prozeß bei der Kontraktion in zwei Teilprozesse zerfällt. Der erste fällt mit der elektrischen Veränderung, der zweite mit der mechanischen Veränderung der Muskelfaser zeitlich zusammen. Der erste fällt zum großen Teil in das Stadium der Latenz und muß bis zu einem gewissen Grade vorgeschritten sein, damit der zweite zugleich mit der Kontraktion erfolgen kann. Welcher Art der erste Teilprozeß ist, möge zunächst unbestimmt bleiben; daß der zweite im wesentlichen in einer oxydativen Spaltung organischer Verbindungen besteht, kann wohl als sicher angesehen werden. Weiteres hierüber wollen wir später behandeln.

Diese elektrischen Veränderungen der Muskeln und Nerven in ihrer Beziehung zum Ablauf der Erregung und Tätigkeit sind uun in den letzten Jahrzehnten auch mit Hilfe von elektrischen Instrumenten beobachtet worden, welche schnellen Stromesschwanknngen mit großer Schnelligkeit folgen und daher die Anwendung des Rheotoms zum Teil ersetzen können. Zu diesen gehört erstens das von dem Physiker Lippmann erfundene Kapillarelektrometer und zweitens das ron dem Physiologen Einthoven konstruierte Saitengalvanometer ${ }^{1}$ ). Beide Instru-

1) Buschreibung dieser Instrumente siehe im Anhang. 
mente folgen schnellen Stromesoszillationen bis zu einer gewissen Grenze, und man kann nach bekannten Methoden diese Bewegungen auf einem rotierenden Zylinder photographisch verzeichnen. Man darf aber bei solchen Untersuchungen niemals außer acht lassen, daß die erhaltenen photographischen Kurveu keineswegs bei schnelleren Schwankungen die wirklichen Stromeskurven sind, und daß man diese erst berechnen muß, soweit es möglich ist. Nach dieser Methode hat man im allgemeinen die mit dem Rheotom erhaltenen Resultate bestätigt. Eine große Zahl von Versuchen sind von verschiedenen Beobachtern über den Ablauf der Aktionsströme besonders an unverletzten Muskeln gemacht worden. Da hat man an einigen Muskeln, wie am M.gastrocn. des Frosches, für den doppelphasigen Aktionsstrom bei Nervenreizung eine photographische Kurve erhalten, an noch unregelmäßiger gestalteten Muskeln, wie z. B. am Triceps femoris oder Gracilis, kann man noch kompliziertere Kurven, auch dreiphasige erhalten. Aber es ist klar, daß solche Versuche gar keinen prinzipiellen Wert haben können, wenn es sich um die Fragen handelt: wie lange danert an jeder Stelle einer Muskelfaser die elektrische Reizwelle, und in welchem Zeitverhältnis steht sie zur Kiontraktionswelle? Es ist klar, daß man au unregelmäßig gestalteten Muskeln, noch dazu an solchen, mit mehreren Sehnen, wie der Triceps femoris, oder Zwischensehnen, wie der M. gracilis, deren Muskelfasern die verschiedensten Längen haben und an denen die Eintrittsstellen der Nervenfasern sich über weite und voneinander getrennte Strecken der Muskeln verbreiten, keine klaren und maßgebenden Resultate erhalten kann, und daß diese auch je nach der Art der Ableitung mannigfach schwanken müssen. Wenn man an zwei Stellen eines unverletzten Muskels ableitet, so ist ferner zu berücksichtigen, daß, wenn die Ableitungsstellen einander nahe liegen, die beiden Phasen der Aktionsströme mehr oder weniger miteinander interferieren müssen. Es wird daher ein klares und eindeutiges Resultat nur erhalten werden, wenn man sich erstens eines möglichst langen parallelfaserigen Muskels bedient, und wenn man zweitens zurVermeidung aller Interferenzen an einem Ende des Muskels die negative Schwankung des Längsquerschnittstromes, d. h. des einphasigen Aktionsstromes verzeichnet. Soll nun mit dem Ablanf dieses Stromes der Ablauf der Kontraktionswelle verglichen werden, so darf man nicht, wie es meist geschehen ist, 
die Zuckungskurve der Verkürzıng des ganzen Muskels gleichzeitig myographisch aufnehmen, sondern nur den Ablauf der Kontraktion an der abgeleiteten Längsschnittstelle; denn wir wollen ja durch den Versuch feststellen, in welchen Zeitmomenten die Reizwelle und die Kontraktionswelle von der Reizstelle des Muskels aus an dem abgeleiteten Längsschnittpunkte anlangt, wann beide Wellen an dieser Stelle ihren Höhepunkt erreichen und wann sie daselbst abgelaufen sind. Wenn wir aber die Verkürzungskurve des ganzen Muskels aufzeichnen, so ist einleuchtend, daß diese in allen ihren Teilen früher beginnen muß als die liontraktionswelle am abgeleiteten Längsschnittpunkt, da sie mit der Kontraktion der gereizten Stelle zugleich anhebt. Infolge der Vernachlässigung dieses Umstandes hatte man noch dazu an dem unregelmäßig gebauten M. gastrocn. Resultate erhalten, welche mit denen der Rheotomversuche niclit stimmten und in denen die Alktionsströme weit in den Verlauf der Muskelzuckung hineinreichten.

Von Bernstein und Tschermak ${ }^{1}$ ) wurde daher die Kapillarelektrometerkurve der negativen Schwankung des Längsquerschnittstromes und die Kontraktionswelle der abgeleiteten Längsschnittstelle an einem langen parallelfaserigen Muskel (M. adductor des Frosches) in folgender Weise aufgenommen. Man denke sich diesen Muskel, wie in Fig. 11, von $l$ und $q$ abgeleitet, horizontal auf einer festen Unterlage gelagert. Anstatt zum Rheotom führt die Leitung von $l$ und $q$ zum Kapillarelektrometer. Über die abgeleitete Längsschnittstelle $l$ ist ein schmales durchfeuchtetes Leinwandband gelegt, welches unten an einen leichten, wenig belasteten Hebel angreift, der bei der Verdickung des Muskels an dieser Stelle sich hebt und daher auf einem rotierenden Zylinder die Verdickungskurve der Stelle l, d. h. den Ablauf der Kontraktionswelle über die Stelle 7 zeichnen würde. Diese Zeichnung geschieht nun in diesem Falle photographisch dadurch, daß der Hebel an den Spalt gestellt wird, auf welchen das Bild der Kapillare entworfen ist. Gehen die Lichtstrahlen durch diesen Spalt hindurch, so zeichuen sie auf der photographischen Platte den Schatten des

1) Über die Beziehung der negativen Schwankung des Muskelstromes zur Arbeitsleistung des Muskels. Ptlügers Arch. f. d. ges. Physiol. 89, 289-331 (1902). 
deckenden Hebelpunktes auf, der, wenn er sich hebt, bei der Bewegung der Platte die Kurve der Kontraktionswelle zeichnet. Ebenso zeichnet der Meniskus der Kapillare bei derselben Reizung die Elektrometerkurve der Reizwelle. Die Bedingungen des Versuches fallen nun mit denen des Rheotomversuches zusammen, und es hat sich auch eine befriedigende Übereinstimmung ergeben.

In Fig. 15 ist das photographische Bild (ein l'ositiv des Originalnegativs) eines solchen Versuches am Musc. adductor femoris des Frosches wiedergegeben. Der Muskel ist etwa in seiner Nitte vom Längsschnitt und am unteren Ende vom künstlichen

Fig. 15.

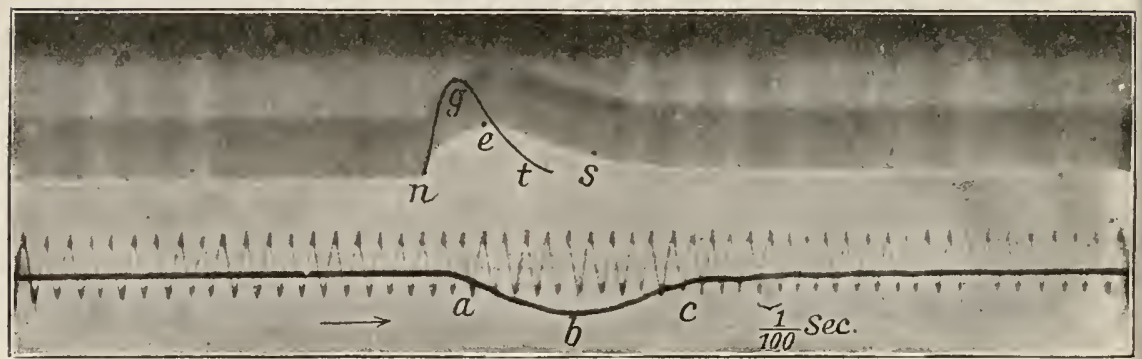

Reizwelle und Kontraktionswelle des IIuskels.

$n$ es Kapillarelektrometerkurve, $\boldsymbol{n} g \boldsymbol{t}$ berechuete Kurve der negativen Schwankung (schematisch), abc Kontraktionswelle.

(thermischen) Querschnitt abgeleitet, der Ruhestrom ist kompensiert (s. oben S.7). Die Bewegung des Meniskus infolge einer negativen Schwankung bei Einzelreiz (Öffnungsinduktionsschlag) ist, im Bilde nach oben gerichtet, durch die Kurve nes angegeben, welche von links nach rechts zu lesen ist. Sie entspricht der über die abgeleitete Stelle sich fortpflanzenden Reizwelle. Der Ablauf der Kontraktionswelle (Verdickungskurve) an der abgeleiteten Längsschnittstelle wird durch die Kurve $a b c$ gegeben, welche der Schatten des Myographionhebels vor dem Spalt zeichnet (im Projektionsbilde sind alle Bewegungen, welche in Wirklichkeit nach unten gerichtet waren, nach oben gewendet). Die Schwingungen einer vor dem Spalt schwingenden, horizontal liegenden Federlamelle (elektromagnetisch durch einen akustischen Unterbrecher in Bewegung erhalten) geben die Zeit an; jede ganze Periode bedeutet $1 / 100$ Sek. Nan erkennt deutlich, daß die Elektro- 
meterkurve nes der Zuckungskurve abc vorausgeht. Der Beginn der Elektrometerkurve liegt ungefähr ${ }^{1}{ }_{100}$ Sek. vor dem in diesem Falle merklichen Beginn der Zuckungskurve. Der Gipfel der ersteren liegt weit vor dem der letzteren, und ebenso verhalten sich die allmählicher ablaufenden Enden beider lí urven zueinander. Die Elektrometerkurve nes ist aber keineswegs die wirkliche liurve der negativen Schwankung, vielmehr muß dieselbe durch Rechnung (s. S. 50 und Anhaug) gefunden werden; dieselbe ist auf Grund vou Berechnungen spezieller Versuche schematisiert in die Figur als Kurve $n g t$ eingetrageu. Nun erkennt man mit großer Deutlichkeit, daß der Gipfel $g$ der negativen Schwankung schou erreicht ist, bevor die Kontraktionswelle an der abgeleiteten Stelle merklich anhebt. Der aufsteigende Teil der Reizwelle liegt für jedes Muskelelement zum allergrößten Teil innerhalb des Stadiums der latenten Reizung. Der abfallende Teil der lieizwelle reicht zeitlich mehr oder weniger weit in den Ablauf der liontraktionswelle hinein, sinkt viel langsamer ab als der aufsteigende und hat kein scharf zu bestimmendes Ende. Meist ist im Maximum der Kontraktion die lieizwelle schon stark abgesunken. Die Gestalt der Reizwelle ist aus der Figur der Kurve ngt ersichtlich. Sie steigt schnell konvex nach obeu gerichtet zum Maximum auf, sinkt zuerst schnell nach oben konvex ab und dann durch einen Wendepunkt gehend konkar nach oben gerichtet allmählich zur Abszisse ab. Nimmt man für die [)auer der Kontraktionswelle nach Fig. 15 etwa 0,12 Sek. an, so würde, abgesehen von dem allıählich ablaufenden Ende, die Dauer der ganzen Reizwelle auf etwa 0,06 Sek. berechnet werden können. Es unterliegt wohl keinem Zweifel, daß in dem vom lebenden Körper getrennten, nicht mehr normal er'nährten uud durch einen Querschnitt verletzten Muskel die Schnelligkeit aller Erregungsprozesse verlangsamt sein muß. Die Dauer der Reizwelle wird also unter ganz normalen Bedingungen wohl eine viel geringere sein, als man sie in dem Experiment an herausgeschnittenen verletzten Muskel findet ${ }^{1}$ ). Es läßt sich aus vielen Beobachtungen schließen, daß im absterbenden Muskel eine viel längere Nachwirkung jeder

1) In den oben beschriebenen Rheotomversuchen konnte nur der höhere Teil der Reizwelle gemessen werden, der etwa 0,004" betrug, da bei repetierender 5 - bis 10 maliger Reizung in der Sekunde eine Summierung der abfallenden Teile der Welle eintritt. 
Reizung eintritt als im normalen, was sich an der schnelleren Ermüdung bemerkbar macht. Der Verlauf der Reizwelle und der Kontraktionswelle ist daher ein gutes Zeichen für den Zustand des Muskels. Je besser der Muskel ernährt wird, um so schneller kehrt er nach jeder Reizung in den normalen Ruhezustand wieder zurück, und dies erkennt man an der schneller ablaufenden Reizwelle und Kontraktionswelle. Daf die Reizwelle in einem normal er'nährten, unverletzten Muskel, innerhalb des lebenden Körpers, eine viel geringere Dauer besitzt, daß namentlich der abfallende Teil viel schneller auf Null sinkt als in dem obigen Falle, dafür sprechen mancherlei Beobachtungen. Erstens kann man auch an dem unverletzten Muskel einen schnelleren Ablauf der beiden Phasen des Aktionsstromes beobachten und dann scheinen auch innerhalb des lebenden Körpers diese Vorgänge noch schneller und in kürzerer Zeit abzulaufen. Aber es ist klar, daßs solche Messungen nur dann ein genaueres Resultat geben können, wenn die beiden Ableitungsstellen der Muskeln so weit voneinander entfernt liegen, daß die Reizwelle an der ersten Stelle, die sie passiert, schon vollständig abgelaufen ist, bevor sie die zweite erreicht. Das ist aber bei den kurzen Froschmuskeln, die meist zu solchen Versuchen verwendet worden sind, durchaus nicht der Fall, auch wenn wir die längsten Muskeln dazu auswählen. Dazu kommt noch, daß Versuche, in denen die Reizung rom Nerven aus geschieht, für eine genauere Messung untauglich sind, da in deu meisten Muskeln die Fasern sich über ein so großes Gebiet des Muskels erstrecken, daß die Reizwellen in den verschiedenen Muskelfasern an weit voneinander entfernten Stellen anheben und daher keineswegs zu gleicher Zeit an den Elektrodenstellen anlangen. Es ist schon aus den ersten Rheotomversuchen von mir der Schluß gezogen worden, daß bei NervenJeizung in jeder Muskelfaser die Reizwelle an der Eintrittsstelle der Nervenfaser beginnen muß, um sich von dort nach beiden Seiten hin ebenso wie die Kontraktionswelle fortzupflanzen. Das, was wir also in diesem Falle beobachten, ist nichts auderes als eine liombination von Aktionsströmen, wolche in verschiedenen Fasern ungleichzeitig erfolgeı, sicls zum Teil summieren oder auch subtrabieren können. Von diesem Gesichtspunkte aus habe ich in meinen ersten Rheotomversuchen es vorgezogen, die prinzipiellen Fragen, $1 m$ die es sich zunächst handelte, nicht mit 
Anwendung der Nervenreizung, sondern durch direkte Muskelreizung, und zwar nm alle Nervenwirkung auszuschließen, an kuraresierten Muskeln anzustellen. Von diesem Gesichtspunkte aus muß man auch alle Resultate beurteilen, welche später mit dem Rheotom und in neuerer Zeit mit dem Kapillarelektrometer und Saitengalvanometer bei Reizung vom Nerven aus erhalten worden sind. Da findet man daun meist, selbst an dem kurzfaserigen Musc. gastrocn. vom Frosch, einen doppelphasigen Aktionsstrom Fig. 16.

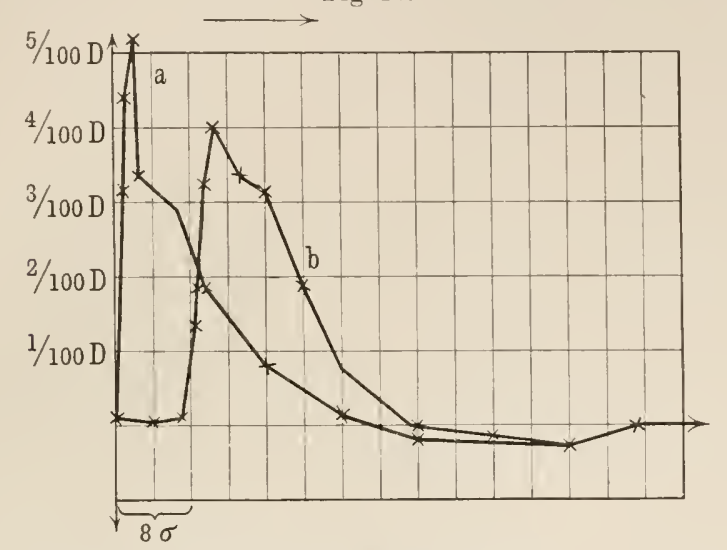

Einphasiger Aktionsstrom des Musculus sartorius rom Frosch.

« Ableitung von der Reizstelle,

$b$ Ableitung von einem $20 \mathrm{~mm}$ entfernten Puukt (nach Garten).

bei Nervenreizung vor. Aus Rheotomversuchen am Musc. gastrocn. des Frosches vom Jahre 1867 bei Reizung seines Nerven (35 mm Reizstelle vom Muskel entfernt) konnte ich folgende Werte erhalten $\left.{ }^{1}\right)$. In einer Zeit von $5 \sigma\left(1 \sigma={ }^{1} /{ }_{1000}\right.$ Sek.) begann die erste Phase. Diese Zeit wird von der Fortpflanzung der Nervenerregung bis zu den Nervenendigungen im Muskel eingenommen, und binzuaddiert sich noch eine Erregungszeit der Nervenendorgane von etwa ${ }_{300}$ Sek. Diese Phase erreichte in etwa 2,5 $\sigma$ rom Beginn ihr Maximum, dann trat im Zeitpunkt 6,5 $\sigma$ die zweite, entgegengesetzte Phase auf und erreichte zur Zeit 7,5 $\sigma$ bereits ihr Maximum, um dann langsamer abzufallen.

$\left.{ }^{1}\right)$ Die Erregungszeit der Nervenendorgane in den Muskeln. du Bois' Arch. 1882, S. 344. 
Ähnliche Werte 1,1 bis 1,5 б für die Anstiegszeit der ersten Phase am direkt gereizten Froschmuskel fand L. Hermann mit Hilfe eines Fallrheotoms ${ }^{1}$ ). Ebenso fand Garten ${ }^{2}$ ) hierfür 1,6

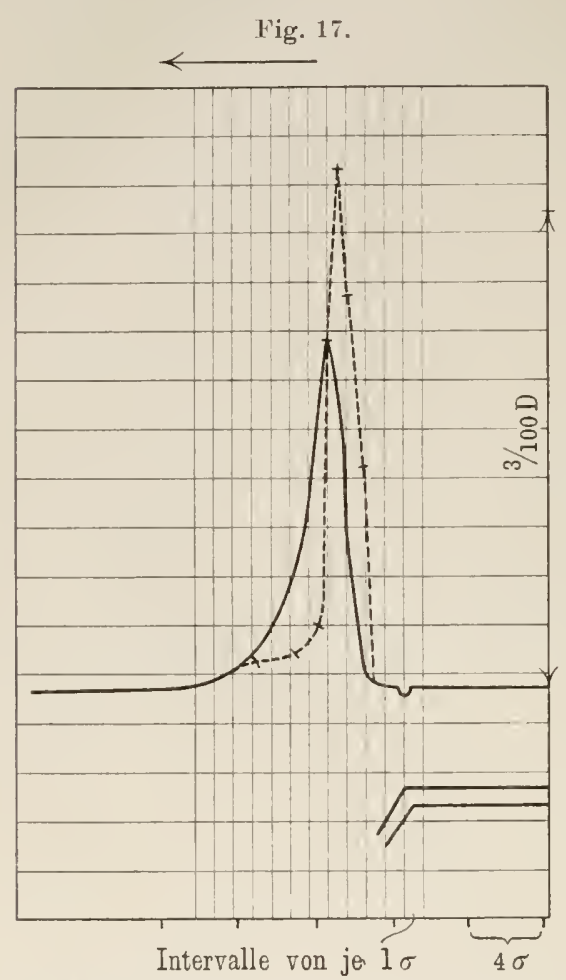

Einphasiger Aktionsstrom (negative Schwankung) vom Musculus gastroenemius des Kaninchens. Die ausgezogene Linie stellt die gezeichnete, die gestrichelte die berechnete Kurve dar (nach Garten, Beiträge zur Kenntnis des Erregungsvorganges der Nerven und Muskel des Warmblüters, Zeitschr. f. Biolog. 52, 1909). bis 2,0 $\sigma$ an dem einphasigen Aktionsstrom des Musc. sartorius des Frosches mit Hilfe des Kapillarelektrometers an der direkt gereizten Stelle. Mit der Fortpflanzung auf $20 \mathrm{~mm}$ nahm diese Zeit auf 2,4 bis $3,2 \sigma \mathrm{zu}$. Hiernach scheint die Reizwelle bei der Fortpflanzung ihre Gestalt zu ändern, wie es die von Garten angegebene Fig. 16 zeigt.

Mit Hilfe des Saitengalvanometers hat Garten ferner am Musc. gastroen: des lebenden Kaninchens den ein phasigen Aktionsstrom (negative Schwankung des Längsquerschnittstromes) bei Rieizung vom Nerven aus aufgenommen, wie Fig. 17 zeigt. Die gestrichelte Kurve zeigt in rober Annäherung (leider ist eine genauere Analyse der Saitengalvanometerkurven noch nicht gefunden) den wirklichen Verlauf des Stromes. Die Anstiegszeit beträgt etwa $2 \sigma$, und der Abfall nimmt, abgesehen

1) Pflügers Arch. 15, 233 (1877).

2) Ber. d. Sächs. Ges. d. Wiss. 26, 1901. 
von dem allmählich ablaufenden Ende, etwa dieselbe Zeit in Inspruch. Die ganze Dauer des Hauptteiles zu 4 bis $5 \sigma$ angenommen, ist also anßerordentlich kurz.

Das letztere Resultat bestätigt den schon aus meinen älteren Versuchen aufgestellten Satz, der viel bestritten worden ist, dab die Reizwelle (negative Schwankung = einphasiger Aktionsstrom) an jeder Muskelstelle in ihrem Hauptteile bereits abgelaufen ist, bevor eine merkliche Zusammenziehung eintritt; denn die latente Reizung an jeder Muskelstelle ist mindestens zu $4 \sigma$ anzunehmen. Bis jetzt sind leider Versuche, in denen Reizwelle (Aktionsstrom) und Kontraktionswelle von derselben Muskelstelle verzeichnet wurden (wie oben), an lebenden Warmblütern noch nicht angestellt worden.

Auch an Muskeln des lebenden Menschen hat man die doppelphasigen Aktionsströme nachweisen können. L. Hermann. beobachtete (1878) mit Hilfe des Rheotoms bei Reizung des Nerv. medianus am ()berarm an den Bengemuskeln der Hand und der Finger am Unterarm bei Ableitung von zwei Stellen der Haut daselbst einen doppelphasigen Strom, der hauptsächlich von den genannten Muskeln herrühren mußte. In neuerer Zeit haben Piper ${ }^{1}$ ) und Garten ${ }^{2}$ ) solche Versuche mit Hilfe des Saitengalvanometers angestellt und bei genügender Entfernung der beiden Ableitungsstellen voneinander meist einen doppelphasigen Strom erhalten, wie Fig. 18 zeigt. Es ist klar, daß man von verschieden gelegenen Ableitungsstellen verschiedene Kurven erhalten wird, daß aber auch bei weit voneinander gelegenen Ableitungen keine einfachen Resultate, welche zur Messung des Verlaufes geeignet wären, erscheinen können, da die betreffenden Muskeln sehr unregelmäbig gestaltet sind, ihre Muskelfasern sehr verschiedene Länge besitzen und die Ausbreitungs- und Eintrittsstellen der Nervenfasern sich über weite Strecken ausdebnen. Daraus erklärt es sich wohl, daß die Anstiegszeiten und der Verlauf beider Phasen sich über einen größeren Zeitraum erstrecken, als die am Gastrocn. des Kaninchens (Fig. 17) gemessenen Kurven.

$\left.{ }^{1}\right)$ Verlauf und Theorie des Elektromyogrammes der Unterarmflexoren. Pflügers Arch. 1:9, 1909.

$\left.{ }^{2}\right)$ Beiträge zur Kenntnis des Frregungsvorganges der Nerven und Muskeln des Warmblüters. Zeitschr. f. Biologie 5:2, 1909. 
Wir haben es hier mit einer Kombination mehrerer zeitlich aufeinander folgender Reizwellen zu tun, welche über die zur Wirkung kommenden Muskelfasern von verschiedenen Muskelstellen aus ablaufen. Der größte Teil derselben läuft dicht hintereinander von der proximalen (dem Ellenbogen näheren) zur distalen $\mathrm{Ab}$ leitungsstelle und daher kombinieren sie sich $\mathrm{zu}$ einem zweiphasigen Gesamtstrom. Aus dieser Galvanometerkurve mußte

Fig. 18.

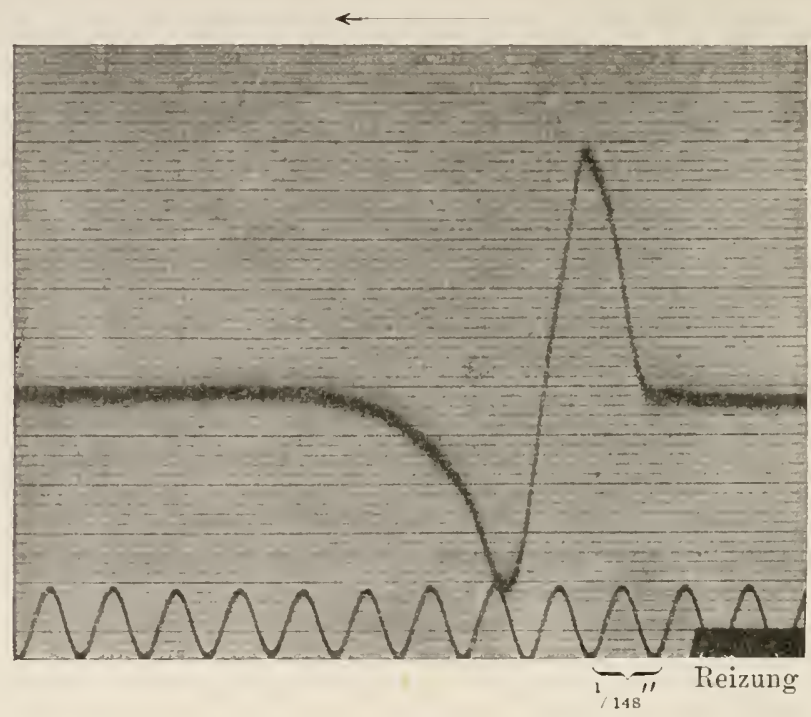

Doppelphasiger Aktionsstrom ron den Unterarmflexoren des Mlenschen.

Reizung des Nerv. medianus 8 cm oberhalb des Ellenbogens durch einen offnungssehlag ( $\mathrm{G}$ a rten).

freilich erst die wirkliche Stromeskurve konstruiert werden. Aber auch an der Galvanometerkurve erkennt man schon, daß der Anstieg der ersten Phase geringer als etwa $0,007^{\prime \prime}\left({ }^{1 / 148}{ }^{\prime \prime}\right)$ seiu muß, also kleiner als das gewöhnliche Latenzstadium der Kiontraktion $\left(0,01^{\prime \prime}\right)$. Es wäre daher wolıl vom Interesse, auch bei einem solchen Versuch gleichzeitig an den beiden Ableitungsstelleu die Kontraktionswelle photographisch (wie oben S.52) zu verzeichnen. L. Hermann suchte auch in seinem Versuche die 
Fortpflanzungsgeschwindigkeit der Reizwellen zu messen und bel'echnete den freilich nicht genau zu bestimmenden Wert derselben zu 10 bis $13 \mathrm{~m}$. Bernstein und Steiner hatten für die Geschwindigkeit der Kontraktionswellen am lebenden Kaninchen 6 bis $10 \mathrm{~m}$ gefundels. Beide Werte dürften wohl bei gleichzeitigen Messungen beider Vorgänge zusammenfallen.

- Um die vorliegende Frage rein zu lösen, wäre es also notwenclig, daß man vou einem möglichst langen, unverletzten parallelfaserigen Muskel eines lebenden Tieres, welches, um alle Nervenwirkungen auszuschließen, kuraresiert ist, zwei Stellen ableitet, die so weit voneinander entfernt sind, daß nachweislich die elektrische Reizwelle vollständig von der ersten Stelle abgelaufen ist, bevor sie an der zweiten beginnt, wenn man diesen Muskel an einem Ende durch einen einzelnen Induktionsschlag gereizt hat. Zugleich müßte man an beiden abgeleiteten Stellen die Kontraktionswellen aufzeichnen. Diese Aufgabe ist bisher noch nicht gelöst. Aber auch aus den bisherigen Ergebnissen läßt sich mit Deutlichkeit ersehen, daß Reizwelle und Kontraktionswelle gesonderte Vorgänge sind, und daß die Reizwelle mindestens mit ihrem ansteigenden Teil und wahrscheinlich anch mit dem Anfang des absteigenden Teiles der Kontraktionswelle in dem mechanischen Latenzstadium derselben voranschreitet.

Der gesamte Erregungsprozeß zerfällt daher in Miskel in zwei Teilprozesse. Die elektrische Zustandsänderung tritt in jedem Muskelelement zuerst ein, und erst wenn sie ihren Höhepunkt erreicht hat, fängt die mechanische Zustandsänderung der Kontraktion an, sich merklich zu entwickeln. Es ist ja möglich, daß das letztere auch schon in einer für unsere Hilfsmittel noch nicht wahrnehmbaren Weise anhebt, wenn die elektrische Zustandsänderung im Steigen begriffen ist. Wie dem aber auch sein mag, da die Maxima der elektrischen und mechanischen Zustandsänderung, der Reizwelle und Kontraktionswelle, in jedem Muskelelement zeitlich um mindestens $1 / 10$ Sek. auseinander liegen, so können beide Vorgänge in ihren Ursachen nicht ganz zusammenfallen, wenn sie auch miteinander in einem wesentlichen $\mathrm{Zu}$ sammenhange stehen. Da auch in den Nerven (s. unten) die elektrischen Prozesse bei der Erregung in ähnlicher Weise ablaufen wie im MInskel, ohne daßj damit eine mechanische Zustandsänderung wie die der Kontraktion verknüpft ist, so hat man nicht ohne Grund 
vermutet (Engelmann), daß die elektrischen Prozesse im Muskel an. eine andere Substanz geknüpft seien als die Kontraktionsprozesse. Von letzteren wissen wir, daß sie wesentlich der kontraktilen Substanz der Muskelfibrillen angehören, welche in dem Sarkoplasma, einer protoplasmatischen Substanz, eingebettet liegen. Ob aber eine solche Trennung sich durchführen lälit, muß noch unentschieden bleiben.

Über die Beziehung des elektrischen zum mechauischen Prozeß im Muskel sind mehrfache Untersuchungen angestellt worden. Beide Vorgänge verhalten sich der Reizstärke gegenüber ähnlich, indem sie mit ihrer Zunahme bis zu einem Maximum wachsen. Man hat aber auch geprüft, wie sich dieselben bei gleichbleibender Reizstärke mit zunehmender Belastung ändern. Von der Arbeitsleistung des Muskels ist es schon lange bekannt, daf dieselbe mit zunehmender Belastung cet. par. erheblich wächst, woraus man schließen muß, daß nit der zunehmenden Spannung bzw. Dehnung des Muskels die ausgelösten chemischen Energiemengen, welche sich in Arbeit und Wärme uusetzen, bis zu einer gewissen Grenze zunehmen. Wir haben es hier offenbar mit einer sehr zweckmäßigen Einrichtung eines physiko-chemischen Systems im lebenden (Organ zu tun, wodurch sich der Muskel den Anforderungen, die an ihn gestellt werden, in zweckentsprechender Weise aupaßt. Es war daher von Interesse, auch zu beobachten, wie sich der elektrische Prozeß im Muskel unter diesen Umständen verhält. Versuche hierüber müssen so angestellt werden, da@ bei einer Finzelzuckung des Muskels der ganze Ablauf der elektrischen und mechanischen Veränderung beobachtet wird. Da man aber, wie schon oben auseinandergesetzt, bei Ableitung des unverletzten Muskels au zwei Stellen wegen des geringen Abstandes derselben voneinander bisher niemals infolge der Interferenzen der Reizwellen ein reines Resultat erbalten konnte, so ist man genötigt, die negative Schwankung (einphasiger Aktionsstrom) des vom verletzten Muskel abgeleiteten Längsquerschnittstromes zu prüfen. Aber auch in diesem Falle würde man einen Fehler begehen, wollte man die Zuckung des ganzen Muskels mit der über den abgeleiteten Längsschnittpunkt (s. oben) ablaufenden Reizwelle vergleichen, vielmehr muß man, wie es im Versuch (s. Fig. 15, S. 52) geschehen ist, nur die über die abgeleitete Längsschnittstelle ablaufende Kontraktionswelle allein aufzeichnen. 
Unter diesen Bedingungen sind von Jensen l) mit dem Rheotom und Galvanometer und später von Tschermak2) mit dem Kapillarelektrometer Versuche angestellt worden.

Man hat nach Fick die Zuckung des Muskels, bei welcher er ein Gewicht hebt, die isoton ische Zuckung genannt, da seine Spannung während der Kontraktion nahezu dieselbe bleibt, dagegen diejenige Zuckung, bei welcher seine Enden befestigt sind, so daß er bei der Kontraktion dieselbe Länge behalten muß, die isometrische. Es ergibt sich nun bei der isotonischen Zuckung, daß mit zunehmender Belastung die Schnelligkeit des Anstieges und Gipfelhöhe der negativen Schwankung bis zu einer gewissen Grenze steigt und dann auch beim Abfall verhältnismäßig schneller sinkt. In der Fig. 19 sind nach Tschermak die Elektrometerkurven der negativen Schwankung bei verschiedener Belastung in ein Koordinatensystem eingetragen. Man ersieht daraus, daß ( $U$ U unbelastet, d. h. nur mit dem leichten sichreibhebel) mit zunehmender Belastung die Kurven an Höhe wachsen, und daß bei $200 \mathrm{~g}$ ein Maximum eintrat. Die römischen Zahlen geben die Reihenfolge der Beobachtungen an; durch die Abwechslung zwischen belastetem und unbelastetem Zustand kann man einigermaßen den Einflu der Zeit und der Ermüdung ausschließen ${ }^{3}$ ). Die berechneten Kurven der negativen Schwanknng bestätigen dieses Resultat Was die Lage der Maxima anbetrifft, so liegen sie entweder noch im Latenzstadium oder fallen in den

1) Über das Verhältnis der mechanischen und elektrischen Vorgänge im erregten Muskel. Pflügers Arch. 77, 107 (1899).

2) Über die Beziehung der negativen Schwankung des Muskelstromes zur Arbeitsleistung des Muskels. Pfügers Arch. S9, 289 (1902).

$\left.{ }^{3}\right)$ Die Form der Kurven zeigt mancherlei Abweichungen von der normalen. Es rührt dies daher, daß die Muskeln (musculi adductor. magnus et longus vom Oberschenkel des Frosches) nicht direkt, sondern vom Nerven aus gereizt wurden, weil direkte Reizung immer ein Einbrechen des Reizstromes in das Elektrometer zur Folge hatte. Es war also den oben aufgestellten exakteren Bedingungen des Versuches noch nicht ganz Genüge geschehen. Die Nerven breiten sich über $2 \mathbf{c m}$ weite Strecken aus, und daher haben wir allch hier eine Kombination von Reiz- und Kontraktionswellen. Die berechneten Kurven zeigen daher oft mehrere Maxina. Nichtsdestoweniger sind die Versuche zur Entscheidung der vorgelegten Frage über das Verbältnis der Reiz- und Kontraktionswellen gut geeignet, da beide Wellen in den untersuchten Muskelabschnitten örtlich genau zusammenfallen. 


\section{$-62-$}

Beginn der Kontraktionswelle; eine Verspätung derselben mit zunehmender Höbe ist nicht nachzuweisen. Der steiler abfallende Teil der Reizwelle liegt meist im aufsteigenden Teil der Kontraktionswelle, das langsamer abfallende Ende erreicht oft das Ende der letzteren. Nicht selten sinkt die Ordinatenhöhe im abfallenden

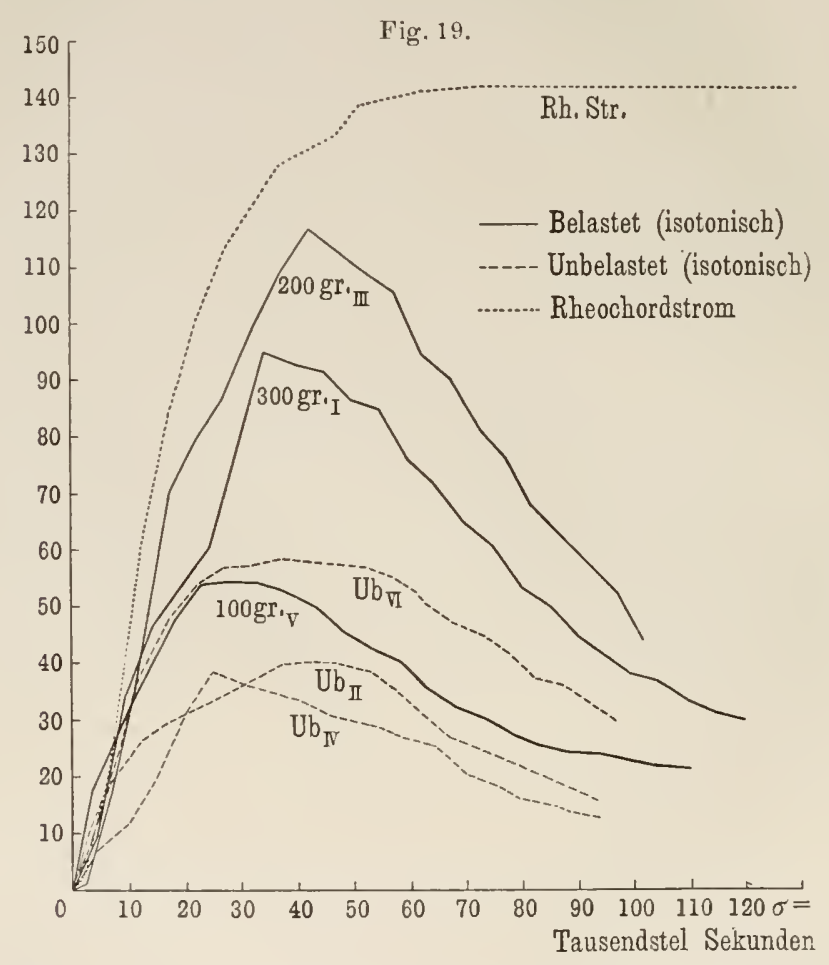

Negative Schwankung des Muskels bei isotonischer Zuckung und verschiedener Belastung. $U b$ unbelastet, $R h$, St $)$. Rheochordstrom zur Eichung (T schermak).

Ende der Reizwellon bei Belastung unter die des unbelasteten Muskels. Tschermak sagt: „Die negative Schwankung im Belastungszustande des Muskels zeigt demnach in aufsteigenden Teil und in der Gipfelregion Erhöhung, im absteigenden Teil daneben die Tendenz zur Erniedrigung, welche sich in relativ geringer Erhöhung, in Indifferenz, ja selbst in direkter Erniedri- 
gung zeigen kann." Dies ist der empirische Ausdruck der beobachteten Tatsache. Suchen wir uns diese durch eine Hypothese zu deuten, so köunte man sagen, daß währeud des aufsteigenden Teiles der Reizwelle eine Substanz entsteht, welche während des absteigenden Teiles infolge der mechanischen Leistung des Muskels verbraucht wird. Von dieser Substanz entsteht durch den Reiz um so mehr, je größer die Spannung des Muskels bei der Reizung ist. Um so mehr wird dann auch ron dieser Substanz während der Arbeitsleistung, beim Heben der Gewichte im aufsteigenden Teil der Kontraktion verbraucht. Die Gegenwart dieser Substanz erzeugt die Potentialdifferenz zwischen erregter und rubender Stelle des Muskels. Aus dieser Annahme würde es sich erklären,

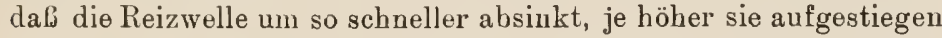
ist, so daf die Wellenlängen nicht mit der Höhe zunehmen, sondern nahezu dieselben bleiben. Aber es kann sich der chemische Prozeß im Muskel bei der Kontraktion nicht allein auf denjenigen beschränken, der mit der Reizwelle verknüpft ist, sondern es muß nachweislich die Menge der ausgelösten chemischen Energie noch von einem anderen Unstande abhängig sein. Dies folgt aus dem Verhalten der isometrischen Zuckung gegenüber der isotonischen, worauf Fick zuerst hingewiesen hat. Bei der isometrischen Zuckung, bei welcher der Muskel im einfachsten Falle in seiner natürlichen Länge, also ohne Spannung in der Ruhe an seinen Enden fixiert ist, wächst die Spannung erst während der Zuckung, und man findet durch Messung der Temperatur desselben mit Hilfe von feinen Thermosäulen, daß in solchem Falle weit mehr Wärme in demselben erzeugt wird, als bei der freien isotonischen Zuckung des unbelasteten bzw. schwach belasteten Muskels entsteht. Der chemische Umsatz muß also bei der isometrischen Zuckung in hohem Maße sich verstärken, und dies muß eine Folge der wachsenden Spannung sein, welcher der Muskel bei dieser Zuckung unterliegt. Nun wird aber, wie wir jetzt wissen, die chemische Energiemenge, welche der Reizwelle entspricht, schon im Stadium der Latenz, in welcher die Spannung noch nicht oder nicht merklich zunimmt, ausgelöst, und wenn dies der Fall ist, so wäre es daraus allein nicht zu erklären, woher der chemische Umsatz bei der isometrischen Zuckung so sehr viel größer ist als bei der isotonischen unter gleicher Anfangsspannung (Spannung des Muskels vor der Reizung). 
Man hat uun daher, um dieser Frage näher zu treten, auch den Ablauf der negativen Schwankung bei isometrischer Zuckung mit dem bei isotonischer Zuckung verglichen. Schon in älteren Rheotomversuchen hatte Lamansky gesehen, daß das Maximum der negativen Schwankungskurve bei der isometrischen Zuckung sich von der bei der isotonischen Zuckung nicht unterscheidet, was sich darauf zurückführen läßt, daß beidemal der Torgang bei

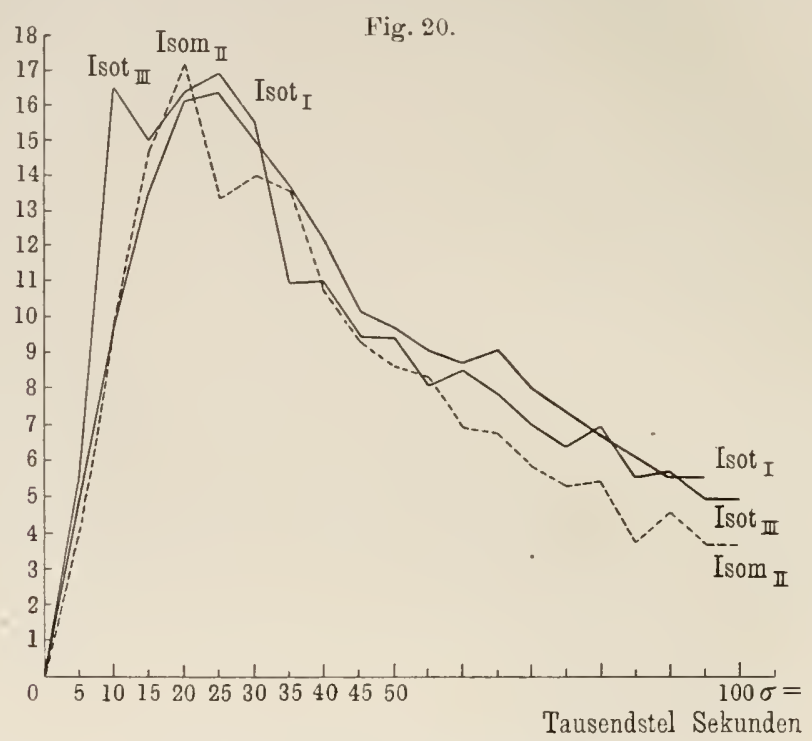

Verlanf der negativen Schwankung des Muskels bei isotonischer und isometrischer Zuckung (Tschermak).

derselben Spannung des Muskels ausgelöst wird und sein Maximum noch im Stadium der Latenz erreicht. Nun mußte aber der abfallende Teil der Kurve, welcher mit der Zuckung zusammenfällt, genauer untersucht werden, und da ergab sich aus den ziemlich übereinstimmenden Versuchen von Schenck, Jensen und aus denen, welche Tschermak bei partieller Isotonie und Isometrie mit Hilfe des Kapillarelektrometers anstelite, daß bei der isometrischen Zuckung die Kurve schneller abfällt als bei der isotonischen. In Fig. 20 sind die berechneten Kurven der negativen Schwankung für ein Beispiel der Versuche Tschermaks wiedergegeben. 
Es fragt sich nun, wie alle diese Resultate in ihrem $\mathrm{Zu}$ sammenhange zu deuten seien. Man könnte versuchen, durch die obige Annahme, daß bei dem elektrischen Prozeß eine Substanz entsteht, welche während der Kontraktion verbraucht wird, auch den Unterschied des chemischen Umsatzes bei Isotonie und Isometrie zu erklären. Das schnellere Absinken der Kurve in der Isometrie würde dann bedeuten, daß mehr von dieser Substanz verbraucht wurde. Indessen können wir uns mit einer solchen Deutung nicht begnügen, ohne auf die Tatsachen einzugehen, welche wir über den chemischen Prozeß bei der Muskeltätigkeit bereits kennen. Wir wissen, daß bei der Muskelarbeit Kohlehydrate (Zucker, Glykogen) verbrannt werden und daß dabei mehr Sauerstoff im Muskel verbraucht wird als in der Ruhe. Man ist geneigt, die Entstehung von Arbeit und Wärme im Muskel bei der Tätigkeit wesentlich aus der Verbrennung von Kohlehydraten zu erklären. Man weiß ferner, daß im Muskel bei der Kontraktion mehr $\mathrm{CO}_{2}$ entsteht als in der Ruhe und daß sich auch dabei eine gewisse Menge von Milchsäure bildet. Da Milchsäure aus Koblehydraten durch Spaltung z. B. bei der Milchsäuregärung in der Milch $\underset{\text { Zucker }}{\left(\mathrm{C}_{6} \mathrm{H}_{12} \mathrm{O}_{6}\right.}=\underset{\text { Milchsaure }}{\left.2 \mathrm{C}_{3} \mathrm{H}_{6} \mathrm{O}_{3}\right)}$ entstehen kann, so ist auch die Ansicht ausgesprochen worden, daß der chemische Prozeß bei der Kontraktion darin bestände, daß sich zuerst Milchsäure bilde und daß diese dann der Oxydation unterliege. Fick hat daher die Ansicht ausgesprochen, daß beim elektrischen Prozeß in der Latenz die Gegenwart der Milchsäure die Ursache der entstehenden Potentiale sei und daß diese daher bei der Kontraktion durch Oxydation der Milchsäure schnell abnähmen. Aus einer solchen Annahme würde sich wohl das elektrische Verhalten des IIuskels bei Isotonie mit wachsender Belastung erklären lassen. Die Menge der entstehenden Milchsäure wächst mit der Anfangsspannung, und mit der vermehrten Arbeit wächst auch die Schnelligkeit der Oxydation. Weniger gut läßt sich mit dieser Ansicht das Verhalten des Muskels bei Isometrio vereinigen. Es müßte hierbei z. B. beim unbelasteten Muskel dieselbe Milchsäuremenge entstehen wie bei Isotonie, d. h. die Maxima der entstehenden Potentiale müßten in beiden Fällen dieselben sein, und dann müßten die Potentiale bei Isometrie schneller auf Null absinken als bei Isotonie, was ja auch der Versuch bestätigt bat. 
Aber die Folge müßte sein, daß der isometrisch zuckende Muskel am Ende einer längeren Reihe von Zuckungen weniger Milchsäur'e enthielte als der isotonisch zuckende. Dies stimmt aber nicht mit der anderweitigen Beobachtung überein, daß nach Heidenhain die Säurebildung im Muskel mit der Arbeitsleistung zunimmt.

Eine etwas andere Vorstellung von diesem Vorgang ist von mir gegeben worden. Der Oxydationsprozeß in der lebenden Substanz kann nur dadurch zustande kommen, daß der molekulare Sauerstoff, welcher aufgenommen wird, in den aktiven atomaren Sauerstoff zerfällt, denn bei der Temperatur der Organismen, auch der warmblütigen, kann der molekulare Sauerstoff die organischen Verbindungen, wie Eiweiß, Kohlehydrate und Fett, nicht oxydieren. Auf welche Weise die Aktivierung des Sauerstoffs geschieht, ob durch Einwirkung von oxydierenden Fermenten, sogenannten Oxydasen, oder auf andere Art, weiß man nicht und mag bier unerörtert bleiben. Bei der Tätigkeit der Organe, insbesondere der Muskeln, muß demnach infolge der Reizung auch eine größere Menge aktivierten Sauerstoffs entstehen, als in der Ruhe sich beständig bildet. Auch wenn man voraussetzt, daß infolge der Reizung zuerst Milchsäur'e entstehe, so wäre doch zur Oxydation derselben aktiver Sauerstoff erforderlich. Also muß auch durch die Einwirkung des Reizes die Bildung des aktiven Sauerstoffs beschleunigt werden. Es würde daher schon dieser Vorgang, die Aktivierung des Sauerstoffs in der lebenden Muskelsubstanz, genügen, um die Potentialänderungen $\mathrm{zu}$ deuten, wenn man sich zunächst vorstellt, daß lebende Substanz, welche mehr aktiven Sảurstoff enthält, gegen diejenige, welche weniger enthält, elektronegative Spannung zeigt. Wie dies zu erklären ist, soll bei der Theorie der bioelektrischen Potentiale weiter behandelt werden. Die Höhe der Reizwelle an einer abgeleiteten Muskelstelle wäre hiernach ein Maß für die Konzentration des aktiven Sauerstoffs in der Muskelsubstanz. In der Latenz steigt diese Konzentration sehr schnell zum Maximum an und mit zunehmender Kontraktion, dem Wachsen der Arbeit oder Spannung, nimmt dieselbe dementsprechend durch Verbrauch des Sauerstoffs ab. So ist es zu verstehen, daß die Reizwelle im aufsteigenden Teil der Kontraktionswelle am stärksten sinkt, daß sie aber auch mit dem, letzten Ende noch in den absteigenden Teil hineinreichen kann, da auch in 
diesem noch Verbrennungsprozesse stattfinden. Ob bei dieser Oxydation zuerst eine Spaltung der Kohlehydrate in Milchsäure (oder andere Säuren, wie Fettsäure usw.) stattfindet, bevor die voll-

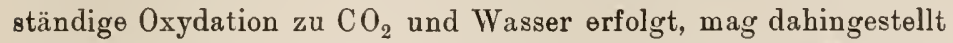
bleiben. So viel steht fest, daß die Muskelarbeit des Menschen und der höheren Tiere nicht durch Spaltung des organischen Brennmaterials der Muskeln allein geleistet werden kann, da mindestens 1/5 der gesamten im lebenden Körper umgesetzten Energiemenge der aufgenommenen Nahrung in mechanische Arbeit der Muskeln verwandelt werden kann und die Spaltungen der organischen Stoffe ohne 0xydation viel zu geringe Energiemengen liefern ${ }^{1}$ ). Ans der gegebenen Hypothese folgt nicht nur, daß bei Isotonie mit zunehmender Belastung die Reizwelle höber ansteigt und in der Kontraktion mit wachsender Arbeit schneller sinkt, sondern auch, dal bei Isometrie mit zunehmender Spannung die Reizwelle schneller absinkt als bei Isotonie. Was die Bildung der Säuren (Milchsäure usw.) anbetrifft, so findet dieselbe nach Ansicht von Hoppe-Seyler überhaupt nur bei Sauerstoffmangel im ausgeschnittenen Muskel, nicht aber bei genügender Sauerstoffzufuhr innerhalb des lebenden Körpers statt. Nur nach anhaltenden tetanischen Kontraktionen oder langdauernden Reihen von Zuckungen ist die Säurebildung überhaupt erst chemisch nachweisbar. In solchem Falle ist aber immer ein Mangel an Sauerstoff eingetreten, und dies mag auch im lebenden Körper, trotz beschleunigter Atmung, bei sehr starker und ermüdender Muskelarbeit mehr oder weniger der Fall sein, nachweislich auch bei Krämpfen, wie im Strychninkrampf.

Wenn man hiernach daran festhalten wollte, daß schon in der Latenz eine Spaltung in Säuren stattfände, welche bei der Kontraktion verbrennen, so käme man zu der widersprechenden Folgerung, daß bei isotonischer Zuckung mehr Säure übrig bliebe als bei der isometrischen, da die gebildete Säuremenge schon in der Latenz im Maximum der Reizwelle gegeben wäre. Denkt man sich dagegen, daß in der Latenz das Maximum der Reizwelle proportional der Konzentration des aktiven 0 sei, so würde daraus nur folgen, daß bei der isometrischen Zuckung mehr

1) Siehe Bernstein, Zur Thermodynamik der Muskelkontraktion, Pflügers Arch. 122, 159 (1908). 
davon verbraucht würde, als bei der isotonischen. Dies würde zu keinem Widerspruch führen; denn der nach der Zuckung übrig gebliebene aktive $\mathrm{O}$ würde sich in molekularen $\mathrm{O}_{2}$ zurückverwandeln köunen nach dem bekannten Schema: $20 \longleftrightarrow \mathrm{O}_{2}{ }^{1}$ ). Die übrig gebliebene fixe Säure dagegen könnte nicht etwa dazu dienen, wiederuın Moleküle von Kohlehydraten oder gar kompliziertere Moleküle zu erzeugen, sondern würde durch Anhäufung die Reizbarkeit herabsetzen. Wir wissen aber, daß eine Reihe isometrischer Zuckungen bei gleicher Anfangsspannung und gleichem Reize die Reizbarkeit weit mehr angreift, als eine Reihe isotonischer, während es nach der Säuretheorie umgekehrt sein müßte. Nach der 0 -Theorie würde also nach der Reizung in beiden Arten von Zuckungen zwar dieselbe $\mathrm{O}$-Menge disponibel sein, aber bei der isometrischen würde mehr davon verbraucht und somit auch mehr Brennmaterial verbrannt werden, als bei der isotonischen. Ob hierbei erst eine Spaltung in Säuren vorausgeht, oder ob die Bildung der Säuren nur bei O-Mangel erfolgt, oder ob sie auch bei genügendem 0 -Vorrat als Nebenreaktion auftritt, mag dahingestellt sein.

Nun sei noch erwähnt, daß man der Reizwelle áuch noch eine andere Bedeutung zugeschrieben hat. L. Hermann hat die Ansicht ausgesprochen, daß der Aktionsstrom der Reizwelle an jeder Stelle des Muskels oder Nerven, bzw. ihrer Fasern, selbst wiederum als Reiz für die benachbarten Stellen diene, so daß die Fortpflanzung der Erregung in der Muskel- und Nervenfaser auf einer solchen elektrischen Selbstreizung beruhen würde, die in ähnlicher Weise erfolge, wie die Reizung bei sekundärer Zuckung (s. oben S. 14). Wenn nun auch die Möglichkeit einer solchen Wirkung zugegeben werden kann, so erscheint sie von vornherein nicht gerade durchaus notwendig, da die Fortleitung eines chemischen Prozesses, aus welchem der Erregungs- und Kontraktionsvorgang doch zweifellos besteht, auch ohne eine solche erfolgen kann, wie wir dies bei chemischen Prozessen in einer zusammenhängenden Masse, besonders bei Explosionsprozessen, vielfach sehen. Doch wie dem auch sein möge, jedenfalls geht aus obigen Tatsachen hervor, daß der Reizwelle eine andere

1) Es könnte der Vorgang der O-Aktivierung noch auf andere Weise stattfinden, z. B. aus HO-Molekülen, welche sich ron einem komplizierten Molekuil (Eiweiß oder Oxydase) abspalten nach der Gleichung $2 . \mathrm{HO} \rightleftarrows \mathrm{H}_{2} \mathrm{O}+\mathrm{O}$. 
wichtige Bedeutung für den gesamten Erregungs - und Kontraktionsprozeß zukommt, und dab sie als Zeichen eines chemischen Prozesses anzusehen ist, der dem chemischen Prozeß der Kontraktion unbedingt vorauggehen muß.

Auch den zeitlichen Ablauf der Reizwellen bei Dauerkontraktionen des Muskels durch Reize gewisser Frequenz oder verschiedener Qualität und schließlich auch bei willkürlichen Erregungen kanu man mit Hilfe der beschriebenen Methoden genauer untersuchen. Es ist bekaunt, daß schuell aufeinanderfolgende Reize, z. B. Induktionsströme eines Induktoriums, eine kontinuierliche Dauerkontraktion, Tetanus, hervorrufen, wenn sie mit der hinreichenden Schnelligkeit, beim Frosch etwa 10 bis 15 mal, beim Säugetier etwa 15 bis $20 \mathrm{mal}$ in der Sekunde folgen. Diese Frequenz richtet sich wesentlich nach der Dauer der Einzelzuckung durch Einzelreize. Je länger die Zuckung dauert, um so geringer ist die Frequenz, welche schon einen kontinuierlichen Tetanus hervorbringt. Wenn das Intervall zwischen den aufeinanderfolgenden Reizen etwa gleich der Verkürzungsdauer ist, so wird der Muskel zwischen je zwei Reizen nicht merklich erschlaffen; die Kontraktionswellen summieren sich dann, und es wächst die Hubhöhe des Muskels im Tetanus zu einem höheren Maximum an, als die einfache Zuckung erreicht. Etwas anders verhalten sich dabei die Reizwellen. Dieselben verschmelzen nicht, selbst bei sehr frequenter Reizung, zu einem stationären Betrage, sonder'n bewahren eine diskontinuierliche Form. Dies hatte sich ja schon aus der Beobachtung des sekundären Tetanus ergeben und wurde auch mit Hilfe des Telephons ${ }^{1}$ ), welches man mit dem Muskel verband, selbst bis zu mehreren hunder't Schwankungen in der Sekunde festgestellt. Da aber die Dauer der Reizwelle im Muskel eine gewisse Länge hat, so müssen auch bei frequenter Reizung Summationen derselben eintreten. Dies läßt sich schon aus älteren Rheotomversuchen mit sehr schneller Rotation schließen und hat sich auch in späteren Versuchen mit dem Kapillarelektrometer deutlich gezeigt. Bei schnell aufeinanderfolgenden Reizen setzen sich die Elektrometerkurven der negativen Schwankungen (einphasiger Aktionsströme) so aufeinander

1) Bernstein und Schönlein, Sitzungsber. d. Naturf. Gesellschaft zu Halle, 8. Mai 1881. 
auf, daß sehr bald ein höheres Maximum erreicht wird, auf dessen Höhe sich aber noch Wellen, entsprechend der Reizzahl, aufsetzen (Burdon-Sanderson ${ }^{1}$ ). In Fig. 21 ist nach Versuchen von Tschermak ${ }^{2}$ ) eine Kapillarelektrometerkurve bei tetanischer Reizung des Nerven von dem Adduktor des Frosches abgebildet, Fig. 21.
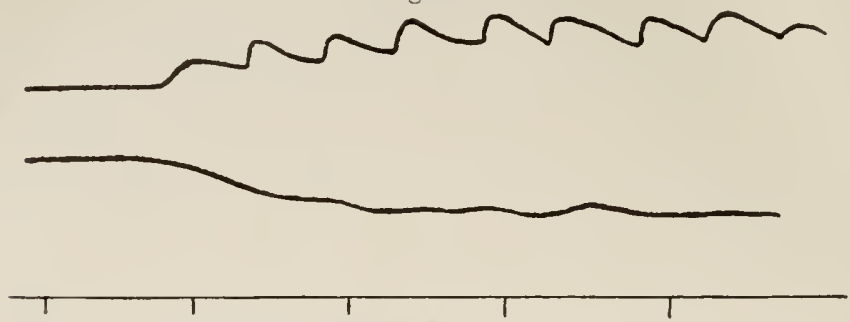

Isotonischer Tetanus des M. adductor des Frosches.

Obere Kurve negative Schwankungen am Kapillarelektrometer, mittlere Kurve

Kontraktion, untere Kurve Zeit in $0, \mathcal{L}^{\prime \prime}$ (nach Tschermak).

und zwar bei lokaler isotonischer Belastung und Ableitung der belasteten Stelle. Die obere Kurve gibt die Stromschwankuugen (nach oben gerichtet) an, die zweite die Kontraktion (nach unteu gerichtet), und die untere die Zeit in 0,1 Sek. Man erkennt, daß, bevor das Ende einer Reizwelle erreicht ist, schon die Fig. 22.

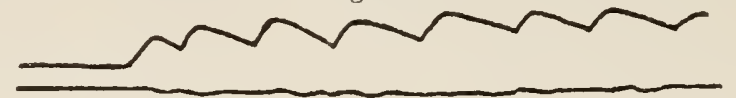

Isometrischer Tetanus desselben Muskels, Kurven ebenso wie in Fig. 21.

nächste einsetzt und daher zu einem höheren Betrage ansteigt. Im Vergleich hiermit sehen wir in Fig. 22 die Kurve bei isometrischem Tetanus von demselben Muskel. Auch hier tritt eine Summation ein, die aber ein deutlich geringeres Maximum gibt, als der isotonische Tetanus. Es bestätigt sich hierdurch eine schon vou du Bois-Reymond gemachte Beobachtung, $\mathrm{da} ß$ der isometrische Tetanus eine geringere Gesamtschwankung

1) The electrical response to stimulation of muscle and its relation to the mechanical response. Journal of Physiology, Vol. XVIII, p. 117 (1895).

2) 1. c., S. 61 , Anm. 2. 
in negativer Richtung gibt, als die freie Zusammenziehung (isotonische). Die Erklärung hierfür folgt aus dem Vorhergehenden. Beim isometrischen Tetanus ist der Stoffumsatz, also auch der Sauerstoffverbrauch ein viel größerer, als beim Tetanus des unbelasteten (oder wenig belasteten), sich frei zusammenziehenden Muskels. Dies geht unmittelbar aus der viel stärkeren Wärmeentwickelung im ersteren Falle hervor, was schon von Heiden hain und Fick sowohl für Zuckung wie für Tetanus bewiesen worden ist. Der stärkere O-Verbrauch beim isometrischen Tetanus bedingt daher ein schnelleres Absinken der Reizwellen als beim isotonischen und eine geringere Summation derselben. Dagegen muß sich im isometrischen Tetanus unstreitig mehr Säure bilden und anhäufen als im isotonischen, und wenn diese die Ursache der negativen Schwankung wäre, so müßte der Erfolg der umgekehrte sein.

Wie der Ablauf der Reizwellen sich gestaltet, wenn bei kūnstlicher Reizung des Nerven oder Muskels die Frequenz der Reize immer mehr zunimmt, ist durch Beobachtung mit den neueren graphischen Instrumenten noch nicht festgestellt. Voraussichtlich werden diese einer höheren Frequenz der Reizung nicht mehr hinreichend folgen können. Wie weit Nerven und Muskeln selbst auf höhere Reizfrequenz noch reagieren, soll an dieser Stelle noch nicht erörtert werden.

Auf anderem Wege kann man erfahren, wie sich der mechanische Zustand des kontrahierten Muskels bei verschiedener Reizfrequenz gestaltet. Er ist hierbei niemals ein ganz kontinuierlicher, obgleich man mit graphischen Hilfsmitteln keine Schwankungen der Länge und Dicke wahrnehmen kann. Dagegen nimmt man mit dem Gehör an dem kontrahierten Muskel einen Ton(bzw. Geräusch) wahr, den Muskelton, dessen Schwingungszahl mit der Reizzahl in der Sekunde genau übereinstimmt, wie zuerst Helmholtz gezeigt hat. Es gelingt mit Hilfo eines elektrischen Unterbrechers (akustischer Stromunterbrecher von Bernstein), Töne bis gegen 1000 Schwingungen in dem Muskel zu erzeugen ${ }^{1}$ ). Auch unter diesen Bedingungen ist also der mechanische Zustand im Muskel ein diskontinuierlicher und regelmäßig periodischer. Da jeder Reiz einen chemischen Prozeß auslöst, der unmittelbar von mechanischen Wirkungen gefolgt ist, so hat man diese Vorgänge nicht

1) Pflügers Arch. 11, 191 (1875). 
ohne Berechtigung mit Explosionen verglichen, die periodisch folgen und so einen Ton erzeugen. Wie sich nun dieseakustisch wahrnehmbaren Schwingungen der Muskelsub$\operatorname{stanz}$ zu den elektrischen Wellen verhalten, ist eine weitere Aufgabe der Untersuchung. Wenn man, wie oben schon erwähnt, die elektrischen Wellen mit einem $\stackrel{\bar{\Xi}}{.}$ Telephon wahrnimmt, und zugleich den Muskelton direkt 25 mit dem Ohre hört, so stimmen 궁 die beiden Töne vollständig überein ${ }^{1}$ ). Wie aber die zeitliche Lage der Wellen zueinander sich gestaltet, läbt sich auf diese Weise nicht entscheiden.

Die mechanischen und elektrischen Veränderungen des Muskels bei der willkürlichen oder reflektorischen Kontraktion sind ebenfalls der Untersuchung unterworfen worden. Helmholtz hatte an den Muskeln des Menschen bei willkürlicher Kontraktion das Muskelgeräusch genauer untersucht und durch Auflegung mitschwingender Federn eine Periode von 16 bis 20 Stößen in der Sekunde gefunden, die

$\left.{ }^{1}\right)$ Bernstein, Untersuchungen aus dem Physiologischen Institut zu Halle, Heft II, S. 183 (1890). 
in unserem Ohre einen Oberton von etwa 32 bis 40 Schwingungen erregen. Hieraus folgte, daß die zentralen motorischen Elemente des Gehirns und Rückenmarks keine kontinuierliche, sondern eine intermittierende periodische Erregung unserer Nerven erzeugen. Es war daher zu erwarten, daß man auch periodische Aktionsströme bei willkürlicher Kontraktion erkennen würde. Das ist auch in der Tat der Fall in Versuchen, welche in neuerer Zeit von Piper. und Garteu an den menschlichen Vorderarmmuskeln mit dem Saitengalvanometer angestellt sind. Doch stimmen die Beobachtungen nicht ganz überein. Piper will etwa 50 Erregungen in einer Sekunde gefunden haben. In Fig. 23 sind die von Garten ${ }^{1}$ ) an den Flexoren des Unterarmes erhaltenen Kurven abgebildet. Man sieht, daß die Kurve eine höchst unregelmäßige ist, aus großen und kleinen Wellen zusammengesetzt. Die Ursache hierfür liegt wahrscheinlich darin, daß bei der willkürlichen Innervation die Impulse keineswegs isochron den Nervenfasern zugeleitet werden, und daher die Reizwellen in den Muskelfasern nicht isochron ablaufen. Deshalb werden mannigfache Summationen und Differenzen auftreten, abgesehen von dem Einfluß der schon oben bei künstlicher (isochroner) Reizung der Nerven erwähnten Ausbreitungen der Nerveneintrittsstellen. Es ist daher erklärlich, daß man scheinbar eine größere Zahl von Wellen vorfindet, als dem natürlichen Muskelton entspricht, denn bei letzterem kann die Ungleichzeitigkeit der Erregungen keine Änderung der Tonhöle herbeiführen. Die Frage der Periode des willkürlichen Impulses kann also durch die elektrische Untersuchung nicht entschieden werden. Ähnlich muß es sich auch bei reflektorischen Kontraktionen verhalten, die man an Tieren, namentlich im Strychnintetanus, untersucht hat. Während der Muskelton im Strychnintetanus mit dem natürlichen Muskelton übereinstimmt, zeigen die Kurven des Kapillarelektrometers viele kleine unregelmäßige Schwankungen, welche auf höhere langsamere Wellenaufgesetzt sind (Buchanan). Dittler gibt an, daß bei der natürlichen Innervation des Zwerchfelles beim Atmen am Kaninchen rhythmische Aktionsstromschwankungen erscheinen, welche eine Periode von 60 bis 70 in der. Sekunde zeigen. Doch kann man auch hier denselben Einwand erheben. Selbstverständlich könnte

$\left.{ }^{1}\right)$ Handbuch der vergleichenden Physiologie (Winterstein) 3, 119. 
auch die Periode der zentralen Erregung mannigfachen Schwankungen unterliegen. Man hat schließlich auch bei direkter mechanischer Reizung des Muskels und bei Reizung der Nerven durch Schließung eines konstanten Stromes (Schließungstetanus) periodische Erregungen am Muskel beobachtet und diese als Folge eines eigenen Rhythmus des Muskels gedeutet (Garten u. andere).

\section{Viertes Kapitel. \\ Fortsetzung und elektrische Vorgänge in anderen Organen.}

Auch am Nerven hat man mit den graphischen Instrumenten die Reizwellen aufzeichnen können. Gotch und Burch ${ }^{1}$ )

Fig. 24 a.

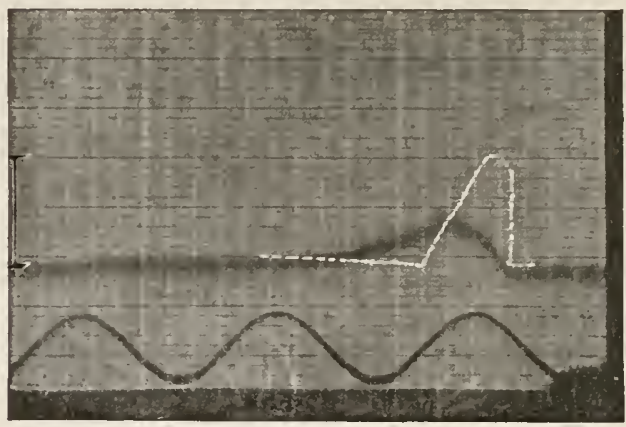

Einphasiger Aktionsstrom von 4 nebeneinander gelegten Nerven des Frosches (Längsquerschnittableitung), $18^{\circ} \mathrm{C}$ nach $\mathrm{Garten}$ (Handb. der vergl. Physiol. III, S. 137).

haben mit dem Kapillarelektrometer den einphasigen und zweiphasigen Aktionsstrom photographiert. Auch mit dem Saitengalvanometer sind Kurven dieser Ströme erhalten worden, die

1) Proe. of the Roy. Soc. 63, 310 (1898). 
aber bis jetzt noch nicht genauer analysiert werden konnten. In Fig. 24a ist nach Garten der einphasige Aktionsstrom des Froschnerven bei $18^{\circ} \mathrm{C}$ und in Fig. $24 \mathrm{c}$ der bei $32^{\circ} \mathrm{C}$ abgebildet. Die punktierte Kurve gibt uns eine ungefähre Berechnung des wirklichen Verlaufes, aus welcher folgt, daß die Zeit des Aufstiegs der Welle nicht größer als $0,55 \sigma$ ist. Nimmt man für den absteigenden Teil derselben (abgesehen von dem langsam ablaufenden Ende) etwa dieselbe Zeit an, so erhält man für die Dauer der Reizwelle etwas über $1 \sigma$. Dieser Wert ist nicht viel größer als der, weicher in den von mir zuerst mit dem Rheotom (siehe oben) angestellten Versuchen gefunden worden ist (etwa 0,8 $\sigma \mathrm{im}$ Maximum).

In Fig. $24 \mathrm{~b}$ ist der zweiphasige Aktionsstrom dargestellt mit der zugehörigen korrigierten Kurve. Hier war die Anstiegszeit der ersten Phase größer, etwa 1,2 б. Fig. 24 b.

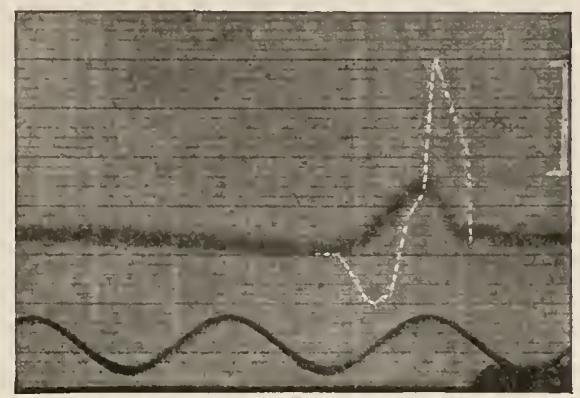

Doppelphasiger Aktionsstrom von 4 Nerven des Frosches. Ableitungsstrecke $22 \mathrm{~mm}$.

Daß, wio es den Anschein in diesem Falle hatte, die Reizwelle bei der Fortpflanzung niedrigør und länger wird, ist für den normalen Zustand nicht wahrscheinlich. Genauere Versuche über diesen Gegenstand sind noch zu erwarten. Dauer und Geschwindigkeit der Reizwelle hängt entschieden von der Temperatur ab, was schon aus den Helmholtzschen Versuchen über den Einfluß der Temperatur auf die Geschwindigkeit der Nervenerregung zu folgern ist. Kälte vermindert diese Geschwindigkeit beträchtlich; sie muß daher auch die Dauer der Reizwelle verlängern. Rheotomversuche von L. Hermann hatten dies bestätigt; neuere Messungen hierüber fehlen noch. Auch am Warmblüternerv sind einige Versuche mit dem Saitengalvanometer angestellt worden. In Fig. 25 ist der einphasige Aktionsstrom (negative Schwankung des Längsquerschnittstromes) des ausgeschnittenen Nerv. ischiadic. des Hundes bei $36^{\circ}$ nach einem Versuch von Garten abgebildet. 
Die Anstiegszeit der berechneten Welle beträgt etwa $0,7 \sigma$; die ganze Dauer (abgesehen vom Endteil) schätze ich zu etwa $3 \sigma$. Für einen gleichen Versuch am Nerv. ischiad. des Kaninchens gibt Garten für die Anstiegszeit $0,5 \sigma$ an, für die Gesamtdauer (mit dem sehr allmählich abfallenden Endteil) 7 б. An den Nerven des Murmeltieres hat Cremer bei einer Temperatur von $10^{\circ} \mathrm{C}$ eine viel längere Dauer der Welle gefunden (zu etwa $12 \sigma$, aber nicht analysiert), was sich durch die niedere Temperatur hinreichend erklärt. Es wäre wünschenswert, diese Versuche an

Fig. 24c.

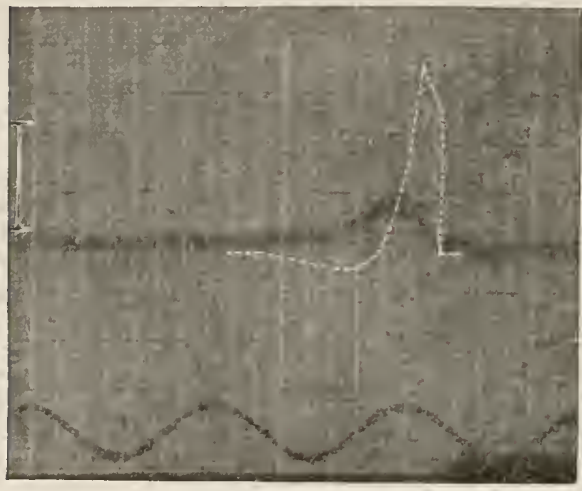

Einphasiger Aktionsstrom von 4 Froschnerven, $32^{\circ} \mathrm{C}$.

Nerven zu wiederholen, welche mit ihren zentralen Enden noch mit dem lebenden Körper in Verbindung stehen und daher sich in nahezu normalem Ernährungszustande befinden würden. Vermutlich würde die Reizwelle im lebenden Kïörper von kürzerer Dauer sein.

Was die absolute Größe der negativen Schwankung des Nerven anbetrifft, so hatten die von mir angestellten Rheotomversuche bereits im Gegensatze zum Muskel ergeben, daß die maximale Kraft viel größer werden kann, als die des Längsquerschnittstromes. Genauere Messungen dieser Art sind mit dem Saitengalvanometer noch nicht angestellt worden. Nach einem Versuch (Fig. 25) von Garten ist das Maximum der Welle über 3 Millidaniell, während für die Kraft des Längsquerschnittstromes beim Warmblüter nur 2 bis 3 Millidaniell angegeben wurden. Genauere Versuche dieser Art fehlen noch. Vor allem muß hierbei zu gleicher Zeit an denselben Nerven die Kraft des Längsquerschnittstromes mit der der negativen Schwankung verglichen werden.

Auf die Bedeutung dieses Verhältnisses für die Theorie kommen wir weiter unten zurück. 
Nicht in allen Nerven der Wirbeltiere und wirbellosen Tiere, wie in verschiedenen Muskeln derselben ist der zeitliche Ablauf und die Dauer der Reizwellen gleich. Vielmehr sehen wir, daß dieselben in gewissen Nerven auch höherer Tiere sehr viel langsamer verlaufen, wie in den Nervenstämmen der Extremitäten oder anderer Körperteile, in denen vornehmlich die motorischen Fasern der Skelettmuskeln und sensible Fasern der Haut vertreten sind. Über die Reizleitung in reinen Siunesuerven wußte man bisher nichts. Aus den elektrischen Untersuchungen am Riechnerven der Fische(Hecht) haben wir erfahren, daß in ihm die Reizleitung eine sehr langsame ist. Die Fasern dieses Ner'ven besitzen keineMarkhülle, im Gegensatz zu den meisten Gehirn - und Rückenmarksnerven. Sie sind auf einer niederen Entwickelungsstufe des Nervensystems stehen geblieben und dies scheint sich auch in einem viel langsameren Ablauf Fig. 25.

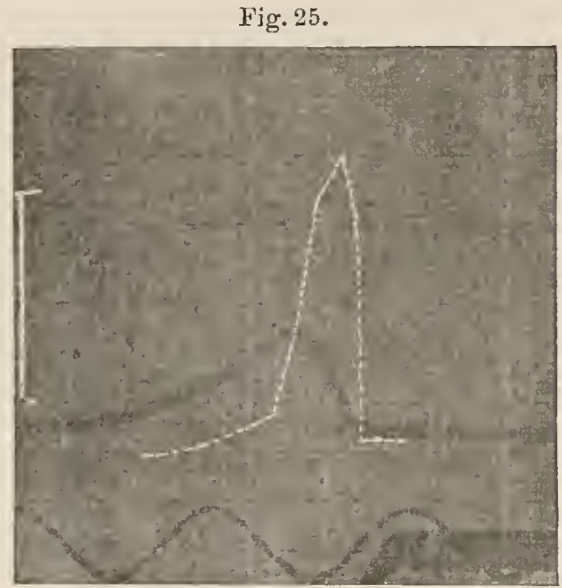

Einphasiger Aktionsstrom ron 2 Nerv. ischiadici des Huudes, $36^{\circ} \mathrm{C}$.

der Erregungsprozesse

zu dokumentieren. Bei der embryonalen Entwickelung der Nervenfasern, die aus den Nervenzellen hervorwachsen, sind dieselben zuerst marklos, später erst entwickelt sich die Markscheide, die den Achsenzylinder umhüllt. Man fand am Riechnerven des Hechtes eine Gesch windigkeit von 16 bis $24 \mathrm{~cm}$ bei $20^{\circ} \mathrm{C}$ und eine Dauer der Reizwelle von 0,4 Sekunden, mit einer Anstiegszeit von mehreren hundertstel Sekunden (Nicolai, Garten). Ganz ähnlich verhalten sich die marklosen Nerven niederer wirbelloser Tiere, so die Nerven von Muscheln (Anodonta, Flußmuschel) mit einer Geschwindigkeit von nur 10 bis $22 \mathrm{~mm}$ (Garten), während die Nerven von Cephalopoden (Octopus), Tiere, die sich lebhafter bewegen, eine geringere Dauer der Reizwelle von 8 bis $20 \sigma$ zeigen (Fuchs, Boruttau). 
Auch an höheren Tieren gibt es marklose Nerven mit träger, langdauernder und langsam fortschreitender Reizwelle. Dies zeigen z. B. die Versuche von Garten an den Milznerven des Pferdes. Der Anstieg dauert etwa $1 / 15^{\prime \prime}$, der Abstieg noch länger, die Leitungsgeschwindigkeit war etwa $46 \mathrm{~cm}$ bei 32 bis $36^{\circ} \mathrm{C}$. Aber im ganzen ist die Natur des Prozesses in allen Nerven dieselbe, prinzipielle Unterschiede sind nicht hervorgetreten. Von Einfluß auf die Dauer der Reizwelle ist dagegen die Art der Reizung. Bisher war immer als Reiz der fast momentan wirkende Induktionsschlag ${ }^{1}$ ) vorausgesetzt; die Dauer der Reizwelle ist von der Dauer dieser Schläge nicht abhängig. Eine länger dauernde Reizwelle beobachtet man dagegen bei der Schließung eines konstanten Stromes im Nerven. Dies konute schon mit Hilfe des Rheotomversuches festgestellt werden (Bernstein ${ }^{2}$ ). Leitet man an der Kathodenseite des zugeleiteten Stromes vom Nerven einen Längsquerschnittstrom ab, so findet man beim Schließen des Stromes eine starke, negative Schwankungswelle, kathodische Schließungswelle, vor. Dieselbe entspricht deutlich der starken Reizung, welche beim Schließen eines Stromes im Nerven (auch im Muskel) an der Kathode eintritt und sich an der starken Schließungszuckung eines an der Kathodenseite mit dem Nerven verbundenen Muskels zu erkennen gibt (s. unten Zuckungsgesetz). Diese negative Schwankungswelle kombiniert sich aber mit einem langsameren, austeigenden Polarisationsstrom (katelektrotonischer Strom s. unten), der sich mit abnehmender Stärke in der extrapolaren Strecke auf Seite der Kathode ausbreitet. An der Anode des erregenden polarisierenden Stromes entsteht beim Schließen keine Reizwelle, entsprechend dem Gesetz der polaren Erregung (s. unten). Dagegen tritt bei der Öffnung des konstanten Stromes an der Anode eine Reizwelle auf, nicht aber an der Kathode, in guter Übereinstimmung mit dem Gesetz der polaren Erregung. Leitet man an der Anodenseite des Nerven einen Längsquerschnittstrom ab, so bemerkt man beim Schließen des mittelstarken, erregenden, kon-

1) Auch diese sind bei gewöhnlichen Induktorien keineswegs als absolut momentan anzusehen. Die Schließungsinduktionsschläge dauern mehrere tausendstel Sekunden, die Öffnungsschläge sind erheblich kürzer.

2) Über das Entstehen und Verschwinden der elektrotonischen Ströme im Nerven und die damit verbundenen Erregungsschwankungen des Nervenstromes. du Bois' Archiv 1886, S. 197-250. 
stanten Stromes eine Reizwelle, welche an der Kathode entstanden und sich bis zur abgeleiteten Stelle über die Anode des konstanten Stromes fortgepflanzt hat, entsprechend der Schließungszuckung des mittelstarken aufsteigenden Stromes. Es entsteht aber auch zugleich der an der Anode sich ausbreitende anelektrotonische Strom im Nerven (s. unten 7. Kap.), welcher in positiver Richtung fließt. Ist der polarisierende Strom ein starker, so hemmt die Anode desselben die Fortleitung der Reizwelle, die negative Schwankung fällt aus, und es bleibt nur die nun stärkere positive Ablenkung durch den anelektrotonischen Strom übrig. Bei der Öffnung des Stromes

Fig. 26.

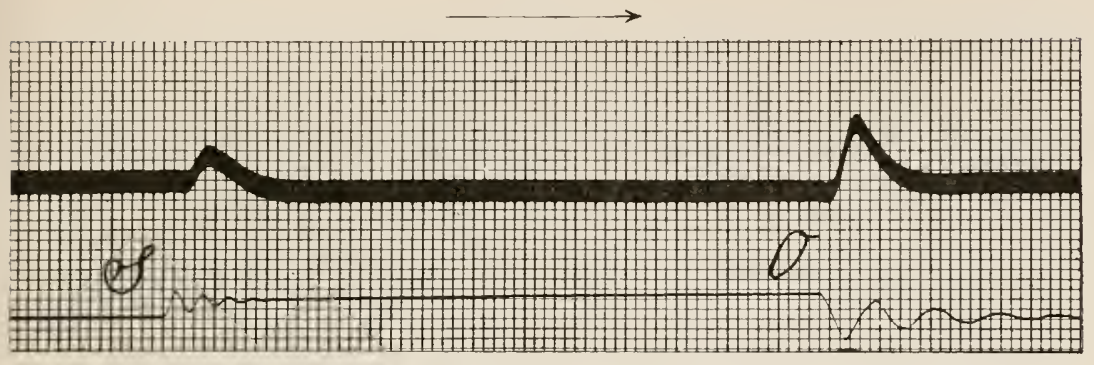

Schließungs- und Öffnungswelle am Kaninchennerven bei Reizung mit konstantem Strom. $S$ Scbließung, $O$ Öffnung des Stromes (Absz. 1 Skalenteil $=1 \sigma ;$ Ord. 1 Skalenteil $=374$ Mikrovolt). (Nach Einthqven, Verh. d. Ges. d. Naturf. 1911, S. 92.)

ist an der Anodenseite des Nerven eine Reizwelle nachweisbar, wenn die abgeleitete Strecke in größerer Entfermung von der polarisierten liegt (damit der anelektrotonische Strom nicht zu stark ist). An der Kathodenseite des Nerven ist die Reizwello, welche beim Öffnen des Stromes an der Anode entsteht, auch nachweisbar, solange der Strom mittelstark ist, während sie bei starkem Strom sich nicht über die Kathode fortpflanzen kann (entsprechend dem Zuckungsgesetz: Ruhe des Muskels bei Öffnung des starken, absteigenden Stromes im Nerven).

Systematische Versuche über diesen Gegenstand sind bisher mit dem Saitengalvanometer noch nicht angestellt. Einige Kurven sind bei Schließung und öffnung eines konstanten Stromes erhalten worden, so von Einthoven in obenstehender Fig. 26. Der Nerv vom Kaninchen war an der Anodenseite des konstanten Stromes abgeleitet, in $30 \mathrm{~mm}$ Entfernung von der Anode. Der 
Strom (obgleich 10 Volt) ist in diesem Falle als mittelstark bis stark anzusehen, denn die Schließungswelle ist kleiner als die Öffnungswelle. Die wahre Gestalt und Dauer der Reizwellen ist nicht berechnet. Die untere Kurve gibt an den Punkten $S$ und $O$ die Momente der Schließung und Öffnung des konstanten Stromes an. Für die Öffnungswelle, von der Anode ausgehend, wird sich eine Geschwindigkeit von etwa $30 \mathrm{~m}$ berechnen lassen. Die Schließungswelle, von der Kathode ausgehend (Reizstrecke nur $1 \mathrm{~mm}$ ), scheint an der Anode eine beträchtliche Verzögerung zu erleiden, entsprechend der schon von v. Bezold nachgewiesenen

Fig. 27.

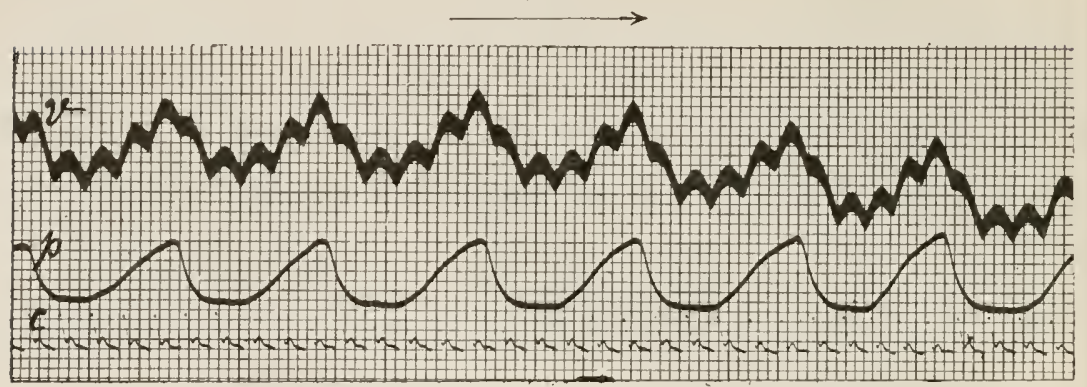

$v$ Reizwellen (Aktionsströme) vom peripheren Stumpfe des Nerv. vagus des Hundes. p Atemkurve, Erhebung Einatmung, Senkung Ausatmung. c Pulskurve der Arteria carotis (Absz. 1 Skalenteil $=0,2^{\prime \prime} ;$ Ord, 1 Skalenteil $=2,7$ Mlikrovolt). (Nach Einthoren, ebenda, S. 93.)

Terlangsamung der Reizleitung durch den Anelektrotonus. Ein schwacher anelektrotonischer Strom macht sich an der abgeleiteten Strecke durch schwache Senkung der Kurve zwischen den beiden Reizwellen bemerkbar.

Von besonderem Interesse sind auch Beobachtungen über natürliche Reizwellen des Nerven im lebenden Körper. Einthoven hat am peripheren Stumpf des Nerv. vagus bei Tieren Wellen beobachtet, wie sie in Fig. 27 wiedergegeben sind. Dieser Nerv versorgt mit einer Anzahl von Ästen Iunge und Herz. Die Lungenäste werden durch die Ausdehnungen der Lunge bei der Atmung rhythmisch gereizt. Bei jeder Aufblasung der Lunge sieht man daher an einem Galvanometer eine negative Schwankung des Längsquerschnittstromes an dem vom durchschnittenen Nerven abgeleiteten peripheren Stumpfe eintreten (Lew andow ski). 
Einthoreu hat diese Schwankungen mit dem Saitengalvanometer aufgezeichnet. Man sieht, daß bei jeder Einatmung des Tieres, welche durch Hebung der Kurve $p$ angegeben wird, eine Hebung der Galvanometerkurve eintritt, welche eine negative Schwankung bedeutet. Außerdem sieht man, daß die größeren Atmungswellen dieser Kurve noch eine Zahl kleinerer Wellen tragen, welche mit den Schwankungen der Pulskurve der Arteria carotis am Halse isochron sind. Eiuthoven erklärt sie aus der Reizung eines sensibeln Herznervens, des Nervus depressor, im Stamme des Nervus vagus, welcher nach Untersuchungen von Tschermak und Köster bei jedem Pulsschlag an seinen peripheren Enden durch Dehnung der Aorta am Herzen gereizt wird. Beim Kaninchen kann man beide Nervenäste trennen und erhält dann entweder nur die Atem- oder die Pulsschwankungen.

Weitere Beobachtungen über natürliche Reizwellen der Nerven sind bisher noch nicht ausgeführt. Doch ergeben sich hier maunigfache Aufgaben der Untersuchung. Ein gutes Objekt hierzu würde der wichtigste Atemnerv, der Zwerchfellnerv sein, dessen rhythmische Erregungen sich am zentralen Stumpf deutlich zeigen würden. Hierbei wird aber der Umstand zu beachten sein, daß eine streng isochrone Erregung aller Fasern des Nerven keineswegs zu erwarten ist, da die Aktion der Nervenzellen im Zentrum erst innerhalb eines gewissen Zeitraumes abläuft. Die Reizwellen werden daher nicht in allen Fasern koinzidieren, und ihre Zahl wird größer erscheinen, als sie in Wirklichkeit ist. Diese Bemerkung bezieht sich auch auf alle motorischen Nerven, wenn sie willkürlich oder reflektorisch in Aktion gesetzt werden, wie schon oben bei den willkürlichen Muskelkontraktionen erwähnt ist ${ }^{1}$ ).

Auch auf dieZentralorgane des Nervensystems läßt sich die elektrische Untersuchungsmethode ausdehnen. Namentlich sind von Gotch ${ }^{2}$ ) und Horsley mit dem Kapillarelektrometer

1) Dittler fand am Zwerchfellmuskel bei der Atmung periodische Aktionsströme von 60 bis 70 in der Sekunde. Dieselben blieben aber auch während der Apnoe (Aufhören der Atembewegungen bei künstlicher Atmung) bestehen.

$\left.{ }^{2}\right)$ On the mamalian nervous system, its functions and their localisation, determinated by an electrical method. Philosoph. Transaction 1891.

Bernstein, Elektrobiologie. 
Versuche hierüber an warmblütigen Tieren angestellt worden. Alle Nervenfaserstränge im Rückenmark und Gehirn geben einen Längsquerschnittstrom bei Verletzung, an welchem sich bei Reizungen negative Schwankungen zeigen. Es läbt sich nach dieser Methode der Verlauf der Nervenbahnen in den Zentren verfolgen. Neuere Untersuchungen in dieser Richtung mit dem Saitengalvanometer fehlen noch.

Ein wichtiges Objekt im Gebiete des Nerven- und MIuskelsystems bietet für diesse Untersuchungsmethode das $\mathrm{H}$ erz. Das Herz ist bekanntlich ein Hohlmuskel von kompliziertem Bau, der zur Erhaltung der Blutzirkulation dient. Er kann nicht willkürlich beeinflußt werden, sondern pulsiert rhythmisch infolge automatischer Erregung. Die Muskelfasern des Herzens unterscheiden sich in ihrem Bau und ihrer Funktion wesentlich von dener der Skelettmuskeln. Sie ziehen sich viel langsamer zusammen als diese und bestehen aus kleinen, länglichen, mit ibren Ausläufern untereinander verbundenen Zellen mit quergestreiftem, kontraktilem Inhalt.

Eine Pulsation des Herzmuskels ist als eine Kontraktionswelle anzusehen, die über den Muskel in bestimmter Richtung fortschreitet. Sie beginnt unter natürlichen Verhältnissen an der Einmündungsstelle der Venen in die Vorkammern, wo das Blut aus dem Körper in die rechte Torkammer und aus den Lungen in die linke Vorkammer einströmt. Von kier aus pflanzt sie sich auf die Vorkammern fort, und von dort geht sie nach einem bestimmten Rhythmus auf die Kammern über.

Nan kann an der abgeschnittenen, stillstehenden Kammer des Froschherzens leicht mit dem Galvanometer einen Strom vom Querschnitt und der Oberfläche (Längsschnitt) ableiten und bei jeder Reizung (z. B. durch Nadelstich) eine die Pulsation begleitende negative Schwankung wahrnehmen. Entsprechend der langsameren Kontraktion nimmt auch die negative Schwankung einen längeren Zeitraum in Anspruch als bei dem Skelettmuskel. Versuche mit Hilfe des Rheotoms (R. Marchand 1878) haben gezeigt, daß auch im Herzonuskel die Stromschwankung früher abläuft als die Pulsation, innerhalb einiger zehntel Sekunden. Das Verhältnis der elektrischen und mechanischen Vorgänge ist also ein ganz ähnliches wie in dem Skelettmuskel, die Pulsation erhebt sich erst während des abfallenden Teiles der negativen 
Schwankung zu ihrem Maximum und dauert beim Froschherzen oft 1 bis 2 Sekunden. An dem ausgeschnittenen unverletzten, spontan pulsierenden Froschherzen kann man mit gewöhnlichen Galvanometern, wegen ihrer Trägheit, keine regelmäßigen Ausschläge wahruehmen, wenn man von Vorkammern und Kammern ableitet. Dagegen konnte man mit Hilfe des Kapillarelektrometers den Ablauf der den Reizwellen entsplechenden Aktionsströme beobachten (Marey, Gotch und Burch, Waller). Im allgemeinen bestätigt sich der am Skelettmuskel festgestellte Satz, daß alle in Erregung befindlichen Teile der Muskulatur eines Organs sich elektronegativ gegen die ruhenden Teile derselben verhalten. Bei der normalen Pulsation in dem Rhythmus, Vorkammer-Kammer, entsteht daher zuerst ein Strom in der Richtung, Vorkammer $\rightarrow$ Kammer, und dann ein Strom, Kammer $\rightarrow$ Vorkammer. Doch ist die Kurve der Herzströme, wie namentlich neuere Versuche mit dem Saitengalvanometer gezeigt haben, durch den verwickelten Bau der Herzmuskulatur in mannigfacher Weise kompliziert. Waller konnte bereits mit dem Kapillarelektrometer auch die Aktionsströme des menschlichen Herzens durch Ableitung auf der Körperoberfläche nachweiseu. Mit dem empfindlichen Saitengalvanometer hat Ein thoven diese Ströme genauer photographisch aufgenommen, wovon Fig. 28 ein Beispiel zeigt. Die líurve $E P Q$ $R S T$ ist das sogenannte Elektrokardiogramm einer Pulsation, die obere Kurve $C R$ zeigt die gleichartig aufgenommene Pulskurve der Halsschlagader (Art. carotis), die nur wenig später als die Kammerpulsation erscheint. Es folgt hieraus, daß die Reizwellen des Herzens den Pulsationen um etwa 0,2 Sekunden vorausgehen. Die erste Erhebung $P$ ist auf die Pulsation der Vorkammer zu beziehen, $Q R S$ auf den Anfang und $T$ auf das Ende der Kammerpulsationen. Die Deutung dieser einzelnen Stromschwankungen ist eine sehr schwierige. Man hat dabei von dem Satze auszugehen, daß jede erregte Partie sich elektronegativ gegen unerregte Teile verhält. Aber bei der Reizleitung durch die Faserbündel der Vorkammern und Kammern kommt es zu einer mannigfachen Interferenz der Ströme, da die Reizwelle einer erregten Partie keineswegs ganz abgelaufen ist, wenn in den benachbarten und aufeinanderfolgenden Partien die Reizwellen beginnen. Aus dem verwickelten Bau der Herzmuskulatur beim Menschen und höheren Tieren hat man versucht, das Elektro- 
kardiogramm zu entziffern (Nicolai). Weitere genauere Untersuchungen an bloßgelegten Herzen von Tieren und Ableitung einzelner Faserbündel werden vielleicht bessere Aufklärung in dieser Richtung schaffen. Inzwischen hat man rein empirisch die verschiedenen Formen des Elektrokardiogramms unter physiologischen and pathologischen Bedingungen aufgenommen und so eine elektrodiagnostische Methode zur Untersuchung der Herzkrankheiten gewounen. Abweichungen vom normalen Elektrokardiogramm werden sich naturgemäß ergeben müssen, wenn die Reizleitung in den Faserbündeln des Herzens eine gestörte, irgend-

Fig. 28.

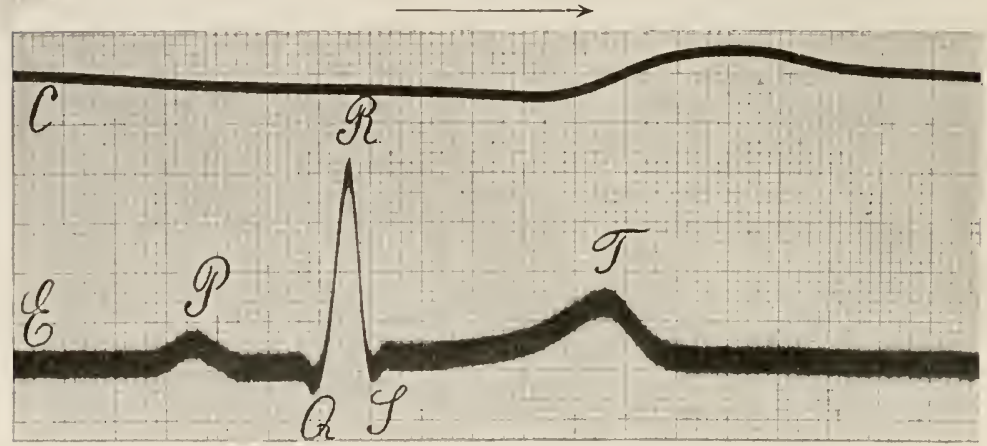

C Pulskurve der Arteria carotis, E Elektrokardiogramm des Menschen.

(Nach Einthoven, Verh. d. Ges. d. Naturf. 1911, S. 99.)

wie zeitlich oder örtlich veränderte ist, was durch organische Erkrankungen der Herzmuskulatur, wie der Klappen des Herzens bedingt sein kann. Ein Eingehen auf dieses spezielle Thema kann hier nicht Platz finden.

Noch langsamer als an den Fasern des Herzmuskels verlaufen alle Erregungsprozesse an den glatten Muskelfasern, welche sich in inneren Organen, in der Muskulatur des Darmkanals, der Blutgefäße, in den Harn- und Geschlechtsorganen usw. vorfinden. Ihre Zusammenziehung ist langsam und träge, und oft sieht man auch langdauernde, sogenannte tonische Zusammenziehungen auftreten. Sie bewirken auf diese Weise die peristaltischen Bewegungen des Magens und Darms, des Harnleiters usw., wie die tonischen Zusammenziehungen der Blutgefäße und den Tonus der 
Schließmuskeln der Blase und des Mastdarms usw. Die spindelförmigen Muskelzellen sind in Schichten neben- und hintereinander so angeordnet, daß sie in den röhrenförmigen Organen (Darm, Gefäße) ringförmig und zum Teil longitudinal gelagert sind. Wahrscheinlich sind sie durch Fortsätze miteinander verbunden, wodurch sich die Erregung überträgt, doch enthalten sie meist viel Nervenzellen und Nervengeflechte, wodurch sich ihre Reaktion mannigfach kompliziert.

Ein gutes Objekt der Untersuchung bietet der mittlere Teil des Froschmagens, der im wesentlichen aus ringförmigen Fasern zusammengesetzt ist. Der Längsquerschnittstrom desselben ist viel schwächer als der des Skelettmuskels, beträgt etwa zwischen 0,004 bis 0,01 D., und nimmt schnell an Stärke ab (Engelmann); das erklärt sich aus dem Absterben der kurzen Faserzellen am Querschnitt.

Eine genauere Untersuchung der negativen Schwankung und der Aktionsströme solcher glatten Muskelfasern ist bis jetzt noch nicht ausgeführt worden. Es ist anzunehmen, daß die elektrischen Prozesse in ihnen auch viel langsamer ablaufen als bei den Skelettmuskeln, aber auch den Zusammenziehungen vorausgehen werden. Dagegen hat man in neuerer Zeit mit dem Saitengalvanometer bei tonischen Zusammenziehungen solcher Muskeln (M. retractor penis des Hundes) rhythmische elektrische Wellen festgestellt (v. Brücke), von denen in $10^{\prime \prime}$ etwa zwei ablaufen. Am Harnleiter des Kaninchens sind bei mechanischer Reizung langsam ablaufende zweiphasische Ströme von einigen Sekunden Dauer gezeichnet worden. Der Verlauf ist etwa 1000 mal langsamer als am Skelettmuskel.

Bei niederen wirbellosen Tieren kommen glatte Muskelfasern der verschiedensten Formen vor, an denen man auch Ströme beobachtet hat. Die Schließmuskeln der Muschelschalen bieten hierzu ein gutes Objekt. An diesen ließ sich ein Längsquerschnittstrom nachweisen (Bernstein). An Muskeln eines Ringelwurmes (Sipunculus) konnte auch bei Nervenerregung negative Schwankung nachgewiesen werden (Fuchs). Da die Muskeln wirbelloser Tiere auch Übergänge zu quergestreiften zeigen, so werden sich hier vermutlich mannigfache Verschiedenheiten in dem elektrischen und mechanischen Verhalten darbieten.

Auch an der Netzhaut des Auges hat man Ströme und Schwankungsströme bei Belichtung desselben beobachtet (Holm- 
gren, Mc Kendrik). Die Netzhaut besteht aus drei Neuronen ${ }^{1}$ ). Das erste außen gelegene beginnt mit den lichtempfindlichen Stäbchen und Zapfen und reicht bis zur äußeren Körnerzellenschicht mit ihren Fasern, das mittlere besteht aus der imneren Körnerzellen-

$\gamma$ schicht und ihren Fortsätzen, und das dritte innen gelegene aus den Ganglienzellen der Netzhaut, ihren Fortsätzen und den Fasern des Sehnerven. Wenn ein Ruhestrom vorhanden ist, so geht er immer von der Stäbchen - und Zapfenschicht zur Sehnervenfaserschicht, und da beim Wirbeltierauge erstere außen und letztere innen liegt, von außen nach innen, am ganzen Auge von hinten nach rorn.

Fig. 29.

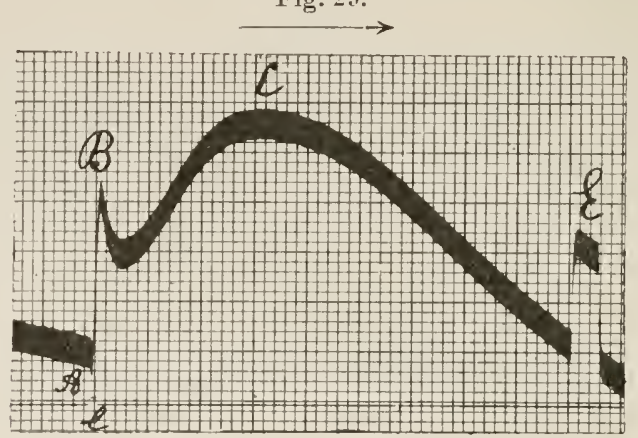

Photoelektrische Reaktion eines isolierten Froschauges.

Bei $l$ wird das Auge etwa $0,3^{\prime \prime}$ lang beleuchtet. A, B, $C$ Reaktion der 3 Substanzen (Absz. I Skalenteil $=0,5^{\prime \prime} ;$ Ord. 1 Skalenteil $=10$ Mikrovolt). (Nach Einthoven.)

Am höher entwickelten Áuge gewisser wirbelloser Tiere (Tintenfische), dessen Schichten umgekehrt liegen, geht auch der Strom umgekehrt. Ältere Beobachter (du Bois-Reymond, Holmgren, Kühn $\theta$ und Stein $\theta$ ) haben mit dem Galvanometer Schwankungen bei Belichtung auftreten sehen. In neuerer Zeit haben Garten und v. Brücke ${ }^{2}$ ) und Einthoven ${ }^{3}$ ) Versuche darüber mit dem

1) Das ganze Nervensystem ist aus "Neuronen " zusammengesetzt. Ein Neuron besteht aus einer Nervenzelle mit allen ihren Fortsätzen, von denen einer oder mehrere zu längeren Nervenfasern auswachsen. Diese Fasern enden mit einem „Endbäumchen“, durch welches sie sich mit Zellen verschiedener Organe (Muskeln, Drüsen usw.) oder anderen Nervenzellen in den Zentren des Nerveusystems verbinden.

$\left.{ }^{2}\right)$ Handb. d. vergl. Physiol., Bd. III, 2, S. 163 u. ff.

3) Verhandl. d. Gesellsch. deutsch. Naturf. 1911, S. 84 u. ff. 
Saitengalvanometer angestellt. Die Richtung von der Stäbchen- und Zapfenschicht nach der Nervenfaserschicht soll die positive beißen. Es erfolgt bei Belichtung zuerst eine kleine, sehr kurze negative, dann eine stärkere, längere positive Schwankung, die zwei Maxima zeigt, wie Fig. 29 nach Einthoven angibt, dann aber bald absinkt. Bei der Verdunkelung tritt ebenfalls eine positive Schwankung auf. Der Ruhestrom ist wohl daraus zu erklären, daß die Netzhaut von der Stäbchen- und Zapfenschicht aus abstirbt und daß demnach diese als Querschnitt gegen die Längsschnitte der Nervenfasern wirkt. Die Lichtreaktion will Einthoven aus einer Reaktion dreier Substanzen erklären, die ungleichzeitig agieren. Es ist vielleicht richtiger, dieselbe auf die drei Neurone der Netzhaut zu beziehen, die nacheinander in Aktion geraten, und deren Phasen miteinander mannigfach interferieren könnten. Es sind zur Erklärung dieser Vorgänge noch weitere Untersuchungen über' diesen Gegenstand erforderlich, und es läge zunächst die einfachere Aufgabe vor, die negative Schwankung des Sehnerven bei Belichtung des Auges zu verzeichnen ${ }^{1}$ ).

Fünftes Kapitel.

\section{Die Membrantheorie.}

Es ist klar, daß man die bioelektrischen Ketten nach denselben Prinzipien untersuchen mußte, nach denen man die physikalischen Ketten erforscht hatte. Bis dahin glaubte man wohl, daß es sich in den lebenden Organen um eine Entstehung elektrischer Energie besonderer Art handle, die in der toten Natur nicht vorkomme. Vor allem fehlen in den Organen alle Leiter erster Klasse, die Metalle, die gegen Flüssigkeiten hohe Kontakt-

$\left.{ }^{1}\right)$ Einen ähnlichen Versuch hat schon du Bois-Reymond, aber infolge der Unempfindlichkeit seines Multiplikators mit keinem deutlichen Erfolge, am Sehnerven und Auge der Schildkröte angestellt (Untersuch., Bd. II, 2, S. 522). 
potentiale erzeugen und zur Umsetzung chemischer Energie in elektrische dienen. Man konnte daher nur an sogenannte Flüssigkeitsketten denken. Aber es glückte lange Zeit nicht, solche herzustellen, welche ähnliche Wirkungen gaben. Nachdem die oben berichteten Untersuchungen von v. Helmholtz und Gibbs angestellt waren, nachdem die Ionentheorie von Arrhenius, Ostwald und Nernst ausgebildet war, war ein neuer Weg der Forschung auf diesem Gebiete geebnet. Den ersten Anstoß in dieser Richtung gaben Versuche von Ostwald ${ }^{1}$ ) an semipermeablen Membranen. Der Botaniker Pfeffer batte gezeigt, daß die von M. Tra ube zuerst hergestellte Niederschlagsmembran aus Ferrocyankupfer, welche bei Berührung von $\mathrm{CuSO}_{4}$ - und $\mathrm{F}_{\theta} \mathrm{Cy}_{6} \mathrm{~K}_{4}$-Lösung entsteht. nicht für alle Moleküle gleich durchlässig ist. Dieselbe läßt z. B. Moleküle des Rohrzuckers nicht durch, wie es auch bei der Plasmamembran der lebenden Pflanzenzellen der Fall ist. Sie zeigt sich undurchgängig gegen die Moleküle des $\mathrm{CuSO}_{4}$ und des $\mathrm{Fe}_{\mathrm{Cy}} \mathrm{K}_{4}$. Ostwald fand nun, daß diese Undurchlässigkeit für die Moleküle der Elektrolyte in vielen Fällen darauf beruht, daß das eine oder andere Ion derselben von der Membran nicht durchgelassen wird. Er stellte folgenden Versuch an: Ein n-Rohr wird mit $\mathrm{FeCy}_{6} \mathrm{~K}_{4}$-Lösung gefüllt, unten beiderseits mit Pergamentpapier verschlossen und in zwei mit $\mathrm{CuSO}_{4}$-Lösung gefüllte Gläser gestellt, in welche $\mathrm{Cu}$-Elektroden eintauchen. Auf der Scheidewand bildet sich nun eine Niederschlagsmembran aus Ferrocyankupfer. Die Kombination ist an sich stromlos, da sie ganz symmetrisch gebaut ist. Leitet man aber einen Strom hindurch, so bemerkt man, daß er stark sinkt und daß nach Öffnung desselben ein entgegengesetzter Polarisationsstrom auftritt. Der Sitz dieser Polarisationen kann nur an den Niederschlagsmembranen sich befinden. Die Erklärung hierfür ist nach Ostwald folgende:

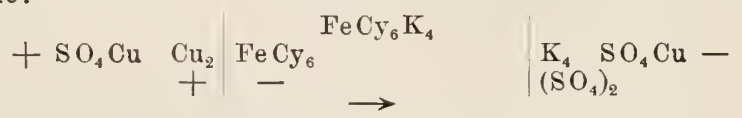

In dem beifolgenden Schema bedeuten die senkrechten Striche die Scheidewände, innerhalb deren die Lösung von $\mathrm{Fe}_{\theta} \mathrm{Cy}_{6} \mathrm{~K}_{4}$ sich

1) Elektrische Eigenschaften halbdurchlässiger Scheidewände. Zeitschr. f. physik. Chem. 6, 71 (1890). 
befindet. Geht der Strom in der Richtung des Pfeiles, so wanderu die $\mathrm{Cu}$-Ionen von links nach rechts, werden aber an der Scheidewand nicht durchgelassen; ebenso bleiben die $\mathrm{Fe}_{\theta} \mathrm{Cy}_{6}$-Ionen an der anderen Seite der Scheidewand stecken. Sie polarisieren daher diese Scheidewand gerade so, wie die an einer Metallplatte abgelagerten Ionen eines Elektrolyten in entgegengesetzter Richtung. Dagegen treten die $\mathrm{K}_{4}$-Ionen durch die rechte Scheidewand hindurch und verbinden sich dort mit den dahin geführten $\mathrm{SO}_{4}$-Ionen, die an sich auch nicht durchgelassen werden. Nach älteren Versuchen von Traube werden nämlich ClK-Moleküle durch diese Membran durchgelassen, nicht aber $\mathrm{K}_{2} \mathrm{SO}_{4}$-Moleküle. Dies kann nur daher rühren, daß die $\mathrm{SO}_{4}$-Ionen nicht durchtreten können.

Ostwald betrachtete daher die halbdurchlässigen Scheidewände solcher Art gewissermaßen als Ionensiebe. Er schloß daraus weiter, daß solche halbdurchlässigen Scheidewände der Sitz von Potentialdifferenzen werden müssen, wenn ein Elektrolyt durch Osmose hindurchgetrieben und eines der beiden Ionen darin zurückgehalten wird. Wenn z. B. auf der einen Seite einer Ferrocyankupfermembran sich $\mathrm{Fe}_{\mathbf{e}} \mathrm{C}_{6} \mathrm{~K}_{4}$-Lösung befindet, auf der anderen Seite Wasser, so werden die $K_{4}$ - Ionen hindurch zu diffundieren streben, während die $\mathrm{FeCy}_{6}$-Ionen zurückgehalten werden. Dadurch muß eine elektrostatische Kraft entstehen, die so weit anwächst, bis sie dem osmotischen Drucke das Glejchgewicht hält. Hierdurch müssen größere Potentialdifferenzen entstehen als in gewöhnlichen Flüssigkeitszellen, in denen die Bewegung der Ionen nicht gehemmt wird. Ostwald sprach daher in dieser Arbeit die Vermutung aus, "daß nicht nur die Ströme in Muskeln und Nerven, sondern auch namentlich die rätselhaften Wirkungen der elektrischen Fische durch die hier erörterten Eigenschaftender halbdurchIässigen Membranen ihre Erklärung finden werden".

Diese Anregung ist über ein Jahrzehnt lang unbeachtet geblieben, bis im Jahre 1901 und 1902 zwei Arbeiten erschienen, in denen der Ostwaldsche Gedanke einer osmotischen Ursache der tierisch-elektrischen Ströme aufgenommen wurde. OkerBlom ${ }^{1}$ ) setzte nach dem Beispiel von Nernst Flüssigkeitsketten

1) Tierische Säfte und Gewebe in physikalisch-chemischer Beziehung. II. Mitteilung. Die elektromotorischen Erscheinungen am ruhenden Froschmuskel. Pflügers Archiv S4, 191 (1901). 
zusammen, in denen ein Elektrolyt nach beiden Seiten mit ungleichen Ionengeschwindigkeiten diffundiert, z. B.:

$$
0,1 \mathrm{~K} \mathrm{Cl}\left|\mathrm{H}_{2} \mathrm{O}\right| 0,1 \mathrm{H} \mathrm{Cl} \mid 1,0 \text { Mannit | 0,1 K Cl. }
$$

Diese Kombination gibt einen Strom in der Richtung des Pfeiles, weil die Ionengeschwindigkeiten der. $\mathrm{HCl}$ im Wasser größer sind, als in der Mannitlösung. Oker-Blom nahm daher an, daß sich am Querschnitt des Muskels ein Elektrolyt bildet,

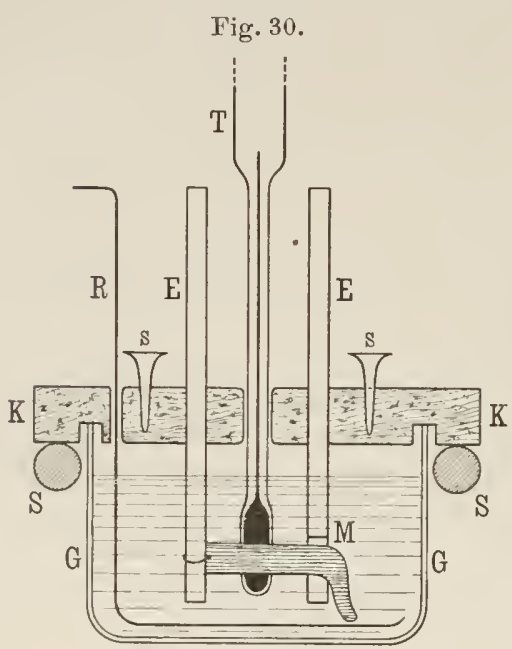

Versuch über den Einfluß der Temperatur auf die Kraft des Muskelstromes. wie es die Herunansche Alterationstheorie voraussetzt, und daß dieser mit verschiedenen Ionengeschwindigkeiten in die angrenzende Flüssigkeit und in die Muskelsubstanz diffundiert. Indessen verursachen Nichtleiter, wie Mannit und andere (Glycerin, Zuckerarten), nach Arrhenius bis zu 10 Proz., nur geringe Änderungen der Ionenbeweglichkeiten. $̈$ hnlich wirkte auch Gelatinezusatz.

Gleichzeitig unternahm ich $^{1)}$ die Untersuchung des Muskelstromes nach thermodynamischen Gesichtspunkten, wie sie oben Kapitel 2 auseinaudergesetzt sind. Es mußte zunächst entschieden werden, zu welcher Art von lietten die bioelektrischen gehören, und das konnte nur dadurch geschehen, daß man ihren Temperaturkoeffizienten bestimmte und das Verhältnis der elektromotorischen Kraft zur Temperatur genau feststellte. Schon von L. Hermann war beobachtet worden, daß diese Kraft mit der Temperatur steigt, aber es bedurfte nun einer genaueren Beobachtung dieses Verhältnisses. Wenn wir es im Muskel mit einer

1) J. Bernstein, Untersuchungen zur Thermodynamik der bioelektrischen Ströme. I. Pflügers Archiv 9:2, 521 (1902.) 
Konzentrationskette zu tun haben, so müßte sich nach der Theorie nicht nur ein positiver Temperaturkoeffizient ergeben, sondern es müßte sich dann herausstellen, daß die elektromotorischen Kräfte den absoluten Temperaturen annähernd proportional wachsen, vorausgesetzt, daß mit wechselnder Temperatur keine wesentliche Änderung in der Zusammensetzuug dieser Kette eintritt. Zur Untersuchung diente die in Fig. 30 abgebildete Einrichtung. In dem Glasgefäße $G G$ lag der Muskel unter Öl den mit 0,6 proz. $\mathrm{ClNa}$-Lösung getränkten Elektrodenstäben $E E$ aus gebranntem Ton mit Längs - und Querschnitt an; ein Thermometer $T$ gab die Temperatur an, welche durch ein Wasserbad variiert wurde. Die Versuche ergaben, daß $z$ wischen den Temperaturen 0 bis $32^{\circ} \mathrm{C}$ die elektromotorischen Kräfte den absoluten Temperaturen annähernd proportional sind, und zwar mit einer Genauigkeit, wie sie bei dem leicht veränderlichen Zustande eines lebenden Organes nicht besser erwartet werden kann. Zwischeu den Grenzen 0 und $20^{\circ} \mathrm{C}$ fallen die berechneten Werte etwas niedriger aus als die beobachteten; zwischen den Grenzen 18 bis $32^{\circ} \mathrm{C}$ ist das Umgekehrte der Fall, wie folgende Tabellen zeigen:

\begin{tabular}{|c|c|c|c|c|c|c|}
\hline \multirow[b]{2}{*}{ Nr. } & \multirow[b]{2}{*}{$T_{1}$} & \multirow[b]{2}{*}{$T_{2}$} & \multicolumn{3}{|c|}{ In Kompensatorgraden } & \multirow[b]{2}{*}{$\begin{array}{c}\text { Abweichung } \\
\text { Proz. }\end{array}$} \\
\hline & & & $E_{1}$ & $\begin{array}{c}E_{2} \\
\text { beobachtet }\end{array}$ & $\begin{array}{c}E_{2} \\
\text { berechnet }\end{array}$ & \\
\hline 1 & 276,7 & 290,5 & 459,65 & 498,1 & 482,57 & $+3,22$ \\
\hline 2 & 281,25 & 290,93 & 285,27 & 311,2 & 295,04 & $+5,14$ \\
\hline 3 & 280,37 & 292,61 & 449,60 & 483,98 & 469,24 & $+3,14$ \\
\hline $4 a$ & 278,8 & 292,8 & 441,57 & 482,40 & 463,74 & $+4,2$ \\
\hline $4 \mathrm{~b}$ & 276,00 & 292,92 & 406,75 & 435,85 & 431,71 & $+0,94$ \\
\hline 5 & 292,49 & 303,37 & 502,17 & 514,29 & 520,84 & $-1,27$ \\
\hline 6 & 292,04 & 304,01 & 450,45 & 467,66 & 468,96 & $-0,28$ \\
\hline
\end{tabular}

Hierin bedeuten $T_{1}$ und $T_{2}$ die absoluten Temperaturen, zwischen denen möglichst schnell gewechselt wurde; $E_{1}$ und $E_{2}$ die beobachteten Kräfte in Kompensatorgraden und $E_{2}$ ber. die nach der Proportion $T_{1}: T_{2}=E_{1}: E_{2}$ berechnete Kraft.

Noch bessere Übereinstimmung mit dieser Proportion wurde erzielt, wenn nicht zwischen zwei differenten Temperaturen gewechselt wurde, sondern eine allmähliche Erwärmung und Abküblung stattfand, und wenn zugleich die mit der Zeit auch bei 
konstanter Temperatur abnehmende Kraft in Rechnung gezogen wurde, wie z.B. folgender Versuch zeigt:

\begin{tabular}{|c|c|c|c|c|}
\hline $\mathrm{Nr}$. & $T$ & $\begin{array}{c}E \\
\text { beobachtet }\end{array}$ & $\begin{array}{c}E \\
\text { berechnet }\end{array}$ & \\
\hline 1 & 289,5 & 655,54 & - & \multirow{7}{*}{$\begin{array}{c}\text { Der Berechnung } \\
\text { wurden die Nummern } \\
1,4,7 \\
\text { zugrunde gelegt. }\end{array}$} \\
\hline 2 & 287,5 & 647,30 & 649,30 & \\
\hline 3 & 285,5 & 640,50 & 641,11 & \\
\hline 4 & 283,5 & 631,87 & - & \\
\hline 5 & 279,5 & 623,70 & 625,54 & \\
\hline 6 & 279,5 & 615,07 & 617,89 & \\
\hline 7 & 277,3 & 608,00 & - & \\
\hline
\end{tabular}

Bedenkt man, daß außer mit der Zeit auch durch die Einwirkung der abnorm hohen oder niederen Temperatur noch eine nicht kontrollierbare Änderung in der Konstitution des Muskels eintreten kann, so wird man die Übereinstimmung der beobachteten und berechneten Werte für ausreichend halten, um zu beweisen, daß der Muskelstrom eiu Konzentrationsstrom ist. Dazu kommt aber noch, daß auch die Ionenbeweglichkeiten nicht ganz unabhängig von der Temperatur sind, und daß sogar in einer physikalischen Konzentrationskette sich Abweichungen von der Formel durch verschiedene Temperaturkoeffizienten der Ionenbeweglichkeiten ergeben müssen.

Die hiernach von mir aufgestellte Membrantheorie ${ }^{1}$ ) der bioelektrischen Ströme beruht auf folgender Grundlage:

Nachdem von Pf ef fer gezeigt war, daß die Plasmamembranen der Pflanzenzellen als semipermeable anzusehen sind und sich diese Eigenschaft auch au den tierischen Zellen, wie Blutzellen, Muskelzellen usw., bestätigt hatte, wurde es sehr wahrscheinlich, daß sie sich nicht nur den Molekülen der gelösten Körper, sondern auch den Ionen der Elektrolyten gegenüber als semipermeabel verhalten und auf diese Weise Potentialdifferenzen erzeugen. Der Ostwaldschen Vermutung konnte daher in der Membrantheorie eine greifbare Gestalt gegeben werden, indem man die unter der äußeren Hülle der Muskelfasern (Sarkolemma) und den Nervenfasern (Neurolemma) liegende Plasmaschicht als eine solche Membran

I) 1. c., S. 542 . 
ansieht, wobei dahingestellt bleiben kann, ob auch jeder Fibrille der Faser eine solche Schicht zukommt. Dasselbe Bild paßt auch für die Konstitution aller Zellen im allgemeinen. Im Inneren der Plasmaschichten befinden sich Flüssigkeiten, in denen Elektrolyte und Nichtelektrolyte gelöst sind. Verletzt man an einer Stelle die Zellwand, legt man an Muskel- oder Nervenfaser einen Querschnitt an, so nimmt man an dieser Stelle die semipermeable Plasmamembran fort und die gelösten Substanzen können hier frei nach außen diffundieren. Fig. 31 gibt ein schematisches Bild

Fig. 31.

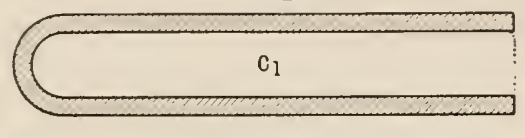

$\mathrm{C}_{2}$

$\mathrm{C}_{2}$

Schema einer Faser mit Plasmamembran, rechts verletzt.

dieses Verhaltens. Die schraffierte Hülle stellt die semipermeable Membran der Faser vor, an dem künstlichen Querschnittende ist sie fortgenommen. Befindet sich im Inneren der Faser ein Elektrolyt von der stärkeren Konzentration $c_{1}$ und außen in der umgebenden Flüssigkeit von der schwächeren Konzentration $c_{2}$, so

Fig. 32 a.

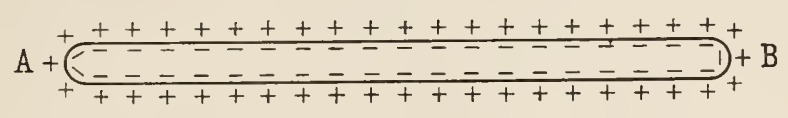

Unverletzte Faser mit elektrischer Doppelschicht, stromlos.

entstehen zwei Potentiale, ein Membranpotential an der Oberfläche der Membran und ein einfaches freies Diffusionspotential an dem Querschnitt, die einander entgegengesetzt gerichtet sind, von denen aber das erstere viel stärker ist als das letztere. Denkt man sich nämlich, daß, ähnlich wie bei der Ferrocyankupfermembran gegenüber $\operatorname{dem}\left(\mathrm{Fe}_{\mathrm{C}} \mathrm{J}_{6}\right) \mathrm{K}_{4}$, das positive Ion von der Plasmamembran durchgelassen und das negative Ion zurückgehalten wird, so entsteht an der Oberfläche der Faser eine Polarisation, wie sie Fig. 32 a darstellt, indem die positiven Ionen nach außen zu wandern streben, aber von den negativen Ionen im Inneren festgehalten werden. Solange die Faser unverletzt ist, besteht bei gleichartiger Beschaffenheit überall dasselbe 
Potential, d. h. der unverletzte Muskel ist unter dieser Bedingung stromlos, und auch seine Sehnenenden besitzen dasselbe Potential. Wird ein Querschnitt angelegt (Fig. 32b), so entsteht in der äußeren umgebenden Flüssigkeit jeder Faser, die in der Figur durch die äußeren Konturen angedeutet ist, ein Strom in der Richtung der Pfeile, von dem ein Zweigstrom nach außen abgeleitet werden kann.

Geht man von der einfachsten Annahme aus, daß es sich nur um einen Elektrolyten handelt, welcher in der Muskelfaser in größerer Konzentration enthalten ist als in der Außenflüssigkeit, so liegt es nahe, z. B. an die Kaliumsalze des Muskels zu denken.

Fig. $32 \mathrm{~b}$.

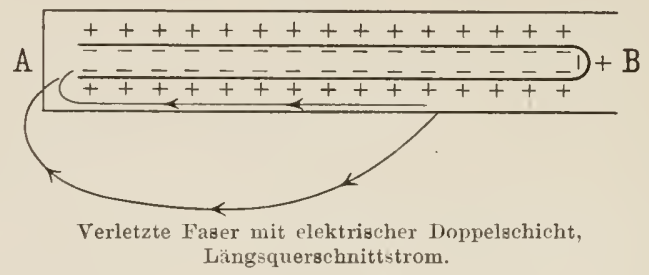

Man weiß seit langer Zeit, daß der MIuskel reich an Kaliumsalzen ist, während in der Blutflüssigkeit und in den aus ihr stammenden Gewebsflüssigkeiten nur sehr geringe Mengen von Kaliumsalzen vorkommen. Die semipermeable Membran der Muskelfasern ist also für die im Inneren befindlichen Kaliumsalze schwer durchlässig und verhindert auf diese Weise den Diffusionsaustausch dieser Salze gegen die Außenflüssigkeit und das Blut, was für die Funktion des Muskels zweifellos vou gewisser Bedeutung ist ${ }^{1}$ ). Folgt man nun der Ostwald schen Anschauung, daß die semipermeablen Membranen sich wie Ionensiebe verhalten, so kann dies daran liegen, daß zwar die Kaliumionen dieser Salze durch die Membran hindurchgehen können, daß dagegen ihre Anionen zurückgehalten werden. Nun ist der größte Teil des Kaliums in der Muskelsubstanz an Phosphorsäure gebunden, so daß die Möglichkeit vorliegt, daß das Kaliumphosphat $\mathrm{K}_{2} \mathrm{HPO}_{4}$ dasjenige Elektrolyt ist, dessen positive Ionen $\left(\mathrm{K}_{2} \mathrm{H}\right)^{+++}$und negative Ionen $\left(\mathrm{PO}_{4}\right)^{---}$das Membran-

1) Ganz ähnlich verhalten sich auch die roten Blutkörperchen der meisten Tiere, welche kaliumreicher sind als das Blutserum. 
potential erzengen. Wendet man zur Berechnung des Membranpotentials unter solchen Annalamen die Nernstschen Formeln an, so muß man von dem Ausdruck (4) (S. 35) ausgeben:

$$
\pi=K \cdot T \frac{u^{\prime}-v^{\prime}}{u^{\prime}+v^{\prime}} \log n a t \frac{p_{1}}{p_{2}} .
$$

Hierin sind $u \iota^{\prime}$ und $v^{\prime}$ die Beweglichkeiten der Ionen in der Membran. Für $\frac{p_{1}}{p_{2}}$ können wir auch das Verhältnis der molekularen Konzentrationen $\frac{c_{1}}{c_{2}}$ setzen. Nehmen wir nun an, daß die Beweglichkeit des Anions $v^{\prime}$ in der Membran gleich Null ist, so erhalten wir für das Membranpotential den Ausdruck:

$$
\pi_{m}=K \cdot T \cdot \log n a t \frac{c_{1}}{c_{2}} \text {. . . . . . . }
$$

Dieses Membranpotential können wir aber nicht direkt beobachten, sondern nur die Kraft des Längsquerschnittstromes messen. Es zieht sich also vom Membranpotential das Diffusionspotential am Querschnitt ab und setzt man dieses ebenfalls nach Formel ( 4$)$ :

$$
\pi_{d}=K \cdot T \frac{u-v}{u+v} \log n a t \frac{c_{1}}{c_{2}},
$$

so erhält man für die elektromotorische Kraft des Muskelstromes:

$$
E=\pi_{m}-\pi_{d}=K \cdot T\left(1-\frac{u-v}{u+v}\right) \log n a t \frac{c_{1}}{c_{2}}
$$

oder

$$
E=K \cdot T \frac{2 v}{u+v} \log n a t \frac{c_{1}}{c_{2}}
$$

Die Membrantheorie setzt die Präexistenz eines Potentials an der Oberfläche der Fasern voraus und erklärt dies durch das

1) Man kann nun auch von der Annahme ausgehen, daß das wirksame Elektrolyt sich in der Außenflüssigkeit befindet, und daß die Membran für das Kation (positive Ion) desselben nahezu undurchgängig sei, während sie das Anion (negative Ion) durchließe. Ein solcher Elektrolyt könnte ein Natriumsalz, z. B. das ClNa, sein, da man weiß, daß Natriumsalze im Muskel in viel geringerer Konzentration enthalten sind, als im Blute. Eine solche Annahme ist von Galeotti gemacht worden. Mlan würde natürlich zu denselben Formeln gelangen. Ferner kann man annehmen, daß es sich um einen Elektrolyten (1) im Inneren und einen zweiten (2) außen handle (Cremer), und dann hätte man auf 
Vorhandensein von Elektrolyten zu beiden Seiten der Membran. Sie ist daher, im Gegensatze zu der Hermannschen Alterationstheorie, als eine Präexistenztheorie anzusehen. Nach der Alterationstheorie müßte man nach unseren jetzigen Kenntnissen über die Flüssigkeitsketten die Entstehung eines Elektrolyten am künstlichen Querschnitt annehmen, wie es auch von Oker-Blom (s.oben) geschehen ist. Nach der Alterationstheorie würde der Potentialsprung sich am Querschnitte befinden, nach der Membrantheorie dagegen befindet sich der stärkere Potentialsprung $\left(\pi_{m}\right)$ am Längsschnitt $\left.{ }^{1}\right)$. Daß das letztere wirklich der Fall ist, läßt sich nun durch das Experiment erweisen. Wenn man einem unverletzten Muskel (Fig. 33 a) in der einen Hälfte die Temperatur $T_{1}$, in der anderen die Temperatur $T_{2}$ erteilt, so entsteht eine Potentialdifferenz zwischen diesen beiden Teilen des Muskels, bei welcher der wärmere Teil positiv gegen den kälteren wird. Ist $T_{2}>T_{1}$, so entsteht ein Thermostrom im Sinne der Pfeile. Dieser Strom läßt sich nur durch das Vorhandensein eines Membranpotentials erklären, das proportional der absoluten Temperatur steigt. Die Konzentrationen $c_{1}$ und $c_{2}$ in Formel (9) kann man als konstant ansehen, so daß man $\pi_{m_{1}}=$ const $T_{1}$ und

Grund der Berechnungen nach Planck bei gleicher Konzentration derselben die Formel:

$$
\pi=K \cdot T \log n a t \frac{u_{1}^{\prime}+v_{2}^{\prime}}{u_{2}^{\prime}+v_{1}^{\prime}}
$$

für das Membranpotential zu setzen. Sind $v_{1}^{\prime}$ und $u_{2}^{\prime}$ beliebig klein, so kaun die Kraft beliebig groß werden. Schließlich hat man die Membran nicht nach Ostwald als Sieb, sondern als zweites mit Wasser nicht (oder wenig) mischbares Lösungsmittel angesehen und die Theorie der Phasengrenzkräfte (s. oben S.37) darauf angewendet (Haber und Klemenziewicz). Indessen läßt sich durch eine solche Annahme der Membrantheorie eine brauchbare, mit den Tatsachen übereinstimmende Gestalt bisher nicht geben (s. Höber, Physikalische Chemie der Zelle und der Gewebe. 3. Aufl., 1911, S.482 u. 483). Tir werden daher im weiteren von der bisher gemachten Annahme zunäcbst ausgehen.

1) Man hat geglaubt, diese Streitfrage durch Schnittversuche am Muskel mit Hilfe eines Rheotoms (Hermann) oder des Kapillarelektrometers (Garten) entscheiden zu können. Versuche von Berustein und Tschermak durch Schnitt mit einem leitenden Knochenzahn haben aber gezeigt, daß diese Methode nicht beweisend ist, und daß der Strom schon mindestens $1 \sigma$ nach Anlegung des Querschnittes maximal vorhanden ist. 
$\pi_{m 2}=$ const ' $T_{2}$ setzen kann, und erhält für den Thermostrom des Muskels: $E_{t}=$ const $\left(T_{2}-T_{1}\right)$. In der Tat sind diese Ströme den Temperaturdifferenzelı nahezu proportional. Wenn man ferner

Fig. 33 a.

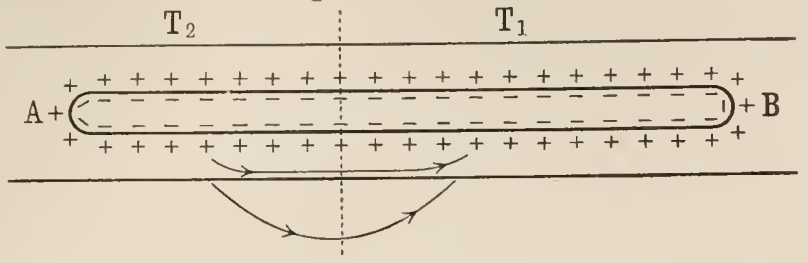

'Thermostrom der unverletzten Muskelfaser.

den Längsquerschnittstrom eines Muskels milit und der Längsschnitthälfte und Querschnitthälfte verschiedene Temperaturen gibt, wie Fig. $33 \mathrm{~b}$ angibt, so ist die Kraft des Stromes nur von der Temperatur der Längsschnitthälfte abhängig, nicht aber von der der Querschnitthälfte. Erwärmung der Längsschnitthälfte $B$ steigert, Abkühlung vermindert die Kraft proportional der absoluten Temperatur. Erwärmung und Abkühlung der Querschnitthälften hat dagegen nahezu gar keine Einwirkung auf die Kiaft.

Fig. $33 \mathrm{~b}$.

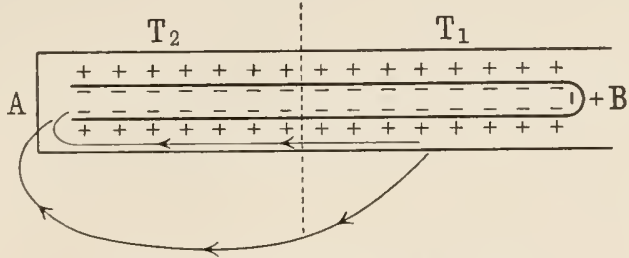

Thermostrom der verletzten Muskelfaser.

Daraus folgt unmittelbar, daß der wesentliche Potentialsprung der Stromkraft nicht am Querschnitt liegt, wie es die Alterationstheorie annahm, sondern am Längs-, schnitt, wie es die Membrantheorie fordert ${ }^{1}$ ).

1) Die Tatsache, daß wärmere Stellen des Muskels positiv gegen kältere sind, ist zuerst von L. Hermann gefunden worden, ebenso auch daß Temperaturänderungen des Querschnittes keinen Einfluß haben. Von Bernstein [Pflïgers Archiv 131, 589 (1910)] ist gezeigt worden, daß diese Thermoströme dem Gesetz der absoluten Temperatur folgen und sich aus der Membrantheorie erklären.

Bernstein, Elektrobiologie. 
Das Diffusionspotential $\pi_{d}$ am Querschnitt ist also verschwindend klein gegenüber dem Membranpotential $\pi_{m}$ am Längsschnitt, was sich daraus erklärt, daß die Beweglichkeiten $u$ und $v$ nicht sehr verschieden voneinander sind. Man kann daher für Berechnungen der elektromotorischen Kraft dieselbe praktisch dem Membranpotential

$$
\pi_{m}=K \cdot T \log \text { nat } \frac{c_{1}}{c_{2}}
$$

gleichsetzen, oder für $18^{\circ} \mathrm{C}$ und gewöhnliche Logarithmen:

$$
\pi_{m}=0,0575 \log \frac{c_{1}}{c_{2}}
$$

Es fragt sich, ob für irgend ein Elektrolyt des Muskels dieses Verhältnis der Konzentrationen $\frac{c_{1}}{c_{2}}$ innerhalb und außerhalb der Faser genügt, um die Kraft des Muskelstromes zu ergeben. Man hat dies von verschiedenen Seiten bezweifelt, weil die Konzentrationen, die in den Geweben vorkommen, nicht sehr groß sind. Indessen kommt es nicht auf die absoluten Werte, sondern auf das Verhältnis der Konzentrationen an. Wählt man das schon oben erwähnte $\mathrm{K}_{2} \mathrm{HPO}_{4}$ als Beispiel eines solchen Elektrolyten, nimmt nach Lehmann im Muskelfleisch 5,2 Prom. $\mathrm{K}_{2} \mathrm{O}$ an und rechnet alles $\mathrm{K}$ im Muskel nahezu auf dieses Salz, nimmt dagegen in der Außenflüssigkeit wie im Blutserum etwa 0,26 Prom. $K_{2} \mathrm{O}$ (Abderhalden), ebenfalls auf dasselbe Salz gerechnet, an, so erhält man für $\pi_{m}$ einen Wert von nicht weniger als 68 Millivolt, der der Kraft des Muskelstromes vollständig genügt (wenn man bedenkt, daß ein größerer Teil des $\mathrm{K}$ im Serum an $\mathrm{Cl}$ gebunden ist als im Muskel).

Nach Betrachtungen von $\left.\mathrm{H} \ddot{b} b \mathrm{r}^{1}\right)$ könnte man nun den wirksamen Elektrolyten finden, wenn man den Muskel in dio Lösung eines solchen brächte und dadurch die Konzentration desselben in der Außenflüssigkeit erhöhte. Dann müßte die Stromkraft abnehmen bzw. sich sogar umkehren. In der Tat werden Muskeln, welche mit einer 1,35 proz. Lösung von $\mathrm{K}_{2} \mathrm{HPO}_{4}$ behandelt sind, stromlos und zeigen sogar einen umgekehrten Strom von allerdings nur 1 bis 2 Millivolt (Overton). Das letztere erklärt sich

1) Pfï̈gers Archiv 106, 607 (1905). 
aber leicht daraus, daßj sie bei Zunahme der Konzentration der Kaliumlösungen bald gäuzlich absterben, wobei die Membrau für alle Ionen vollständig permeabel wird. Durch Auswaschen in physiologischer ClNa-Lösung erholt sich der Muskel und zeigt wieder starken regulären Strom. Der Versuch ist hiernach zwar kein direkter Beweis für die gemachte Voraussetzung, aber spricht doch in gewissem Grade dafür.

Anch wenn man in der Außenflüssigkeit als wirksamen Elektrolyten das $\mathrm{ClNa}$ annimmt, gelangt man zu Werten der Kraft von ähnlicher Größenordnung. Da die Muskelfaser nur geringe Mengen von Na-Salzen enthält, so kann dies sehr wohl darauf beruhen, daß ihre Plasmamembranen für Na-Ionen schwer durchgängig sind. Nehmen wir für den $\mathrm{NaCl}$-Gehalt der Außenflüssigkeit $c_{1}$ den des Blutserums zu 0,42 Proz. an und für die Muskelfaser $c_{2}$ den des Rindfleisches (nach Lehmann) zu 0,04 bis 0,09 Proz. (was sicherlich viel $\mathrm{zu}$ hoch ist), so erhält man bei Anwendung der Formel

$$
E=0,0575 \frac{2 u}{u+v} \log \frac{c_{1}}{c_{2}}
$$

den Wert von 31 bis 47 Millivolt ( $u=43,5$ und $v=65,5$ ). Schließlich könnte man beide Elektrolyte, $\mathrm{K}_{2} \mathrm{HPO}_{4}$ innen und $\mathrm{ClNa}$ außen, als wirksam ansehen, was durch Addition sehr viel höhere Werte ergeben würde ${ }^{1}$ ).

Diese Berechnungen sind selbstverständlich kein direkter Beweis, dab die genannten Elektrolyte die wirksamen sind, doch zeigen sie die Möglichkeit eines solchen Verbaltens. Indessen wird man noch an manche andere Möglichkeiten denken müssen, z. B. an Alkaliverbindungen der Eiweiße im Protoplasma der Zellen, welche man als Elektrolyte ansehen kann, von denen das leichter bewegliche Kation ausgesendet wird, während das schwer bewegliche Anion nicht durch die Plasmamembran hindurchtritt. Endlich

$\left.{ }^{1}\right)$ Bei Annahme zweier Elektrolyte, die durch die Membran getrennt sind, könnte man die Formel

$$
\pi=K T \cdot \log n a t u_{1}+v_{2}
$$

anwenden, welche Cremer für ausreichend hält (Handbuch d. Physiologie von Nagel, 1909, S. 875). Sie gilt aber nur, wenn die Konzentration beider Elektrolyte gleich und für jeden Elektrolyten auf der anderen Seite Null ist. 
möge auch nur hypothetisch daran zu denken sein, daß der von außen durch die Plasmamembran beständig eindringende Sauerstoff $\mathrm{O}_{2}$ hierbei in seine Ionen $\mathrm{O}^{-}$und $\mathrm{O}^{+}$zerfällt, von denen vielleicht $\mathrm{O}^{-}$eine größere Beweglichkeit besitzt als $\mathrm{O}^{+}$. Infolge der lelhaften Oxydation in den lebenden Zellen muß das Konzentrationsgefälle des Sauerstoffs von außen nach innen ein sebr bedeutendes sein. Obgleich nach den älteren Versuchen von du Bois-Reymond der Muskelstrom im luftleeren Raum bestehen bleibt, so ist es doch fraglich, inwieweit derselbe vom äußeren Sauerstoffdruck abhängig ist. Neuere Versuche hierüber feblen noch.

Von Höber ist die linwirkung verschiedener Salzlösungen auf die Muskelströme nach der Membrantheorie erklärt worden. Biedermann hatte gefunden, daB, wenn man eine Strecke eines unversehrten Froschmuskels in eine Kaliumsalzlösung taucht, diese gegen die übrigen Stellen negativ wird, d. h. es entsteht ein Strom wie von Längs- und Querschnitt. Wenn die Einwirkung nicht zu lange gedauert hat, kann der Strom durch Auswaschen des Muskels in einer physiologischen $\mathrm{Cl} \mathrm{Na-Lösung} \mathrm{wieder}$ verschwinden. Höber hat eine größere Reihe von Alkalisalzen daraufhin untersucht und hat beobachtet, daf einige in demselben Sinne wirken wie die Kaliumsalze, daß es aber auch andere gibt, welche in umgekehrtem Sinne wirken, so daß die eingetauchte Stelle positiv gegen die anderen Stellen wird. Es ist dies wesentlich von dem Kation abhängig, aber auch die Anionen haben einen Einfluß darauf. Man kann diese Salze nach einer bestimmten Reihe ihrer Katiouen und Anionen in folgender Tabelle

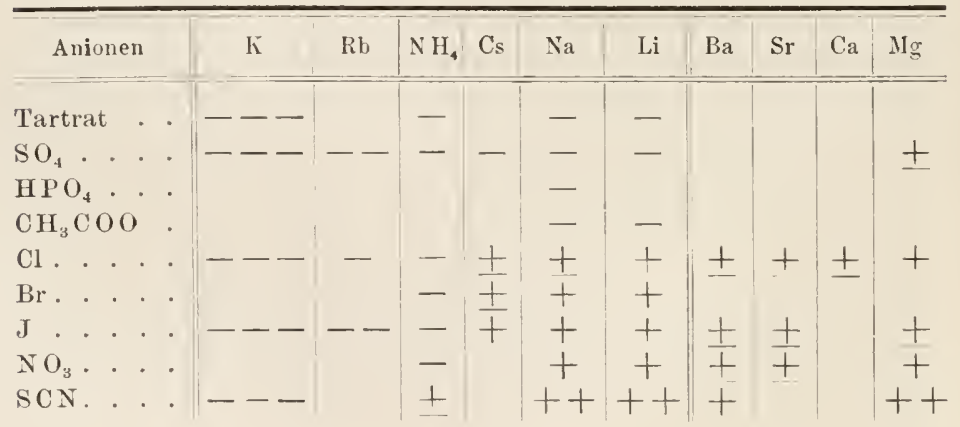


ordnen, in welcher ein Minuszeichen bedentet, daß die eingetauchte Stelle negativ, ein Pluszeichen, daß sie positiv wird, und ein Plus-Minuszeichen, daß sie neutral bleibt. Die Zahl der Zeichen entspricht der Stromkraft. Die Konzentration dieser Lösungen war immer äquimolekular uud dem Muskel isotonisch.

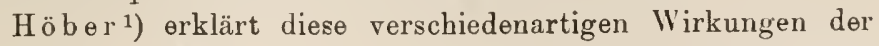
Salze auf den Muskel daraus, daß diejenigen, welche die behandelten Stellen negativ (-) machen, die Plasmamembran lockern, d. b. permeabler machen, daß aber diejenigen, welche jene Stellen positiv $(+)$ machen, die Plasmamembran verdichten, d. h. impermeabler machen, während alle diejenigen, welche keine Einwirkung ( + ) haben, die Plasmamembran nicht veränder'n. Overton hatte gefunden, daß Muskeln in einer 7 proz. Rohrzuckerlösung, welche sich zum Muskel isotonisch verhält, ihre Erregbarkeit bald verlieren, daß sie aber in gewissen Salzlösungen, wie $\mathrm{Cl} \mathrm{Na}$ und anderen, wiederkehrt, in anderen, wie Kaliumsalzen, dagegen nicht. Höber bemerkt nun, daß die Reihe dieser Salze, nach Kat- und Auionen geordnet, mit der obigen Tabelle gut übereinstimmt. "Alle Salze, welche einen regulären Rubestrom erzeugen, welche also die berührte Muskelpartie negativ machen, heben die Erregbarkeit rasch auf, während alle Salze, welche den stromlosen Zustand des unverletzten Muskels konservieren oder einen konträren Ruhestrom erzeugen, dem in Rohrzuckerlösung unerregbal' gewordenen Muskel die Erregbarkeit für längere Zeit wiedergeben können."

Die Tatsache, daß es Salzlösungen gibt, welche die Kraft des Muskelstromes erböhen und zugleich die Erregbarkeit steigern, ist eine gute Stütze für die Membrantheorie, denı sie kann wohl durch nichts anderes als durch eine Veränderung der Membran erklärt werden, welche das Membranpotential erböht. Betrachtet man die Tabelle (S. 100), so zeichnet sich unter den Kationen dieser Salze besonder's das $\mathrm{Mg}$, $\mathrm{Na}$ und $\mathrm{Li}$, unter den Anionen besonders das SCN aus. Es kommt sowohl auf die Kat-wie auf die Anionen der Salze hierbei an. Aber auch unter der Einwirkung dieser Salze befindet sich der Muskel nicht in einem normalen Zustand, denn bei längerer Einwirkung stirbt er darin schneller ab als in einer ClNa-Lösung.

1) Physikal. Chemie der Zellen und Gewebe, 3. Aufl., S. 412. 
Es muß bei dieser Gelegenheit die von 0 verton aufgestellte Lipoidtheorie der Zellmembranen erwähnt werden, nach welcher das Plasma der lebenden Zellen von einer Lipoidschicht eingebüllt sein soll, welche aus fettartigen Substanzen, wie Cholesterin, Lecithin usw., bestehen. Dies ist von Overton besonders daraus geschlossen worden, daß lipoidlösliche Körper, wie einwertige Alkohole, Aldehyde und Äther, rasch in die Zellen eindringen, während andere, welche Fette nicht lösen, wie der dreiwertige Alkohol, Glycerin, ferner Zuckerarten usw., obwohl sie in Wasser gut löslich sind, nur sehr langsam oder gar nicht eindringen. Die Lipoidtheorie und die elektrische Nembrantheorie haben sich beide fast zu gleicher Zeit auf Grund verschiedener Untersuchungen unabhängig voneinander entwickelt. Es kann aber nicht daran gedacht werden, die elektrische Membran mit einer Lipoidmembran zu identifizieren, vielmehr muß erstere wesentlich aus Bestandteilen des lebenden Plasmas zusammengesetzt sein. Da auch aus anderen Gründen Einwände gegen die ursprüngliche Overtonsche Lipoidtheorie erhoben worden sind und von vielen angenommen wird, daß die Plasmahüllen nicht nur aus Lipoiden, sondern auch aus Proteinen bestehen, so ist es wohl denkbar, dieselbe so zu modifizieren, daß sie sich mit der elektrischen Membrantheorie vereinigt, und daß sich aus ihr die osmotischen und elektrischen Erscheinungen an den Zellen gemeinsam erklären. Doch kann bier auf diesen Gegenstand 1) nicht näher eingegangen werden.

Ebenso wie auf den Muskel läßt sich die Membrautheorie auch auf die Nerven anwenden. Die Kraft des Nervenstromes steigt ebenfalls mit der Temperatur und in den Grenzen von 9 bis $18^{\circ} \mathrm{C}$, wenn man das Absterben mit der Zeit berücksichtigt, nahezu proportional der absoluten Temperatur, wie Tabelle B zeigt. Dagegen ist bei höherer Temperatur bis $32^{\circ} \mathrm{C}$ die $\mathrm{Ab}$ weichung von diesem Verhältnis eine größere als beim Muskel, und zwar bleibt die ITraft hinter der berechneten erheblich zurück, so daß meist bei 15 bis $18^{\circ} \mathrm{C}$ ein Maximum der Kraft auftritt. Es läßt sich aber sehr wabrscheinlich machen, daß diese Abweichung bei böherer Temperatur hauptsächlich auf eine Zunahme der Permeabilität der Membran mit steigender Temperatur

$\left.{ }^{1}\right)$ Siehe hierüber Höber, Physikal. Chemie der Zellen und Gewebe, 3. Aufl., S. 192 u. 488. 
zurückzufủhren ist. Wenn man unter dieser Annahme eine Berechnung ${ }^{1}$ ) ausführt, so kann man aus drei durch Messung erhaltenen Werten für die liraft die übrigen nach den gegebenen Formeln berechnen und erhält eine genügende Übereinstimmung mit dem Gesetz der Proportionalität der Kraft mit der absoluten Temperatur, wie Tabelle A zeigt.

T a belle A.

\begin{tabular}{|c|c|c|c|}
\hline \multirow[b]{2}{*}{ Nr. } & \multirow[b]{2}{*}{$T$} & \multicolumn{2}{|c|}{ liompensatorgrade } \\
\hline & & $\begin{array}{c}E \\
\text { Mlittel beob. }\end{array}$ & $\begin{array}{c}E \\
\text { ber. }\end{array}$ \\
\hline 1 & 289,35 & 782,63 & - \\
\hline 2 & 291,10 & 767,29 & 768,7 \\
\hline 3 & 293 & 771,37 & 772,15 \\
\hline 4 & 295 & 766,23 & 765,85 \\
\hline 5 & 297 & 757,95 & 759,23 \\
\hline 6 & 299 & 752,78 & - \\
\hline 7 & 301 & 744,83 & 744,76 \\
\hline 8 & 302,5 & 745,38 & 738,9 \\
\hline 9 & .305 & 731,20 & 728,56 \\
\hline 10 & 307 & 723,95 & 719,85 \\
\hline 11 & 309 & 712,00 & - \\
\hline
\end{tabular}

Tabelle B.

\begin{tabular}{r||l||c|c}
\hline \multicolumn{1}{l||}{} & \multicolumn{2}{|c}{ Kompensatorgrade } \\
\hline Nr. & $T$ & $\begin{array}{c}E \\
\text { korr. Mittel } \\
\text { beob. }\end{array}$ & $E$ \\
& & ber. \\
\hline \hline & & & \\
1 & 291,4 & 433,67 & - \\
2 & 298,25 & 430,7 & 429,97 \\
3 & 288 & 428,5 & 427,79 \\
4 & 286 & 423,67 & 424,08 \\
5 & 284 & 420,67 & 420,16 \\
6 & 283 & 418,03 & - \\
7 & 282 & 415,22 & 415,62 \\
8 & 281 & 408,59 & - \\
9 & 280 & 399,64 & 410,35 \\
10 & 279 & 395,00 & - \\
11 & 278 & 391,41 & 402,69 \\
& & &
\end{tabular}

Man könnte auch meinen, daß mit wechselnder Temperatur eine Änderung in der Konzentration der Elektrolyte in der Faser einträte; doch ist dies sehr unwahrscheinlich, da die Änderungen der Kraft mit wechselnder Temperatur immer reversibel sind und ein so schnelles Verschwinden entstandener Elektrolytmengen nicht möglich ist.

Auch an den Nerven kann man Thermoströme beobachten, wenn man von zwei möglichst gleichen Längsschnittstellen ableitet und eine Stelle erwärmt oder abkühlt. Von Verzár ${ }^{2}$ ) sind dieselben

1) Zugrunde gelegt wurde die Formel

$$
E=K \cdot T\left(\frac{u^{\prime}-v^{\prime}}{u^{\prime}+v^{\prime}}-\frac{u-v}{u+v}\right) \text { lognat } \frac{c_{1}}{c_{2}}
$$

unter der Annahme, daß $v^{\prime}=\beta . T^{2}$ ist, wo $\beta$ eine Konstante bedeutet. Je größer $v^{\prime}$ wird, um so mehr wächst die Permeabilität der ILembran.

$\left.{ }^{2}\right)$ Pflügers Archiv 14:3, 252 (1911). 
in letzter Zeit genauer untersucht worden. Zwischen 0 bis $20^{\circ} \mathrm{C}$ ist die wärmere Stelle immer positiv gegen die kältere. Bei hohen T'emperaturen treten unregelmäßige Resultate auf, die wahrscheinlich durch Schädigung des Nerven entstehen. Bei Ableitung eines Längsquerschnittstromes kann man durch Erwärmen oder Abkühlen des Längsschnittes allein den Strom verstärken oder schwächen. Aber im Gegensatz zum Muskel kann man auch beim partiellen Erwärmen oder Abkühlen des Querschnittes eine Änderung der Kraft hervorrufen, und zwar beim Erwärmen eine Schwächung, beim Abkühlen eine Verstärkung, doch sind diese Änderungen nicht so stark wie diejenigen, welche bei Temperaturänderungen des Längsschnittes erfolgen.

Diese Beobachtungen lassen sich auch im Sinne der Membrantheorie gut deuten. Die Änderungen der Kraft bei Temperaturänderungen des Längsschnittes erklären sich ebenso wie beim Muskel (s. oben S.96) durch Steigen des Membranpotentials proportional mit der absoluten Temperatur. Im Gegensatz zum Muskel muß man aber'annehmen, daß auch der künstliche Querschnitt des Nerven, entsprechend seiner besonderen Struktur, sich sehr schnell mit einer wirksamen Membran abgrenzt, die ebenso reagiert wie die Längsschnittmembran. Man möchte vermuten, daß die Ranvierschen Einschnürungen (s. oben S. 16) diese Quermembran bilden. I)ie Kraft des Längsquerschnittstronıes setzt sich dann aus zwei einander entgegenwirkenden Membranpotentialen zusammen. Temperaturerhöhung am Querschnitt muß daher die Kraft des Stromes schwächen, Erniedrigung sie verstärken. Man kann diese thermische Wirkung des Querschnittes daher nicht im Sinne einer Alterationstheorie auslegen, da es nach dieser sich umgekehrt verhalten müßte. Diese Auffassung stimmt mit der Erklärung der Eng elmannschen Versuche (s. oben S. 16) über dje Veränderungen der Kraft des Nervenstromes wohl überein. Ist der Strom mit der Zeit auf Null gesunken, so ist das Potential der Quermembran dem (inzwischen auch verminderten) Potential der Längsmembran gleich geworden. Die Erklärung der negativen Schwankung des Muskelund Nervenstromes ergibt sich nach der Membrantheorie daraus, daß die Membran als Teil der lebenden Substanz der Zellen an den chemischen Veränderungen teilnimmt, welche bei der Reizung vor sich gehen. Diese Veränderungen bestehen in Spaltungs- 
und Oxydationsprozessen der organischen Bestandteile, wobei $\mathrm{O}_{2}$ verbraucht und $\mathrm{CO}_{2}$ produziert wird. In derselben Richtung verlaufen auch die chemischen Prozesse beim Absterben der Organe, und es kann bei abnorm starker Reizung der Erregungsprozeß direkt in den des Absterbens übergehen, während bei normaler Reizung eine Restitution erfolgt. Auf die Übereinstimmung der chemischen Veränderungen des Muskels bei der Reizung und beim Absterben ist namentlich durch die älteren Arbeiten von L. Hermann hingewiesen worden. Nun wissen wir, daß beim Absterbeu der Fasern die abgestorbene Strecke sich negativ gegen die lebende verhält, im Sinne der Membrantheorie, weil die Membrau für gewisse Jonen der Elektrolyte permeabler wird. Daraus werden wir folgerı dürfen, daß auch bei der Reizung der Organe durch die chemische Veränderung die Permeabilität der Membran für diese Ionen zunimmt, und daß daher die erregte Strecke negativ gegen die unerregte wird. Mit anderen Worten, das Membranpotential nimmt bei der Reizung ab, um so mebr, je stärker die Reizung ist. Nach dem Schema ron Fig. 32 kamn man sich vorstellen, daß die negativen Ionen der Innenseite sich mit den positiven der Außenseite der Membran vereinigen. Diese Veränderung der lebenden Substanz pflanzt sich in der Fasej wellenförmig fort und hat an jeder Stelle eine gewisse Dauer, und so entsteht die elektrische Reizwelle, wie wir sie unter verschiedenen Bedingungen der Ableitung beobachten.

Eine Konsequenz dieser Theorie würde nun sein, daß die negative Schwankung eine maximale Grenze erreichen müßte, welche durch die Stärke des Membranpotentials gegeben wäre, und daß dieses bei der Reizung sich nicht umkehren könnte. In der Tat scheint dies nach den Rheotomversuchen Bernsteins am Muskel der Fall zu sein. In diesen Versuchen wurde das Rheotom (s. oben S. 43) auf das Maximum der negativen Schwankung eingestellt und beobachtet, daß auch bei stärkster Reizung die Ablenkung nicht unter die Abszissenlinie herabging. In späteren Versuchen von Burdon-Sanderson und Gotch ${ }^{1}$ ) mit Hilfe des Kapillarelektrometers schien es, daß am unverletzten Muskel die erste Phase des Aktionsstromes eine größere líraft bis zu etwa 0,1 Volt erreichen könne, als man sie am Längsquerschnittstrom

1) Journ. of Physiol. 12, 1892. 
$(\max .=0,080)$ beobachtet. Indessen hat man zu bedenken, daß der letztere sehr bald an Kraft abnimmt, namentlich wenn man den Muskel partiell durch Hitze abtötet (Wärmequerschnitt), wie es in diesen Versuchen geschah. Ferner ist ja auch nach der Membrantheorie die Kraft dieses Stromes die Differenz zwischen dem Membranpotential und dem Diffusionspotential am Querschnitt (s. oben S.95). Was aber wohl das Wesentliche ist, so muls nach Anlegung eines Querschnittes die ursprüngliche Kíraft des Stromes sehr schnell durch innere Polarisation sinken (s. unten Kapitel über innere Polarisation). Die wahre Kraft des Längsquerschnittstromes würde man daher nur finden, wenn man sie in den ersten Momenten nach Anlegen des Querschnittes messen könnte. Die Schnittversuche, welche von Tschermak und Bernstein angestellt worden sind (s. oben S.96. Anm. 1), sprechen in der Tat dafür, daß die Kurve der Kraft in den ersten tausendstel Sekunden in einer logarithmischen Form stark absinkt.

Daraus wird man schließen dürfen, daß man den wahren Wert des Membranpotentials nur vom unverletzten Muskel durch die maximale Kraft der ersten Plase des Aktionsstromes (vorausgesetzt, daß die zweite Phase noch nicht interferiert) erfährt. In maximo kann dieses Potential nur auf Null sinken, zur Annahme einer Umkebr desselben liegt keine Veranlassung vor ${ }^{1}$ ). Es bestätigt sich somit, daß die negative Schwankung des Längsquerschnittstromes in der Tat der elementare Vorgang ist und daß die Aktionsströme eben nichts anderes sind als die Kombination der negativen Schwankungen aller sukzessiv in Erregung geratenden Querschnittelemente der Faser.

Ganz dieselben Betrachtungen können wir auf die negative Schwankung des Nervenstromes und die Aktionsströme desselben übertragen. Am Nerven war bereits durch die Rheotomversuche von Bernstein festgestellt, daß die Kurve der

1) Die Versuche von Burdon-Sanderson und Gotch (Journ. of Physiol. 12, 1891) über diesen Gegenstand sind aus obigen Gründen meines Erachtens nicht entscheidend. Sie fanden sogar am M. sartorius für den Längsquerschnittstrom 29 und für den Aktionsstrom nur 25 M. D. Der am M. gastrocn. gefundene Aktionsstrom von 84 M.D. wird häufig vom Längsquerschnittstrom erreicht. Auch die späteren Resultate von Burdon-Sanderson (Journ. of Physiol. 23, 1898) über die Kraft der negativen Schwankung lassen sich nach obigem hinreichend erklären. 
negativen Schwankung weit unter die Abszissenlinie des Stromes herabsinken kann. Dies ist auch im wesentlichen daraus zu erklären, daß der wahre Wert der Kíraft des Nervenstromes sehr viel höher ist als wenige Minuten nach Anlegung des Querschnittes, weil dieselbe durch innere Polarisation sehr schnell absinkt. Dazu gesellt sich aber noch der von Engelmann entdeckte Vorgang (s. oben S. 16), daß die Nervenfaser zunächst vom Querschnitt aus nur bis an den nächsten Ranvierschen Schnürring abstirbt, und daß damit die manifeste Kraft des Stromes allmählich in eine latente übergeht. Dies erklärt sich nun nach den oben erwähnten Thermoversuchen am Nerven sehr gut aus der Annahme, daß die Ranvierschen Segmente der Nervenfasern von Quermembranen begrenzt sind, welche ebenso elektromotorisch wirken wie die Längsmembran. Der Längsquerschnittstrom muß also von Beginn an viel schwächer erscheinen als die monophasische Schwankung. Schließlich muß sogar, wenn das Potential der Quermembran zur Wirkung kommt, sich an die erste negative Phase der Schwankung eine zweite positive anschließen. Diese müßte am deutlichsten an einem noch gut erregbaren Nerven mit latenter Kraft auftreten, was bis jetzt experimentell noch nicht geprüft worden ist ${ }^{1}$ ).

1) Von E. Hering ist eine langsam eintretende positive Schwankung des Nervenstromes nach tetanischer Reizung beobachtet worden, und von ihm als Zeichen einer nach der Reizung einsetzender Assimilierung gedeutet worden. Nach Einzelreizungen konnte sie am Nerv. ischiad. nicht festgestellt werden. Dagegen hat Garten dieselbe an dem langsamer reagierenden Nerv. olfact. nach Einzelreizen gesehen. Auch an diesem Nerven findet man nach Anlegung des Querschnittes ein kontinuierliches Sinken des Stromes und Wiedersteigen desselben durch einen neuen Querschnitt vor. Es könnte auch dieses auf Entstehen einer Quermembran beruhen, obgleich diese Fasern keine Ranvierschen Einschnürungen zeigen. Es bedarf daher weiterer Versuche über diesen Gegenstand.

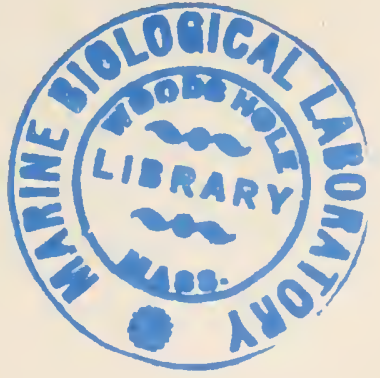


Sechstes Kapitel.

\section{Die elektrischen Organe.}

Die wunderbare Fähigkeit der Zitterfische, elektrische Schläge zu erteilen, ist schon seit alter Zeit bekannt. Dieselben bedienen sich dieses Mittels als Schutz und Waffe gegen die Angriffe anderer Tiere. Es gibt hauptsächlich drei Arten solcher Fische, erstens den Zitterrochen, Torpedo, welcher im Mittelmeer vorkommt, zweitens den Zitterwels, Malapterurus, welcher sich in den Flüssen Nordafrikas vorfindet, und drittens den Zitteraal, Gymnotus electricus, welcher in den Flüssen der tropischen Zonen Afrikas und Südamerikas lebt. Daß der Schlag des letzteren nach den älteren Berichten von $\mathrm{Humboldt}$ Pferde zu töten vermag, hat sich zwar nicht bestätigt, doch sind die Schläge desselben außerordentlich kräftig.

Diese Tiere besitzen elektrische Organe, welche durch Nerven versorgt und ebenso wie die Muskeln willkürlich in Tätigkeit versetzt werden. Beim Zitterrochen liegen diese Organe zu beiden Seiten des Kopfes (s. Fig. 34a) von den beiden Seitenflossen begrenzt, etwa handtellergrob und platt, die ganze Dicke des Körpers einnehmend, und werden von vier starken Nerven versehen, welche aus dem elektrischen Lappen des Gehirns stammen. Das Orgau ist aus sechsseitigen Säulen zusammengesetzt, welche, wie man auf dem Querschnitt Fig. 34b sieht, senkrecht zur Längsrichtung des Körpers dicht nebeneinander stehen. Diese zerfallen wieder der Quere nach in eine große Zahl von Scheiben, welche durch Querwände voneinander geschieden sind. Die Nervenäste dringen zwischen den Säulen überall ein und die Fasern derselben treten in die Querwände ein, um sich mit einer Seite der Scheiben zu verbinden. Diese Seite ist beim Zitterrochen durchgehends die Bauchseite. Sie wird, wie die Untersuchung gelehrt hat, beim Schlage negativ (s. Fig. 34 b).

Beim Zitterwels umgibt das elektrische Organ mantelartig den mittleren Teil des Körpers (s. Fig. 35). Es besteht ebenfalls ans 
Säulen, welche aber longitudinal gelagert sind und ebenfalls in viele Querscheiben zerfallen, durch deren Scheidewände die Nervenfasern eintreten. Beim Schlage wird das Kopfende des Organs negativ.

Fig. 34 a.

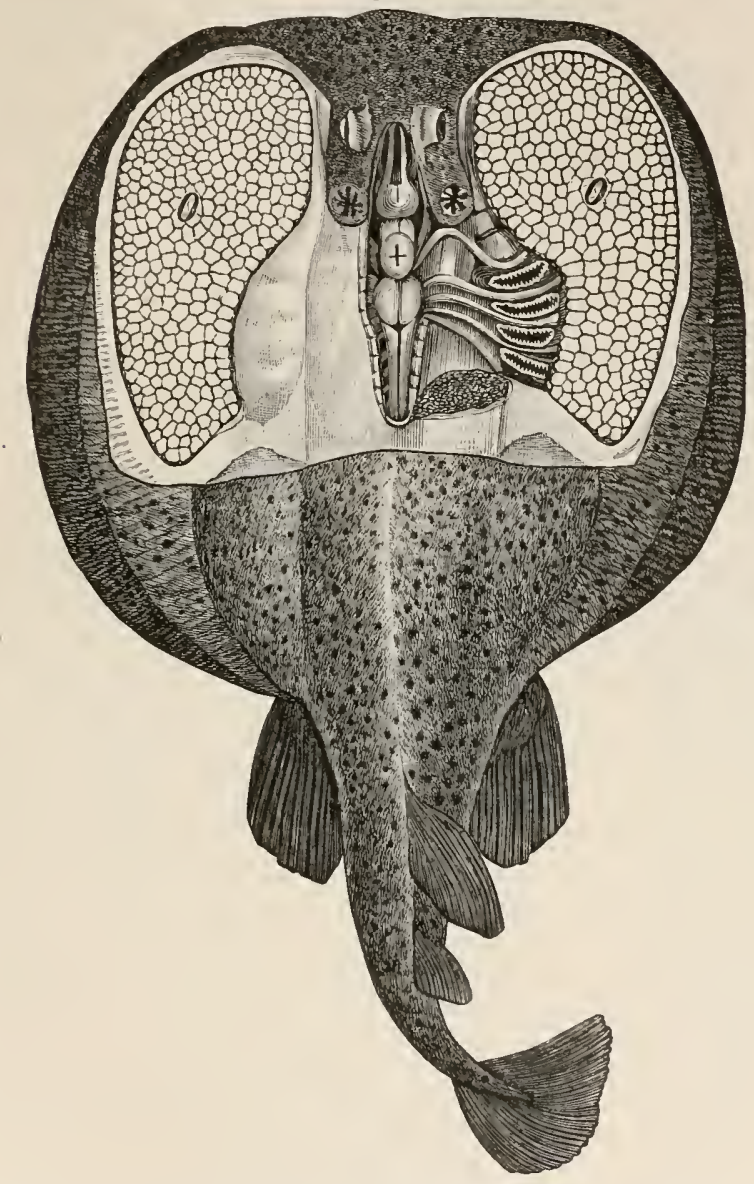

Zitterrochen (Toryedo marmorata).

Rückenfäche, die elektrischen Organe $O$ mit ihren Nerven, das Gehirn nud Ruckeumark freigelegt (nach Fritsch, Die elektrischen Fische, Abt. II, 1890).

Das Organ des Zitteraals (Fig. 36 a u.b) liegt am Schwanzende. Die Säulen desselben sind ebenfalls longitudinal gerichtet 
und von ähnlicher Struktur. Beim Schlage wird das Schwanzende des Organs negativ. Es ist nachgewiesen, daß die Nervenfasern an die Schwanzseite der Querscheiben herantreten. Beim Zitterwels dagegen ist die Art der Nervenverbin-

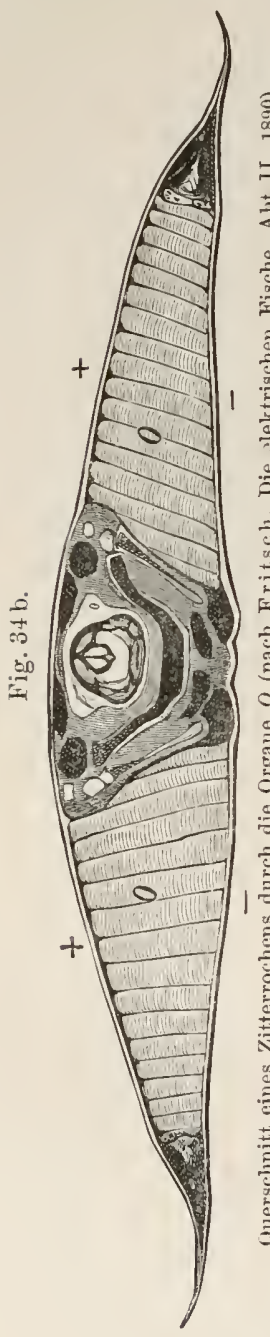
dung noch nicht sicher festgestellt. Sehr eigentümlich ist $\Theta S$, daß der Nerv des Zitterwelses aus einer einzigen dicken Nervenfaser mit vielen Hüllen besteht, die sich im Organ in außerordentlich viele dünne Fasern auflöst.

Die Querscheiben der Säulen, welche durch Bindegewebswände voneinander getrenut sind, hat man als Zellen anzusehen, welche beim Embryo aus denselben Zellen hervorgehen, aus denen sich die quergestreiften Muskelfasern entwickeln. Man hat bei einem Rochen (Raja) die direkte Umbildung quergestreifter Zellen in die des elektrischen Organs beobachtet. Man kanu daher die Elemente des Organs als "elektrische Zellen" bezeichnen. Dieselben bestehen (Fig. 37) aus einer Nervenplatte, in welcher sich die Nervenfasern auflösen, und aus einer dieselbe deckenden Gallertplatte, welche aus einer gallertigen Nasse zusammengesetzt ist. Die Gallertplatte bildet zottenartige Fortsätze, in welche sich die Nervenplatte einsenkt. Diese Zotten sind von einem feinen Stäbchensaum begrenzt. Auf der anderen Seite besitzt die Gallertplatte auch warzenartige Fortsätze, aber ohne einen Stäbchensaum.

Man war anfangs geneigt, das elektrische Organ mit einer Voltaschen Säule zu vergleichen, aber E. du Bois-Reymond hatte schon gezeigt, daß das Organ in der Ruhe keinen oder einen nur sehr schwachen konstanten Strom besitzt. Es entstehen vielmehr bei der Reizung des Organs oder der seiner Nerven kurzdauernde Ströme von beträcht- 


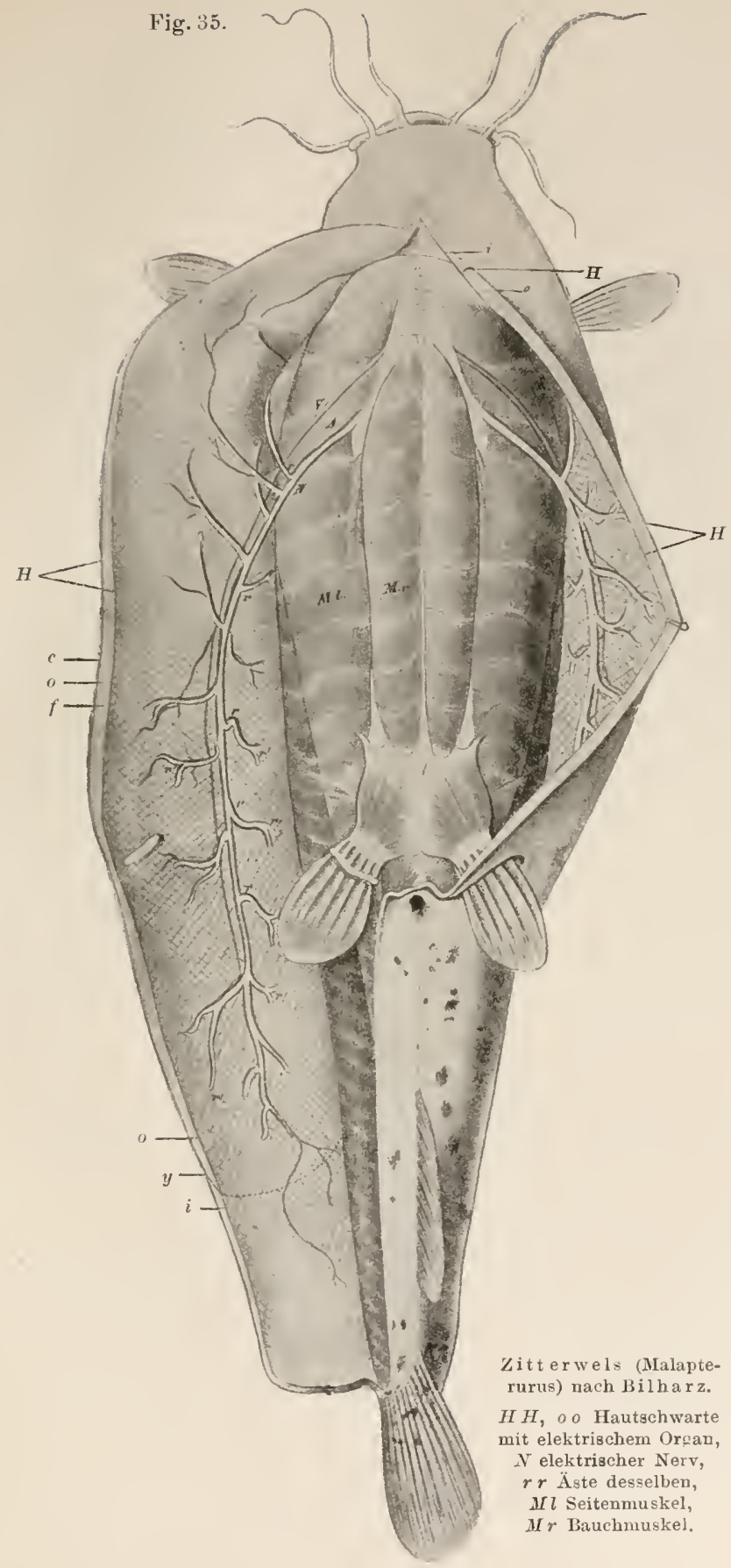


licher Spanuung, aber nicht etwa Wechselströme, wie die eines Induktoriums mit Unterbrecher, sondern Ströme in bestimmter Richtung, wie sich dies schon mit Galvanometer und chemischer Zersetzung von Jodkaliumstärke zu erkennen gab. Marey konnte

Fig. 36 a.

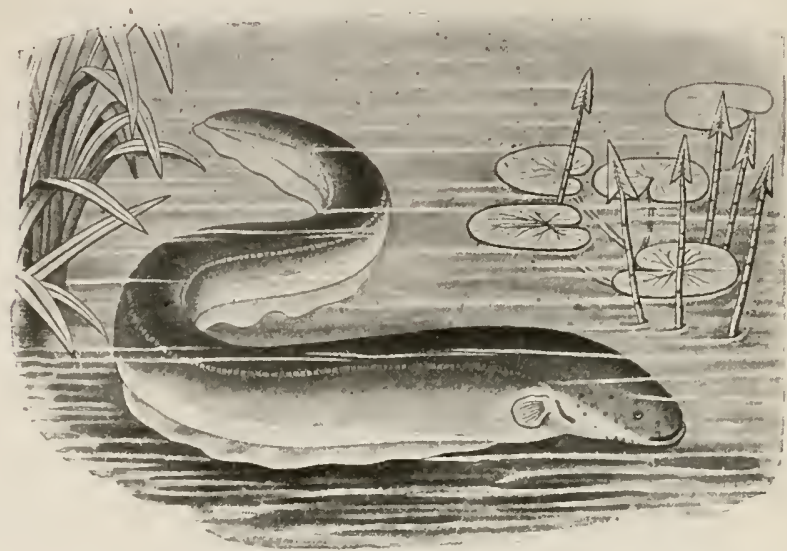

Der Zitteraal (Gymnotus electricus) nach Biedermann, Elektrophysiologie 1895.

an einem Telephon die Schläge hören und aufzeichnen; aber erst von Schoenlein ${ }^{1}$ ) sind genauere Versuche an dem Organ von Torpedo mit dem Rheotom und einem Telephonhebel angestellt worden.

Nach diesen Versuchen erreicht der Strom schnell in 2 bis $3 \sigma$ sein Maximum und ist in 6 bis 86 fast abgelaufen. Bei der Fig. $36 \mathrm{~b}$.

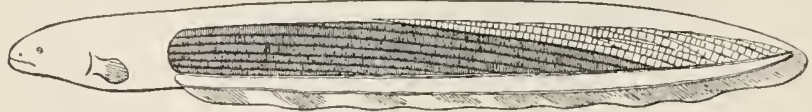

Zitteraal, Langsschnitt (nach Biedermann, ebenda).

Nervenreizung beobachtet man ebenso wie beim Muskel eine Latenz von 3 bis $4 \sigma$, ebenso auch bei direkter Reizung eine etwas kürzere. Im übrigen scheint Dauer und Verlauf je nach der Art der Reizung mit Induktionsschlag oder konstantem Strom

1) Zeitschr. f. Biologie 33, 1896. 
etwas verschieden auszufallen. Die willkürlichen und reflektorischen Entladungen bestehen immer aus einer Reihe von Schlägen,

Fig. 37.
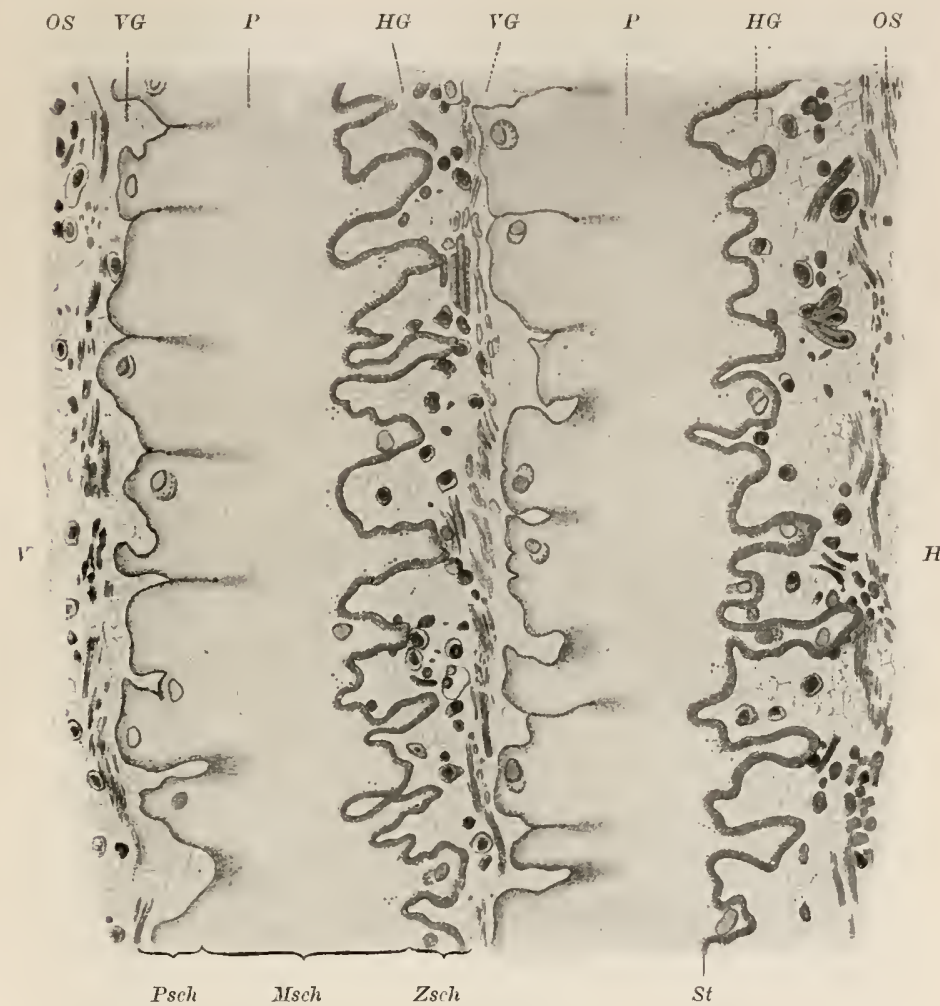

Bau des elektrischen Organs rom Zitteraal nach Ballowitz.

Querschnitt zweier Platten. $\Gamma$ vorn, $H$ hinten. $P$ Gallertplatte. Zsch Zottenschicht, St Stäbchensaum derselben. Msch Mittelschicht. Psch Papillenschicht.

V $G$ u. $H G$ vordere und hintere Grenzschicht, zwischen diesen die Nervenendigungen (Nervenplatte), welche in die Zottenschicht eintreten.

die mit großer Geschwindigkeit, etwa 100 bis 150 in der Sekunde, aufeinander folgen können.

In neuerer Zeit haben Cremer mit einem Saitenelektrometer uud Garten mit einem Saitengalvanometer die Kurve eines Schlages am Torpedo aufgenommen; letztere zeigt Fig. 38. Garten 
- $114-$

Fig. 38.

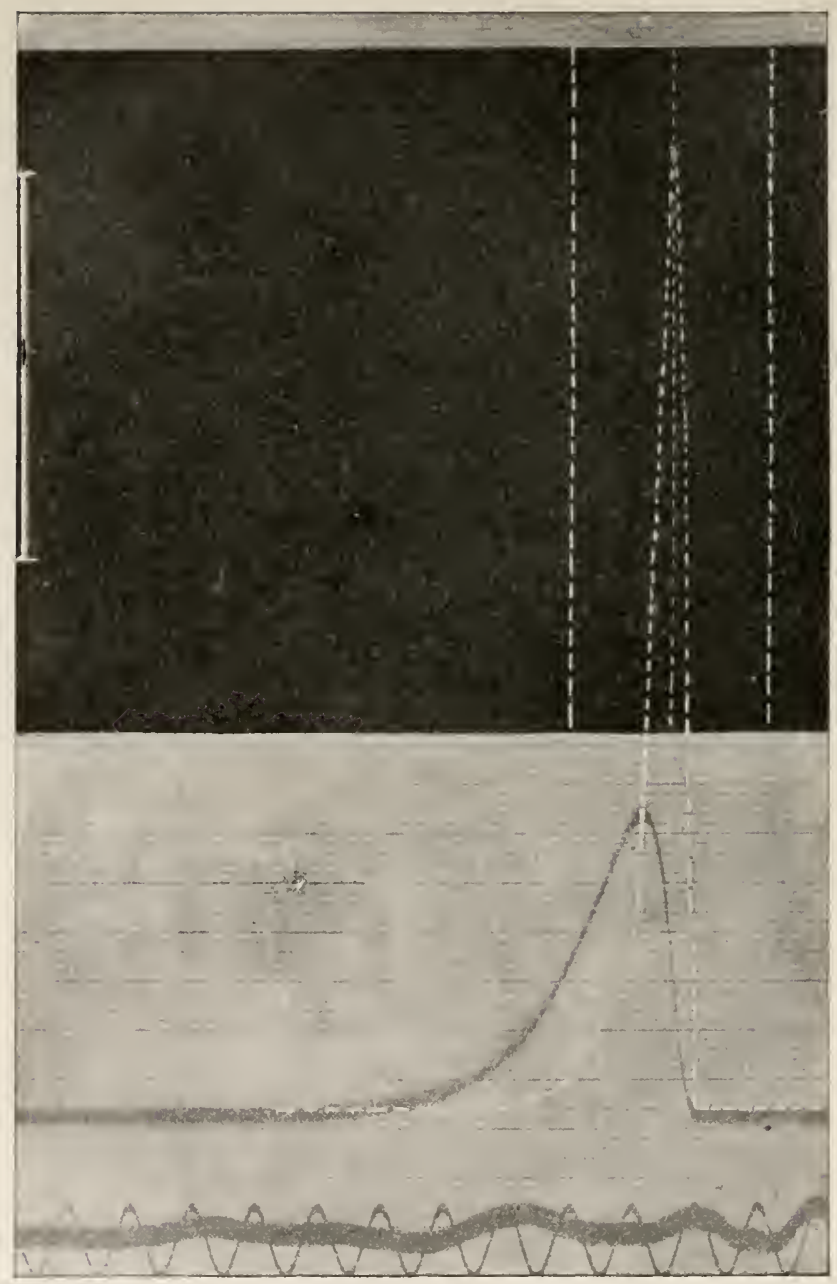

Schlagkurve vom Zitterrochen, Reizung des Nerven mit absteigendem konstanten Strom. (Garten, Handb. d. vergl. Physiol. III.) 
berechnet für den Anstieg $3 \sigma$ und für die ganze Dauer mit langsamem Abfall 20 б; doch war die Temperatur nur 10 bis $11^{\circ} \mathrm{C}$. Die Reizung geschah durch Schließung eines konstanteu Stromes im Nerven. Jedoch fehlen noch genauere Messungen hierüber. Es geht aus den bisherigen Beobachtungen schon hervor, daß der Verlauf des Schlages ganz den Charakter einer negativen Schwankung oder einer Phase des Aktionsstromes vom Muskel besitzt. Der große Unterschied vom Verbalten des unverletzten Muskels besteht aber darin, daß niemals eine Doppelphase auftritt, sondern der Strom nur einphasig ist.

Von Schoenlein ist bereits die Kraft des Schlages bei Torpedo mit dem Rheotom durch Kompensation gemessen worden. Es wurde eine Kette von verschiedener Kraft in entgegengesetzter Richtung in den Kreis eingeschaltet und das Rheotom auf das Maximum des Schlages eingestellt. Es ergab sich, daß die Kraft des Schlages etwa gleich 31 Daniell war. Daraus konnte man die Kraft des einzelnen Elementes der Säulen des Organs, also der einzelnen elektrischen Zelle berechnen, wenn man die Kraft des Schlages durch die mittlere Zahl der hintereinander geschichteten Zellen dividierte. Es fand sich, daß die Kraft eines Elementes etwa von der Größenordnung des Muskelstromes bzw. seiner negativen Schwankung ist, eine bemerkenswerte Tatsache, diefür die Theorie von Bedeutung ist.

Von Cremer sind am lebenden Torpedo reflektorische rhythmische Entladungen mit eineın Saitenelektrometer aufgenommen worden, welche in einem Intervall von $5 \sigma$ dicht aufeinander folgten, also einem Tetanus entsprechen. Auch bei Reizung des Nerven mit konstantem Strom kann ein solcher Tetanus auftreten (Garten). Bei langdauernder Reizung tritt ebenso wie am Muskel bald Ermüdung ein. Auch am lebenden Tiere beobachtet man nach längerer starker Tätigkeit Er'schöpfung. Es wird erzählt, daß die Indianer, um Zitterwelse zu fangen, Pferde in den Fluß treiben und dann die erschöpften Fische leicht fangen können.

Die bisherigen Untersuchungen am Zitterwels und Zitteraal haben zu ganz ähnlichen Ergebnissen geführt, wie die am 'Torpedo, doch konnte man bisher die Versuche noch nicht systematisch durchführen. du Bois-Reymond beobachtete an Zitterwelsen, welche jahrelang in einem erwärmten Bassin gehalten wurden, 
den Verlauf der Schläge. C. Sachs ${ }^{1}$ ) unternahm 1876/77 zur Untersuchung der Zitteraale eine Expedition nach dem Amazonenstrom. Die größere Stärke des Schlages der Zitteraale und Welse erklärt sich zur Genüge aus der größeren Zahl der hintereinander geschalteten Elemente des sehr langen Organs. An herausgeschnittenen Stücken desselben sind freilich die Schläge sehr viel schwächer. Die unverletzten Organe mit ihren Nerven lassen sich nicht so gut isolieren wie am Torpedo, auch sterben sie, da es Organe tropischer Tiere sind, sehr viel schneller ab, als die

Fig. 39.

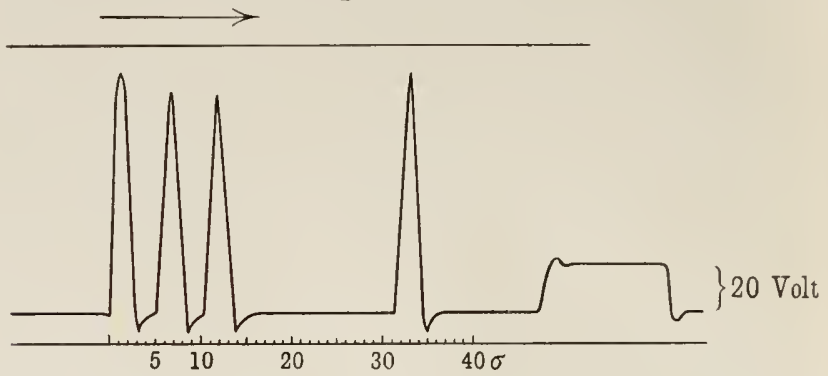

heflexentladung vom Zitterwels nach Cremer (aus Garten, Handb. d, vergl, Physiol.).

des Torpedos. In neuerer Zeit hat Cremer mit dem Saitenelektrometer Schläge des lebenden Zitterwelses aufgenommen, wie Fig. 39 zeigt. Eine Berechnung läßt darauf schließen, daß die Kraft des ganzen Organs beim Zitterwels etwa 450 Volt beträgt. Die Dauer eines Schlages betrug etwa 2,8 б.

Geht man nun auf die Frage ein, welche Art von Kette das elektrische Organ bildet, so muß man nach unseren jetzigen Kenntnissen über die Thermodynamik zunächst prüfen, welchen Temperaturkoeffizienten der Schlag des Organs besitzt, und damn, welche Temperaturänderungen in dem Organ bei der Tätigkeit auftreten.

Solche Untersuchungen unternahmen Bernstein und Tschermak2) an dem elektrischen Organ voin Torpedo. Die

$\left.{ }^{1}\right)$ Beschrieben nach dessen Torte von du Bois-Reymond 1881.

*) Über das thermische Verhalten des elektrischen Organs vou Torpedo. Sitzungsber. d. Berl. Akad. d. Wiss. 1904, S. 301-313. Über die Natur der Kette des elektrischen Organs beim Torpedo. Pflügers Arch. 112, 439-521 (1906). 
Versuche ïber den Einfluß der Temperatur auf die Kraft des Schlages konnten zu einem ganz entscheidenden Resultat nicht führen. Das Organ verhält sich in dieser Beziehung ähnlich wie der Nerv, dessen Rubestrom bei $18^{\circ} \mathrm{C}$ etwa ein Maximum aufweist, der aber doch, wie die genauere Analyse zeigte (s. oben S. 102), sich als Konzentrationsstrom zu erkennen gab. In den Grenzen von 3 bis $18^{\circ} \mathrm{C}$ konnte auch am elektrischen Organ nachgewiesen werden, daß der Temperaturkoeffizient des Schlagstromes ein positiver ist und daf die Kraft des letzteren der absoluten Temperatur annähernd proportional steigt.

Man muß in allen Ketten lebender Organe zwei Temperaturkoeffizienten unterscheiden, den physikalischen, welcher die Voraussetzung involviert, daß die Konstitution der Kette bei wechselnder Temperatur konstant bleibt, und mindestens einen physiologischen Temperaturkoeffizient, welcher von den physikochemischen Änderungen bei wechselnder Temperatur abhängig ist. Der letztere kann positiv oder negativ sein, und da er sich oft aus mehreren Koeffizienten zusammensetzt, aus einem positiven in einen negativen umschlagen oder umgekehrt. Dadurch kann der physikalische Koeffizient mehr oder weniger verdeckt werden.

Beim elektrischen Organ müssen wir daher einen physiologischen Temperaturkoeffizienten annehmen, welcher die liraft des Schlages von etwa $18^{\circ} \mathrm{C}$ ab erheblich verringert. Gehen wir von der Membrantheorie aus, so können wir uns diese Wirkung und die Veränderung der Membran der elektrischen Zellen, welche dieselbe mit steigender Temperatur erleidet, wohl erklären. Bis $18^{\circ} \mathrm{C}$ sind indes diese Veränderungen nicht so bedeutend, um den physikalischen Temperaturkoeffizienten zu maskieren, wie folgende Tabelle zeigt:

\begin{tabular}{|c|c|c|c|}
\hline $\mathrm{N}_{\mathrm{r}}$ & $T$ & $\begin{array}{c}E \\
\text { beob. }\end{array}$ & $\begin{array}{l}E \\
\text { ber. }\end{array}$ \\
\hline 1 & 276 & 185,0 & - \\
\hline 2 & 279 & 188,75 & 187,0 \\
\hline 3 & 282 & 192,0 & 189,0 \\
\hline 4 & 285 & 194,5 & 191,0 \\
\hline 5 & 288 & 197,0 & 193,0 \\
\hline 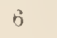 & 291 & 197,25 & 195,0 \\
\hline
\end{tabular}


$T$ bedeutet wiederum die absolute Temperatur, $E$ die beobachteten und berechueten Ausschläge des Galvanometers. Die letzteren wurden den elektromotorischen Kräften proportional sein, wenn der Verlauf der Schläge in einauder ganz ähnlichen Kurven erfolgte, was allerdings nicht genau der Fall ist. Es ist vielmehr anzunehmen, daß bei niederer Temperatur die Dauer des Schlages zunimmt. Dieser Fehler würde aber zugunsten des obigen Resultates sprechen, da mit zunehmender Temperatur die effektiven Kräfte ${ }^{1}$ ) kleiner sein müßten als die beobachteten.

Genauere Resultate würde man nun mit den graphischen Elektrometern erhalten, vorausgesetzt, daß man die Kurven gut analysieren könnte. Leider liegen hierüber nur einige Versuche von Garten ${ }^{2}$ ) (und Koike) vor, welche am lebenden Zitterwels bei 36 und $34^{\circ} \mathrm{C}$ angestellt sind. Bei $16^{\circ} \mathrm{C}$ ergab sich eine Kraft von 68 Volt, bei $34^{\circ} \mathrm{C}$ eine Kraft von 37,5 Volt. Es ergab sich also auch hier über mittlere Temperatur von etwa $18^{\circ} \mathrm{C}$ bis über $30^{\circ} \mathrm{C}$ hinaus eine erhebliche Abnahme der Kraft, wie in den Versuchen von Bernstein und Tschermak an dem isolierten Organ $\nabla$ om Torpedo. Versuche, in denen die Temperatur zwischen 0 und $18^{\circ} \mathrm{C}$ schwankte, sind noch nicht veröffentlicht. Versuche am lebenden Tiere werden sich aber zur Entscheidung der vorliegenden Frage nicht gut eignen, da der Temperaturwechsel noch anderweitige unübersehbare Einflüsse auf das lebende Tier haben muß, welche auf das Organ einwirken werden. Erfolgt der Temperaturwechsel innerbalb längerer Zeiträume, so werden Anpassungen des Tieres an die verschiedenen Temperaturen eintreten, welche den Ernährungszustand der Organe verändern. Nur Versuche am isolierten Organ mit schnell wechselnden Temperaturen können entscheidende Resultate liefern.

Die Temperatur hat im allgemeinen einen ähnlichen physiologischen Einfluß auf die Reizbarkeit der elektrischen Organe wie auf Nerven und Muskeln. Die Reizbarkeit steigt von $0^{\prime \prime}$ bis zu

I) Bei kurzdauernden Strömen sind die Ausschläge des Galvanometers $A=k \cdot \int_{0}^{t_{1}} i d t=k \cdot i_{1} t_{1}$, wo $t_{1}$ die Gesamtdauer, $i_{1}$ die effektive Stromintensität ${ }^{\text {und }} e_{1}=\frac{i_{1}}{w}$ die effektive Kraft ist.

2) Handb. d. vergl. Physiol. 3, 200. 
einer mittleren Temperatur von etwa $18^{\circ} \mathrm{C}$ und nimmt dann bei weiterer Steigerung ab.

Es gibt aber noch einen anderen Weg, die Natur der Kette des elektrischen Organs zu ermitteln. Bernstein und Tschermak notersuchten, welche Temperaturänderungen das Organ bei seiner Tätigkeit erleidet. Wir erinnern uns (s. oben S. 25, 29), daß Konzentrationsketten sich bei der Stromerzeugung abkühlen, und daß die Stromenergie aus der Wärme der Umgebung entnommen wird, daß dagegen chemische Ketten, wie die galvanischen, sich meistens dabei erwärmen, bzw. wie die Daniellsche nahezu konstante Temperatur behalten, und daß bei diesen die ganze bzw. ein großer Teil der Stromenergie aus der chemischen Energie entsteht.

Gehen wir von der obigen Formel (2) aus:

$$
E=Q+T \cdot \frac{d E}{d T}
$$

in welcher $E$ die Kraft, $Q$ die chemische Wärme und $T$ die absolute Temperatur bedeutet, so kann der Temperaturkoeffizient $\frac{d E}{d T}$ größer oder kleiner als Null sein. Ist $Q>E$, so ist $\frac{d E}{d T}$ negativ, wenn $Q<E$ ist, so ist $\frac{d E}{d T}$ positiv, und wenn $Q=E$ ist, so ist $\frac{d E}{d T}=0$. Wenn nun ferner $Q=0$ ist, wie in den Konzentrationsketten, so ist $E=T \cdot \frac{d E}{d T}$. Die Ketten, bei clenen $Q>E$ ist, haben wir exotherme Ketten genannt; sie erzeugen bei der Tätigkeit außer der elektrischen Energie noch Wärme, welche nach außen abgegeben wird; sie erwärmen sich. Diejenigen, bei denen $Q<E$ ist, haben wir endotherme genannt; sie kühlen sich $a b$ und nehmen Wärme aus der Umgebung auf. Einen Aufschluß über die Natur der Kette erhält man daher, wenn man nach den Methoden von Braun, Jahn und anderen (siehe oben S. 28) eine thermodynamische Untersuchung mit ihr vornimmt. Man setzt die Kette in ein Kalorimeter, mißt die Wärmemenge, welche dieselbe in einer gewissen Zeit während der Stromerzengung an das Kalorimeter abgibt oder aus demselben aufnimmt, und man mißt ferner die elektrische Energie, welche die Kette in einem nach außen geleiteteu Stromkreis erzengt. Ist dann $Q$ die chemische Strom- 
wärme der Kette, $C$ die Wärme, welche an das Kalorimeter abgegeben oder von ihm aufgenommen wurde, und $S_{e}$ die im äußeren Stromkreis durch die elektrische Energie gewonnene Wärme, so können wir für alle Fälle die Gleichung ${ }^{1}$ ) aufstellen :

$$
Q=C+S_{e}
$$

Ist $C$ positiv, so ist die liette eine galvanische, exotherme, ist $C$ negativ, so ist die Kette eine endotherme. Ist in letzterem Falle $C+S_{e}=()$, so ist sie eine Konzentrationskette $\left.{ }^{2}\right)$.

Wenden wir diese Betrachtung auf das elektrische Organ an, so haben wir zu bedenken, daß dasselbe in rubendem Zustande gar keine elektromotorische Kraft zeigt, daß es sich also anders verhält, wie eine ungeschlossene physikalische Kette. Erst wenn' eine Reizung stattfindet, verwandelt es sich in eine stromgebende liette. Diese Umwandlung kann nur dadurch geschehen, daß eine Änderung in der chemischen Konstitution eintritt, und diese mußs mit einer Wärmetönung verbunden sein. Außer der chemischen Stromwärme $Q$ müssen wir in solchem Falle daher eine "Umwandlungswärne" IT annehmeu, und die Gleichnng für das Urgan wird also lauten:

$$
U+Q=C+S_{e}
$$

Eine derartige Umwandlung könnte in einer Kette in verschiedenartiger Weise erfolgen. Denken wir uns z. B. ein galvanisches Element, in welchem statt der Tonzelle ein Glasgefäß eingesetzt ist, so würde es keinen Strom liefern; wenn aber durch einen chemischen Prozeß sich das Glas in einen porösen Körper verwandelte, so würde ein Strom entstehen. Allerdings müßten wir auch wieder eine Rückverwandlung annehmen, und in diesem Falle würde die Rückverwandlung gerade so viel Wärme (positiv oder negativ) erfordern als die Verwandlung, und $U$ würde fortfallen. Oder denke man sich eine Konzentrationszelle, in welcher zuerst die Konzentration auf beiden Seiten dieselbe, also die Kraft gleich Null sei, und nun entstehe durch einen chemischen Prozeß auf einer Seite ein Überschuß eines Elektrolyten, so entsteht ein Strom, der so lange dauert, bis dieser Überschuß wieder auf irgend eine Weise beseitigt ist. Da es sich in einem lebenden

1) Nach Formel S. 28 mit veränderter Bezeichnung.

2) Es gibt auch galvanische, endotherme Ketten, wenn $C$ negativ und $C+S_{e}$ positiv ist (s. S. 28). 
Organ wohl nur nu ein organisches lilektrolyt handeln kann, das durch Spaltung und Oxydation entsteht, so müßte dabei merkliche Wärme gebildet werdeu, wie dies im Muskel bei der Kontraktion der Fall ist. Dies wäre eine an die Alterationstheorie sich anschließende Vorstellung. Die Rückverwandlung würde aber viel weniger Wärmeenergie absorbieren, als entstehen würde, wie dies auch beim Muskel der Fall ist, denn die elektrolytischen Produkte könnten nicht wieder zur Restitution der Zellsubstanz verwendet werden. In solchem Falle hätten wir im elektrischen Organ bei der Tätigkeit eine merkliche Wärmebildung zu erwarten.

Gehen wir nun zur Anwendung der Membrantheorie über, welche sich bei der Erklärung der Muskel- und Nervenströme und ihrer Tätigkeitsschwankungen hinreichend bewährt hat. Wir stellen uns nach, dieser vor, daß die elektrische Zelle auf beiden Seiten ron einer semipermeabeln Plasmamembran umbüllt ist und daß sie mit Fig. 40 .
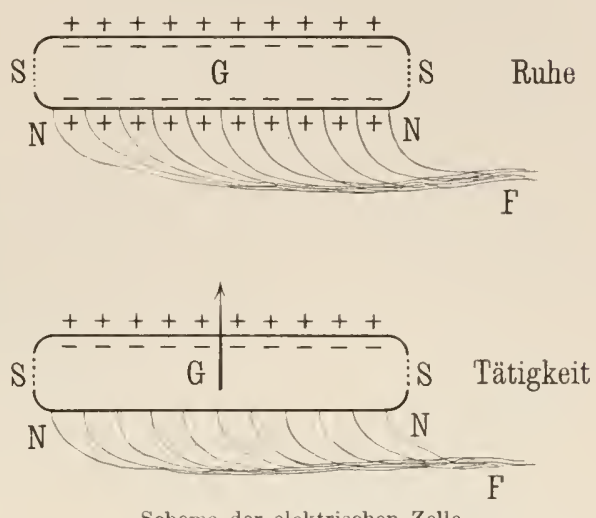

Schema der elektrischen Zelle.

der Lösung eines Elektrolyteri geladen sei. Dieser Elektrolytbehälter sei die Gallertplatte, an welche sich anf einer Seite die Nervenplatte anlegt (s. Fig. 37).

In Fig. 40 sei nach dem mikroskopischen Bilde von Fig. 37 die elektrische Zelle schematisch dargestellt. $G$ sei die Gallertplatte, welche von einer Plasmamembran umbüılt ist. An eine Seite derselben legt sich die Nervenplatte $N N^{\top}$ an, welche durch Auflösung der Faser $F$ in feine Endigungen entsteht. Nehmen wir an, daß die Plasmamembran auf beiden Seiten für das Kation durchlässig, für das Anion aber nicht oder schwer durchlässig ist, wie es die +- und - Zeichen angeben, so ist die Zelle in der Ruhe stromlos. Findet aber eine Reizung vom Nerven aus statt, so wird die Nervenseite der Plasinamembran auch für das Anion permeabel, die elektrische Doppelschicht der Nervenseite 
verschwindet und die andere Seite erzeugt einen Strom in der Richtung des Pfeiles, wie ihn das Organ gibt.

Es müßte nun diesem Strom ein entgegengesetzter folgen, wie es bei den Aktionsströmen des unverletzten Muskels der Fall ist, wenn sich die Reizung auf die entgegengesetzte Seite der Membran fortpflanzte. Aber entweder ist die Reizleitung durch die Seitenwände $\left.{ }^{1}\right)(S S)$ unterbrochen, oder die Membran ist auf der distalen Seite überhaupt nicht reizbar. In der Tat zeigt die Nervenseite der Membran (siehe Fig. 37), als Zottenschicht bezeichuet, eine Stäbchenstruktur, wäbrend diese auf der anderen Seite, in der Papillenschicht, fehlt. Die Mittelschicht, welche aus der eigentlichen Gallertschicht besteht, enthält wahrscheinlich kein reizbares und reizleitendes Plasma und bildet wohl hauptsächlich den Elektrolytbehälter.

Die Umwandlung, welche beim Schlage erfolgt, besteht hiernach in der Veränderung der Plasmamembran, wie bei der negativen Schwankung der Muskel- und Nervenströme. Die Wärmetönung dieses Prozesses braucht daher nur eine geringe zu sein, wie dies auch am Muskel und Nerven der Fall ist. Die Umwandlungswärme $U$ wäre also nach der Membrantheorie nur eine kleine, und die chemische Stromwärme $Q$ würde Null sein, da der Elektrolyt schon als vorhanden angenommen wird.

Die angestellten Versuche bestätigten diese Voraussetzungen. Die Methode der Untersuchungen war folgende. Zur Messung der Wärmemenge, welche beim Schlage in dem Organ entsteht, konnte ein Eiskalorimeter, wie bei den physikalischen Versuchen von Jahn, nicht angewendet werden, da die Abkühlung auf $0^{\circ}$ dem Organ zu schädlich gewesen wäre. Es wurde daher die auch bisher für den Muskel gebrauchte thermoelektrische Methode benutzt, indem die isolierten Organe in einen wärmedichten Behälter (Pappkasten von doppelten mit Schafwolle ausgefüllten Wänden) gelegt wurden und durch Anlegen einer empfindlichen Thermosäule (meist die Heidenhainsche 15́gliedrige WismutAntimonsäule) die Temperaturänderung mit einem empfindlichen Thermogalvanometer gemessen wurde. Dadurch konnte aus der Nasse des Organs und seiner spezifischen Wärme (im Mittel 0,8694)

I) Ihre Beschaffenheit ist bis jetzt mikroskopisch nicht speziell beschrieben. 
die entsprecheude Wärmemenge berechnet werden. Mit Hilfe eines Sekundenpendels wurde das Organ von seinen Nerven aus durch Induktiousströme 1 Sekunde lang tetanisch gereizt. Die Wärmeenergie der entstehenden Schläge wurde mit einem eigeus konstruierten Luftthermometer bzw. -kalorimeter gemessen. Dasselbe besteht nach dem Muster des Riebschen elektrischen Luftthermometers, wie Fig. 41 angibt, aus einer Kapillarröhre $t$ $(0,61 \mathrm{~mm}$ Durchmesser), welche auf der einen Seite in ein Gefäß $g$, auf der anderen in die elektrische Birne $B$ mit Kohlenfaden übergeht. Wird Gefäß und Röhre mit Flüssigkeit gefüllt (alkoholische Methylenblaulösung), der Hahn a geschlosseu und werden dio Ströme der Organe während 1 Seliunde durch den Kiohlenfaden geleitet, so erhält man vom elektrischen Organ oft Ausscbläge der Flüssigkeitssäule bis über $100 \mathrm{~mm}$. Das Instrument wurde mit Sekundenströmen be-



Lufthermometer zur Messung der Stromwärme des Schlages (Bernstein und Tschermak).

kaunter Intensität nach der Formel für die Stromwärme $q=0,2394 i^{2} \cdot v$ empirisch graduiert, und so konnte die äußere Stromenergie $S_{e}$ der Schläge der Organe berechnet werden ${ }^{1}$ ). Die Anordnung der Versuche ist in Fig. 42 schematisch wiedergegeben. $O$ ist der Kasten, in welchem die Organe eingeschlossen sind. Sie werden von beiden Seiten, oben und unten, mit unpolari-

$\left.{ }^{1}\right)$ In späteren Versuchen wird man gut tun, genaue Kalorimeter zu benutzen, für die äußere Stromenergie ein Eiskalorimeter; für die Organe aber müßte ein Kalorimeter nach dem Prinzip des Eiskalorimeters für mittlere Temperaturen erst erfunden werden (alle bisherigen Wasser- oder Luftkalorimeter sind nicht genau genug). Dann könnte man die Reizung periodisch bis zur Erschöpfung fortsetzen und würde im ganzen größere Wärmewerte gewinnen. 


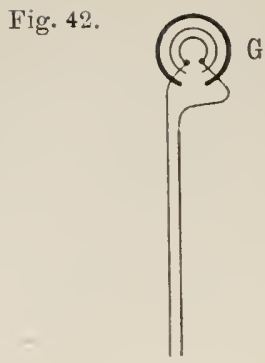

sierbaren Elektruden aus Zinkplatten, Zinksulfat und Kochsalzbauschen zum Schlüssel $S_{1}$ und von da zum Luftthermometer $I, 77$ abgeleitet. Die durch eine Lücke der oberen lilektrode aufgesetzte 'Thermosäule führt durch den Rheostaten $W$ zum Galvanometer $G$. Durch eine Kompensationskette $C$ mit Rheochord kann etwaige Ungleichheit der* Thermosäule auf Null kompensiert werden. $F$ und $S k$ sind Fernrohr und Skala. Die Reizung derNerven geschah durch den Schlittenapparat s, von dessen sekunidärer Spule die Leitung zu dem $\mathrm{Sk}$ Sekundenpendel $I I$ und zu den Reizelektroden aus Platin in $O$ führt. Die Ablenkungen des Galvanometers wurden nach Skalenteilen auf einem Kymographen $K$ mit dem Tastschlüssel $I$ durch ein Signal Deprez $u_{T}$ markiert. Zugleich wurde die Reizdauer durch Signal $M_{R}$ von einem damit isochronen Kontakt $I I$ aus und die Zeit in Sekunden durch ${ } I_{Z}$ verzeichnet. Ein 
Beobachter saß am Fernrohr, der andere an Lufthermoneter. Ein Skalenteil Ablenkung am Galvanometer bedeutete $1 / 10000^{\circ} \mathrm{C}$. Eine feine Gummibaut schützte die Thermosäule vor dem Einbrechen des Organstromes, da die Lackierung nicht ausreichte und sonst arge Täuschungen entstehen können.

Man konnte uun die Versuchsart in dreierlei Weise variieren. Erstens, indem man mit Arbeitsleistung nach außen die Schläge des Organs durch das Lufthermometer leitet. Diese Art entspricht einer Arbeitsleistung des Muskels durch Hebung von Gewichten, welche gehoben bleiben (Arbeitssammlung). Zweitens können wir den Schlüssel $S_{1}$ als Kurzschluß benutzen, so dals fast keine Arbeitsenergie beim Schlage nach außen geleitet wird und der Strom nur im Organ kreist. Wir würden diese Art mit der isometrischen Kontraktion des Muskels vergleichen können, bei welcher er keine äußere mechanische Arbeit leistet. Wir können drittens diesen Fall noch dadurch variieren, daß wir das Organ beim Schlage möglichst isolieren, so daß kein oder nur ein schwacher Strom (durch innere Abgleichungen) zustande kommt.

Im ersten Falle haben wir die Gleichung:

$$
U+Q=C+S_{c},
$$

im zweiten Falle die Gleichung:

$$
U+Q=C
$$

und im dritten Falle die Gleichung:

$$
U=C \text {. }
$$

Es ist zu beachten, daß $Q$ die Stromwärme bedeutet, welche aus dem chemischen Prozeß infolge Schließung einer Kette entsteht. Sie fällt also in letzterem Falle aus, und es bleibt nur die Umwandlungswärme $U$ übrig.

Die Versuche ergaben nun, daß ganz im Gegensatze zum Muskel überhaupt nur sehr geringe Temperaturerhöhungen im elektrischen Organ entstehen. Dieselben erreichen trotz beträchtlicher Leistung meist nicht $2 / 1000$ bis $4 / 1000^{\circ} \mathrm{C}$. In einer größeren Zahl von Beobachtungen lieb sich sogar eine deutliche Abkühlung des ()rgans nachweisen. Hierdurch war es festgestellt, daßdas Organ wie eine endotherme Kette arbeitet. Zur Erzeugung der Stromenergie wird zunächst die Umwandlungswärme $U$ verwendet, und wenn diese nicht ausreicht, so wird Wärme des Organs bzw. der Umgebung in elektrische Energie 
umgesetzt. Ein Wert für eine chemische Stromwärme $Q$ läbt sich nicht erweisen. Dies ist aber der untrügliche Beweis dafür, daf das elektrische Organ eine Konzentrationskette ist ${ }^{1}$ ).

Folgende Tabelle zeigt einige Beispiele für die Abküblungen des Organs in Galvanometerausschlägen, welche bei Zuleitung der Schläge zum Lufthermometer beobachtet wurden:

\begin{tabular}{|c|c|c|c|}
\hline Nr. & $\begin{array}{l}\text { Galvanometer- } \\
\text { ausschlag }\end{array}$ & $\begin{array}{l}\text { Luftthermometer- } \\
\text { ausschlag }\end{array}$ & $\begin{array}{c}\text { Differenz von Organwarme } \\
\text { und innerer Stromwärme } \\
\qquad C-S_{i}\end{array}$ \\
\hline 1 & - 1,0 Sek. & $3,16 \mathrm{~mm}$ & $-0,0217 \mathrm{~g}-\mathrm{Cal}$ \\
\hline 2 & $-4,2 \quad n$ & 92,21 n & $-0,2548 \quad n$, \\
\hline 3 & $-2,2 \quad$ & 1,05 , & $-0,0387$ \\
\hline 4 & $-0,3$ & 2,09 n & $-0,0100$ \\
\hline 5 & $-0,1 \quad$ & 0,25 & $-0,0029$ \\
\hline
\end{tabular}

Man sieht, daß mit der Stärke der Schläge die Abkühlung des Organs zunimmt. In Nr. 2 ist bei der sehr kräftigen Entladung die Abkühlung am stärksten gewesen. Gleich nach dieser Beobachtung wurde eine Reizung unter Kurzschluß im Schlüssel $S_{1}$ vorgenommen, wobei die ganze elektrische Stromenergie das Organ erwärmte und eine Galvanometerablenkung $+14,2 \mathrm{Sek}=0,0015^{\circ} \mathrm{C}$ entstand. Die Rubrik $C-S_{i}$ gibt die Wärmemenge an, welche das Organ aufgenommen hat, also den Grad der Endothermie. Hierbei ist von der Kalorimeterwärme $C$ die innere Joulesche Stromwärme $S_{i}$ abgezogen, weiche beim Durchströmen des Organs entsteht. Es kommt auch manchmal vor, daß negative und positive Ausschläge des Galvanometers miteinander wechseln, und zwar gehen die negativen (Abkühlung) den positiven (Erwärmung) meistens voraus. Die Umwandlungswärme ist dann anfangs zu

1) Es ist mir unverständlich, wie Garten (Verhandl. d. deutsch. Naturf. Ges. 1911, I, S. 171) diese Abkühlungen beim Schlage des Organs als Folge einer Assimilation deuten kann. Eine Assimilation kann doch erst nach einer vorangegangenen Dissimilation einsetzen und erfolgt viel langsamer als diese. Die Erwärnıung durch Dissimilation muß daher zuerst immer überwiegen. Selbst beim Muskel, der doch einen viel stärkeren Stoffwechsel hat als das elektrische Organ, kamn man bisher eine Abkühlung durch Assimilation nach der Reizung nicht nachweisen. 
klein, um die elektrische Energie, die mit großer Kraft einsetzt, zu decken.

Die starke Endothermie des elektrischen Organs ist ein in der Physiologie des tierischen Organismus bisher einzig dastehendes Faktum. Reduzieren wir in dem obigen Falle Nr.2, in welchem die Organe $280 \mathrm{~g}$ wogen, ibre Größe auf $100 \mathrm{~g}$, so erhalten wir einen Wert von $0,093 \mathrm{~g}$-Cal in 1 Sekunde. Vergleichen wir diesen mit der Endothermie der chlorophyllhaltigen Pflanze bei der Photosynthese ${ }^{1}$ ), so erhält man für $1 \mathrm{qcm}$ Blatt von Helianthus annuus von 0,02 g Gewicht 0,0033 g-cal/Min.


das elektrische Organ. In diesem Falle ist es strahlende Energie der Sonne, welche sich in chemische Energie umsetzt, das elektrische Organ dagegen verwandelt ungeordnete Wärmebewegung vernittelst osmotischer Kraft unter Verbrauch von Elektrolyt in elektrische Energie.

Es wurde in vielen Versuchen zwischen äußerer Arbeit im Lufthermometer und Kurzschluß, zwischen Arbeit und Isolation der Organe, und zwischen Kurzschluß und Isolation abgewechselt. Es ergab sich, daß bei Kurzschluß und Isolation niemals Abkühlungen des Organs auftreten. Dies folgt aus den Formeln $U+Q=C$ und $U=C$. Bei Kurzschluß bleibt die Joulesche Stromwärme im Organ, und bei Isolation entsteht sie nicht. Da aber auch ein Unterschied zwischen Kurzsehluß und Isolation in der Erwärmung nicht hervortrat, so stimmen die Resultate sehr gut mit der Annahme, daß $Q$ überhaupt Null ist. Wir können daher die obigen Formeln reduzieren auf erstens bei äußerer Arbeit:

$$
U=C+S_{c},
$$

zweitens bei Kurzschluß:

drittens bei Isolation:

$$
U_{k}=C_{k},
$$

$$
U_{i}=C_{i} \text {. }
$$

Ob $U_{k}$ immer mit $U_{i}$ unter gleichen Bedingungen übereinstimmt, ist fraglich. Es könnte durch den Schlag selbst $U_{k}$ größer werden als $U_{i}$.

Wie alle Organe, unterliegt auch das elektrische in hohem Grade der Ermüdung. Dazu gesellt sich beim Versuch am iso-

1) Horace T. Brown, s. Naturw. Rdsch. 1895, S. 354. 
lierten Organ das Absterben desselben mit der Zeit. Dadurch werden die Versuche in ihrer Ausführung mühsam und man muß sie in großer Zahl, wie es geschah, vornehmen. In Fig. 43 sind die Ausschläge des Galvanometers und Luftthermometers mit der Zeit als Abszisse in Kurven dargestellt. Man sieht, daß die erste

Fig. 43.

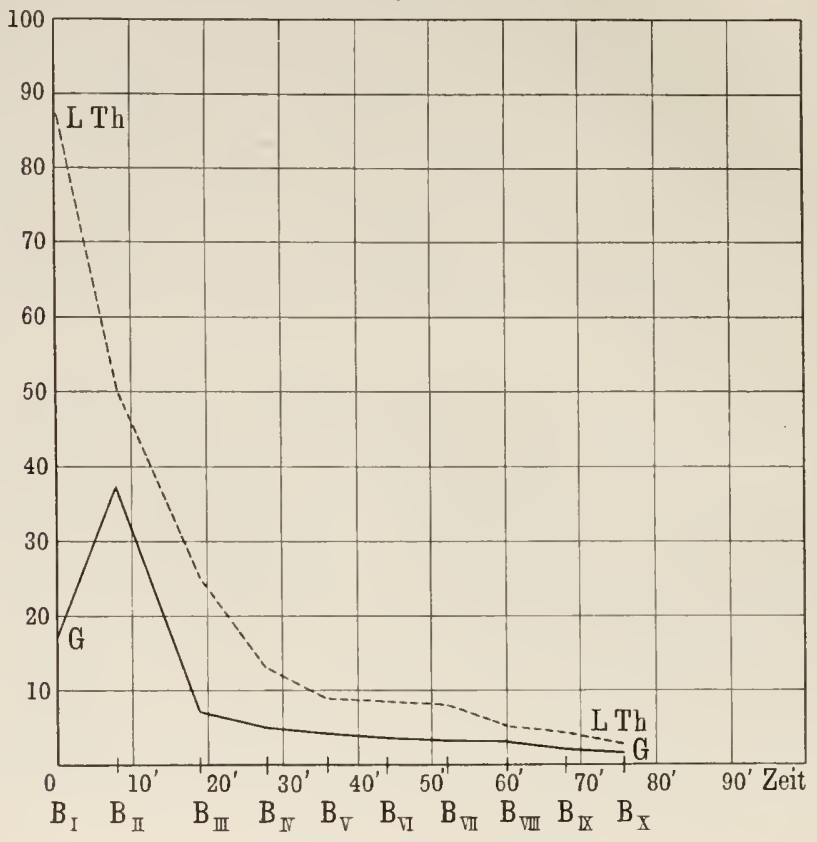

Kurve GG Frwärmuugen des Organs; Kurve $L T h$ Euergie der Schläge, am Luftthermometer gemessen, bei den Beobachtungen; $B_{1}$ bis $B_{X}$ Zeitabszisse in Minuten (Bernstein und Tschermak).

Reizung bei $0^{\prime}$ die stärksten Schläge gab, aber nur geringe Erwärmung im Organ, die zweite nach $8^{\prime}$ schon geringere Schläge, aber viel größere Erwärmung. Dann nahmen Erwärmung und Schlagenergie kontinuierlich bis zu Ende nach 70 Minuten ab.

Die Ermüdung wird durch zweierlei Vorgänge herbeigeführt, erstens durch den Verlust an Elektrolyt, da während des Schlages eine gewisse Menge desselben durch die Membran der Nervenseite nach au@en tritt, und zweitens durch Veränderung der Membran, 
in welcher die Umwandlung erfolgt; denn diese erleidet wie jede plasmatische Masse durch erhöhten Stoffwechsel eine Herabsetzung der Reizbarkeit. Nach stärkerer Tätigkeit bleibt ein Reststrom des Organs zurück, der erst allmählich schwindet, was schon du Bois-Reymond am Zitterwels beobachtet hatte. Derselbe erklärt sich aus dem Ermüdungszustand der Membran, die einen Rest von Permeabilität zurückbehält. WVahrscheinlich erholt sich zunächst die Nembran durch Vorgänge der Ernährung. Langsamer erfolgt vermutlich der Ersatz des Elektrolyten, denn die lebenden Fische sind nach starker Reizung stundenlang erschöpft. Außerdem nehmen wohl auch die Nervenzentren und die Nervenplatte, weniger die Nervenstämme, an der Ermüdung teil.

Auf welche Weise der Ersatz des Elektrolyten erfolgt, bedarf besonderer Erforschung. Daß die Osmose hierzu nicht ausreicht, ist klar, da die Konzentration in der elektrischen Zelle eine größere werden muß als außen. Wenn man daher, wie für Muskel und Nerv, die Salze als wirksame Elektrolyte ansieht, so müßte man vermuten, dafo diese in einer organischen Bindung durch Assimilation in die elektrische Zelle bineingelangen und darin dann frei werden. Die bisherigen chemischen Untersuchungeu des Organs geben noch wenig Anhaltspunkte hierfür. Aber sehr bemerkenswert scheint es mir, dab das elektrische Organ das wasserreichste der Wirbeltiere ist. Th. Weyl ${ }^{1}$ ) gibt an, daß es im Mittel bei Torpedo 88,82 Proz. Wasser enthält. Ich vermute, daß dieser hohe Wassergehalt der Gallertplatte, dem Elektrolytbehälter zukommt. Trotzdem ist der Aschegehalt des frischen Organs ein ziemlich hoher, etwa 1,67 Proz., was dafür spricht, daß die Salze eine besondere Rolle spielen. Unter diesen zeichnen sich Chloride und Phosphate durch größere Menge aus. Weyl fand in der Asche 12,4 bis 17.8 Proz. $\mathrm{P}_{2} \mathrm{O}_{5}$ und 21,38 bis 35,1 Proz. Cl, und auffallenderweise einen viel höheren $\mathrm{Na}$ - als K-Gehalt vor, was er auf den Reichtum des Meerwassers an NaSalzen schiebt. Er gibt ferner an, daß das längere Zeit durch elektrische Ströme direkt gereizte Organ eine meist nur geringe Vermehrung der löslichen $\mathrm{P}_{2} \mathrm{O}_{5}$ zeigte ${ }^{2}$ ). Dies könnte wohl die

1) Zeitschr. f. physiol. Chem. 7, 543 (1883).

$\left.{ }^{2}\right)$ D u Bois-Reymond, Arch. f. Physiol. 1884, S. 319. - Carl Sachs (1. c.) gibt an, daß beim Absterben und bei längerer Reizung der Organe des Zitteraals eine saure Reaktion auftrat. 
Folge des Stoffwechsels der organischen Phosphatide (Lecithine, Nukleine) des Organs sein. Dagegen ist der Aschegehalt der ruhenden Organe größer als der der gereizten; ferner ist der Alkoholextrakt der gereizten Organe geringer als der der ruhenden, während es sich beim Muskel nach Helmholtz umgekehrt verhält. Leider stehen neuere Untersuchungen über diesen Gegenstand nicht zu Gebote. Angaben über die Cliemie der elektrischen Organe der Süßwasserfische fehlen gänzlich.

Aus diesen Untersuchungen kann man über die Natur der wirksamen Elektrolyte in der Konzentrationskette des elektrischen Organs noch nichts Sicheres entnehmen. Aber man dürfte die Vermutung aussprechen, daß die Phosphate und vielleicht die Glyzeriuphosphorsäure, welche bei der Spaltung der Lecithine entsteht, solche Elektrolyte seien. Milchsäure konnte Weyl in dem elektrischen Organ nicht nachweisen. Man wird auch zwischen dem Stoffwechsel der Nervenplatte und dem der Gallertplatte wohl zu unterscheiden haben.

Man hat vielfach die Frage diskutiert, ob die Elemente des Organs als Derivate von Muskelfasern oder als Nervenendapparate anzusehen sind. Da sie aus gleichartigen homologen Embryonalzellen entstehen und die kontraktile Substanz bei der Entwickelung entweder gar nicht entsteht oder sich rückbildet, so erübrigt sich diese Fragestellung. Durch Kurare lassen sich die Nerveneuden der elektrischen Organe nicht so schnell lähmen wie die der Muskeln; erst große Dosen wirken (Steiner). Durch Strychnin kann man volı den Zentren aus Tetanus der Organe herbeiführen.

\author{
Siebentes Kapitel.
}

\title{
Tnnere Polarisation und elektrische Reizung.
}

Die wichtigsten Erscheinungen der inneren Polarisation an Nerven und Muskeln sind schon von du Bois-Reymond gefunden worden. Er nannte den Zustand, in welchen der Nerv durch einen konstanten Strom versetzt wird, "Elektrotonus". Später 
fand er auch, daß eine innere Polarisation eintritt, welche der gewöhnlichen Polarisation ähulich ist, glaubte aber, daß beide Vorgänge verschieden seien. Das ist nicht der Fall. Ma teucci und L. Hermann erklärten vielnehr den Elektrotonus als eine Folge der inneren Polarisation.

Die innere Polarisation ist wach Versuchen von L. Hermann am stärksten, wenn man den konstanten Strom quer durch Muskeln oder Nerven leitet. Man konstatiert in diesem Falle, daß unmittelbar nach der Öffnung dieses Stromes ein entgegengesetzter Polarisationsstrom auftritt, der sehr schnell auf Null absinkt. Der Ablauf dieser Depolarisation läßt sich mit dem Rheotom gut beobachten (Bernstein). In getöteten Organen ist diese Polarisation nicht vorhanden. Dieselbe entsteht in jeder einzelnen Faser an der Anoden - und Kathodenseite derselben, und ihre Gesamtkraft nimmt daher cet. par. mit der Zahl der querdurchströmten Faser'n zu.

An einem Nerven beobachtet man daher auch bei longitudinaler Durchleitung eines konstanten

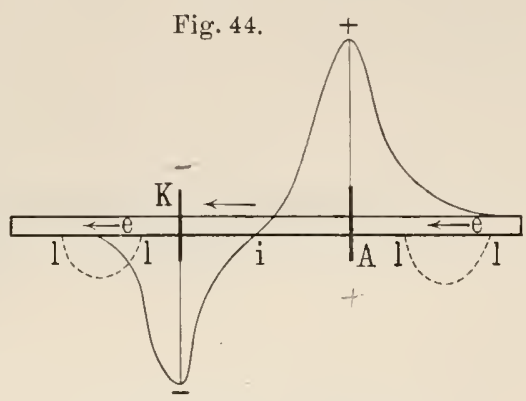

Innere Polarisation des Nerven und elektrotonische Ströme.

Stromes durch eine Strecke desselben, daß an der Anode und Kathode eine innere Polarisation auftritt. Die äußere Polarisation muß durch unpolarisierbare Elektroden (s. oben S. 3 u. 4) vermieden werden. Seien in Fig. $44 A$ und $K$ die Elektroden, so entsteht nicht nur in der intrapolaren Strecke innere Polarisation, sondern sie breitet sich auch auf eine gewisse Entfernung in die extrapolare Strecke hin aus. Die aufgetragene Kurve gibt die Stärke dieser Polarisation an, in $A$ die der anodischen, in $K$ die der kathodischen Polarisation. Leitet man von $l 7$ auf beiden Seiten ab, so erhält man Ströme in der Richtung von $e$, welche im Nerven dieselbe Richtung wie der polarisierende Strom haben. Diesen Zustand nannte du Bois Elektrotonus, die extrapolaren Ströme die elektrotonischen Ströme. Es ist klar, daß sich diese elektrotonischen Ströme mit den Nervenströmen 
kombinieren werden. Leitet man auf beiden Seiten von Längsund Querschnitt ab, so verstärkt sich der Strom auf der Anodenseite, auf der Kathodenseite wird er geschwächt, oder umgekehrt. Unterbindung oder Abtötung des Nerven an einer Stelle hebt die Ausbreitung dieser Polarisation über dieselbe auf.

L. H ermann verglich die Nervenfaser mit einem sogenannten Kernleitermodell. Ein Platindraht ist durch eine au beiden Enden zugekorkte Glasröhre gezogen, welche mit verdünnter Schwefelsäure gefüllt ist und mehrere Seitenröhren besitzt, durch die man Strome zuleiten und ableiten kann. Leitet man durch eine Strecke einen polarisierenden Strom, so kann man extrapolare Ströme ableiten infolge der inneren Polarisation, welche an der Oberfläche des Platindrahtes entsteht. Diese Ströme haben die gleiche Richtung im Keruleiter, wie der polarisierende und verhalten sich

Fig. 45.

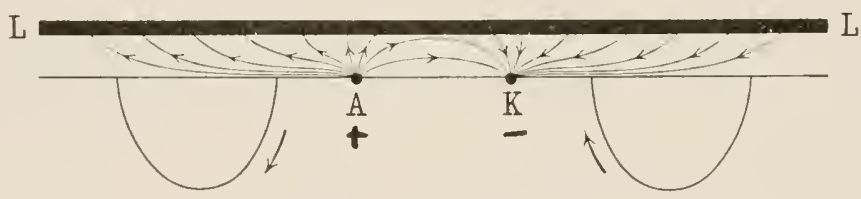

Polarisation des Kernleiters.

wie die elektrotonischen Ströme des Nerven. In Fig. 45 sieht man, wie sich der polarisierende Strom an den Elektroden $A$ und $K$ in der Flüssigkeit in Fäden ausbreitet, um zum Kernleiter $L L$ zu gelangen. Im Nerven bilden die Hüllen und Bindegewebselemente gute Leiter für diese Stromfäden; im Muskel breiten sich diese Ströme nicht so weit aus, da die Hüllen gegen die Fasermassen weniger entwickelt sind. Es handelt sich nicht um gewöhnliche Stromzweige körperlicher Leitér, denn sie haben auch auf der den Elektroden gegenüberliegenden Seite des Nerven dieselbe Richtung und hören auf nach Durchtremnung des Nerven und Aneinanderlegen der Querschnitte. Mit der Stärke des polarisierenden Stromes wachsen die inneren Polarisationen und breiten sich weiter aus. Die anodische Polarisation ist im Nerven eine stärkere als die kathodische, Unterschiede, welche auch bei der physikalischen Polarisation auftreten. 
Wir sind nun imstande, die innere Polarisation nach der Membrantheorie zu deuten und weitere Folgerungen daraus zu gewinnen.

Es sei in Fig. $46 B B$ eine Faser mit der semipermeablen und einer elektrischen Doppelschicht bekleideten Membran. Werden die Elektroden des polarisierenden Stromes in $A$ und $K$ angelegt, so wandern die Ionen der Elektrolyte innerhalb und außerhalb der Faser zur Membran und verhalten sich dort gegen die schon vorhandenen Ionen in verschiedener Weise. Im Bereich der Anode $A$ addieren sich die negativen Ionen des inneren Elektro-

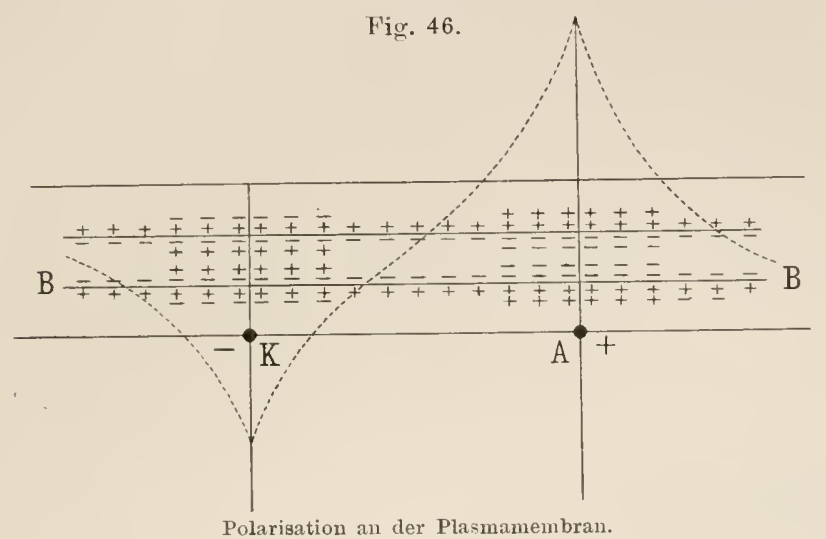

lyten zu den schon vorhandenen, da sie nicht durchtreten können und erhöhen durch Polarisation das Membranpotential. Im Bereich der Kathode verbinden sich die positiven Ionen des inneren Elektrolyten mit den vorhandenen negativen und vermindern daher das Membranpotential daselbst. Der äußere Elektrolyt sendet seine positiven Ionen gegen die Membran im Bereich der Anode, und von diesen können wir annehmen, daß sie ebenfalls von der Membran nicht durchgelassen werden, sondern sich zu den vorhandenen addieren und das Membranpotential erhöhen. Diese Ionen bestehen ja (s. oben S. 99) wohl hauptsächlich aus $\mathrm{Na}$-Ionen, von denen wir wissen, daß sie in den Fasern fast gänzlich fehlen. Die negativen Ionen des äußeren Elektrolyten wandern aber zur Membran im Bereich der Kathode, verbinden sich daselbst mit den vorhandenen positiven Ionen und vermindern 
demnach das Membranpotential. Die +- und - Zeichen der Figur geben dieses Verhalten schematisch an.

Das Resultat der inneren Polarisation des Nerven besteht also darin, daß das Membranpotential an der Anode verstärkt, an der Kathode geschwächt wird. Die über die Achse der Faser konstruierte Kurve gibt die Stärke und Ausbreitung dieser Veränderung wieder. Alle einzelnen Erscheinungen dieses Vorganges verhalten sich so, wie sie am Nerven und Kernleiter beschrieben sind. Die Polarisation an der Kathode kann sich bei starkem Strom schließlich auch umkehren.

Die elektrotonischen Ströme des Nerven verhalten sich nun bei der Reizung des Nerven ganz ebenso, wie die Ströme des ruhenden Nerven. Sie erleiden dabei eine negative Schwankung (Bernstein). Am deutlichsten läßt sich dies beobachten, wenn

Fig. 47.

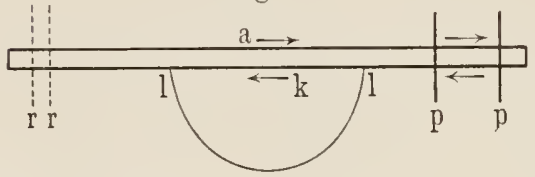

Negative Schwankung der elektrotonischen Ströme. man (Fig. 47) zwei symmetrische Längsschnittpunkte 77 ableitet, auf der einen Seite den Nerven in $p p$ polarisiert und ihn auf der anderen Seite in $r r$ reizt. Im unpolarisierten Zustande bewirkt die Reizung bei geschlossenem Kreise von $7 l$ keine Ablenkung, da sich die Reizwelle ohne Dekrement fortpflanzt und die entgegengesetzten Phasen sich daher aufheben. Polarisiert man aber den Nerven in $p p$, so entstehen elektrotonische Ströme zwischen 77 in der Richtung $a$ oder $l$, und bei der Reizung eine negative Schwankung derselben. Dies erklärt sich nun nach der Membrantheorie in folgender Weise. Bei der Reizung wird die Membran permeabler, und es sinkt das Membranpotential durch Vereinigung der Ionen. Im Bereich der Anode, des höheren Membranpotentials, ist die negative Schwankung daher stärker, im Bereich der Kathode dagegen schwächer. Wenn sich die Reizwelle von $p$ nach 77 fortpflanzt, so wächst sie, wenn sie sich der Anode nähert, und sie nimmt ab, wenn sie sich der Kathode nähert. Es muß aber hinzugefügt werden, daß bei diesem Ver'such die Reizung eine starke sein inuf, damit, wie wir bald zehen werden, die Änderungen der Reizbarkeit an der Anode und Kathode keinen Einfluß auf das Resultat ausüben. Versuche, welche mit dem Rheotom ausgeführt 
wurden (L. Hermann), sprechen für unsere Deutung. Untersuchungen hierüber mit dem Kapillarelektrometer lassen sich ebenso deuten (Gotch und Burch). Weitere Versuche in dieser Richtung sind wünschenswert.

Man hat den Zustand, welcher bei Durchströmung der Nerven und Muskeln an der Anode entsteht, mit Anelektrotonus und den an der Kathode mit Katelektrotou us bezeichnet. Schon von Pflüger ist das Entstehel und Verschwinden dieser Zustände als Ursache der elektrischen Reizung bei Schließung und Öffnung des Stromes angesehen worden. Er stellte auf Grund seiner Untersuchungen das allgemein bestätigte Gesetz der polaren Erregung auf. Dasselbe lautet: „Beim Schließen des Stromes findet die Erregung an der Kathodestatt, beim Öffnen desselben an der Anode." Dieses Gesetz läbt sich nun aus der Membrantheorie deuten. Gehen wir davon aus, daß bei der Erregung eine Zustandsänderung der Plasmamembran eintritt, infolge deren sie permeabler wird und die Ionen der Doppelschicht sich miteinander vereinigen, so tritt ein solcher Prozeß beim Schließen des polarisierenden Stromes an der Kathode ein, nicht aber an der Anode. An der Kathode vereinigen sich die Ionen der Doppelschicht mit denen der Elektrolyse, und es erfolgt dadurch eine Zustandsänderung der Plasmamembran, welch $\theta$ mit Erregung identisch ist. Das sehr labile Gleichgewicht der Membranteilchen wird durch die Verminderung der Doppelschicht gestört, wie dies durch Reizung anderer Art geschieht. An der Anode dagegen erhöht sich die Kíraft der Doppelschicht, und dies hat die entgegengesetzte Wirkung. Das Gleichgewicht der Membranteilchen wird ein stabileres, also tritt keine Erregung ein. Man kann auch nach der Höberschen Hypothese (s. oben S. 101) sagen, an der Kathode finde eine Lockerung, an der Anode eine Verdichtung der Membranteilchen statt. Doch müssen wir uns damit einen chemischen Prozeß verbunden denken, welcher bei der Erregung in Spaltung und Oxydation besteht. Diesen eigentlichen chemischen Erregungsprozeß müssen wir von dem elektrochemischen der Ionen wohl unterscheiden, obwohl sie beide ursächlich und zeitlich sich gegenseitig bedingen; denn daran müssen wir festhaiten, daß die elektrischen Prozesse nicht der krregungsvorgang selbst sind, sondern nur das Zeichen für denselben. 
Bei der Öffnung des Stromes findet nun eine innere Depolarisation statt. Das an der Anode gestiegene Membranpotential sinkt, und das an der Kathode verminderte steigt. An der Anode findet Vereinigung von Ionen der Doppelschicht statt, und dieser Vorgang erschüttert die Membranteilchen, an denen die Ionen haften. An der Anode tritt daher Erregung ein. An der Kathode hingegen werden die Membranteilchen, indem sich die Doppelschicht vermehrt, wieder in den stabileren Zustand versetzt, also kann hier keine Erregung stattfinden.

Nicht nur das Gesetz der polaren Erregung, sondern auch die Veränderungen der Reizbarkeit im An- und Katelektrotonus lassen sich aus der Membrantheorie herleiten. Nach den

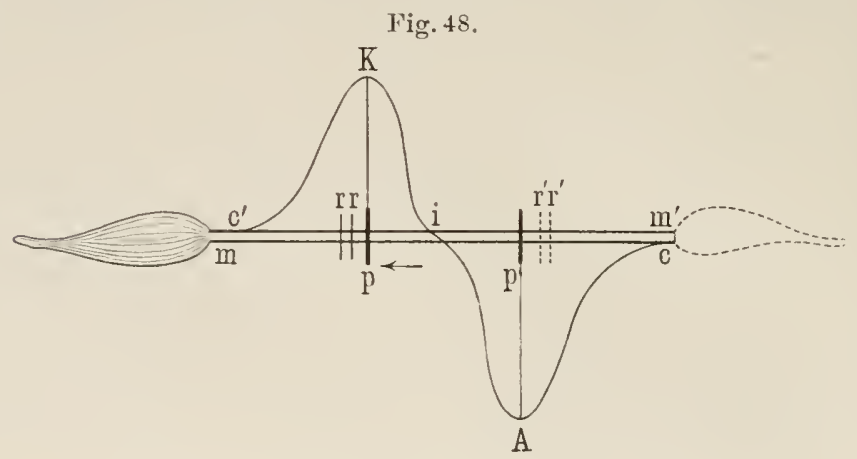

Änderungen der Reizbarkeit im An- und Katelektrotonus.

Forschungen von P flüger tritt im Bereich der Anode eine Herabsetzung, im Bereich der Kathode eine Erhöhung der Reizbarkeit ein. Dies folgt schon unmittelbar aus den obigen Darlegungen. Im Bereich der Anode sind die Membranteilchen durch Erhöhung des Membranpotentials in einen stabileren Zustand versetzt, und da eine jede Reizung in einer Auslösung potentieller Energie besteht, so bedarf es nun eines stärkeren Reizes daselbst, um Erregung hervorzubringen; die Reizbarkeit ist vermindert, die Reizschwelle ist erböht. Das Umgekehrte ist während der Polarisation an der Kathode der Fall. Die Membranteilchen sind in einen labileren Zustand versetzt; die Reizbarkeit muß also erhöht, die Reizschwelle muß vermindert sein. Diese Erscheinungen beobachtet man bekanntlich am besten an einem Nervmuskelpräparat 
(s. Fig. 48), indem man durch eine Nervenstrecke $p p$ einen konstanten Strom leitet. Ist der Strom zentrifugal (absteigend) nach dem IIuskel $m$ zu gerichtet, so gibt die Kurve $c A K c^{\prime}$ die Änderung der Reizbarkeit an. Die erböhte Reizbarkeit im Katelektrotollus geht in der intrapolaren Strecke $p p$ durch einen Indifferenzpunkt $i$ in die verminderte Reizbarkeit des Anelektrotonus über. Beide Zustände dehnell sich in den extrapolaren Strecken so weit ans, als die Veränderung des Membranpotentials reicht.

Man kaun die Reizbarkeit in $r r$ mit elektrischen Reiz, Induktionsstrom oder Schließen und Öffnen eines konstanten Stromes, oder auch mit chemischem Reiz durch einen Tropfen konzentrierter ClNa-Lösung prüfen und erhält dasselbe Resultat.

Denken wir uns den Muskel $m^{\prime}$ auf der anderen Seite, so ist der polarisierende Strom in $p p$ zentripetal (aufsteigend) gerichtet, und in $r^{\prime} r^{\prime}$ konstatiert man herabgesetzte Reizbarkeit und höhere Reizschwelle bei Anwendung irgend welchen Reizes.

Für gewöhnlich ist während der Stromdauer an Kaltblüternerven keine Erregung zu konstatieren. Dagegen sieht man am kuraresierten Muskel, da@ an der Kathode eine beständige schwache Kontraktion vorhanden ist, die sich an die Schließungszuckung anschließt. Überhaupt lälst sich an einem langen kuraresierten Muskel (Musc. sartorius) das Gesetz der polaren Erregung direkt beobachten. Bei der Schließung des Stromes zuckt nur die Kathodenhälfte des in der Mitte fixierten Muskels, bei der Öffnung nur die Anodenhälfte. Die Reizleitung zur anderen Hälfte wird durch Herabsetzung der Reizbarkeit gehemmt. Nicht selten tritt anch bei Polarisation eines Nerven Dauererregung auf, wenn derselbe sehr reizbar ist, häufig auch bei Warmblüternerven. Dieser Zustand erklärt sich sehr wohl daraus, daß auch während der Stromdauer im Bereich der Kathode die Vereinigung von Ionen nicht ganz aufhört. Da die Membran permeabler geworden ist, so geben Ionen der Elektrolyte schneller hindurch als in unpolarisiertem Zustande und begegnen denen der Elektrolyse. Der im Moment der Schließung in grölserer Intensität einsetzende Vorgang der Vereinigung von Ionen in der Doppelschicht setzt sich also wäbrend des Stromes in schwächerem Maße fort und kann in einer Dauererregung zum Vorschein kommen. Nicht so im Bereich der Anode. Hier häufen sich vielmehr die Ionen in der Doppelschicht der Membran an, ohne sich zu vereinigen. Die 
Nembran wird undurchlässiger als im unpolarisierten Zustand. Während der Stromdauer kann hier eine Erregung nicht stattfinden. Dagegen sieht man nicht selten, daß beim Öffnen des Stromes die Zuckung. in einen Tetanus übergeht, den Ritterschen Öffnungstetanus. Derselbe erklärt sich aus der längeren Dauer der hierbei erfolgenden Depolarisation, nachdem sich eine gröbere Menge von lonen angehäuft hat.

Auch die Umkehr der Erregbarkeiten, welche Pflüger nach dem Anelektrotonus und Katelektrotonus festgestellt hat, läßt sich als Folge der Theorie deuten. Unmittelbar nach der Öffnung des polarisierenden Stromes beobachtet man im Bereich der Anode eine erhöhte Reizbarkeit, im Ber'eich der Kathode eine verminderte Reizbarkeit, die bald in die gewöhnliche übergeht An der Anode versetzt die Vereinigung der Ionen die Membranteilchen in einen labileren Zustand, an der Kathode werden dieselben durch Ansammlung der lonen in einen stabileren Zustand versetzt. Während der Stromdauer haben sich in den Membranteilchen an der Anode chemische Energien angehäuft, welche nach der (̈)ffuung leicht auslösbar sind, während an der Kathode die Membranteilchen beständig chemische Energien auch während der Stromdauer ausgegeben haben, so daß der Rest derselben schwerer auslösbar geworden ist.

Es stimmen diese Erklärungen mit der allgemeiueren von l'flüger gegebenen Vorstellung wohl überein, nach welcher er in den Molekülen der reizbaren Substanz zwei einander entgegengesetzte Kräfte voraussetzt, eine Molekularspannung und eine Molekularhemmung, und im Anelektrotonus eine Verstärkung, im Katelektrotonus eine Schwächung der Hemmung annimmt. Während der Stromdauer häufen sich daher Molekularspannungen durch den Stoffwechsel an der Anode an, an der Kathode sinken sie auf ein geringeres MaB herab. Daher beim Schließen die Erregung an der Kathode, beim Öffnen an der Anode, und daher auch die Unkehruug der Reizbarkeiten nach der Öffnung des Stromes; denn es überwiegt alsdann eine gewisse Zeit au der Anode die Molekularspannung, an der Kathode die Molekularhemmung bis zur Wiederherstellung des normalen Gleichgewichtes.

In Übereinstimmung mit diesen Vorstellungen hat E. Hering den Satz aufgestellt: Im Katelektrotonus herrscht die Dissimilierung der lebenden Substanz vor, im Anelektrotonus die Assimi- 
lieruug. Nach der Öffnung des Stromes tritt der umgekehrte Zustand auf, wie dies auch allgemeim beim Wechsel von Erregung und Ruhe der Fall ist. Wenn diese Vorstellungen richtig sind, dann müßte am Muskel nachzuweisen sein, daß an der Kathode eine Wärmeproduktion stattfindet, an der Anode keine oder nur eine geringe, elıer sogar eine Wärmeabsorption. Versuche dieser Art, unter Berücksichtigung der Jouleschen Stromwärme, sind noch nicht ausgeführt.

Von diesen Vorstellungen ausgehend, hat man sich zu denken, $\mathrm{da} B$ in einer reizbaren Substanz die hervorgebrachte Leistung $L$ cet. par. im wesentlıchen volı drei Variablen abhängig sein muß, erstens von der in ihr vorhandenen potentiellen Energie $P$, welche durch den Reiz auslösbar ist, zweitens von der Größe des Reizes $r$ und drittens von dem Grade der Auslösbarkeit, was wir Reizbarkeit nemnen, und diese wollen wir durch den reziproken Wert der Reizschwelle $b$ ausdrücken. Es ist dann also die Leistung $I$. eine gewisse Funktion von $P$ und $r$ und $b$, welche Null werden muß, wenn $P$ oder $r-b$ gleich Null wird. Wir können daher dieser Funktion die allgemeine Form geben:

$$
L=(r-b) \cdot P \cdot f[(r-b), P] .
$$

Das einfachste mechanische Beispiel hierfür ist ein Wasserreservoir, welches unten durch einen Hahn verschlossen ist. Wird derselbe geöffnet, so strömt Wasser aus, d. h. potentielle Energie der Wassersäule wird in kinetische umgesetzt. Der Hahn kaun leicht oder schwer drehbar, also die auslösende Kraft bei derselben Leistung eine kleinere oder größere sein. Die Leistung wird aber Null seiu, wenu die Druckhöhe Null geworden oder der Hahn gar nicht mehr drehbar ist. Ein Beispiel für chemische Energie bieten die explosiven Substanzen, deren potentielle Energie durch geringe auslösende Kräfte in kinetische umgesetzt werden kann. Durch gewisse Einwirkungen kann ihre Empfindlichkeit, d. h. Reizbarkeit, erhöht, durch andere herabgesetzt werden.

Von diesen Gesichtspunkten ausgehend, müssen wir noch eimual auf die negative Schwankung des polarisierten Nerven in Beziehung zur Reizbarkeit im An- und Katelektrotonus zurückkommen. Die verminderte Reizbarkeit im Anelektrotonus scheint dem erhöhten Membranpotential und der verstärkten Reizwelle in diesem Zustande zu widersprechen, ebenso die erhöhte Reizbarkeit 
im Katelektrotonus dem verminderten Membranpotential und der geschwächten Reizwelle. Dem ist aber nicht so. Auch für die negative Schwankung ist im Anelektrotonus die Reizbarkeit eine geringere (Reizschwelle eine höhere) und im Katelektrotonus die Reizbarkeit eine höhere (Reizschwelle eine geringere). W'enu man aber im Anelektrotonus die Reizstärke vermehrt, so kann man nicht nur am Muskel des Nerven eine ungeschwächte, sonder'n unter günstigen Umständen sogar eine verstärkte Zuckung erhalten (Bernstein), entsprechend der verstärkten Reizwelle. Wenn man im Katelektrotonus die Reizstärke vermehrt, so findet man, namentlich nach längerer Polarisation, eine geschwächte Maxinalzuckung am Muskel vor, entsprechend der verminderten Reizwelle. Um dies zu beobachten, muß man, wie in Fig. 47, die Reizelektroden $r r$ und $r^{\prime} r^{\prime}$ nahe an die polarisierenden $p p$ heranbringen und mit stärkeren Strömen reizen. Denkt man sich bei dieser Anordnung an Stelle der Muskeln den Nerven an den Enden zum Galvanometer abgeleitet, so erhält man beim Aufsuchen der Reizschwelle für die negative Schwankung im Katelektrotonus erhöhte, im Auelektrotonus verminderte Reizbarkeit. Bei Verstärkung der Reizströme in $r r$ jedoch erscheint im Anelektrotonus die verstärkte, im Kíatelektrotonus die geschwächte negative Schwankung (Reizwelle). Es geht dies aus der Formel (13) klar hervor. Im Anelektrotonus wird $b$ und $P$ vermehrt, statt $b$ setzen wir $b_{a}$ und $P_{a}$ statt $I$. Also erhalten wir.

$$
L_{a}=\left(r-b_{a}\right) \cdot P_{a} \cdot f\left[\left(r-b_{a}\right), P_{a}\right] \text {. }
$$

Die Leistung ist Null, wenn $r=b_{a}$ wird, also wird bei schwachen Reizen an der Grenze der Reizschwelle $b_{a}$ und zwischen $b$ und $b_{a}$ die Leistung $L_{a}$ kleiner als $L$. Werden aber die Reize so stark, daß $b_{a}$ gegen $r$ verschwindet, so überwiegt der Einfluß von $P_{u}>P$, und $L_{a}$ wird größer als $L$. Im Katelektrotonus setzen wir als Reizschwelle $b_{k}<b$ und als Energie $P_{k}<P$ und erhalten:

$$
L_{k}=\left(r-b_{k}\right) \cdot P_{k} f\left[\left(r-b_{k}\right), P_{k}\right] \text {. }
$$

Bei schwachen Reizen ist $L_{k}>L$, weil $\left(r-b_{k}\right)>(r-b)$ ist, bei stärkerem Reize, wenn $b_{k}$ und $b$ gegen $r$ verschwinden, wird $L_{k}<L$, weil $P_{k}<P$ ist.

Das Gesetz der elektrischen Reizung ist oft zum Gegenstand der Untersuchung und Erörterung gemacht worden. Zuerst hat du Bois-Reymond ein solches Gesetz aufgestellt, 
welches lautet: "Nicht der absolute Wert der Stromdichtigkeit in jedem Augenblick ist es, auf den der Bewegungsmerv mit Zuckung des zugehörigen Muskels antwortet, sondern die Veränderung dieses Wertes von einem Augenblick zum anderen, und zwar ist die Anregung zur Bewegung, die diesen Veränderungen folgt, um so bedeutender, je schneller sie bei gleicher Gröbe vor sich gingen, oder je größer sie in der Zeiteinheit waren."

Dem $d u$ Boisschen Gesetz liegt die Beobachtung zugrunde, daß im allgemeinen ein konstanter Strom während seiner Dauer nicht reizt, und daß Erregungen nicht nur beim Schließen und Öffnen, sondern auch bei schnellen Schwankungen der Stromstärke bzw. der Stromdichte (Stromdichte $\left.=\frac{\text { Stromstärke }}{\text { Querschnitt }}\right)$ in positiver oder negativer Richtung auftreten.

Je schneller man die Stromstärke (setzen wir den Querschnitt als konstant bleibend voraus) sich ändern läßt, desto stärker ist die Reizung. Dies kann man mit einem Schwankungsrheochord beobachten, bei welchem man den Schleiflontakt (s. Fig. 5, S. 7) sich schnell bewegen läßt.

Das du Boissche Gesetz ist aber nur in gewisser Annäherung richtig. Es ist richtig, daß die Schwankung der Stromstärke die einflubreichste Variable ist; aber schon Pfl üger hat eingewendet, daß auch die konstant bleibende Stromstärke nicht nur die Erregbarkeit verändert, sondern auch an der Kathode Erregungen auslösen kann. Also die absolute Stromstärke muß auch einen Einfluß auf die Größe der Erregung haben. A. Fick hat ferner gezeigt, daß auch die Dauer des Stromes einen Einfluß besitzt. Er zeigte, daß bei kurz dauerudem konstanten Strom die Erregung mit zunehmender Dauer bis zu einer gewissen Grenze wächst.

Es kann also die Größe der Erregung nicht bloß eine Funktion der Stromschwankung sein, welche wir durch den Differentialquotienten der Stromstärke nach der Zeit $\frac{d i}{d t}$ ausdrücken, sondern es müssen auch die absoluten Werte von $i$ und von $t$ als Variable angenommen werden. Allgemein ansgedrückt würde dieses Gesetz lanten, wenn $\eta$ die Erregung zur Zeit $t$ ist:

$$
\frac{d \eta}{d t}=F\left(\frac{d i}{d t}, i, t\right)
$$


Es ist $d \eta$ der Zuwachs der Erregung zur Zeit $t$ in der Zeit $d t$. Also würde die Gesamterregung von Zeit 0 bis $t_{1}$ sein:

$$
\eta=\int_{0}^{t_{1}} F\left(\frac{d i}{d t}, i, t\right) d t .
$$

Wir wollen zunächst zeigen, daß der Prozeß der elektrischen Reizung nach der Membrantheorie mit dieser Formel in Übereinstimmung steht. Die innere Polarisation ist ja nach dieser Theorie auch die Ursache der elektrischen Reizung. So lange die Stromstärke $i$ schwankt, ändert sich das Membranpotential an der Anode und Kathode, so lange muß bei Zunahme von $i$ an der Kiathode, bei Abnahme von $i$ an der Anode Erregung stattfinden. Aber auch wenn $i$ lionstant geworden und $\frac{d i}{d t}$ Null ist, bleibt an der Kathode, wie oben gezeigt, eine Danererregung durch schwächere Ionenvereinigung bestehen. Und encllich steigt die innere Polarisation nicht momentan zum Maximum, sondern erst innerhalb einer gewissen Zeit, strenge genommen, erst nach unendlicher Zeit, wie bei jeder physikalischen Polarisation. Es geht hieraus deutlich hervor, daß die Erregung in erster Linie von den Stromschwankungen abhängig sein muß, in viel geringerem Maße von der absoluten Stromstärke und von der Dauer des Stromes. Man hat daher nach $\mathrm{du}$ Bois geglaubt, die beiden letzteren Variablen vernachlässigen zu können und die Erregung nur als Funktion von der Stromschwankung $\frac{d i}{d t}$ angesehen. Diese Annahme kann aber nicht zu ganz richtigen Resultaten fübren.

Es muß bei weiterer Behandlung dieses Gegenstandes zunächst die Grundfrage erörtert werden, welche bei Versuchen zu beobachtende Größe wir als Zuwachs- oder Elementarerregung $d \eta$ und welche wir demnach als Gesamterregung $\eta$ in dem Zeitmoment $t$ zu messen haben. Diese Frage ist bisher nicht befriedigend behandelt worden. In allen Versuchen dieser Art ist bisher nur die Muskelzuckung als Reagens der Nerven-oder Muskelreizung benutzt worden. Aber es ist klar, daß diese kein proportionales Maß für die Muskelerregung, noch weniger für die Nervenerregung ist. Tom Muskel wissen wir, daß die gesamte 
in ihm durch den Reiz ausgelöste Energie nur zum Teil als Arbeit erscheint, zum anderen Teil als Würme. Als Maß für die gesamte Erregung $\eta$ rom Moment Null bis zur Zeit $t$ kann aber nur die gesamte in ihm freigewordene Energie angesehen werden, die wir durch Arbeits- und Wärmemessung finden. Die Elementarerregung $d \eta$ ist hiernach der Zuwachs an Energie, welche in jedem Zeitteilchen $d t$ ausgelöst wird. Versuche dieser Art fehlen bis jetzt gänzlich und ließen sich nur am Muskel ausfübren. Wenn man also Ströme verschiedener Stärke, Scbnelligkeit der Schwankung, und Zeitdauer auf Nerv und Muskel hat einwirken lassen und die schließliche Höhe der Muskelzuckung als Maß der Erregung betrachtet hat, so kann dieses Maß nicht als richtig angesehen werden.

Messende Versuche, welche über diesen Gegenstand bisher angestellt sind, beschränken sich darauf, den elektrischen Minimalreiz zu finden, welcher eben eine Erregung auslöst, die sogenannte Reizschwelle, bei verschiedener Art der Reizung. Hoorweg ${ }^{1}$ ) hat sich der Kondensatorentladung bedient, deren Verlauf man genau kennt, und untersucht, wie groß das Potential bei verschiedener Kapazität des Kondensators sein muß, um die Reizschwelle zu erreichen. Wenn $P$ das Potential, $R$ den Widerstand des Kreises und $C$ die Kapazität bedeutet, so findet or die Formel:

$$
P=a \cdot R+\frac{b}{C}
$$

a und $b$ sind zwei Konstanten, die aus zwei Beobachtungen gefunden werden. Die Kapazität eines Kondensators, z. B. einer Leidener Flasche usw., ist bekanntlich diejenige Elektrizitätsmenge, welche sich beim Potential Eins auf den Belegungen befindet, und zwar wächst sie proportional der Oberfläche der Belegungen und umgekehrt proportional dem Abstand der Belegungen voneinander. Ist also $Q$ die Elektrizitätsmenge, so ist $C=\frac{Q}{P}$. Die Formel von Hoorweg gibt also an, daß die Potentialspannung $P$ für die Reizschwelle um so kleiner wird, je größer die Kapazität ist, bei gleichbleibendem Widerstand $R$, daß sie dagegen bei gleich-

1) Siehe Pflügers Archiv 1892-1901, bes. Bd. 52, 71, 87. 
bleibender Kapazität $C$ mit dem Widerstand $R$ wächst, aber in beiden Fällen nicht proportional. Da $P=\frac{Q}{C}$ ist, so erhält man auch die Beziehung:

$$
\frac{Q}{C}=a r+\frac{b}{C}, \text { oder: } Q=a \cdot C \cdot R+b,
$$

d. h. bei gleichbleibenden Widerstand des Líreises nimmt die zur Minimalreizung notwendige Elektrizitätsmenge $Q$ mit zunebmender Kapazität zu, aber auch nicht proportional, da eine zu addierende Konstante $b$ noch hinzutritt.

Eine ähnliche einfache Formel für die elektrische Reizschwelle bei Reizung mit kurz dauernden konstanten Strömen ist auch von Georg Weiss ${ }^{1}$ ) anfgestellt worden. Diese Ströme wurden mit einem Schußapparat hergestellt, durch den die Leitung geschlossen und schnell unterbrochen werden konnte. Er gelangt zu der Beziehung: $Q=a+b t$, worin $a$ und $b$ zwei Konstanten sind. Da in diesem Falle $Q$, die Elektrizitätsmenge, gleich Intensität $i$ mal Zeit ist, so ist auch:

$$
i \cdot t=a+b t \text { oder } i=\frac{a}{t}+b
$$

Das heißt also, die Intensität der Reizschwelle muß um so größer sein, je kleiner die Dauer $t$ des Stromes ist, oder sie wird kleiner mit zunehmender Dauer. Bleibt der Strom geschlossen, ist $t=\infty$, so wird $i=b$. Für kleine Zeiten gibt auch diese Formel annäbernd mit der Beobachtung stimmende Resultate, wie die von Hoorweg. Es stimmt die Formel von Weiss auch gut zu den älteren Versuchen von A. Fick, die oben (S. 141) erwähnt sind.

Diese Untersuchungen gingen nicht von den inneren Prozessen aus, welche der Strom in den Organen direkt hervorruft, wie wir es in den obigen Betrachtungen voln Standpunkt der Membrantheorie getan haben. Dagegen ist von Nernst ${ }^{2}$ ) auf Grund von Versuchen mit sehr frequenten Wechselströmen von Wechselstrommaschinen eine Theorie der elektrischen Reizung anfgestellt worden, welche von der Betrachtung der Elektrolyse in den Organen ausgeht. Er gelangt hieraus für Wechselströme

1) Archiv. italien. d. biologie 35 .

2) Sitzungsber. d. Berl. Akad. 26, 524 (1908): Pflügers Arch. 1:2, 293 (1908). 
von der Schwingungszahl $m$ und der Amplitude der Intensität $i$ zu dem einfachen Gesetz, daß bei der Reizschwelle die Beziehung besteht:

$$
\frac{i}{\sqrt{m}}=\text { Konst . . . . . . . . }
$$

Diese Versuche sind an Empfindungsnerven der Haut des Menschen und auch an motorischen Froschnerven angestellt worden. Folgende Tabelle zeigt ein solches Experiment. (Pflügers Arch. 1:2:3, 293, 1908.)

\begin{tabular}{r|c|c|c|c}
\hline$m$ & $i$ beob. & $i$ ber. & $\begin{array}{c}\text { Differenz } \\
\text { Proz. }\end{array}$ & $i / \sqrt{m} \cdot 10^{3}$ \\
\hline 105 & 0,81 & 0,78 & $-4,2$ & 78 \\
136 & 0,88 & 0,92 & $+4,6$ & 75 \\
785 & 2,16 & 2,21 & $+2,3$ & 77 \\
960 & 2,41 & 2,47 & $+2,9$ & 77 \\
2230 & 3,85 & 3,73 & $-3,1$ & 81
\end{tabular}

Mittel: $i=0,079 \mathrm{~J} \mathrm{~m}$.

Nernst geht von der Ansicht aus, daß die Membranen der Nerven und Muskeln nicht, wie Ostwald annahm, Ionensiebe darstellen, sondern daß sie gegenüber der Gewebsflüssigkeit ein zweites Lösungsmittel bilden und sich also so verhalten, wie es Nernst und Riesenfeld (s. oben S. 37) an zwei miteinander nicht mischbaren Lösungsmitteln, in denen sich ein Elektrolyt verteilt, beobachtet hatten, wenn durch die Berührungsfläche ein elektrischer Strom geleitet wird. In diesem Falle tritt nicht wie an der Ostwaldschen semipermeablen Membran eine Abscheidung von Ionen ein, sondern nur eine Konzentrationsänderung des Elektrolyten. Diese Konzentrationsänderung sieht Nernst als die Ursache der Erregung an und setzt dieselbe der Größe der Erregung proportional. Der Konzentrationsänderung durch Elektrolyse wirkt aber die Diffusion entgegen, und aus einer aus diesen Voraussetzungen aufgestellten Differentialgleichung für die Änderung der Konzentration an einer Berührungsfläche in jedem kleinsten Zeitteilchen und der Annahme eines Schwellenwertes der Konzentrationsänderung für die Reizschwelle entwickelt er obige Formel für den Schwellenwert von $i$ bei verschiedener Schwingungszahl $m$ der Wechselströme. 
Die Nernstsche Theorie reicht indessen nicht aus, um zu einer allgemeinen Lösung des Problems der elektrischen Reizung zu führen, wie es durch die obige Formel (15) (S. 142) aufgestellt worden ist. Sie gibt keine Erklärung für das Gesetz der polaren Erregung, daß die Erregung beim Schließen an der Kathode und beim Öffnen an der Anode eintritt, und das liegt daran, daß sie keine bestimmte Voraussetzungen über den Ort der inneren Elektroden an den Nerven- und Muskelfasern macht, welche der Struktur derselben entsprechen. Sie nimmt nur eine Anode an der einen Grenze der beiden Lösungsmittel und eine Kathode an der anderen Grenze an und identifiziert somit die Nerven- und Muskelfasern schematisch mit einem U-Rohr, dessen Mitte etwa mit Phenol und dessen beide Euden mit Wasser gefüllt sind. Nach dieser Vorstellung hätte man die Außenflüssigkeit als das eine (Phase 1) und den ganzen Inhalt der Fasern als das andere Lösungsmittel (Phase 2) anzusehen. Diese genügt aber keineswegs, um die Erscheinungen der Eigenströme zu erklären und den Zusammenhang derselben mit der elektrischen Reizbarkeit zu deuten. Vielmehr gingen wir von der wohlbegründeten Vorstellung einer semipermeabeln Plasmamembran an der Oberfläche der Fasern aus, deren beide Oberflächen sowohl im Bereiche der äußeren Anode wie im Bereiche der äußeren Kathode Elektroden bilden, und die schon im unpolarisierten Zustande vermöge der Osmose eine elektrische Doppelschicht besitzt. Wollte man die Nernstsche Theorie auf diese Struktur der Fasern und Zellen anwenden, so würden die Konzentrationsänderungen, wenn man die Membran nicht als Diaphragma, sondern als Lösungsmittel (Phase) behandelt, an beiden Oberflächen entgegengesetzt und gleich ausfallen und sich demnach in ihrer Wirkung auf das Plasma aufheben ${ }^{1}$ ).

Indessen kann man die Nernstschen Formeln recht gut auf die Membrantheorie übertragen. Man braucht nur statt Konzentrationsänderung des Elektrolyten Änderung des .Membranpotentials durch die innere Polarisation zu setzen und gelangt

$\left.{ }^{1}\right)$ In ähnlicher Weise zeigt auch Cremer (siehe Handbuch der Physiologie von Nagel, Bd.IV, S. 875), daß die beiden Phasengrenzkräfte an diesen Oberflächen sich aufheben und zur Erklärung des Eigenstromes nur die osmotische Potentialdifferenz übrig bleibt. 
dann zu ganz demselben Ergebnis $\left.{ }^{1}\right)$. Aus diesen Gründen haben wir in der ganzen theoretischen Darstellung an der Ostwaldschen Hypothese festgehalten, nach welcher die organischen Membranen Diaphragmen für die Moleküle und Ionen der Elektrolyte bilden, gegenüber der Nernstschen Hypothese, nach welcher sie Lösungsmittel (Phasen) sind. Wie sich die Sache in Wirklichkeit verhält, soll damit nicht entschieden werden; denn es besteht auch die Möglichkeit, daß sich die lebenden organischen Membranen teils als Diaphragmen, teils als Lösungsmittel verhalten. Die Overtonsche Lipoidmembran würde nur als Lösungsmittel anzusehen sein, doch auf diese konnten wir, wie oben gezeigt, die elektrische Membrantheorie nicht beziehen. Die Plasmamembran hingegen ist von uns als reines Diaphragma behandelt worden. Doch ist es hiernach nicht ausgeschlossen, daß ilnre Teilchen zugleich Elektrolyten und Nichtelektrolyten gegenüber als Lösungsmittel zu betrachten sind, wie dies wahrscheinlich bei jeder Kolloidsubstanz der Fall ist.

Wie schon oben bemerkt, beziehen sich alle bisherigen Untersuchungen dieser Art nur auf die Ermittelung der Reizschwelle, d. h. also auf den Fall, in welchem die Erregung $\eta=0$ wird. Dadurch kann natürlich eine Lösung der aufgestellten Funktion von $\eta$ in Formel (15) nicht herbeigeführt werden. Denken wir die Integration dieser Formel gelöst, und wir erhielten das Resultat: $\eta=\varphi(i, t)$, so stellt diese Gleichung bekanntlich eine Fläche im Raum in dem Koordinatensystem $\eta, i, t$ dar. Die angestellten Versuche geben uns aber nur den Fall, in welchem $\varphi(i, t)=0$ ist, d. h. in welchem die Erregung bei den Reizschwellen von $i$ und $t$ eben Null wird. Wir finden also von dieser gesuchten Fläche nur die Kurve, in welcher dieselbe die $i t$-Ebene schneidet. Um dies dentlich zu machen, sei in Fig. 49 das System der Koordinaten $t, \eta, i$ dargestellt, und in der $i t$-Ebene wollen wir die für kurzdauernde konstante Ströme annähernd gültige Formel von G. Weiss: " $i=\frac{a}{t}+b$ oder

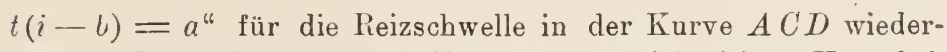
geben. Diese Kurve hat die Form einer gleichseitigen Hyperbel,

$\left.{ }^{1}\right)$ Eine weitere Ausführung dieses Gegenstandes will ich mir in einer speziellen Abhandlung vorbehalten. Der Autor. 
welche um das Stück $b$ auf der $i$-Ordinate nach oben verschoben ist. Die Konstante $b$ ist die Reizschwelle für $i$, wenn die l'auer $t$ sehr groß, $=\infty$ ist. Die Kurve fällt steil aus dem Unendlichen von $i$ ab und schließt sich asymptotisch dem Werte $b$ an. Für alle diese zusammengehörigen Werte von $i$ und $t$ ist also die Erregung $\eta$ gleich Null. Denken wir uns nun für einen endlichen Wert von $\eta$ einen Punkt $M$ in der Fläche $\eta=\varphi(i, t)$ mit den bestimmten Koordinaten $t_{1} \eta_{1} i_{1}$ und nehmen wir an, daß für die Stromkurve, mit welcher wir reizen, in dem Zeit-

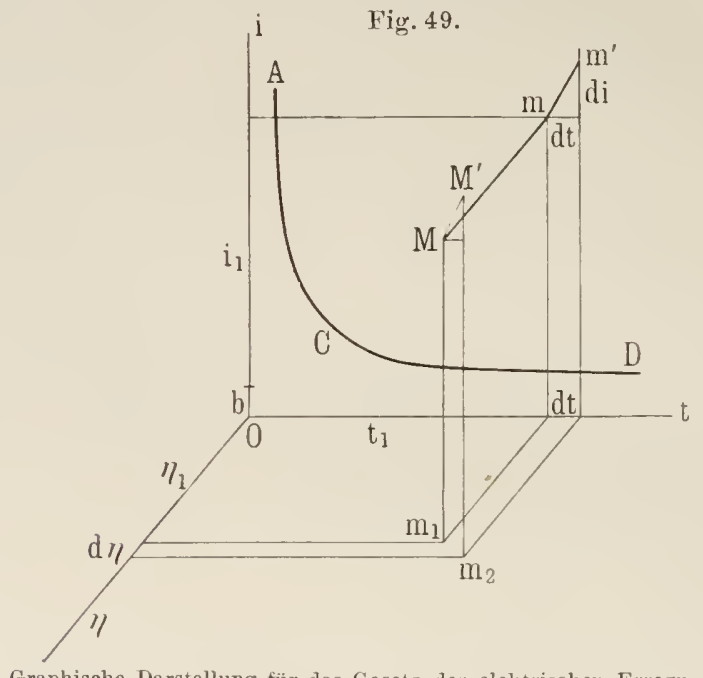

Graphische Darstellung für das Gesetz der elektrischen Erreguug.

punkte $t+d t$ der Punkt $\boldsymbol{l}^{\prime}$ dieser Fläche erreicht wird, so konstruieren wir auf und in der $\eta t$-Ebene die zu $M$ und $M I^{\prime}$ gehörigen Koordinaten, wie die Figur zeigt. Projizieren wir dann $M I M^{\prime}$ auf die $i t$-Ebene, so erhalten wir auf diese Weise den Wert $\frac{d i}{d t}$ in dieser Ebene, welcher zu dem Punkte $I I$ gehört. Wir erhalten ferner durch Projektion der Punkte $m_{1}$ und $m_{2}$ auf $\eta$ den zur Zeit $t$ hinzukommenden Zuwachs $d \eta$. Die Form der Kurve in der it-Ebene für die Reizschwelle ist nach den Formeln von G. Weiss, Hoorweg, Nernst und anderen eine verschiedene. Die Form der zugehörigen Fläche $\eta=\varphi(i, t)$ ist in allen Fällen 
noch ganz unbestimmt; wir wissen von ihr nur, daßsio die it- Ebene in der betreffenden Kurve schneidet. Eine ähnliche Konstruktion ließe sich für die Hoorwegsche und Nernstsche Formel ansführenl.

Bei der weiteren Ausführung der Formel (15) haben wir nach der Membrantheorie aber statt der Stromstärke $i$ (bzw. Stromdichte) das Membranpotential $P$ zu setzen, und haben daher noch die Abhängigkeit des $P$ von $i$ mit der Zeit zu berücksichtigen.

Es waren auch schon Untersuchungen über die Wirkung sehr frequenter unterbrochener Ströme und Wechselströme angestellt worden. Mit den gewöbnlichen Unterbrechern konnte man nur bis zu etwa 4000 bis 6000 Schwingungen in der Sekunde gelangen. Berustein ${ }^{1)}$ fand, daß bei der Reizschwelle der Ströme an dem Nerv-Muskelpräparat nur im Beginn der Reizung eine Zuckung anftritt, die .Anfangszuckung". Bei weiterer Verstärkung der Ströme gesellt sich Tetanus hinzu. Die Anfangszuckung erscheint, sobald die Frequenz der Ströme etwa die Zahl 300 in der Sekunde überschritten hat. Diese Erscheinung erklärt sich wohl am besten aus der Dauer der Reizwelle im Nuskel. Erst nach etwa ${ }^{1 / 300}$ Sekunde ist der Muskel nach einem Reiz nahezu in den anfänglichen Zustand znrückgekehrt und für denselben Reiz wieder gleich empfänglich (Refraktionsperiode nach Narey). Folgen sich die Reize schneller, so wirken die nachfolgenden schwächer oder schließlich gar nicht. Später hat man mit Hilfe der Tesla-Apparate sehr hochfrequente Wechselströme benutzt und gefunden, daß dieselben überhaupt nicht mehr, weder auf Nerv und Muskeln noch auf den lebenden menschlichen Körper erregend einwirken. Es hat dies wohl zum Teil einen rein physikalischen Grund, da die hochfrequenten Stromwellen mit zunehmender Schwingungszahl immer weniger in die Tiefe der Leiter eindringen und immer mehr an der Oberfläche derselben verlaufen. Bei Wechselströmen köunte dies aber uach der Nernstschen und der Membrantheorie noch darin seinen Grund haben, daß die Polarisatious - und Konzentrationsänderungen in den Organen nicht mehr schnell genug den Stromwechseln folgen können. Für gleichsinnige Stromwellen würde dies aber nicht

1) 1. c. Untersuchurgen 1871 . 
gelten, solldern sie müßten sich zur Wirkung eines konstanten Stromes addieren.

Schließlich haben wir noch den Einfluß der Winkelrichtung in Betracht zu ziehen, in welcher die elektrischen Ströme durch die Muskel- und Nervenfasern geleitet werden. Man hat gefunden, daß cet. par. das Maximum der Wirkung bei longitudinaler, das Minimum bei senkrechter Durchströmung eintritt, und daß die Wirkung dem Kosinus des Richtungswinkels annähernd proportional ist ${ }^{1}$ ). Am besten läßt sich diese Untersuchung an dem kuraresierten M. sartorius ausführen, der in einen rechteckigen, mit 0,6 Proz. ClNa-Lösung gefüllten Trog getaucht wird, durch welchen parallele Stromfäden geleitet werden. Ein $\nabla$ on zwei künstlichen Querschnitten begrenztes Mittelstückdieses Muskels, möglichst gerade gestreckt, reagiert in jeder beliebigen Winkelrichtung gegen die Stromfäden, selbst a uf starke Ströme, gar nicht.

Daraus habe ich schon früher gefolgert, daß die innere Polarisation, welche die Ursache der Erregung ist, nicht an der Oberfläche der Fasern stattfinden kann, sondern vielmehr an der Oberfläche der erregbaren Fibrillen oder noch feinerer Elemente derselben. Vom Standpunkte der Membrantheorie aus wird man daher die polarisierbare Membran den Fibrillen der Muskel- und Nervenfasern zuerteilen müssen. Die Unwirksamkeit parallelerStromfäden im obigen Versuche erklärt sich dann daraus, daß anodische und kathodische Polarisationen in jeder beliebigen Lage des Muskels an zwei gegenüber gelegenen Stellen der Fibrillen gleich stark sind und sich daher gegenseitig in ihrer Wirkung aufheben.

1) Leicher, Unters. a. d. physiol. Inst. zu Halle, Heft I, S. 1, 1888. Bernstein, ebenda S. 27; Naturw. Risch. 1888, III, S. 353. 


\section{Haut- und Drïsenströme. \\ Ihre Bedentung fïr die Sekretion und Resorption. Die Elektroosmose. Elektroosmotische Membrantheorie.}

Daß auch tierische Häute, auf demen eine Sekretion stattfindet, einen Strom erzeugen, wenn man sie von beiden Flächen ableitet, ist zuerst von E. du Bois-Reymond an der Froschhant beobachtet worden (1857). Man findet die äußere Fläche, also die sezernierende Oberfläche, negativ, die innere Fläche dagegen positiv, wenn man mit unpolarisierbaren Kochsalzelektroden untersucht. Die Kraft schwankt etwa zwischen 40 bis 90 Millivolt (Engelmann). Zerstört man die Haut durch Hitze, oder behandelt sie mit Chloroformdämpfen, so verschwindet der Strom. Man hat die gewöbnliche Richtung dieses Stromes von außen nach innen auch die "einsteigende" genannt (L. Hermann).

Solche Ströme beobachtet man auch an den sezernierenden Schleimhäuten des Magens und Darmkanals (Rosenthal), und sie besitzen auch hier eine einsteigende Richtung, d. h. sie sind von der sezernierenden freien äußeren Fläche nach der inneren hin gerichtet. Die sezernier ende Flächo ist also immer die ne gative. Diese. Ströme sind bei vielen Tieren, sowohl bei niederen Wirbeltieren, Amphibien und Fischen, wie bei höheren Warmblütern, an der äußeren Haut und den Schleimhảuten derselben festgestellt. In den meisten dieser Häute finden sich Drüsen verschiedener Form eingelagert und regelmäßig angeordnet, meist in Schlauch-, Röhrenoder Flaschenform dicht nebeneinander gestellt. Man hat daher die elektrische Kraft hauptsächlich diesen Drüsen und ihren Zellen zugeschrieben. Aber auch an Häuten, welche keine solche Drüsenformen enthalten, wie die Haut der Aale, zeigt sich ein solcher Strom (Hermann). Man muß daher den Sitz der elektrischen Kraft nicht nur in die eigentlichen Drüsenzellen, sondern auch in die Epithelzellen verlegen, welche diese Häute auf der äußeren Fläche bedecken. Dies ist auch histogenetisch insofern berechtigt, als ja bei der Entwickelung die Drüsenzelien aus den Epithelzellen 
nach Einstülpung der äußeren Hautfläche hervorgehen. Auch die Epithelzellen zeigen mehr oder weniger die Funktion der Sekretion; sie scheiden besonders Schleim ab oder erleiden gewisse chemische Veränderungen, wie die Verhornung in der Epidermis der höheren Wirbeltiere. In Fig. 50 a sieht man eine solche Haut schematisch dargestellt, besetzt mit sezernierenden Epithelzellen; in Fig. 50 b ist die Einstülpung derselben zur Bildung verschiedener Dräsenformen verdeutlicht, welche verschiedene formen, einfache Röhrchen und Bläschen und durch Teilung der Gänge zusammengesetzte röhren- und bläschenförmige Drüsen von größerem Umfang, wie die Speicheldrüsen, Pankreas usw. entstehen läßt.

Fig. 50 a u. b.

a.
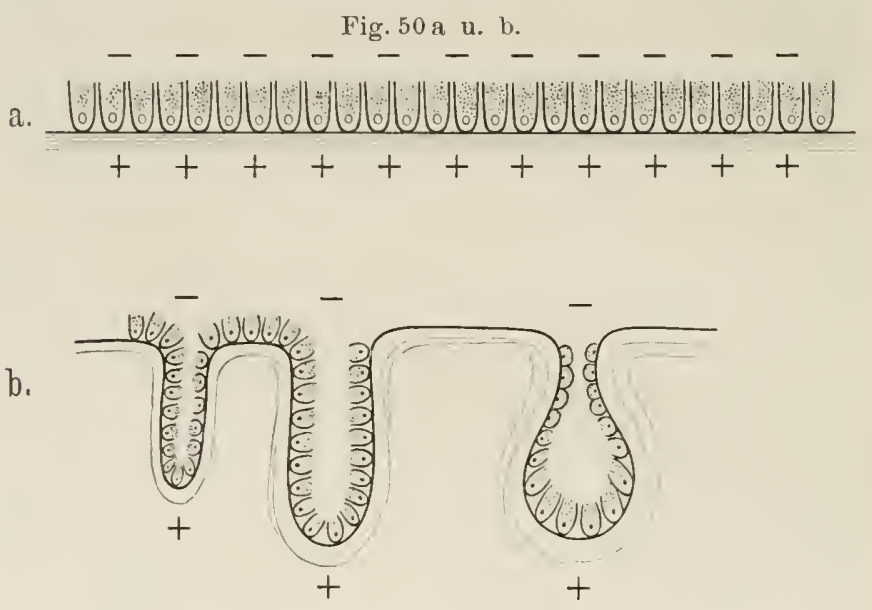

i) Haut mit Sekretionszellen (Schema), b) Einfache Drüsenformen.

Alle sezernierenden Zellen sind an der angewachsenen (inneren) Seite von einer durch deutliche Kontur erkennbaren Membrau begrenzt, an der freien sezernierenden (äußeren) Seite dagegen erscheinen sie mehr oder weniger offen, indem hier das aus dem Protoplasma hervorgehende Sekret sich nach außen hin entleert. Wir können daher auf so beschaffene Zellen die Membrantheorie anwenden und uns vorstellen, daß sie an der inneren Fläche von einer semipermeabeln Plasmamembran eingehüllt sind, daß aber an der äußeren Fläche diese Membran durch den chemischen Prozeß der Sekretion mehr oder weniger zerstört wird. 
Je mach dem Zustande der Ruhe und Tätigkeit zeigen auch diese Zellen verschiedenartige Formen ${ }^{1}$ ). Nehmen wir nun nach Analogie der Muskelzellen auch für diese Zellen an, daß die Membran für die positiven Ionen eines Elektrolyten durchgängig, für die negativen desselben aber undurchgängig ist, so sind die Hautund Drüseuströme damit gedeutet. Es ist daher auch untersucht worden, wie sich die Kraft dieser Ströme zur absoluten Temperatur verbält. Lesser ${ }^{2}$ ) fand, daß an der Froschbaut nur in den Grenzen von 10 bis $18^{\circ} \mathrm{C}$ die Kraft der absoluten Temperatur annähernd proportional steigt; bei höheren Temperaturen dagegen steigt die Kraft viel stärker, als dem Verhältnis zur absoluten Temperatur entspricht, und bei sehr niederen Temperaturen sinkt sie stärker. Dieses Verhalten kann mau aber nicht als Einwand gegen die Annahme einer Konzentrationskette in den Drüsenzellen benutzen. Es erklärt sich diese Abweichung vom Temperaturgesetz sehr wohl aus der Empfindlichkeit der Plasmamembran gegen zul hohe und zu niedere Temperatur, wie dies auch am Muskel und Nerv, wenn auch in viel geringerem Maße bemerklich war, so dal.j nur bei mittlerer Temperatur das Gesetz zum Vorschein kommt. Die größere Empfindlichkeit des Protoplasmas dieser Zellen gegen Änderungen der Temperatur hängt wohl damit zusammen, dab ihre Funktion ja wesentlich in der Erzeugung chemischer Produkte besteht, worauf die Temperatur einen so hohen Einfluf besitzt, da nach dem van't Hoffschen Gesetz sich diese Prozesse ron $10 \mathrm{zu} 10^{\circ} \mathrm{C}$ um das Zwei- bis Dreifache beschleunigen.

Feruer sind viele Versuche über die Einwirkung der Nervenerregung an der Froschbaut angestellt worden. Ältere Versuche (Röber) ergaben eine negative Schwankung des Hautstromes

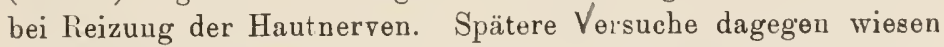
nach, daß dieser negativen häufig eine positive Schwankung folgt (Engelnann, Hermann u. a.). Wenn der einsteigende Ruhestrom stark ist, so tritt bei der Reizung eine größere negative Schwankung eiu, wenn derselbe aber schwach ist, so erfolgt eine größere positive Schwankung. An der Haut der Pfote ron Katzen,

1) Man unterscheidet häufig an diesen Zellen zwei Schichten, eine innere (angewachsene Seite) protoplasmatisclie oder homogene, in welcher der Zellkern liegt, und eine äußere (freie Seite) mehr körnige oder schleimige, welche das Sekret bildet.

2) Habilitationsschrift, Halle 1906; Ptlügers Arch. 116 (1907). 
welche bei Nervenerregung Schweißsekretion erkennen läßt, haben Hermann und Luchsinger nur positive Schwankung des Stromes beobachtet.

Eine Verstärkung des einsteigenden Stromes, die positive Schwankung, würdeu wir bei der Sekretion in folgender Weise erklären können. Während der Ruhe wirkt die Membran der Außenseite der der Innenseite entgegen, bei der Tätigkeit aber wird durch den chemischen Prozeß die Membran der Außenseite zerstört, und nun kommt die Kraft der Innenseite zur stärkeren Geltung, um so stärker, je schwächer der Ruhestrom war. Die negative Schwankung dagegen ist im Zusammenhang mit der Sekretion schwer zu deuten, man müßte denn annehmen, daß die Permeabilität der Membran der Innenseite für das Anion (- - Ion) zunimmt.

Eine ganz andere Deutung der negativen Schwankung bei der Reizung ist folgende. Ich habe beobachtet, daß, wenn man die Froschhaut über ein offenes Glasrohr spannt, dasselbe mit physiologischer Kochsalzlösung füllt, in ein Gefäß mit dieser Lösung taucht und von beiden zum Galvanometer leitet, ein Streichen der äußeren Hautfläche mit einem feinen Haarpinsel immer eine negative Schwankung des Hautstromes hervorruft. Dieselbe tritt nicht ein, wenn man die innere Fläche streicht. Es ist klar, daß es sich bei dieser schwachen Reizung um Empfindungs-bzw. Tastnervenorgane handelt und nicht un Sekretionszellen. Bei der Reizung der Hautnerven werden aber auch die Fasern dieser Empfindungsnerven gereizt, und es ist also möglich, daß die negative Schwankung des Hautstromes, die auch immer der positiven vorausgeht, nur auf Reaktion der Endigung dieser Nerven in der Haut beruht. Ist der Sekretionsstrom schon von vornherein stark, so kommt diese negative Schwankung besser zum Vorschein.

In neuerer Zeit hat Orbelli bei Garten ') auch die Froschhautströme mit dem Saitengalvanometer aufgenommen. Er findet beim Eintauchen der Haut in destilliertes Wasser einen schwachen Ruhestrom und starke positive Schwankung, beim Eintauchen in 0,6 proz. $\mathrm{Cl} \mathrm{Na-Lösung} \mathrm{einen} \mathrm{starken} \mathrm{Ruhestrom} \mathrm{und} \mathrm{negative}$ Schwankung vor.

Was die Schleimbaut des Darmkanals anbetrifft, so hat man, wie ich meine, bei der weiteren Untersuchung ihrer Ströme fol-

1) Siehe Garten, Handb. d. vergl. Physiol, 3, 156. 
gende wesentliche Tatsache zu beachten. Die Zellen derselben, welche die Oberfläche auskleiden, siud ihrer Funktion nach keineswegs, wie die der Drüsen der Schleimhaut, nur als sekretorische anzusehen, sondern die Mehrzahl derselben, besonders die Epithelzellen des Dünndarms und seiner Zotten, sind als Resorptions. zellen zu betrachten. Während bei den sekretorischen Zellen der Flüssigkeitsstrom von innen nach außen gerichtet ist, geht derselbe bei den Resorptionszellen während ihrer Tätigkeit von außen nach innen. In welchem Zusammenhang nun auch dieser Flüssigkeitsstrom mit den elektrischen Erscheinungen dieser Häute stehen mag, es ist hiernach doch wahrscheinlich, daß auch die Resorptionszellen ein elektrisches Potential besitzen, welches dem der Sekretionszellen entgegengesetzt sein kann. Diese Hypothese ist vielleicht geeignet, manche verworrene und sich widersprechende

Fig. 51.

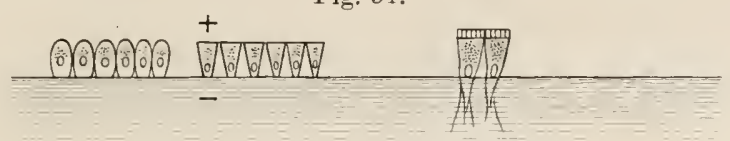

Haut mit Resorptionszellen (Schema).

Resultate in den bisher angestellten Versuchen zu klären. Auch die äufere Haut der nackten Amphibien ist nicht nur als sekretorische, sondern auch als resorbierende anzusehen. An ihr beobachtet man auch eine Umkehr des Stromes bei Erwärmen auf $50^{\circ} \mathrm{C}$ (Bach und $\mathrm{Oehler}$ ). Ferner hat Orbelli nach Eintauchen der Froschhaut in destilliertes Wasser oder sehr verdünnte Kochsalzlösungen einen umgekehrten (aussteigenden) Strom beobachtet. In dem letzteren Falle wird vielleicht eine Wasserresorption infolge von Osmose angeregt und dadurch der Strom der Resorptionszellen verstärkt. Es müßten zur Entscheidung der vorliegenden Frage Häute antersucht werden, welche nur resorbierende, aber keine sezernierende Funktion besäßen. Es scheint aber schwierig, geeignete Objekte hierza zu finden. Um der gemachten Hypothese eine anschauliche Unterlage zu geben, sei in Fig. 51 eine resorbierende Hant, mit Zellen bedeckt, schematisch dargestellt. Von den Epithelzellen des Dünndarms wissen wir, daß sie der Resorption dienen und die Spaltungsprodukte der Fette and Eiweißkörper, wie die Kohlehydrate durch ibren Zellenleib in die Lymphe und Blutbahn befördern. Die Spaltungs- 
produkte der Fette werden in diesen Zellen wieder zu Fett in feinen Fetttröpfchen vereinigt, welche in die Gewebslücken hinein ausgeschieden und von dort der Lymphbahn zugeführt werden. Die äußere Fläche dieser Zellen, welche von einer festen gestrichelten Membran bedeckt ist, läßt nur gelöste Substanzen eintreten, die innere Fläche dagegen verhält sich ähnlich wie eine äußere der Sekretionszellen. Es liegt daher die Möglichkeit vor, daß diese Zellen eine semipermeable Membran besitzen, deren äußerer Teil elektromotorisch wirkt, daf dagegen ihr inuerer Teil bei der Tätigkeit durch Abscheidung von Substanzen mehr oder weniger an elektromotorischer liraft verliert. Dadurch würde ein aussteigender Strom entstehen.

In den Sekretions- und Resorptionszellen gehen mannigfache chemische Prozesse vor sich, durch welche verschiedenartige Sekretbestandterle und Zellprodukte gebildet werden. Neben diesen chemischen Prozessen gehen aber osmotische Prozesse vor sich, welche die Aufgabe haben, Wasser von der einen Seite nach der anderen zu treiben, aus dem Blute und den Säften abzuscheiden und in das Blut und die Säfte aufzunehmen. Man hat sich vielfach mit der Frage nach den Kräften der Wasserbewegung bei diesen Vorgängen beschäftigt. Nach den bisherigen Untersuchungen scheint es nicht möglisch, diese Wasserbewegung allein aus den Gesetzen der Diffusion und denen des osmotischen Druckes zu erklären, obgleich diese Kräfte von entschiedener Bedentung hierbei sind. Man findet sehr häufig, daß der osmotische Druck der Sekrete, des Harns, der Galle, des Speichels usw., niedriger sein kann, als der des Blutes, und daf die Filtrationskraft des Blutdruckes nicht ausreicht, um diesen Unterschied zu erklären. Andererseits hat man auch beobachtet, daß aus dem Darm Flüssigkeiten resorbiert werden, welche den gleichen oder sogar höheren osmotischen Druck besitzen als das Blut. Wenn auch manche dieser Vorgänge bei der Kompliziertheit der Bedingungen im lebeuden Organisinus sich aus osmotischen Gesetzen erklären könnten, so hat man doch noch an andere Kräfte gedacht, welche die Wasserbewegung in den Zellen beherrschen könuten. Lehnt man hier Kräfte vitaler Natur von vornherein ab, so bleibt wohl nichts anderes übrig, als elektrische zu Hilfe zu nehmen ${ }^{1}$ ).

$\left.{ }^{1}\right)$ Siehe Bernstein, Lehrb.d. Phys., 3. Aufl. 1910, S. 161, 200 ff., 706. 
Es ist daher in diesem Siune von großem Interesse, die Erscheinungen der Elektroosmose zu betrachten. Von G. Wi edeman $\mathrm{n}$ ist dieselbe mit Hilfe des in Fig. 52 abgebildeten Apparates genauer untersucht worden. Ein unten geschlossener Tonzylinder $a$ ist oben mit einer Glocke und Rohr $d$ mit Seitenrobr $e$ versehen. Eine Elektrode $c$ ist luftdicht in den Zylinder eingesetzt, eine zweite $i$ befindet sich in dem äußeren Gefäß h. Füllt man den Apparat mit Flüssigkeit, so kann man durch einen Strom von $k$ nach $f$ die Flüssigkeit durch die Tonzelle hindurchtreiben und in $l$ sammeln. Der Versuch kann mit verschiedenen Flüssigkeiten vorgenommen werden, z. B. mit Wrasser und Platinelektroden oder mit $\mathrm{Cu} \mathrm{SO}_{4}$ Lösung und $\mathrm{Cu}$-Elektroden. Es ergeben sich folgende Gesetze:

"Die Menge der in gleichen Zeiten durch die Tonwand übergeführten Flüssigkeit ist der Intensität des Stromes proportional und unter sonst gleichen Bedingungen von der Oberfläche und Dicke der Tonwand unablıängig." Wenn man

Fig. 52.

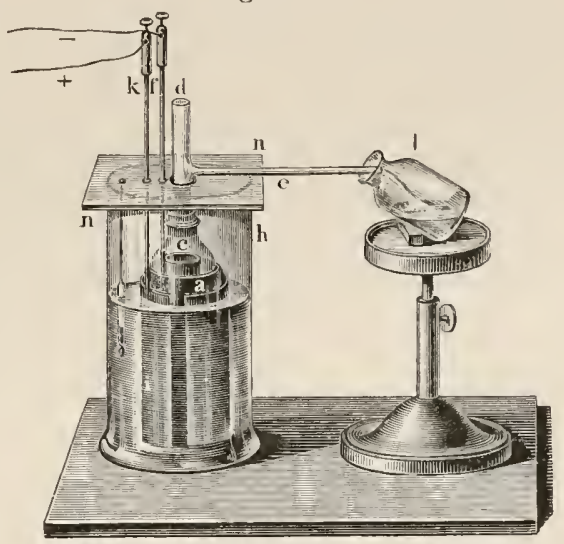

Apparat von G. Wiedemann zur Elektroosmose.

an das Seitenrohr $e$ ein Quecksilbermanometer auschließt, so kann man die Kräfte messen, welche durch Elektroosmose entsteben. Wiedemann fand: "Die Druckhöhen, bis zu welchen die Flüssigkeiten durch den galvanischen Strom ansteigen, sind der Intensität des Stromes direkt, der freien Oberfläche des Tonzylinders umgekehrt proportional."

Es ist ferner von Quincke gezeigt worden, daß sich der Vorgang auch umkehren läßt, d. h. wenn man durch poröse Scheidewände Flüssigkeit durch Druck bindurchpreßst, so entstehen dadurcb Ströme, welche man Strömungsströme genannthat. Endlich hat man auch beobachtet, daß fein verteilte Partikelchen, Schwefel, Kuble usw., sich in einer Flüssigkeit durch den Strom 
nach der Anode oder Kathode hin bewegen. Diese Erscheiuungen, welche man auch an Kolloidpartikelchen, Zellen und einzelligen Organismen (Bakterien usw.) wahrgenommen hat, hat wan mit dem Namen Elektrokinese (Freundlich) bezeichnet.

Alle diese Vorgänge haben eine gemeinsame Ursache. Von Helmboltz ist zuerst eine Theorie der Elektroosmose gegeben worden. Diese Theorio geht von der Vorstellung aus, daß ein Kontaktpotential zwischen den Wasserteilchen und der Wandung der Diaphragmaporen oder eines Rohres entsteht. Dieses Kontaktpotential kann man jetzt als ein Adsorptionspotential ansehen, indem man annimnt, daß $\mathrm{H}^{+}$- und $\mathrm{OH}^{-}$- Ionen in verschieden starkem Grade adsorbiert werden (Freundlich). Die Wasserteilchen in der Wandschicht werden dadurch positiv geladen, und wenn ein Strom hindurchgeleitet wird, so werden sie mit der positiven Elektrizität in der Richtung des Stromes mitgenommen. Infolge der inneren Reibung der Flüssigkeit werden die benachbarten inneren Schichten der Flüssigkeit mitbewegt, und in engen Röhren und den Kapillaren eines Diaphragmas erstreckt sich diese Nitbewegung daher auf den ganzen Querschnitt derselben. Die Kiraft, mit welcher diese Elektroosmose vor sich geht, ist propor"tional dem Potentialsprung $\varepsilon$ an der Grenze der Flüssigkeit und der festen Substanz des Diaphragmas, proportional dem Potentialgefälle $H$ des zugeleiteten Stromes im Diaphragma und proportional der Dielektrizitätskonstante $D$ der Flüssigkeit, dagegen umgekehrt proportional der inneren Reibungskonstante $\eta$ der Flüssigkeit. Die von $\mathrm{Per} \mathrm{rin}^{1}$ ) etwas vereinfachte $\mathrm{Helmholtzsche} \mathrm{Formel} \mathrm{für}$ die in der Zeiteinheit durch Osmose übergeführte Flüssigkeitsmenge $v$ ist bei dem Quer'schnitt $q$ :

$$
v=\frac{q \cdot \varepsilon \cdot H \cdot D}{4 \pi \cdot \eta}
$$

Dieselbe Theorie erklärt auch die Entstehung eines Strömungsstromes beim Hindurchpresseu von Flüssigkeit durch ein Diaphragma.

Ebenso ergibt sich aus ihr die Erscheinung der Elektrokinese. Es entsteht ein Kontaktpotential zwischen den suspendierten Partikelchen und dem Wasser, und wenu nun das Wasser

$\left.{ }^{1}\right)$ Siehe Freundlich, Kapillarchemie 1909, S. 222. 
in der einen Richtung bewegt wird, so werden die Partikelchen in der entgegengesetzten Richtung getrieben. Daher wandern dieselben in den meisten Flüssigkeiten zur Anode, während das Wasser zur Kathode wandert. Die Geschwindigkeit, mit welcher sich die Teilchen bewegen, ist innerhalb gewisser Grenzen nabezu unabhängig von ihrer Größe und wird durch die Formel ausgedrückt:

$$
u=\frac{\varepsilon \cdot H \cdot D}{4 \pi \cdot \eta}
$$

Die Potentialspannung $\varepsilon$ an der Grenze von Wasser und fester Substanz kann mehrere Zentivolt betragen. Die Geschwindigkeit $u$ der suspendierton Teilchen beträgt bei einem Potentialgefälle $H$ von 1 Volt auf $1 \mathrm{~cm}$ etwa 20 bis $40 \cdot 10^{-5} \mathrm{~cm}$ in der Sekunde.

Von großem Einfluß auf die Potentialdifferenz zwischen Wasser und fester Substanz ist nun die Gegenwart von Elektrolyten schon in kleinen Mengen. Durch ein Diaphragma ron Karborund (Kohlefilter) geht Wasser zur Kathode, und diese kathodische Bewegung wird durch Zusatz von Alkali beschleunigt, dagegen durch Zusatz von Säure verzögert und schließlich umgekehrt(Perrin). Zuerst ist die feste Substanz des Diaphragmas negativ gegen Wasser, und diese Ladung wird durch Alkali, und zwar durch die $\mathrm{OH}^{-}$-Ionen desselben erhöht, dagegen durch Säure, und zwar durch die $\mathrm{H}^{+}$-Ionen derselben vermindert und umgekehrt. Durch ein Diaphragma von Chromchlorid geht Wasser zur Anode, da es sich gegen die feste Substanz negativ verhält. Zusatz von Alkali hemmt und Zusatz von Säure beschleunigt hier diese Elektroosmose. Perrin hat auch die Wirkung von Salzen untersucht. Bei einem Diaphragma aus Chromchlorid, welches positiv gegen Wasser oder sehr verdünnte $\mathrm{HCl}-\mathrm{Lösung}$ ist, tritt eine Entladung durch Zusatz von $\mathrm{BrK}, \mathrm{MgSO}_{4}$ und $\mathrm{K}_{3} \mathrm{Fe}(\mathrm{CN})_{6}$ ein, und zwar durch die Anionen $\mathrm{Br}^{-}, \mathrm{SO}_{4}=$ und $\mathrm{Fe}(\mathrm{C} \mathrm{N})_{6} \equiv$ in wachsendem Maße mit ibrer zunehmenden Wertigkeit, wälırend die Kationen wenig Einfluß haben. Die zur Anode übergeführte Flüssigkeitsmenge vermindert sich in diesem Falle zunehmend in sehr beträchtlichem Grade. Bei einer negativen Diaphragmasubstanz verhält es sich umgekehrt.

Versuchen wir nun diese Kienntnisse auf die Vorgänge der Sekretion bzw. Resorption anzuwenden, so bat man schon seit längerer Zeit daran gerlacht, daß die Sekretflüssigkeit durch elek- 
trische Kräfte nebeu denen der Filtration und Osmose bewegt werde. In der Tat hat der oben beschriebene Wiedemannsche Versuch äußerlich große Ähnlichkeit mit einem Sekretionsversuch an einer Drüse, z. B. der Speicheldrüse, wenn man eine Kanüle in ihren Ausfülırungsgang einlegt und den Sekretionsnerven, z. B. den Nervus lingualis, reizt. Es tropft daun in beschleunigtem Maße der Speichel ans dem Speichelgang ab. Bisher gelang es aber nicht, eine befriedigende Theorie einer Elektroosmose bei der Sekretion aufzustellen, denn man ging immer dabei von

Fig. 53.

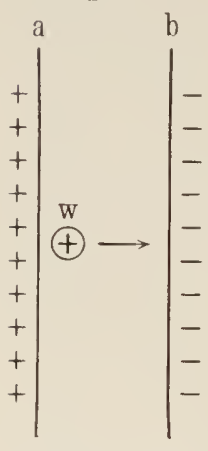

Schema zur elektroosmotischen Membrantheorie von Bernstein. der Vorstellung aus, daß die an den Häuten und Drüsen beobachteten Ströme es seien, welche die treibende Kraft gäben. Diese Ströme siud aber er'stens so schwach, daß sie gar keine merklich IVirkung ausüben könnten, und dann müßte ein solcher Strom, wenu er vorhanden wäre, doch in der Haut selbst eine Rückleitung haben, in welcher die zu bewegende Flüssigkeit wieder rückwärts getrieben würde. Ein solcher geschlossener Leitungsstrom ist aber offenbar gar nicht vorhanden; denn stellen wir uns, wie in Fig. 50, eine kontinuierliche Fläche von dicht aneinander steheuden Sekretionszellen annähernd gleicher Kraft vor, so haben wir es hier mit einer ungescblossenen elektrischen Doppelschicht $\mathrm{zu}$ tun. In dieser kann also ein Leitungsstrom nicht zustande kommen.

Die auf Grund dieser Betrachtung von mir anfgestellte Theorie der Elektroosmose in den Sekretionszellen ist nun folgende:

Es sei in Fig. $53 a$ die positive und $b$ die negative Fläche der Membran und $w$ ein Wassermolekül in der Näbe der positiven Fläche, welches gegen die Substanz der Membran positive Spannung angenommen hat. Dieses Molekül wird daher von der positiven Fläche $a$ abgestoßen, von der negativen $b$ angezogen und so durch die treibende Kraft des Potentialgefälles durch die Membran hindurchgetrieben werden. Die negative Elektrizitätsmenge der Membranteilchen, welche von der Ladung gegen die Wassermoleküle herrührt, wird an die positive Ladung in $a$ abgegeben, und die positive Elektrizität der Wassermoleküle wird an die negative in l, gebunden, so dals die Wasser- 
moleküle elektrisch neutral ans der Nembran heraustreten; d. h. es wird durch Konvektion positive Elektrizität von der Seite a nach der Seite $b$ befördert. Indem Wassermoleküle in derselben Richtung nachströmen, entsteht dadurch eine Elektroosmose. Jede Sekretionszelle kann auf diese Weise Wasser aus der inneren Seite, d. h. aus der Gewebsflüssigkeit in ihr Inneres und von da nach außen hin befördern.

Nach dieser Theorie also ist es nicht ein Leitungsstrom, sondern ein Konvektionsstrom, durch welchen außer durch die Kräfte der Osmose und Filtration die Sekretion des Wassers in den Sekretionszellen unterhalten wird. Ebenso kann auch bei der Resorption eine Wasserbewegung in den Resorptionszellen in der entgegengesetzten Richtung von auben nach innen in das Gewebe geschehen. Die Potentiale, welche hierzu erforderlich sind, brauchen keineswegs so grole zu sein, wie wir sie in den Experimenten über Elektroosmose anwenden müssen, es kommt vielmehr hauptsächlich, wie die Formel (19) lehrt, auf das Potentialgefälle $H$ an, und dieses ist trotz des geringen Potentialwertes $\varepsilon$ in der Plasmamembran ein außerordentlich großes, weil die Dicke der Membran eine außerordentlich kleine ist. Der entsprechende Konvektionsstrom hat die umgekehrte Richtung wie die abgeleiteten Ströme. Mit dieser Theorie ist die große Schwierigkeit gehoben, welche sich bisher der Annahme solcher elektrischen Triebkräfte bei der Sekretion und Resorption entgegenstellte.

Man wird vom energetischen Gesichtspunkte aus sofort die Frage aufwerfen, welche Energiequelle die hierzu nötige elektrische Energie liefere. Da die Elektroosmose des Wassers elektrische Energie verbraucht und diese aus der osmotischen Energie des Elektrolyten bezieht, welcher die Doppelschicht erzengt, so muß dieselbe aus der Osmose des Elektrolyten beständig ersetzt werden, und dies geschieht bekanntlich unter Verbrauch von Wärme. Dieser Prozeß ist also als ein endothermer anzusehen. Die Energiequelle ist Wärme des lebenden Gewebes, des Blutes und der chemischen Prozesse, welche mit der Sekretion verbunden sind. Die Sekretionszellen erzeugen so viel Wärme im Überschuß, daß sie wohl hauptsächlich die zur Elektroosmose nötige IVärmemenge allein liefern.

Bernstein, Elektrobiologie. 
Da bei diesem Vorgange das Potential der Membran durch Verbrauch von Energie zunächst abnehmen wird, so erklärt sich darans vielleicht die initiale negative Schwankung der Drüsenströme, die häufig auftritt. Die darauf folgende oft sehr starke positive Schwankung würde aus der stärkeren Zerstörung der äußeren sezernierenden Membrauseite der Zellen folgen, welche mit den inneren chemischen Prozessen verknüpft ist. Indem wir dieselben Eigenschaften auch den Resorptionszellen zuerteilen, so können wir elektrische Differenzen in ihnen in derselben Weise annehmen, aber in umgekehrter Richtung, und so kann an Häuten, wie an der Schleimhaut des Darmkanals, die elektrische Reaktion bei der Sekretion und Resorption sich in mannigfacher Weise komplizieren. Hierzu kommt noch, daß auch die chemische Reaktion des Sekretes einen Einfluß auf Richtung nnd Stärke des Drüsenstromes baben wird. Fortgesetzte Untersuchungen von diesem Gesichtspunkte aus dürften, wie ich vermute, geeignet sein, in diesem Gebiete zu weiteren Résultaten zu führen.

Um diese elektroosmotische Membrantheorie, wie ich dieselbe nennen möchte, durch Versuche zu stützen, wurden folgende Versuche angestellt. Wenn es richtig ist, daß eine semipermeable Membran, welche eine elektrische Doppelschicht erzeugt, imstande ist, Wasser von der einen nach der anderen Seite hindurchzutreiben, so muß sich dies auch an künstlichen semipermeabeln Membranen, z. B. an der Pfefferschen Ferrocyankupfermembran, nachweisen lassen. Es wurden daher an Tonzellen, welche nach der P f ef f er schen Methode mit einer solchen Membran versehen waren, Versuche dieser Art angestelit ${ }^{1}$ ). Nachlem auf der Innenfläche einer kleinen Tonzelle die Membran nach den Angaben von Pfeffer hergestellt war, wurde sie innen mit einer 0,5 proz. Lösung von $\mathrm{K}_{4} \mathrm{FeCy}_{6}$ gefüllt, mit einem offenen $\mathrm{U}$-förmigen dünnen Manometerrohr durch einen Stopfen dicht verbunden und in eine 0,85 bis 0,825 proz. Lösung von $\mathrm{Cn} \mathrm{SO}_{4}$ hineingestellt. Die $\mathrm{CuSO}_{4}$-Lösung hatte einen um ein geringes höheren osmotischen Druck als die $\mathrm{K}_{4} \mathrm{FeCy}_{6}$-Lösung, so daß vermöge der Diffusion Wasser von innen nach außen hatte waudern müssen. Der

$\left.{ }^{1}\right)$ Diese Versuche sind zum Teil gemeinsam mit Fräulein Elis a be th $\mathrm{Krösing}$ in den Jahren 1905/06 im physiologischen Institut in Halle a. S. ausgeführt worden. Dieselben sollen demnächst ausführlicher veröffentlicht werden. 
osmotische Druck wurde nach der Gefrierpunktsmethode bestimmt. Nichtsdestoweniger war in dem Manometer der Tonzelle sehr bald eine deutliche Drucksteigerung zu beobachten, wie folgende Beispiele zeigen:

Versuch 1.

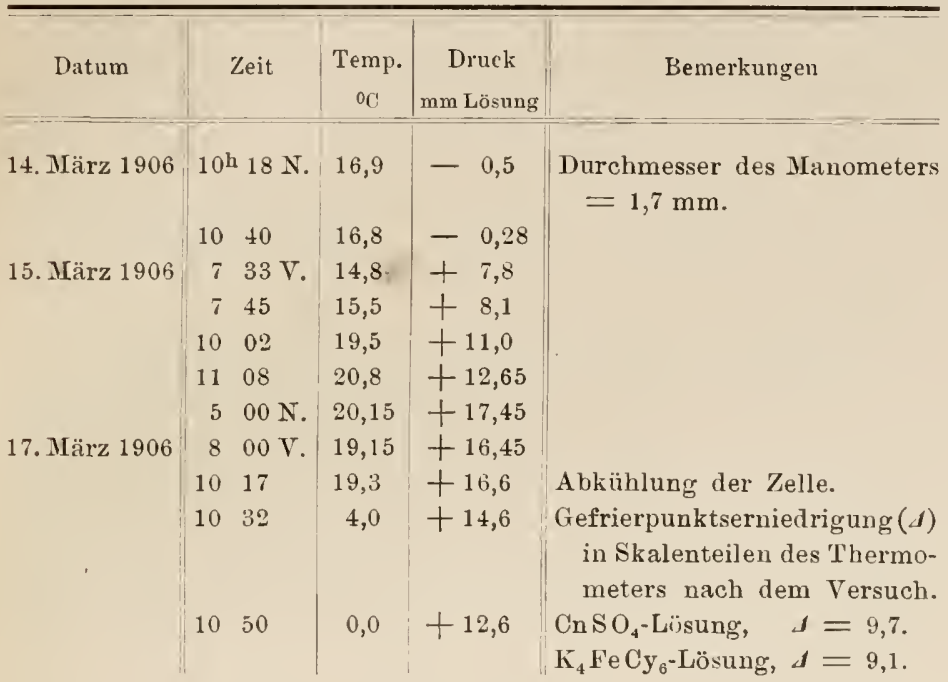

Versuch 2.

\begin{tabular}{|c|c|c|c|c|}
\hline Datum & Zeit & $\begin{array}{c}\text { Temp. } \\
\text { OC }\end{array}$ & $\begin{array}{c}\text { Druck } \\
\text { mm Lösung }\end{array}$ & Bemerkungen \\
\hline \multirow[t]{5}{*}{ 14. März 1906} & $1^{\text {h }} 00 \mathrm{~N}$. & 18,2 & $-0,7$ & Manometer $1,7 \mathrm{~mm}$ weit. \\
\hline & 204 & 18,2 & $+1,7$ & \\
\hline & 345 & 18,9 & $+5,9$ & \\
\hline & 830 & 17,2 & $+17,2$ & \\
\hline & $10 \quad 40$ & 16,8 & $+20,4$ & \\
\hline \multirow[t]{4}{*}{ 15. März 1906} & $73.3 \mathrm{~V}$. & 14,8 & $+27,3$ & \\
\hline & $10 \quad 57$ & 3,8 & $+24,7$ & Abkühlung. \\
\hline & $1207 \mathrm{~N}$. & 1,2 & $+23,4$ & \\
\hline & $12 \quad 52$ & 0,0 & $+22,2$ & $\begin{array}{c}0,825 \text { proz. } \mathrm{CuS} \mathrm{O}_{4}, d=10,4 . \\
0,5 \text { proz. } \mathrm{K}_{4} \mathrm{Fe}_{6}, d=8,9 . \\
11 *\end{array}$ \\
\hline
\end{tabular}


Diese Versuche zeigen ein deutliches Steigen der Flüssigkeit auf der Seite der $\mathrm{K}_{4} \mathrm{FeCy}_{6}$-Lösung, trotzdem diese einen etwas geringeren osmotischen Druck besitzt als die Kupferlösung. Dies konnte also nur die Folge oiner Potentialdifferenz an beiden Seiten der Membran sein. Das Kaliumion auf der Außenseite und das nicht durchgelassene $\mathrm{F}_{\theta} \mathrm{Cy}_{6}$-Ion auf der Innenseite der Membran erzeugen ein starkes Potentialgefälle in derselben, welches die Wassermoleküle von außeu in die Zelle hineintreibt. Da der osmotische Druck der Lösungen bei $0^{0}$ bestimmt wird und der der $\mathrm{K}_{4} \mathrm{FeCy}_{6}$-Lösung sich vielleicht bei mittlerer Temperatur etwas stärker erböhen könnte als der der Kupferlösung, so wurde die Zelle auch auf $0^{0}$ abgeküblt. Hierbei sank zwar der Druck im Manometer, vornehmlich infolge der Volumabnahme, weniger durch Abnahme des Potentials, aber er blieb noch hocb genug stehen.

Ein noch viel böherer Druck konnte auf der Innenseite der Zelle beobachtet werden, wenn man vorher eino stärkere Membrau nach den Angaben von Pfeffer mit 3 proz. Lösungen beiderseits erzeugt hatte. Nachdem diese mehrere Tage vorbereitet war, wurde sie innen mit 0,4 proz. $\mathrm{K}_{4} \mathrm{Fe}_{e} \mathrm{Cy}_{6}$-Lösung mehrfach gespült und in eine mehrfach erneuerte 0,825 proz. $\mathrm{CuSO}_{4}$-Lösung hineingestellt. Der osmotische Druck der ersteren ist merklich geringer als der der letzteren.

Versuch 3 .

\begin{tabular}{|c|c|c|c|c|}
\hline Datum & Zeit & $\begin{array}{l}\text { Temp. } \\
{ }^{\circ} \mathrm{C}\end{array}$ & $\begin{array}{c}\text { Druck } \\
\text { mm Lösung }\end{array}$ & Bemerkungen \\
\hline 1. Dez. 1906 & $12^{\mathrm{h}} 10 \mathrm{~N}$. & 21,5 & 30 & $\begin{array}{l}\text { Jed. Tag wurden die Liösungen } \\
\text { erneuert. }\end{array}$ \\
\hline 2. Dez. 1906 & 12 & - & $>340 !$ & $\begin{array}{l}\text { Die Flüssigkeit tropft über } \\
\text { dem Manometerrohr aus. }\end{array}$ \\
\hline \multirow[t]{3}{*}{ 8. Dez. 1906} & 11 & 19,0 & 一 & Abkühlung. \\
\hline & 12 & 1,5 & $>340$ & \\
\hline & 110 & 0,0 & $>340$ & . \\
\hline
\end{tabular}

Durch diese Versuche ist also eine neue elektroosmotische (oder kataphoretische) Eigenschaft semipermeabler Membranen festgestellt. Auch wenn wir nicht der Ostwaldschen, 
sondern der Nernst-Riesenfeldschen Anschauung über das Verhalten einer solchen Membran als Phase bzw. Lösungsmittel (s. oben S. 37) folgen, so werden wir darauf dieselbe Betrachtung auwenden und einer solchen Membran eine Wasser befördernde Kraft zuerteilen dürfen.

\section{Neuntes Kapitel.}

\section{Ursprïngliche physiologische Bedeutung des Membranpotentials der Zellen.}

\section{Die Wasserbindung in den Zellen. Genese der elektrischen Eigenschaften der Organe. Die Pflanzenströme.}

Eine unmittelbare Konsequenz der elektroosmotischen Membrantheorie führte mich zu der Frage der Wasserbindung in den Zellen, welche mit der der Wasserbewegung im engsten Zusammenhange stebt. Man hat bisher allgemein angenommen, daß die Bindung des freien Wassers, d. h. des nicht chemisch gebundenen, in den Zellen durch den osmotischen Druck und durch Quellung kolloider Substanzen erfolge. Weun es aber richtig ist, daß, wie im vorigen Kapitel bewiesen, semipermeable Membranen und demnach die Plasmamembranen Wasser von der einen nach der anderen Seite treiben können, so muß auch der Wassergehalt und die Wasserbindung der Zellen von dem Potential ihrer Plasmamembran beeinflußt werden. Wir haben die Wasserbewegung zuerst an den Sekretions- und Resorptionszellen betrachtet, welche an einer Seite mehr oder weniger als offene anzuseben sind. Aber auch an anderen, allseitig von der Plasmamembran umschlossenen Zellen muß hiernach die Wirkung des Membranpotentials in demselben Sinne zur Geltung kommen. Nebmen wir in ihnen zunächst, wie in den Muskel-und Nervenelementen, ein Nembranpotential an, welches Wassermoleküle von außen nach innen fördert, so muß die wasserbindende Kiraft der 
lebenden Zelle nicht allein vom osmotischen Druck, sondern auch von dem Membranpotential abhängig sein. Nennen wir den osmotischen Druck außerhalb der Zelle $p_{a}$ und innerhalb $p_{i}$ und die vom Membranpotential abhängige Kraft $\pi$, so müßte im Zustande des Gleichgewichtes $p_{i}+\pi=p_{a}$ sein.

Wenn diese Voraussetzung richtig ist, so müBte die wasserbindende Kraft der lebenden Zellen und Gewebe größer sein als die der toten, und wenn wir dies beweisen können, so erhebt sich die Frage, welche Bedeutung diese Eigenschaft für das Leben der Zellen und Organismen überhaupt besitze.

Es leuchtet von vornherein ein, daß eine solche Fähigkeit, Wasser stärker zu binden und festzuhalten als tote Körper, für alle Organismen, welche an der Luft lebend der Verdunstung ausgesetzt sind, von großem Vorteil sein, aber auch für das Leben im Wasser und für das Zellenleben in den Gewebssäften von Bedeutung werden muß. Hierin wäre dann, wie ich meine, die eigentliche und ursprüngliche Bedeutung des Membranpotentials der Zellen zu suchen. Gehen wir auf eine phylogenetische und histogenetische Betrachtung ein, so hätten wir uns vorzustellen, daß diese Eigenschaft unter den Einwirkungen der Umgebung durch Anpassung und im Kampfe ums Dasein allmählich erworben wurde, zuerst bei einzelligen Organismen, und dann sich auf die mehrzelligen in verschiedenem Grade unter Differenzierung der Zellen übertragen und weiter ausgebildet habe. So mag es bei der Entstehung der Sekretionszellen und Resorptionszellen zu der wassertreibenden Wirkung des Membranpotentials gekommen sein, wie ja so häufig in der Natur mit dem Formwechsel der Zellen dieselben Kräfte einem Funktionswechsel dienen. So würden sich dann bei Entwickeluug der Muskel- und Nervenzellen und -fasern die uns nun bekannten Beziehungen ihres Membraupotentials zum Vorgange der Erregung; der Reizleitung und der Kontraktion hergestellt haben. Die an diesen Elementen vornehmlich studierten elektrischen Vorgänge, welche wir bisher nur als Zeichen innerer Prozesse aufgefaßt haben, können doch füglich nicht eine solche Bedeutung allein besitzen, sondern bedürfen einer allgemeinereu Deutung in ihrer Beziehung zum Zellenleben, welche wir hiermit gefunden zu haben glauben. Die von ihnen ableitbaren elektrischen Ströme besitzen, wie wir längst wissen, gar keinen besonderen physiologischen 
Wert. Aber schließlich hat die Natur gewissermaßen die Gelegenheit benutzt, durch Funktionswechsel aus diesen Elementen Organe zu schaffen, deren Elektrizitätsproduktion einen beträchtlichen Energiewert nach außen hin besitzt - die elektrischen Organe. Noch eine andere Beziehung elektrokinetischer Art werden wir dem Membranpotential im Zellenleben zuzuschreiben habeu. Somit hätten wir den elektrischen Eigenschaften in den Oryanismen eine allgemeinere biologische Grundlage gegeben, welche den Titel dieses Buches "Elektrobiologie" rechtfertigen möge. Dieser Gedanke soll in den Schlußbetrachtungen (s. unten) weiter ausgeführt werden.

Gehen wir nun zu den Beweisen für diese Theorie über. Zuerst wählte ich zur Untersuchung solche Gewebe, welche während des Lebeus der Wasserverdunstung ausgesetzt sind. Ich stellte daher Versuche an der Froschhaut an, welche ein sehr geeignetes Objekt hierzu ist und außerdem elektrische Potentialdifferenzen besitzt. Weun es richtig ist, daß die lebenden Zellen das Wasser fester binden als tote, so muß in dem toten Gewebe die Verdunstung unter gleichen Bedingungen, d. h. bei gleicher Temperatur und gleichem Wassergehalt der Luft schneller vor sich gehen als an dem lebenden. Es war notwendig, hierzu zwei Hautstücke von möglichst gleicher Beschaffenheit, den symmetrischen Stellen des Körpers entnommen und von gleicher Oberfläche zu gleicher Zeit an demselben Orte miteinander zu vergleichen, das eine lebend (frisch, überlehend), das andere auf irgend eine Weise abgetötet. Die Abtötung wurde meistens durch Erwärmen auf 45 bis $50^{\circ} \mathrm{C}$ in einem kleinen abgeschlossenen Röhrchen vorgenommen.

Zuerst wählte ich eine Methode, welche von dem bekaunten Liebigschen Versuche über die Imbibitionskraft von Membrauen ausging. Wenn man eine Nembran über das trichterförmig gestaltete obere Ende eines langen Rohres bindet, das Ganze mit Wasser füllt und das untere Ende des Rohres in eine Schale mit Hg einstülpt, so sieht man infolge der Verdunstung des Wassers auf der Membran das $\mathrm{Hg}$ in dem Rohre allmählich bis zu einer gewissen Höhe steigen. Diese Höhe gibt die Imbibitionskraft der Membran an.

Wcnn nun an der lebendeu Membran die Verdunstung langsamer erfolgt als an der totel, so muß eine Differenz im Steigen der Hg-Säule in diesem Versuche zu beobachten sein. Es wurden in diesen Versucben, um sie schneller zu beenden, Kapillarröhren 
verwendet und mit zwei gleich großen Trichtergefäßen verbunden. Folgendes Beispiel aus einer Reihe solcher Versuche zeigt einen sehr deutlichen Unterschied beider Membranen:

Versuch (21. MIärz 1907).

\begin{tabular}{|c|c|c|c|c|c|}
\hline \multirow{2}{*}{$\mathrm{Nr}$. } & \multirow{2}{*}{ Zeit } & \multicolumn{2}{|c|}{$\mathrm{Hg}-\mathrm{Stand}$} & \multirow{2}{*}{$\begin{array}{l}\text { Temp. } \\
{ }^{0} \mathrm{C}\end{array}$} & \multirow{2}{*}{ Bemerkungen } \\
\hline & & Leb. Haut & Tute Haut & & \\
\hline 1 & $9^{\text {hl }} 58 \mathrm{~V}$. & 0 & 0 & & \\
\hline 2 & 1158 & 0 & 0 & & Mit 0,6 proz. CINa-Lösung \\
\hline \multirow[t]{7}{*}{3} & $1^{\mathrm{h}} \mathrm{N}$. & 7 & 10 & 17 & $\begin{array}{l}\text { gefüllt. } \\
\text { Rückenhaut, Außenfläclie }\end{array}$ \\
\hline & 2 & 23 & 52 & 18 & außen. \\
\hline & 4 & 85 & 160 & 20 & . \\
\hline & 6 & 180 & 285 & 20 & \\
\hline & 8 & $25 i^{\circ}$ & 347 & 18 & \\
\hline & 10 & 307 & 375 & 16 & \\
\hline & $8^{\text {hi }} \mathrm{V}$. & 401 & 400 & 15 & \\
\hline
\end{tabular}

Eine größere Zahl von Versuchen ergab dasselbe Resultat, und es war gleichgültig, ob die Außen- oder Innenseite der Haut außeu war. Statt der Abtötung durch Erwärmen wurde auch Trocknung der Haut vurgenommen und die trockene Haut dann in (0,6 proz. Cl Na-Lösung gelegt. Das Resultat war dasselbe. Man ersieht hieraus, daß alle Zellen der IIaut, nicht nur die der Epidermis, sondern auch die der Cutis, im lebenden Zustande das Wasser gegen Verdunstung stärker festhalten, als die des toten Gewebes. Es konnte an der Hant zu gleicher Zeit die elektromotorische Kraft gemessen werden, indem man der Kochsalzlösung etwas Kalomel zusetzte und vom $\mathrm{Hg}$ der Schale und mit einer Hg-Kalomelelektrode von der Hautfläche ableitete. Die tote Haut war entweder stromlos oder schwach umgekehrt wirksam; der Strom der lebenden Haut nahm langsam ab.

Das Maximum der Hg-Höhe war nach längerer Zeit, meist 24 Stunden, in beiden Fällen nahezu gleich, nachdem die lebende Haut abgestorben war.

Es wurden alsdann weitere Untersuchungen ${ }^{1}$ ) ̈̈ber diesen Gegenstand mit Hilfe der Wage angestellt, indem zwei gleich gro@e

1) Diese Versuche wurden in den Jahren 1908 bis 1910 zum Teil gemeinsam mit cand. med. W. Lindemann ausgeführt. Auch diese sollen noch ausfülırlicher veröffentlicht werden. 
Ha u ts tücke, von denen das eine abgetötet, das andere lebend war, auf beiden Seiten der Wage aufgehängt wurden und die durch Verdunstung entstehende Gewichtsdifferenz nacb Äquilibrierung an der Skala der Wage bzw. durch Gowichtszusatz gemessen wurde. Um genau gleich große Stücke zu erhalten, wurde zuerst eine Stahlstanze von $2 \times 3 \mathrm{qcm}$ benutzt und die Hautstücke an Kokonfäden an zwei Ecken des Stückes aufgebängt. Später wurde eine kreisförmige Stanze von $2 \mathrm{~cm}$ l) urchmesser konstruiert, welche, nahe dem scharfen Rande innen mit feinen Widerhäkchen versehen war, die das auf Kork ausgestanzte Stück festhielten. Zwei gleiche Stanzen wurden an beiden Seiten der Wage mit toter und lebendel Membran aufgehängt.

Ich führe aus diesen Versuchen einige Beispiele an. In fast allen Versuchen zeigte sich eine deutliche Gewichtsdifferenz, welche angab, wieviel Wasser an der Ober-fäche der toten Membran mehr verdunstete als an der lebenden. Verglich man zwei lebende oder z wei tote Membranen in dieser Weise miteinander, so erhielt man nur sehr kleine und wechselnde Differenzen. Die Membranen wurden vor der Aufhängung zwischen Fließpapier gleichmäßig abgetrocknet. In den Tabellen ist die Zeit, die Gewichtsdifferenz der Membranen, die auf $1 \mathrm{qcm}$ berechnete Gewichtsdifferenz und die aus letzterer durch Wasserverdunstung gebundene Wärmemenge in g-cal angegeben. Diese Wärmemenge ist der Energiemenge äquivalent, mit welcher die lebenden Zellen der Membran gegenüber den toten das Wasser binden.

Versuch 1 .

\begin{tabular}{|c|c|c|c|c|}
\hline \multirow{2}{*}{ Stunden } & \multirow{2}{*}{$\mathrm{mg}$} & \multicolumn{2}{|c|}{ Auf $1 \mathrm{qcm}$} & \multirow{2}{*}{ Bemerkungen } \\
\hline & & $\mathrm{mg}$ & g-cal & \\
\hline 1 & 29,9 & 2,57 & 1,50 & \multirow[t]{3}{*}{ Froschbaut $2,5 \times 3,7 \mathrm{qcm}$} \\
\hline 2 & 57,8 & 6,25 & 3,65 & \\
\hline 3 & 82,4 & 8,91 & 5,21 & \\
\hline
\end{tabular}

Versuch 2 .

\begin{tabular}{l|l|l|l|c}
1 & 16,8 & 2,79 & 1,64 & Froschhaut $2 \times 3$ qcm. \\
2 & 33,6 & 5,60 & 3,26 & (Stanze). \\
3 & 39,0 & 6,5 & 3,8 &
\end{tabular}


Die beiden Flächen der Froschbaut unterschieden sich hierin nicht wesentlich, denn das Resultat war dasselbe, wenn man auf jede Seite der Wage zwei aufeinander gelegte Häute aufhing, die einmal mit den inneren, das andere Mal mit den äußeren Flächen sich deckten. Es geht daraus hervor, daß es sich hierbei nicht bloß um eine besondere Eigenschaft der Epidermiszellen handelt, sondern daß auch die Zellen der Cutis diese Eigenschaft besitzen.

Es lag nahe, an Pflanzenblättern einige solche Versuche anzustellen. Dazu eigneten sich die Blätter des Flieders (Syringa vulg.) sehr gut, indem zu beiden Seiten der Mittelrippe symmetrische Stücke ausgestanzt wurden, wie folgendes Beispiel zeigt.

Versuch 1 .

\begin{tabular}{c||c|c|c||c}
\hline \multirow{2}{*}{ Stunden } & \multirow{2}{*}{$\operatorname{mg}$} & \multicolumn{2}{|c||}{ Auf $1 \mathrm{qcm}$} & Bemerkungen \\
\cline { 3 - 5 } & & $\mathrm{mg}$ & $\mathrm{g}$-cal & \\
\hline 1 & 25,96 & 4,33 & 2,55 & Fliederblatt $2 \times 3 \mathrm{qcm}$ \\
2 & 31,68 & 5,28 & 3,10 & (Stanze). \\
$4^{25}$ & 44,78 & 7,47 & 4,39 &
\end{tabular}

Dieses Verhalten bestätigte sich in mehreren Versuchen. Man sieht, daß das Pflanzengewebe sich ganz ebenso verhält, wie das der Froschhaut. Für die Pflanze ist aber diese Eigenschaft, der Verdunstung und Austrocknung im lebenden Zustande stärker zu widerstehen als im toten, von ganz besonderer Bedeutung ${ }^{1}$ ).

1) In der botanischen Literatur fand ich über ähnliche Versuche folgendes vor: Hugov. Mohl (Botan. Ztg. 1847) verglich die Vertrocknung lebender und durch Hitze, Gifte oder Frost getöteter Pflanzen (Warmhauspflanzen mit dickeu Blättern). Er bestimmte den Gewichtsverlust in Prozenten des ursprünglichen Gewichtes und erhielt folgende Werte:

\begin{tabular}{l|c|c|c|}
\hline & $\begin{array}{c}\text { 1. bis 5. Tiag } \\
\text { Proz. }\end{array}$ & $\begin{array}{c}\text { 6. b1s 10. Tag } \\
\text { Proz. }\end{array}$ & \\
\hline Mittel. . $\left\{\begin{array}{c|c|c}11,4 \\
20,1\end{array}\right.$ & $\begin{array}{r}8,3 \\
13,7\end{array}$ & $\begin{array}{l}\text { Lebend } \\
\text { Erfroren }\end{array}$
\end{tabular}

Nägeli (Sitz.-Ber. d. bayer. Akademie 1861) untersuchte die Vertrockuung lebender und durch Frost abgetöteter Äpfel und Kartoffeln. Gefrorene Kartoffeln wurden in 117 Tagen, nicht gefrorene in 330 Tagen lufttrocken. Die Vorgänge bei Äpfeln wurden durch Fäuluis, bei Kartoffeln durch Keimung gestört. 
Als Schutz gegen Verdunstung dienen bei. Pflanzen freilich in bohem Grade die äußeren Schichten der Epidermis, die Cuticula und die Wachshaut, und es bedürfte spezieller Untersuchungen, wie diese sich etwa beim Absterben ändern (s. Pfeffer, Pflanzenphysiologie, 1897, Bd.I, S. 216 ff.).

Vornehmliches Interesse wird nun bei diesem Gegenstande das Muskelgewebe in Anspruch nehmen in Beziehung zu seinen elektrischen Eigenschaften. Es stellten sich aber der Untersuchung anfangs erhebliche Schwierigkeiten entgegen. Zwei gleiche Muskeln, M. gastrocnemius oder sartorius, hatten im lebenden und toten Zustande infolge der Starreverkürzung zu ungleiche Oberflächen, um miteinander verglichen werden $\mathrm{zu}$ können und gaben deshalb schwankende Resultate. Es wurden daher schließlich dünne glatte Muskeln als Membranen, und zwar von den Bauchmuskeln des Frosches der hierzu sehr geeignete Musc. abdominis transversus und obliquus (s. Ecker, Anatomie des Frosches) benutzt und auf die oben beschriebenen kreisförmigen Stanzen gespannt. Die Abtötung durfte wegen der Starreverkürzung, um Schrumpfung zu vermeiden, erst nach der Aufspannung im Ring gemacht werden. Statt der Erwärmung, wobei selbst in kleinen gescblossenen Gefäßen leicht merklicher Wasserverlust auftreten könnte, bediente ich mich der Chloroformierung in einem kleinen gedeckten Petrischälchen, während der lebende MLuskel unterdessen in einem gleichen aufbewahrt wurde. 10 Minuten Einwirkung von wenig in einem Uhrschälchen hineingebrachtem Chloroform reichten aus. Bis zur Aufhängung in der Wage und dem Beginn der Messung war jeder Geruch nach Chloroform geschwunden. Zwei lebende Muskelmembranen zeigten während mehrerer Stunden nur ganz verschwindende Gewichtsdifferenzen. Beispiele sind in umstehender Tabelle angeführt.

In allen Versuchen verdunstete von der lebenden Muskelmembran in der ersteu balben Stunde weniger Wasser als von der toten. Man kann annehmen, daß in dieser Zeit auch der dünne lebende Muskel schon fast abgestorben war. Daraus erklärt sich, daß die beobachteten Differenzen hier kleiner sind als bei der Froschhaut, welche stundenlang überlebt. Vergleicht man aber die Werte für die erste halke Stunde miteinander, so ist der Unterschied sehr viel geringer, etwa nur die Hälfte. Aus dem schnellen Absterben des dünnen der Verdunstung ausgesetzten 
Muskels exklärt es sich auch, daß, wenn man die Beobachtung weiter fortsetzt, die Differenzen zwischen beiden Muskeln oft späterhin abnehmen und zuweilen sich umkehren, indem nun die Verdunstung an dem zuletzt abgestorbenen Muskel schneller vor sich geht. Zuweilen wächst dann auch wieder die Differenz in dem ersteren Sinne. Aber daß in der ersten Zeit der lebende Muskel das Wasser länger und stärker festhält als der tote, ist in allen Versuchen konstant.

Versuch 1.

\begin{tabular}{|c|c|c|c|c|c|}
\hline \multirow{2}{*}{$\begin{array}{l}\text { Zeit } \\
\text { Min. }\end{array}$} & \multirow{2}{*}{$\mathrm{mg}$} & \multicolumn{2}{|c|}{$1 \mathrm{q} \mathrm{cm}$} & \multirow{2}{*}{$\begin{array}{l}T^{T} \\
{ }^{\circ} \mathrm{C}\end{array}$} & \multirow{2}{*}{ Bemerkungen } \\
\hline & & mg & g-cal & & \\
\hline 0 & 0 & 0 & 0 & \multirow{6}{*}{13,5} & \multirow{6}{*}{$\begin{array}{l}\text { Kreisförmige Stanze, } \\
\text { Fläche }=5,725 \mathrm{qcm} \\
\text { Muskelmembranen. }\end{array}$} \\
\hline 2 & 1,0 & 0,1747 & 0,1021 & & \\
\hline 7 & 2,5 & 0,4366 & 0,2553 & & \\
\hline 10 & 3,0 & 0,5240 & 0,3064 & & \\
\hline 15 & 3,25 & 0,5678 & 0,3320 & & \\
\hline 24 & 3,50 & 0,6114 & 0,3574 & & \\
\hline
\end{tabular}

Versuch 2.

\begin{tabular}{|c|c|c|c|c|}
\hline 0 & 0 & 0 & 0 & \\
\hline 10 & 0,96 & 0,1677 & 0,0981 & \\
\hline 15 & 1,44 & 0,2516 & 0,1471 & \\
\hline 20 & 1,44 & 0,2516 & 0,1471 & 19 \\
\hline 27 & 3,36 & 0,5870 & 0,3431 & \\
\hline
\end{tabular}

Versuch 3 .

\begin{tabular}{|c|c|c|c|c|}
\hline 0 & 0 & 0 & 0 & \\
\hline 3 & 1,68 & 0,2234 & 0,1716 & \\
\hline 5 & 2,16 & 0,3773 & 0,2206 & \\
\hline 8 & 2,64 & 0,4611 & 0,2696 & \\
\hline 13 & 2,88 & 0,5030 & 0,2941 & \\
\hline 18 & 3,36 & 0,5869 & 0,3431 & \\
\hline 23 & 3,36 & 0,5869 & 0,3431 & \\
\hline 28 & 3,60 & 0,6287 & 0,3676 & 21 \\
\hline 33 & 3,84 & 0,6707 & 0,3921 & \\
\hline
\end{tabular}

Versuche an anderen Geweben und Organen habe ich noch nicht ausgeführt. Die größte Schwierigkeit bietet immer hierbei die Herstellung gleicher Oberfläıhen, die bei kleiner Masse durch- 
aus erforderlich ist. Man könnte aber versuchen, großje und voluminöse Massen miteinander zu vergleichen, wobei die ungleiche Oherfläche keinen so großen Fehler bedingen würde.

Die angestellten Versuche geben aber schon den Beweis dafür, daßs die lebende Zelle die fundamentale Eigenschaft besitzt, vermöge einer ihr zukommenden Kraft das Wasser stärker zu binden als die tote. Es fragt sich, welche der uns bekannten Kräfte hierfür in Anspruch zu nehmen ist. Es ist klar, daß der osmotische Druck diese Kraft nicht sein kann. Die Konzentrationen der anorganischen wie der organischen Substanzen, welche sich in Lösung befinden, können beim Absterben in der Zelle nicht geringer werden, im Gegenteil, es müßte die Konzentration der organischen durch Spaltung und Oxydationen größer werden ${ }^{1}$ ). Es käme ferner die Quellung in Betracht, und man könnte meinen, daß die Kraft, mit welcher das lebende Protoplasma das Wasser durch Quellung binde, größer sei als die des toten. Überlegt man aber, welcher Anteil des Wassers es ist, der in der ersten Zeit verdunstet, so ist es nicht das durch Quellung in dem Protoplasma und dessen Gebilden gebundene, sondern das Wasser des Paraplasmas, das flüssige Lösungswasser der Salze und organischen Substanzen, welches mit der Zwischenflüssigkeit der Zellen, der Gowebsflüssigkeit, in direktem Austausch steht. Es liegt auch kein Grund zur Annahme vor, daß die Kraft der Quellung beim Absterben in den Zellen eine geringere würde, denn wir wissen, daßs dabei Gerinnungen eintreten, daß sich dabei Sole in Gele verwandeln, und es müßte dadurch die Wasserbindung durch Quellung sogar eine stärkere werden. Kurzum wir können die beobachteten Erscheinungen nicht durch Änderungen der Quellung erklären. Dagegen haben wir oben eine elektroosmotische Kraft kennen gelernt, welche semipermeabeln Membranen zukommt und imstande ist, Wasser von der einen nach der anderen Seite zu treiben und dasselbe hierdurch in der Zelle festzuhalten.

1) Man könnte daran denken, daß Gewichtsunterschiede zwischen totem und lebendem Gewebe auch durch Ausscheidung von $\mathrm{CO}_{2}$ bedingt würden, aber das müßte umgekehrt beim tierischen Gewebe eine größere Gewichtsabuahme des lebenden ergeben. Beim grïnen Pflanzengewebe könnte durch Assimilation hingegen eine Zunabme an Gewicht herbeigeführt werden, doch sind beide Werte in der Beobachtungszeit zu gering, als daß sie gegen den Wasserverlust in Betracht kämen. 
Es erscheint uns daher als eine berechtigte Hypothese, durch diese Kraft das Verhalten der lebenden Zelle gegenüber der toten in Beziehung zur Wasserbindung zu deuten, zumal sich dadurch eine große Reihe von Zellprozessen unter einem gemeinsamen Gesichtspunkt zusammenfassen läßt.

Nach der elektroosmotischen Membrantheorie regelt jede Zelle ihren Wassergehalt nicht nur vermöge des osmotischen Druckes in ihrem Inneren, sondern auch wesentlich durch die Wirkung des Potentialgefälles ihrer Plasmamembran. Diese Kraft ist bei der Sekretion und Resorption und bei der gesamten Wasserbewegung zwischen den Flüssigkeiten des Körpers und den verschiedenen Organzellen tätig, und so werden sich voraussichtlich die mannigfachen Vorgänge dieser Art, welche wir bis jetzt durch die Kräfte der gewöhnlichen Filtration und Diffusion nicht erklär'en können, deuten lassen. Ich erwähne nur die Absonderung eines sehr verdünnten Harnes, der einen viel geringeren osmotischen Druck besitzt als das Blut und einen beträchtlichen Energieaufwand erfordert. Auf diesen Gegenstand weiter einzugehen, ist hier nicht der Ort. Dagegen wollen wir hier die osmotischen Bewegungserscheinungen an Pflanzen anschließen, deren Deutung sich jetzt unmittelbar ergibt.

An gewissen Pflanzen, z. B. an der Sinnpflanze, Mimosa pudica, und an der Fliegenfalle, Dionaea muscipula, beobachtet man bekanntlich auffallende Reizbewegungen ${ }^{1}$ ). Bei Berührung und Erschütterung der Blätter dieser Pflanzen oder anderweitiger Reizung treten diese Bewegungen ein, welche im allgemeinen darin bestehen, daß sich die gegenüberstehenden Blätter oder Blatthälften mit ihren oberen Flächen zusammenlegen und größere wie kleinere Blattstiele sich senken. In Fig. 54 ist ein Zweig der Mimosa pudica abgebildet, auf der Seite $A$ in ruhender, auf der Seite $B$ in gereizter Stellung. Am Ursprung der großen und kleinen Blattstiele befinden sich Gelenke, welche die Bewegungen verursachen. Diese Gelenke bestehen aus zwei Gelenkwülsten, welche sich gegenüberstehen, der eine derselben nimmt an Volumen $a b$, während der andere sich vergrößert, wodurch der Blattstiel

$\left.{ }^{1}\right)$ Siehe Pfeffer, Pflanzenphysiologie, 1904, Bd. II, S. 433, Abschnitt III. 
nach einer Richtung bewegt wird. Diese Wülste bestehen aus saftreichen Zellen, deren Füllungsgrad, Turgor, sich schnell ver-

Fig. 54.

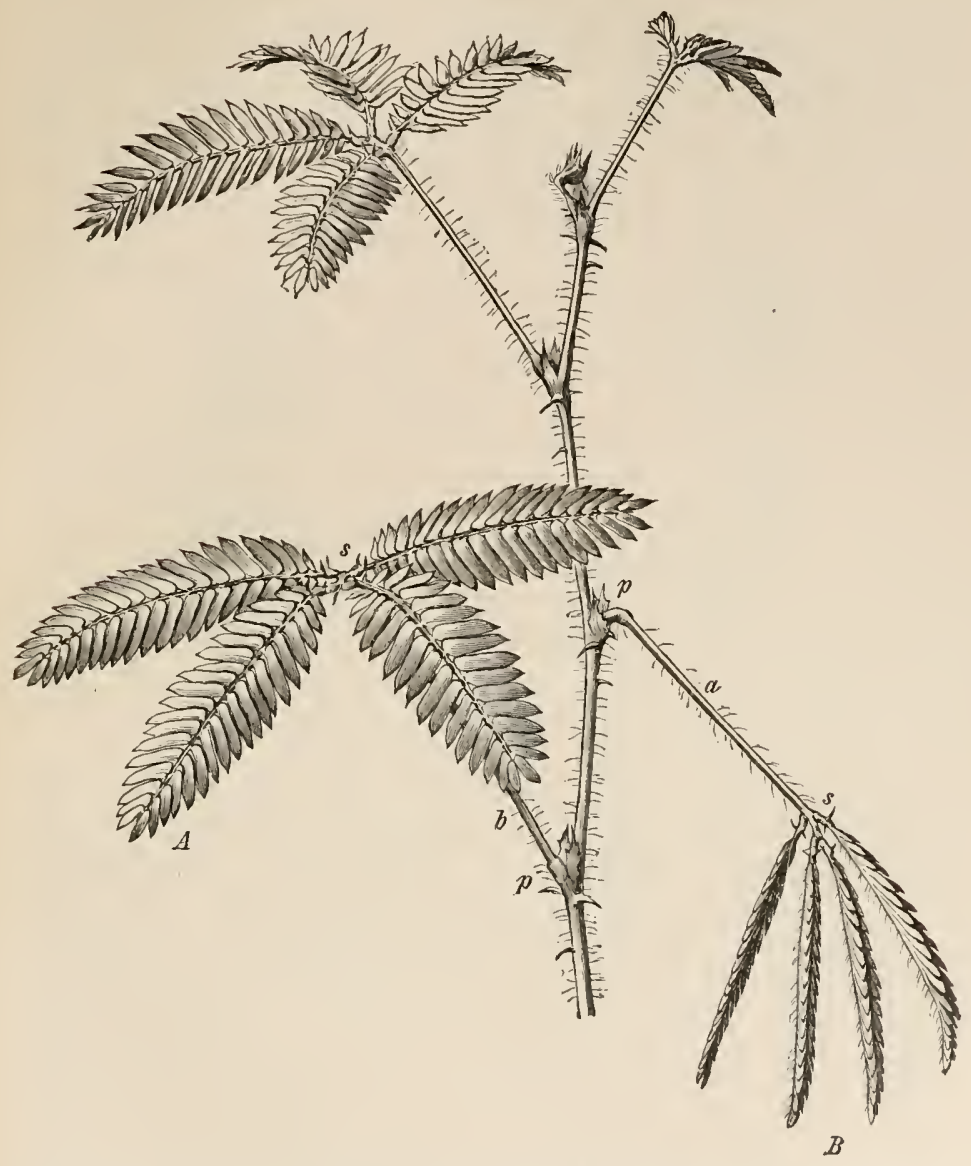

Mimosa pudica (Sinnpflanze).

Blatt $A$ in ruhender, $B$ in gereizter Stellung, $p$ das primäre Gelenk, $s$ die sekundären Gelenke an der Basis der Fiederstrahlen (nach Pfeffer, Pflanzenphysiologie II, 1909).

ändern kann. Es tritt bei der Reizung Wasser aus ihnen aus, sie ziehen sich zusammen und das Wasser fließt in interzellulare Räume und Gefäße hinein, welche zum Teil lufthaltig sind. Bei 
der Rückkehr zur Ruhestellung kehrt das Wasser auf demselben Wege in die Zellen zurück, deren Turgor wieder zunimmt. In dem Gelenk $p$ des Hauptstieles nimmt der untere Wulst an Volumen ab, der obere nimmt etwas zu, indem Wasser in dessen Interzellularraum eintritt.

Ganz ebenso ist der Mechanismus der Bewegung bei der Dionaea muscipula, deren Blatt in Fig. 55 abgebildet ist. Das Fig. 55 .

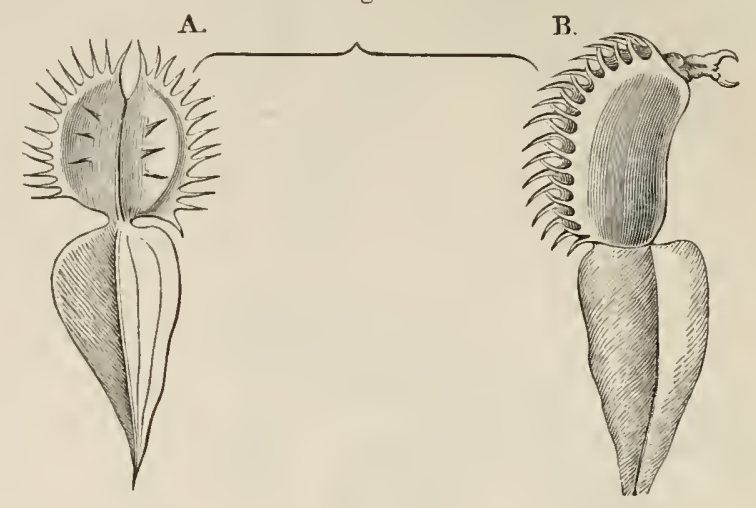

Blatt ron Dionaea muscipula (Fliegenfalle).

$A$ offen im ruhenden Zustande, $B$ geschlossen im gereizteu Zustande, ein Ohrwurm gefangen (nach Pfeffer, ebenda).

Blatt besteht aus zwei Hälften, welche sich gegeneinander wie die Schalen einer Muschel in dem Gelenk an der Mittelrippe bewegen köunen. In $A$ sehen wir das geöffnete Blatt in ruhendem Zustande, in $B$ das gereizte geschlossene Blatt, in welchem ein Ohrwurm gefangen ist. Auf der inneren Fläche des Blattes stehen drei Reizhaare, welche, in dem Gewebe eingepflanzt, durch Beugung eine Reizleitung in dem Gewebe auslösen. Die reizbaren saftigen Zellen sind namentlich in den Schichten der Innenseite gelagert, längs der Querrippen des Blattes. In der Ruhe hält die Turgorspannung dieses Gewebes der elastischen Spannung des Gewebes an der Außenseite das Gleichgewicht. Bei der Reizung erlangt letztere das Übergewicht, wodurch das Blatt sich schließt. Es sind ferner auf der. Innenfläche Drüsen vorhanden, welche einen dem Magensaft ähnlichen Verdauungssaft absondern, der durch ein Ferment Eiweiße auflöst, welche der Pflanze (fleisch- 
fressende Pflanze) zur Nahrung dienen. Ebenso findet auch bei Mimosa eine Reizleitung durch die Stiele statt, durch welche sich, ähulich wie in den Nerven der Tiere, der Reiz und die Reizbewegung ausbreitet.

Ganz ähnlich verhält sich der Vorgang an den Staubfäden gewisser Blüten, z. B. von Berberitze (Berberis vulgaris) und Blüten der Cynareen (Artischocke, Cynara scolymus). Bei diesen treten Krrümmungen und Verkürzangen bis zu 10 bis 30 Proz. der Länge bei mechanischer Reizung auf, welche in der Natur. durch Insekten geschieht, wobei sich die Staubbeutel dem Stempel nähern. Hier verkürzen sich die Zellen in der Längsrichtung durch Wasseraustritt, welcher in die Gefäßbündel hinein stattfindet (Pfeffer). Der Vorgang ist äußerlich den Muskelkontraktionen ähnlich, doch imerlich dadurch verschieden, daß das Volumen der Zellen abnimmt, während das der Muskelzelle konstant bleibt.

Es erhelut sich nun die Frage, durch welche Kraft die Wasserbewegung bei diesem Vorgange geschieht. Das ist bisher gänzlich rätselhaft geblieben ${ }^{1}$ ). Iurch die gewöhnliche Osmose läßt sich diese Wasserausscheidung und Wiederaufnahme nicht verständlich maclien, denn wie sollte in so kurzer Zeit der osmotische Druck in den Zellen sich so erheblich verkleinern und wieder vergrößern, wie es hierzu nötig wäre. Einen chemischen Prozeß in dem Zellsaft oder Protoplasma. der sich auf die darin befindlichen Salze und Kohlehydrate erstrecken müßte, zu diesem Zwecke anzunehmen, ist nicht denkbar. Es müßte ein solcher sein, der ihre Noleküle durch chemische Bindung unwirksam machte. Dagegen kenut man schon seit längerer Zeit die elektrischen Potentialänderungen in diesen die Bewegung hervorbringenden Pflanzengeweben. Besonders an der Dionaea muscipula sind Untersuchungen dieser Art von Burdon-Sanderson ${ }^{2}$ ) angestellt worden, welche ergeben haben, daß auch hier in Übereinstimmung mit den tierischen Geweben die gereizten Teile des Gewebes negative Spannung gegen die ruhenden annehmen. Ungefähr

1) Siehe Pfeffer, Pflanzenphysiolorie I, S. 252.

$\left.{ }^{2}\right)$ On the elektromotive properties of the leaf of Dionaea in excited and unexcited states. Philosophical Transactions of the Royal Society of London 1882 und 1888. (Vol. 179 B, p. 417-449.) 
0,04 Sek. nach Einwirkung eines elektrischen Induktionsschlages beginnt die elektrische Reaktion des Blattes, während die Bewegung desselben erst nach 1 Sek. einsetzt und 5 bis 6 Sek. dauert. Diese Reaktion stimmt im Prinzip mit der des Muskels vollständig überein. Die Versuche wurden mit Hilfe des Rheotoms und später des Kapillarelektrometers ausgeführt, dessen Kurven photographisch aufgenommen wurden. Man kann die Protoplasten der reizbaren Zellen, welche durch Fäden (Syndesmien) miteinander verbunden sind, als eine zusammenhängende Protoplasmamasse ausehen, in welcher sich die Bewegung fortpflanzt. Bringen wir nun die Membrantheorie auch hier zur Anwendung, so besitzt jede Zelle ein Membranpotential, welches eine gewisse Wassermenge in derselben festhält. Sinkt dieses Mem-

Fig. 56.

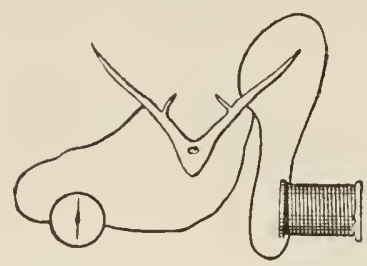

Versuch von

Burdon-Sauderson am Blatt von Dionaea muscipula. branpotential bei der Reizung, so wird die erregte Stelle, wie bei der Nerven-und Muskelfaser, negativgegen dieruhendeStelle des Gewebes, und die Folge ist nun ein Austritt des Wassers aus den Zellen in die angrenzenden Räume. Diese Wasserbewegung bedarf einer gewissen Zeit, und daher tritt die merkliche Bewegung an der Pflanze viel später auf als die elektrische Potentialschwankung.

l)er Hauptversuch von Burdou-Sanderson ist folgender. In Fig. 56 ist die Anordnung desselben schematisch abgebildet. Das geöffnete Blatt der Dionaea ist im Querschnitt dargestellt. Dasselbe ist von jeder Blatthälfte zum Galvanometer bzw. zum Kapillarelektrometer abgeleitet. Auf der einen Seite wird an der abgeleiteten Stelle aus der sekundären Spule ein Induktionsschlag zugefübrt. In der Ruhe ist nur ein schwacher oder gar kein Strom von den symmetrischen Stellen vorhanden. Nach der Reizung wird erst die gereizte Stelle negativ gegen die andere, dann nach einiger Zeit der Fortleitung auf die andere Blatthälfte diese Stelle negativ gegen die erste. In Fig. 57 sind die Kurven des Kapillarelektrometers bei der Reizung auf der einen und auf der anderen Seite wiedergegeben. Der Versuch läuft im Prinzip ganz ebenso ab wie der bei Ableitung eines Muskels oder Nerven von zwei 
Längsschnittpunkten nach lieizung wit einem Schlage (s. S. 46). Die Reizleitung in dem reizbaren Gewebe von Zelle zu Zelle findet mit einer verhältnismäßig geringen Geschwindigkeit statt, welche etwa $200 \mathrm{~mm}$ in der Sekunde beträgt.

Man hatte früher wohl daran gedacht, daß diese Ströme bei Tieizbew egungen erst die Folge derWasserverschiebung sein könnten, da bekanntlich nach den Versuchen von Quincke durch Filtration von Wasser durch Diaphragmen und Kapillaren sogenannte Strömungströme entstehen (s. oben S. 157). Aber obige Versuche haben bewiesen, daß dies nicht der Fall sein kann, da die Ströme viel früher auftreten als die Wasserverschiebung und Reizbewegung. Letztere beginnt erst nach 1 Sek., während die erste Phase des Aktionsstromes schon 0,04 Sek. nach der Reizung anhebt. Die

Fig. 57.
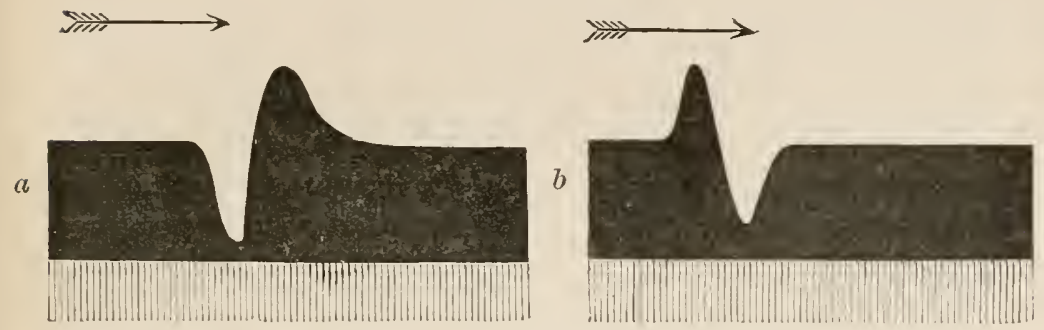

Kurven des Kapillarelektrometers im Versuch ron Burdon-Sanderson am Blatt der Dionaea. $a$ Reizung auf der einen, $b$ auf der anderen Seite, zweiphasige Aktionsströme.

Wasserbewegung kann also erst die Folge der Potentialänderungeu sein.

Auch Riuheströme sind an Pflanzen beobachtet worden. Zuerst hat L. Hermann solche Ströme an verletzten grünen Pflanzenstengeln und Pilzstielen nachgewiesen, deren Querschnitt negativ gegen die Längsoberfläche ist. Dieses Verhalten erklärt sich ebenso wie die Längsquerschnittströme der Muskeln und Nerven durch Verletzung der longitudinal geordneten Zellreihen. An unverletzten Pflanzen treten zwischen verschiedenen Teilen schwache Potentialdifferenzen auf, die ebenso zu deuten sind wie die schwachen Ströme unverletzter Muskeln. Das Membranpotential ist eben nicht an allen Stellen des zusammenhängenden Zellkomplexes diss gleiche. Eine sehr beträchtliche Potentialdifferenz bis zu 0,1 Daniell ist dagegen an keimenden Pflänzchen (Erbsen, Bohnen) gefunden 
worden, wenn man von den Wurzeln oder den jungen Blättchen und den líeimblättern (Kotyledonen) ableitet ( $\mathrm{L}$. H e r m a n n, Müller-Hettlingen). Die Kotyledonen verhalten sich dabei positiv gegen die übrigen Teile.

Es bedarf weiterer Untersuchungen über den Sitz und die Bedeutung dieser Potentialdifferenz. Bemerkenswert ist die Beobachtung, daß, wenn man auf die abgeleitete Wurzelstelle einen Tropfen Wasser bringt, eine starke negative Schwankung bis zu 0,04 Daniell eintritt (Müller-Hettlingen). Dies scheint darauf hinzudeuten, daß die elektrischen Potentiale hier init der Wasserresorption in den Wurzeln in Beziehung stehen.

Nan wird gegen eine elektroosmotische Kraft der Zellmembranen einwenden, daß man bisher geglaubt hat, in den bekannten Beobachtungen über Plasmolyse der Zellen nur mit dern gewöhnlichen osmotischen Druck derselben zu tun zu liaben, und daß bei beginnender Plasmolyse der osmotische Druck der angewendeten Außenflüssigkeit gleich dem der Zellflüssigkeit sei. Nach unserer Theorie kommt aber zu dem letzteren noch die Kraft des Membranpotentials hinzu. Es ist (s. S. 166) $p_{i}+\pi$ $=p_{a}$ im Falle des Gleichgewichts. In den bisherigen Versuchen ist $\theta$ s aber nicht möglich, die Kraft $\pi$ von $\eta_{i}$ zu sondern, und man mißt durch $p_{a}$ die Summe beider. Es bleibt hiernach selbstverständlich auch der Satz bestehen, daß alle Lösungen, welche eben I'lasmolyse bewirken, isosmotisch sind. Es könnte auch der Fall gedacht werden, daß $\pi$ einen negativen Wert annehme, wenn sich unter Einwirkung gewisser Elektrolyte das Membranpotential umkehrte. Man könute ferner gegen die elektroosmotische Membrantheorie den Einwand erheben, daß auch an der Muskelund Nervenfaser bei der Erregung ein Austritt von Wasser stattfinden müßte, während man annimmt, daß, bei der Kontraktion die Muskelfaser ihr Volumen konstant hält. Indessen die schnellen Potentialschwankungen an den quergestreiften Muskeln und den Nerven können einen merkbaren Wasserwechsel nicht herbeiführen, da sie zu kurze Zeit andauern, bevor noch eine merkliche Wasserbewegung eintreteu könnte.

Daß bei der Kontraktion durch Oxydation Wasser gebildet wird, welches zur Abscheidung gelangen müßte, unterliegt wohl keinem Zweifel. Die Verminderung des Membranpotentials durch vorangegangene Reizung und Ermüdung würde hierfür günstig 


\section{- 181}

sein. An sehr langsam reagierenden glatten Muskelfasern könnte auch schon im Verlauf der Potentialschwankung und während der Kontraktion eine Wasserausscheidung möglicherweise beginnen.

\section{Zehntes Kapitel.}

\section{Die Elektrokinese.}

Verhalten der Kolloide und Zellen im elektrischen Potentialgefälle. Die Bewegung der Kernfiden bei der Zellteilung (Karyokinese) als Elektrokinese.

Wir haben schon oben die Erscheinung der Elektrokinese erwähnt (S. 15S), welche dariu besteht, daß kleine in einer Flüssigrkeit suspendierte Partikelchen durch den Strom in der Richtung nach der Anode oder der Kathode hin bewegt werden. Diese Vorgänge sind physiologisch deshalb von besonderem Interesee, weil man sie nicht nur an toten Partikelchen, wie Kohle, Kaolin, Schwefel, Mastix, Stärkekörnchen usw., ferner an kolloidalen Suspensiouen von Metallen und Metalloxyden, sondern auch an organischen Kolloidlösungen und schließlich an lebenden Zellen, Bakterien, einzelligen Organismen und Zellen höherer Organismen beobachtet hat. Endlich findet man auch an mehizelligen niederen Organismen und Larven von Amphibien und jungen Fischen ein $\theta$ durch den galvanischen Strom hervorgerufene Richtung der Bewegungen vor, welche man Galvanotropismus oder Galvanotaxis genannt hat.

Die Ursache der Bewegung aller jener Körperchen in einem Potentialgefälle ist auf ihre elektrische Ladung gegen die Flüssigkeit zurückzufuhren, wie schon oben gedeutet. Diese elektrische Ladung kaun man bei fester Phase der Partikelchen als Folge eines Adsorptionspotentials auffassen, oder, da es ja auch feste Lösungen gibt, als die Folge eines Diffusionspotentials. Die letztere Anschauung erscheint namentlich bei solchen suspendierten Teilchen, welche mehr oder weniger Wassermoleküle binden, wie den Teilchen der Kolloide, sehr plausibel. Bei den Zellen dürfen wir, dieser 
Anschauung folgend, das osmotische Membranpotential, mit dem wir bisher operiert haben, auch konsequenterweise als die Ursache ihrer elektrischen Ladung ansehen.

Nach der Theorie von Helmholtz läßt sich in der Formel von Perrin (S.159) die Geschwindigkeit $u$, mit der sich die Teilchen bewegen, berechnen:

$$
u=\frac{\varepsilon \cdot H \cdot D}{4 \pi \cdot \eta},
$$

worin $\varepsilon$ die Ladung oder das Potential der Teilchen, $H$ das Potentialgefälle des zugeleiteten Stromes, $D$ die Dielektrizitätskonstante und $\eta$ die innere Reibung der Flüssigkeit ist. Es kommt, wie man hieraus erkennt und worauf wir schon bei der Elektroosmose hingewiesen haben, wesentlich auf die Stärke des Potentialgefälles $H$ an, so dab auch bei kleinen Werten von $\varepsilon$ beträchtliche Wirkungen dadurch erzielt werden können.

Die Kiolloide kann man in die Suspensionskolloide und in die hydrophilen Kolloide einteilen ${ }^{1}$ ).

Anorganische Suspensionskolloide sind z. B. die durch gewisse chemische Reaktionen dargestellten Hydroxyde und Sulfide von Schwermetallen, wie $\mathrm{Fe}(\mathrm{OH})_{3}, \mathrm{Al}(\mathrm{OH})_{3}, \mathrm{As}_{2} \mathrm{~S}_{3}, \mathrm{Sb}_{2} \mathrm{~S}_{3}$ usw., deren Teilchen als "disperse Phase" in dem Wasser als "Dispersionsmittel" schweben. Sie verhalten sich alle ähnlich den Kohlesuspensionen oder den feinen Suspensionen von edlen Metallen, Gold, Silber, Platin, welche man durch Zerstänbung von Elektroden dieser Metalle in Flüssigkeiten durch elektrische Schläge als kolloidale Metallösungen herstellt (Bredig).

Die hydrophilen Kolloide sind die eigentlichen Kolloide, von denen die Benennung Kolloid, von Colla (Leim), hergenommen ist. Es sind dies haupsächlich die organischen Kolloide: Eiweißkörper, Leimsubstanzen, Gummi, Stärke, Lecithine usw., wichtige Bestandteile des Protoplasmas. Aber anch unorganische Kolloide dieser Art gibt es, wie die Lösnngen der Kieselsänre. Ihre Teilchen. welche in der Flüssigkeit schweben, sind nicht als feste Phase anzusehen, sondern sie enthalten selbst das Dispersionsmittel, das

1) Siehe hierüber bei Höber, Physikal. Chem. d. Zelle u. Gewebe, 8. 324 . 
Wasser. Man kann sie als komplexe Moleküle betrachten, welche Wassermoleküle binden.

In einem Potentialgefälle bewegen sich nun die Teilchen der Suspensionskolloide wie der hydrophilen Kolloide je nach ihrer Ladung nach der einen oder anderen Elektrode. Ihre Wanderungsgeschwindigkeit ${ }^{1}$ ) in einem Gefälle von 1 Volt auf $1 \mathrm{~cm}$ schwankt zwischen 10 bis $40 \cdot 10^{-5} \mathrm{~cm} / \mathrm{sec}^{-1}$ und ist daher von derselben Größenordnung wie die der meisten Ionen $\left(\mathrm{Na}^{+}=46 \cdot 10^{-5}\right.$, $\mathrm{Cl}^{-}=68 \cdot 10^{-5}$ ). Die Richtung der Bewegung ist von der chemischen Natur des Kolloids abhängig. Die kolloiden Metallhydroxyde von Eisen, Silber, Aluminium, Chrom usw. wandern zur Kathode, ebenso auch kolloide basische Farbstoffe. Dagegen wandern Säuren, wie Kieselsäure, Zinnsäure, Gerbsäure, Mastix, auch Säurefarbstoffe, zur Anode. Die basischen Stoffe sind also positiv, die sauren dagegen negativ geladen. Die positive Ladung der Basen kann man daraus ableiten, daß aus ihnen ihre negativen $\mathrm{HO}^{-}$-Ionen in das Dispersionsmittel hinein dissoziieren, die negative Ladung der Säuren dagegen daraus, daß dies mit ihren positiven $\mathrm{H}^{+}$-Ionen geschieht. Auch ist es wahrscheinlich, daß von ihrer Darstellung her bei den Basen geringe Reste von Alkalien, bei den Säurekolloiden geringe Reste von angewendeten Säuren den Partikelchen anhaften, welche diese Ladungen verstärken.

Wenn man nun zu den Suspensionskolloiden gewisse Elektrolyte hinzusetzt, welche ihre Ladungen vermindern und schließlich umkehren, so vermindert sich zuerst auch die Wanderungsgeschwindigkeit der Partikelchen, es tritt dann ein "isoelektrischer" Zustand ein, bei dem die Geschwindigkeit Null wird, und bei weiterem Zusatz kehrt sich die Bewegung um. In dem isoelektrischen Punkte treten dann Ausfällungen, Flockungen der Kolloide anf. Auf dieser Tatsache beruht die elektrische Theorie der Gerinnung und Ausflockung der Kolloide von Hardy. Alle diese Erscheinungen nimmt man auch an den hydrophilen Kolloiden, z. B. den Eiweißlösungen, wahr. Dies ist von Michaelis und Rona an dem denaturierten Eiweiß (durch Dialyse salzfrei dargestelltes Eiweiß) durch Zusatz sehx verdünnter Säure genauer gemessen worden. Bei einer Konzentration von $\left[\mathrm{H}^{+}\right]=0,3 \cdot 10^{-5}$ ( $\mathrm{g}$ Ion in 1 Liter) wird dieses Eiweiß isoelektrisch und fällt aus.

1) Siehe Höber, l. c., S. 326. 
Man kann daher den Zustand der Suspension in einem Dispersionsmittel, den man auch kurz "Sol" genannt hat, sich daraus erklären, daß die Partikelchen sich vermöge ihrer gleichen elektrischen Ladungen gegenseitig abstoßen und dadurch schwebend erhalten (Bredig). Sobald sie aber in dem isoelektrischen Punkt neutral geworden sind, ballen sie sich zu größeren Aggregaten, Flocken und Fäden zusammen, indem die Adhäsionskräfte in Wirkung treten, und schließlich können sie durch diese Aggregierung ein zusammenhängendes Netzwerk von Fäden bilden, welches in den Lücken die Flüssigkeit einschließt. Diesen letzteren Vorgang nent man Gelatinierung und Gerinnung, den man am ausgesprochensten bei den Leimlösungen, Albuminlösungen und am Blute beobachtet. Die geronnene Masse hat man "Gel" genannt. An den Suspensionen beobachtet man bekanntlich die Browusche Molekularbewegung, welche man aus den Wärmeschwingungen der Flüssigkeitsteilchen erk]ärt hat. Mit Hilfe des Ultramikroskops kann man die kleinsten Teilchen, Mikronen und Submikronen, und ihre Bewegungen beobachten. Werden sie bei der beginnenden Fällung größer, so hören diese Bewegungen auf.

Auch durch Zusatz von Salzen werden häufig Kolloide aus ibren Lösungen ausgefällt. Dieser Vorgang erklärt sich ebenfalls aus der Neutralisation ihrer elektrischen Ladungen. Die Ionen der Salze diffundieren mit verschiedener Geschwindigkeit in die Kolloidpartikelchen hinein oder werden in verschiedener Menge von ihnen gelöst (verschiedener Teilungskoeffizient der Ionen oder verschiedener Adsorptionskoeffizient derselben).

Werden nun die positiven Kolloide von den Inionen, die negativen Kolloide von den Kationen der zugesetzten Elektrolyte neutralisiert, so tritt Ausflockung ein. Diese Voraussetzung bestätigt sich dadurch, daß ceteris paribus die Fällungskraft der wirksamen Ionen von ihrer chemischen Wertigkeit abhängt, d. h. von der Anzahl der elektrischen Einheiten, mit denen sie geladen sind (Hardy). Von Freundlich ist ferner nachgewiesen worden, daß neben der Wertigkeit der Ionen die Adsorbierbarkeit derselben einen positiven Einfluß auf die Fällungskraft ausübt. Darans erklärt es sich, daß unter den einwertigen Ionen besonders die $\mathrm{H}^{+}, \mathrm{Ag}^{+-}$ und $\mathrm{OH}^{-}$-Ionen sich durch Fällungskraft auszeichnen, da sie eine große Adsorptionskonstante besitzen. Gut adsorbierbare Ionen können schon bei geringer Konzentration den isoelektrischen Zu- 
stand und damit Ausfällung der Kolloide berbeiführen. Daher findet man bei gewissen organischen Ionen, z. B. denen des Anilinchlorids, Strychnin-, Morphinchlorids und der Fuchsinverbindungen, starkes Fällungsvermögen vor.

An den hydrophilen Kolloiden, z. B. den Lösungen der Eiweißkörper, beobachtet man ganz ähnliche Erscheinungen wie an den Suspensionskolloiden. Die suspendierten Teilchen muß man sich bei ihnen aber als Moleküle von großem Volumen und Gewicht, mit Wassermolekülen vereinigt vorstellen. Die Aufnahme von Ionen der Elektrolyte geschieht in diesen wahrscheinlich weniger durch Adsorption als vielmehr durch Osmose und Lösung nach gewissen Teilungsverhältnissen. Man konstatiert an den Eiweißlösungen ebenfalls eine Wanderung der Teilchen nach den Elektroden im Potentialgefälle, und zwar wandern dieselben in einer sauren Lösung zur Kathode, in einer alkalischen Lösung zur Anode. In der ersteren nehmen die Eiweißmoleküle einen elektropositiven, in der letzteren einen negativen Charakter an. Man denkt sich, daß in der sauren Lösung die $\mathrm{H}^{+}$-Ionen schneller in das Kolloidmolekül eindringen und sich unter größerem Teilungskoeffizienten darin lösen, als die zugehörigen Anionen, und dadurch demselben positive Spannung verleihen, und da.j in der alkalischen Lösung die $\mathrm{OH}^{-}$-Ionen diese Rolle spielen und den Molekülen negative Spaunung geben. In ganz neutralen Eiweißlösungen findet keine merkliche Wanderung der Moleküle statt, auch nicht bei Zusatz von Neutralsalzen der Alkalien (Pauli). Die Ionen derselben werden uicht in merklich verschiedener Menge von den Eiweißmolekülen aufgenommen. Dadurch unterscheiden sich die hydıophilen Kolloide von den Suspensionskolloiden.

Von Bedeutung für die Theorie der Kolloide ist auch die Tatsache, daß der Zusatz von Nichtelektrolyten, wie Alkoholen, Äther, Zucker, Glyzerin usw., keinen Einflu@ auf die Elektrokinese und die Fällbarkeit der Kolloide ausübt (abgesehen davon, daß Alkohole die Eiweißkörper überhaupt nicht lösen).

Wir können hier auf die vielen Einzelheiten der physikalischen Chemie der Kolloide nicht näher eingehen und verweisen auf die schon oben genannten Werke von Höber, Physikalische Chemie der Zelle und der Gewebe, und auf Fleundlich, Kapillarchemie, sowie auf P. Rona, Allgemeine Chemie der Eiweißkörper (Handb. d. Biochemie von Oppenheimer 1908). 
Von besonderem Interesse für den bier zu behandelnden Gegenstand ist indessen das Verhalten lebender Zellen in Suspensionsmitteln. Solche Beobachtungen hat man an sogenannten Zellsuspensionen angestellt, zu deren Herstellung man namentlich Bakterien und Blutzellen verwendet hat. Diese Zellen wandern unter normalen Bedingungen meist zur Anode, d.h. sie besitzen in der Suspensionsflüssigkeit, die gewöhnlich aus physiologischer ClNa-Lösung besteht, eine negative Ladung. Dies ist durch Versuche an verschiedenen Bakterien (Bechhold u. a.), an Blutkörperchen und Hefezellen (Höber) und auch an Spermatozoen (Lillie) gezeigt worden.

Dieses Verhalten erklärt sich nun befriedigend aus der Membrantheorie, die wir ja auf alle lebenden Zellen zu übertragen haben. Von den in ihnen enthaltenen Elektrolyten wird das Kation in stärkerem Grade nach außen abdissoziiert als das Anion, welches die Zelle negativ ladet. Höber hat gefunden, daß die gewöhnlich negativ geladenen Blutkörperchen durch kleine Mengen in Verbindungen zugesetzter $\mathrm{H}^{+}, \mathrm{Ag}^{+}, \mathrm{Cu}^{++}$oder $\mathrm{F}_{\theta}{ }^{++}+$-Ionen umgeladen werden können und nun zur Kathode wandern. Ebenso kann man die Zellsuspensionen ausfällen durch Zusatz von Elektrolyten, durch welche die Zellen isoelektrisch gemacht werden. Die Zellen senken sich und ballen sich zusammen, ein Vorgang, den man Agglutinierung genannt hat. So kann Bacterium coli (Darmbakterie) durch bestimmte Konzentration von Eisenchlorid ausgefällt werden. Aber auch positive Suspensionskolloide, wie $\mathrm{Fe}\left(\mathrm{OH}_{3}\right), \mathrm{Cr}(\mathrm{OH})_{3}$, können, in Suspensionen zugesetzt, eine Sedimentierung der Bakterien bewirken.

Auch an anderen einzelligen Organismen, den Protozoen, hat man galvanotropische Bewegungen beobachtet, z. B. an den Infusorien. Da aber diese mit Geißeln und Flimmerhaaren versehen sind und außerdem differenzierte Teile, wie MIndspalte, Afterspalte usw., besitzen, so erscheint die Wirkung des galvanischen Stromes schon nicht mehr in ihrer ganzen Peripherie als gleichartig nach allen Richtungen hin. Nichtsdestoweniger bewegen sie sich in dem elektrischen Felde nach dem eiven oder anderen Pole hin. Besonders an Paramäcien, welche an ihrer ganzen Oberfläche mit Flimmerhaaren bedeckt sind, hat man solche Beobachtungen gemacht. Coehn und Barrat (1905) erklären diese Bewegungen durch Ladungen, welche diese Zellen gegen die umgebende 
Flüssigkeit dadurch annehmen, daß ihre Hülle für Anionen (Cl) durchlässiger sei als für Kationen (Na). In verdüunten Salzlösungen oder Wasser nelımen sie daher positive Ladung an und wandern zur Kathode, in Lösungen dagegen, welche konzentrierter sind als die tierischen Flüssigkeiten, laden sie sich durch Aufnahme von Anionen negativ und wandern daher, wie der Versuch zeigt, zur Anode. Bei einer Grenzkonzentration zwischen 0,01 und $0,1 \mathrm{n}-\mathrm{ClNa}$ - oder Bicarbonatlösung verhalten sie sich neutral. In Zuckerlösungen von gleichem osmotischen Druck wie die Salzlösungen wandern sie dagegen immer zur Kathode. Bancroft (1905/06) hingegen sucht die galvanotropischen Bewegungen dieser Organismen auf Reizungen der Flimmerhaare zurückzuführen, indem er gemäß dem Pflügerschen Gesetz von der polaren Erregung eine stärkere Reizung an der Kathode annimmt. Vielleicht kommen bei diesen Zellen beide Ursachen in Betracht.

Noch komplizierter gestalten sich jedenfalls die galvanotropischen Bewegungen an Hetazoen, den mehrzelligen Organismen, besonders denen von höherer Entwickelungsform. Hier haben wir es mit Wirkungen auf die Nervenenden der Haut und bei den Wirbeltier'en auch auf die Gleichgewichtsorgane im Ohrlabyrinth zu tun, wodurch komplizierte Reflexaktionen ausgelöst werden. L. Hermann beobachtete, daß Froschlarven sich im galvanischen Strome mit dem Kopfende nach der Anode einstellen. Dies geschieht auch nach der Köpfung durch Einwirkung des Stromes auf das Rückenmark. Ähnlich verbalten sich Fischembryonen.

Unter den Entwickelungsprozesselı ist es der Yorgang der Zell- und Kernteilung, welcher bekanntlich die Grundlage aller Formbildung bei den Organismen ist, den wir hier in den Kreis unser'er Betrachtungen einbeziehen wollen. Man hat schon seit längerer Zeit vermutet, daß bei diesem elektrische Kräfte eine Rolle spielen; doch sind bis jetzt nur unbestimmte und vage Anschauungen hierüber ausgesprochen worden. Bei dem gegenwärtigen Stande der elektrischen Theorien über die Kolloide, die Zellplasmamembranen und nach der von uns durchgefübrten elektroosmotischen Membrantheorie lassen sich nun Gesichtspunkte gewinnen, welche, wie mir scheint, zu einer befriedigenden Theorio der Kernfädenbewegung, der sogenannten K a ryokinese, führen künnen. Wir wollen im folgenden eine solche Theorie entwickeln, nach welcher die Karyokinese als eine Elektrokinese aufzufassen ist. 
Man ist wohl von allgemein entwickelungsmechanischem Staudpunkt aus berechtigt anzunehmen, daß die Vorgänge der Zellteilung, welche in so übereinstimmeuder typischer Weise bei jeder Entwickeluug von den niedrigsten bis zu den höclisten Organismen wiederkehren, noch auf verhältnismäßig einfachen Kiomponenten physiko-chemischer Natur beruhen. Je komplizierter indes die Organismen sich ausbilden, um so mehr liomponenten dieser Art treten in den Molekularkomplex der lebenden Zellsubstanzen ein, so daß ihre Zergliederung inmer schwieriger wird. Für die Karyokinese hat man nun deshalb die Mitwirkung elektrischer oder magnetischer liräfte vermutet, weil dabei im Innereu

A

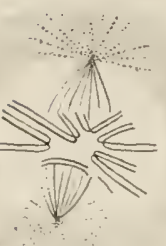

$B \quad$ Fig. 58.

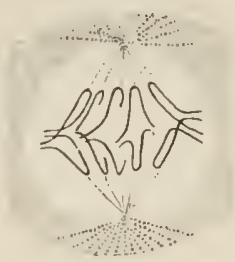

C

D

Schema der Kernteilung. A Kernspindel mit Äquatorialplatte; $B, C, D$ Teilung der Kernschleifen und Bewegung derselben nach den beiden in den Polen der Kernspindel befindlichen Centrosomen.

des Protoplasmas gewisse Strahlungsfiguren anftreten, welche lebhaft an bekannte elektrische uud mignetische Kraftlinien eriunern. Es seien zur Orientierung über die zu betrachtenden Vorgänge der Kernteilung der Eizelle dio wesentlichen Erscheinungen derselben hier kurz angegeben und durch einige Figuren erläutert. Bei der Befruchtung dringt das Spermatozoou in die Eizelle ein, der Kopf, als Kern desselben, vereinigt sich mit dem Eikern, der Faden desselben löst sich auf, während sich aus dem Mittelstück ein kleines Körperchen, das Centrosoma, formt, welches dem neuen Eikern anliegt. Dieses teilt sich nun in zwei Controsomen, welche auseinanderrücken und die Pole einer Strahlıngsfigur bilden, die man die Kernspindel genannt hat. In den Fig. 58 sieht man diese Strahlungsfigur während der einzelnen Stadien der Kernteilung in der Mitte der Eimasse gezeichnet. Zwischen den Polen der Kernspindel liegt der Eikern. Dieser besteht anfangs aus einem ungeordneten linäuel von Fäden, den Kernfäden 
oder. Chromatosomen, welche sich mit Farbstoffen stark färben. Dieselben ordnen sich nach der Vereinigung des Ei- und Spermakernes in einzelnen gleich langen Stücken in der Äquatorialplatte, Fig. 58 A, an, und jeder Kernfaden spaltet sich der Länge nach in zwei Fäden. Nun beginnt die Wanderung derselben, indem die eine Hälfte auf den Kurven der Kernspindel nach dem einen, die andere Hälfte nach dem anderen Centrosoma in Gestalt einer baarnadelförmigen Schleife hinrückt, wie es die Fig. $58 \mathrm{~A}$ bis D angeben. Jeder ursprüngliche Kernfaden zerfällt in zwei, von denen einer nach der einen, der andere nach der anderen Richtung fortbewegt wird. Sind diese Kiernfäden an den Polen der Kernspindel angelangt, so ordnen sie sich dort um das Centrosoma derselben sternförmig an und vereinigen sich hier knäuelartig zu dem neuen Keru der beiden Tochterzellen, während das Protoplasma der Eizelle sich ebenfalls in zwei Hälften teilt.

Die Kernfäden, welche sich in dem Eikern befinden und geteilt haben, sind nun nach unserer Ansicht Gebilde, welche innerbalb des Plasmas, in dem sie liegen, ebenfalls elektrische Spanriung annehmen. Man darf dieselben wohl als organisierte Gebilde komplizierter Struktur, vergleichbar den Fibrillen der Muskel- und Nervenfaser und zusammengesetzt aus mannigfachen Kolloiden im Gelzustande, ansehen. Man darf ferner voraussetzen, daß Kat- und Anionen der Elektrolyte des Plasmas von ihnen in verschiedenem Grade aufgenommen werden, sei es durch Osmose oder Adsorption, oder daß in ihrem Inneren befiudliche Ionen in verschiedenem Grade nach außen in das Plasma abdissoziiert werden. Infolgedessen müssen sie eine elektrische Spannung gegen das umgebende Plasma erhalten, ähnlich wie es bei der Nerven- und Muskelfaser, bei Zellen in der Ernährungsflüssigkeit oder bei den Kolloidpartikelchen in einer Flüssigkeit der Fall ist. Nach der Membrantheorie dringt das positive Ion eines Elektrolyten des Plasmas schueller, das negative dagegen sehr viel langsamer durch die semipermeable Membran der Zelle nach außen. Erteilen wir auch den Kernfäden der Eizelle eine solche semipermeable Membran von ähnlicher Beschaffenheit und nehmen auch an, daß das negative Ion des Plasmaelektrolyten schwer oder gar nicht, dagegen das positive Ion desselben leicht eindringen kann, so würden die Kernfäden gegen das Plasma positive Spannung annehmen. Es steht natürlich auch der Voraussetzung nichts im Wege, daß die Kern- 
fäden einen besonderen Elektrolyten enthalten, dessen negatives Ion mit viel größerer Geschwindigkeit nach außen diffundiert als das positive. Derselbe könnte seiner chemischen Natur nach zu den Basen gehören, da deren $\mathrm{OH}^{-}$-Ion die größere Wanderungsgeschwindigkeit besitzt, während wir für das Zellplasma als wirksamen Elektrolyten eine Säure oder ein saures Salz (z. B. $\mathrm{K}_{2} \mathrm{HPO}_{4}$ ) vermutet haben.

Es kann nun ferner wohl als sicher angenommen werden, daß wir den eindringenden Spermakern und ganz besonders das sich vom Spermatozoon absondernde Centrosoma bei der Befruchtung and Zellteilung als ein Zentrum lebhafter chemischer $A$ ktion betrachten können. Es liegt nicht fern, dieses nach unseren heutigen Kenntnissen als ein gewisse Fermente enthaltendes Körperchen besonderer Art anzusehen, welches geeignet ist, in dem umgebenden Protoplasma Spaltungs- und Oxydationsprozesse zu veranlassen. Durch die Vereinigung des Ei- and Spermakernes werden dagegen, wie es scheint, Prozesse mehr entgegengesetzter Natur, d. h. Assimilations- und Wachstumsprozesse angeregt, welche ebenfalls durch gewisse Fermente anderer Art im Spermakern hervorgerufen sein mögen und sich durch die Spaltung der Kernfäden kundgeben.

Das Auseinanderrücken der beiden durch Teilung entstandenen Centrosomen mag schon ein Vorgang elektrokinetischer Natur sein. Wir denken uns zu diesem Ende, daß sich die beiden Centrosomen wie zwei Kolloidkörperchen gleicher elektrischer Ladung verhalten und sich gegenseitig abstoßen. Eine solche Ladung kann dadurch entstehen, daß sie Ionen der Elektrolyte des Plasmas in verschiedenem Grade lösen oder adsorbieren. Nachdem sie nun ihre Lage $\mathrm{zu}$ beiden Seiten des Eikernes eingenommen haben, bilden sio jederseits in dem Plasma ein Zentrum eines osmotischen Druckgefälles, in welchem sich nach allen Richtungen hin die Moleküle und Ionen der entstehenden Spaltungs - und Oxydationsprodukte bewegen. Zu diesen können wir die Aminosäuren, welche aus der Spaltung der Eiweiße hervorgeben, ferner die Fettsäuren aus der Spaltung der Fette und Kohlehydrate und endlich die entstehende Kohlensäure rechnen. Das Druckgefälle muß sich so gestalten, wie es die entstehenden Strahlungsfiguren angeben. Zwei Maxima desselben liegen in den beiden Centrosomen, zwischen ihnen liegt ein Ninimum in dem die Ïquatorialplatte bildenden Eikern, und nach der Peripherie der Eizelle hin fällt der osmotische Druck 
nach allen Richtungen hin ebenfalls ab. In einem solchen osmotischen Druckgefälle von Kö̈rpern, welche als Säureı mehr oder weniger stark elektrolytisch dissoziiert sind, entsteht nun bekanntlich wie in einem Diffusionszylinder eines Elektrolyten (s. S. 33, Fig. 10) ein elektrisches Potentialgefälle, welches ganz denselben Kurvenverlauf besitzt wie das osmotische. Betrachten wir die entstehenden Säuren als die wirksamen Elektrolyte, so wandern ihre $\mathrm{H}^{+}$-Kationen schneller als ihre Anionen, und die Centrosomen nehmen ein Maximum negativer Spannung an, welches nach der Äquatorialplatte und nach der Peripherio in Gestalt der Strahlungsfigur abfällt. Man kann daher in der üblichen Bezeichnung der Potentiallehre die Äquatorialplatte des Eikernes und die Zellperipherie als Senken und die beiden Centrosomen als Quellen eines negativ elektrischen Potentialfeldes bezeichnen.

Sind nun die elektrischen Ladungen zwischen Centrosomen, Plasma und Kernfäden in der angenommenen Weise verteilt, so muß eine elektrokinetische Bewegung der letzteren in der Weise stattinden, dafj sie, wie es der Fall ist, auf den Kurven der Kernspindel, welche das osmotische und elektrische Potentialgefälle angeben, von der Äquatorialplatte nach den Centrosomen hin vorwärts rücken. Es ist in dem Ei nicht etwa ein Leitungsstrom als Ursache dieser Bewegung vorhanden, sondern es entsteht in demselben ein Konvektionsstrom, indem die Kernfäden positive Elektrizität von der $\ddot{A} q u a t o r i a l p l a t t e n a c h$ den Centrosomen transportieren.

Fig. 59 soll ein Bild dieser Bewegung infolge der Verteilung der elektrischen Spannungen geben. In den beiden Centrosomen herrsche das Maximum des osmotischen Drackes und der Elektrolytkonzentration $C$, in der Äquatorialplatte dagegen sei dieser Wert $c$. Nach $C C$ ist daher der negative und nach $c c c$ der positive Pol des entstehenden Konvektionstromes zu verlegen, wie die Zeichen angeben. Die Kernfäden, in der Mitte ihres Weges befindlich, dargestellt, werden vermöge ihrer positiven Ladung nach $C C$ hingetrieben. Sie geben die negative Ladung der betreffenden Anionen an die positive Elektrizität der Äquatorialplatte ab und leiten ihre positive Elektrizität der negativen der Centrosomen zu.

Wir können noch folgendes zugunsten dieser Theorie anführen. Die schleifen- oder haarnadelförmige Gestalt der Kern- 
fäden und das Vorangehen der Umbiegestelle bei der Bewegung ist, soviel ich weiß, bis jetzt noch keiner mechanischen Betrachtung unterzogen worden. Aus dieser Tatsache geht aber klar hervor, daß die bewegende Kralt in ihrer Mitte am größten sein muß und nach den beiden Enden hin abnimmt.

Bewegen wir einen biegsamen Faden in einer Flüssigkeit, indem wir ihn in seiner Mitte mit einem Stabe vorwärts führen, so nimmt er bekanntlich infolge der Reibung eine solche Schleifenform an.

Die Kraft der elektrokinetischen Bewegung ist nun aber nach Formel (19) (S. 159) ebenfalls wie die Geschwindigkeit proportional der Ladung $\varepsilon$, da die Beschleunigung in jedem Moment

kig. 59.

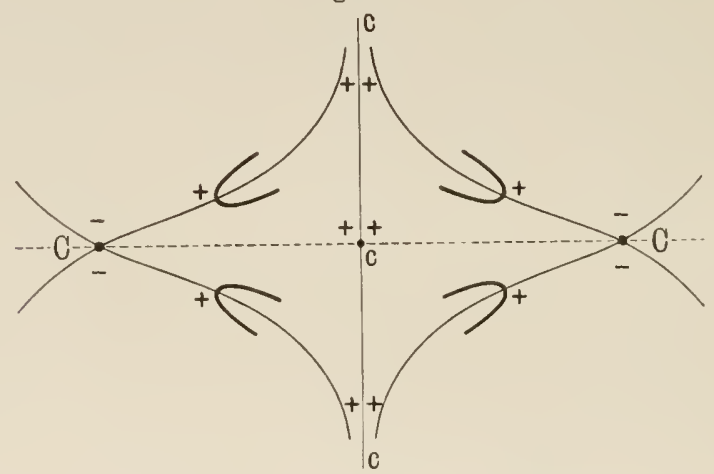

Elektrokinese der Kerufäden im Potentialgefälle.

durch die Reibung aufgehoben wird. Diese Ladung wird aber in den Kernfäden ein Maximum in ihrer Mitte haben, äbnlich wie an Nerven- und Muskelfasern die stärkste positive Spannung in ihrer Nitte (Äquator s. S. 6) herrscht; denn wir können ihre Membran und Substanz an den natürlichen Querschnittsenden in stärkerer Veränderung begriffen ansehen, wodurch die Größe $\varepsilon$ von der Mitte nach den Enden hin abnimmt. Diese Unterschiede brauchen nur sehr gering zu sein, wie wir sie an den unverletzten Muskeln gewöhnlich vorfinden. (s. S. 9) ${ }^{\mathrm{I}}$ ).

1) Um der aufgestellten Theorie eine experimentelle Stütze zu geben, habe ich den Plan, feine kurze Nervenfäden in einer geeigneten Flüssigkeit (Serum, Eiweißkochsalzlösung) zu suspendieren und in ein starkes Potentialgefälle zu bringen. Nach der Membrantheorie wäre 
Dieser Prozeßder Kernteilung wiederholt sich bei den weiteren Zellteilnngen während der Entwickelung sowohl als auch beim Wachsen der Gewebe in analoger Weise. Es sei noch bemerkt, da sich auch schon beim Einrücken des Spermakermes und nach Vereinigung desselben mit dem Eikern von diesen aus eine radiäre Strahlung entwickelt, welche bis an die Peripherie der Eizelle hinreicht.

Es soll nun hier keineswegs eine vollständige Theorie der Kern- und Zellteilung gegeben werden. Es sollen vielmehr gewisse Vorbedingungen für den Vorgang der Karyokinese, d.h. der Bewegung der durch Teilung gebildeten Kiernfäden nach den beiden Centrosomen, als zunächst unerklärte Zellprozesse vorausgesetzt werden, und es soll nur versucht werden, diesen Bewegungsvorgang als eine Elektrokinese zu denten.

Auch schon in der Äquatorialplatte stellen sich die Kernfäden (s. Fig. $58 \mathrm{~A}$ ), in Schleifenform geordnet, in der Äquatorialebene ein. Das ist darauf zurückzuführen, daß auch von der Äquatorialplatte aus Potentiallinien nach der Peripherie der Zelle hinziehen, welche in der Äquatorialebene das stärkste Gefälle haben. Daher ordnen sich die Kernfäden in dieser Ehene nach dem Mittelpunkt derselben gerichtet an, und nachdem sie sich geteilt und durch gleichartige Ladung parweise gegenseitig abgestoßen haben, wenden sie sich den nach den Centrosomen hin gerichteten Potentiallinien zu.

Ebenso haben wir die in dem Protoplasma des Eies und in der Kiernspindel auftretende Strahlung, welche aus Reihen von Körnchen besteht and uns den Verlauf der Potentialkurven sichtbar angibt, als eine Folge des Potentialgefälles anzusehen. Diese Körnchen, aus kleine:en oder größeren Aggregaten von Kolloidpartikelchen bestehend, werden ebenfalls eine elektrische Spannung gegen die Plasmaflüssigkeit annehmen, und wenn sie auch bei zu geringer Ladung and erheblicher Reibung niclit merklich bewegt werden, so erhalten sie doch infolge dieser Wirkung eine gerichtete Anordnung in den Potentialkurven des osmotischen und elektrischen Druckgefälles. Vielleicht bewegen sich die einen von ihnen nach der Anode, die anderen nach der Kathode, wodurch ein scheinbarer Stillstand eintritt.

zu erwarten, daß sie sich nach der Anode hin bewegten, vielleicht auch in Schleifenform. Die feinen Nervenästchen der Rückenhaut des Frosches wïrden sich zu diesem Versuch gut eignen.

Bernstein, Elektrobiologie. 
Die Kraft und Geschwindigkeit, mit welcher die Fortführung der Kernfäden erfolgt, ist nach Formel (18) und (19) wesentlich von dem Potentialgefälle $H$ abhängig. Dasselbe muß aber auch in diesem Falle selbst bei verhältnismäßig geringen Werten der Potentialdifferenz zwischen Centrosomen und Äquatorialplatte wegen der geringen Entfernung beider voneinander ein beträchtliches sein. Ebenso braucht die Ladung $\varepsilon$ der Kernfäden keine große zu sein. Beide Größen können innerhalb derjenigen Grenzen liegen, welche wir sonst an Zellen beobachten. Wenn wir daher für diese Potentiale die Werte des Membranpotentials der Muskeln oder Drüsen von 0,08 bis 0,1 Volt aunehmen, und bei einigen Eiern die Entfernung der Äquatorialplatte von den Centrosomen messen, so hätten wir in der Formel

$$
u=\frac{\varepsilon \cdot H \cdot D}{4 \pi \eta}
$$

Zahlenwerte für $H$ und $\varepsilon$ gewonnen, und wenn wir für die Dielektrizitätskonstante $D$ und innere Reibung $\eta$ gewisse Werte als angenäherte einsetzen, so würden wir für $u$ einen Wert erhalten, den wir mit der beobachteten Geschwindigkeit der Kernfäden vergleichen könnten.

Eine genauere Berechnung dieser Art läßt sich vorläufig aus Mangel an Daten über die Entfernung der Centrosomen voneinander im Beginn der Kernteilung und über die Geschwindigkeit der Kernfäden nicht ausfübren. Doch möchte ich nicht unterlassen, aus einigen in der Literatur gefundenen Angaben darüber eine ungefähre Berechnung anzustellen. Für die Zeit von der Befruchtung bis zur. Vereinigung des Spermakernes mit dem Eiker'n am Seeigelei finde ich bei 0 . Hertwig ${ }^{1}$ ) einen Wert von 15 Minuten angegeben, ferner für die Zeit von der Befruchtung bis zum Beginn der ersten Teilung (bzw. bis zum Hantelstadium des Kernes) in zwei Fällen 75 Minuten. Ziehen wir also von letzterer Zeit die 15 Minuten für die Vereinigung der Kerne ab, so wollen wir für die Zeit, in welcher sich die Kernfäden von der Iquatorialplatte nach den beiden Centrosomen bewegen, 60 Minuten annehmen. Für den Abstand der beiden Centrosomen von der Ïquatorialplatte möchte ich nur schätzungsweise - denn ich

$\left.{ }^{1}\right)$ Untersuchungen z. Morphol. u. Physiol. der Zelle 1887, Heft 5, S. 45 u. 83 . 
habe in der Literatur bisher keine Messungen über diese Größe finden können - 0,01 cm ansetzen, und ich glaube, daßs selbst in großen Eiern von Wirbeltieren diese Entfernung über einen ähnlichen Wert nicht wesentlich hinausgehen wird. Nach diesen Annahmen würde die Geschwindigkeit der Kernfäden bei der Karyokinese etwa $3 \cdot 10^{-6} \mathrm{~cm} / \mathrm{sec}^{-1}$ betragen.

Um nun nach der Formel

$$
u=\frac{\varepsilon \cdot H \cdot D}{4 \pi \cdot \eta}
$$

eine Berechnung anstellen zu können, wollen wir für die Ladung $\varepsilon$ der Kernfäden 0,1 Volt annehmen; in mechanischem Maße ausgedrückt wäre demnach

$$
\varepsilon=\frac{1}{3000} \mathrm{~cm}^{1 / 2} \cdot \mathrm{g}^{1 / 2} \cdot \mathrm{sec}^{-1} \text {. }
$$

Das Potentialgefälle $H$ in absolutem Maße wäre hiernach gleich

$$
\frac{1}{3000 \cdot 0,01} \mathrm{~cm}^{-1 / 2} \mathrm{~g}^{1 / 2} \mathrm{sec}^{-1} \text {. }
$$

Für die Dielektrizitätskonstante $D$ des Eiprotoplasmas können wir leider einen Wert nicht angeben; aber es wird erlaubt sein, statt derselben diejenige des Wasser's gleich $80^{1}$ ) anzunehmen.

Setzt man endlich für die Reibungskonstante $\eta$ einen Wert, welcher etwa zehnmal größer genommen werden mag als der, welchen Hürthle für das Blut gleich 5 gefunden hat, so wäre in diesem Fallo

$$
\eta=\check{5} 0 \mathrm{~cm}^{-1} \cdot g \cdot \mathrm{sec}^{-1} \text {. }
$$

Man erbält hiernach:

$$
u=1,5 \cdot 10^{-6} \mathrm{~cm} \cdot \mathrm{sec}^{-1} \text {. }
$$

Die Übereinstimmung des beobachteten und elektrokinetisch berechneten Wertes der Geschwindigkeit der Kernfäden bei der Karyokinese ist der Größenordnung nach eine so befriedigende, daß man in dieser eine gute Stütze der aufgestellten Theorie erblicken darf. Die angenommenen Werte für die Dielektrizitätskonstante und die innere Reibung des Eiplasmas könnten vielleicht als sehr willkürlich erscheinen; sie haben aber auf das Resultat keinen sehr großen Einfluß, denn selbst wenn man sie

$\left.{ }^{1}\right)$ Siehe Landolt u. Börnstein, Tabellen usw. 
zwischen 1 und den oben angenommenen Werten beliebig variieren ließe, so würden die berechneten Geschwindigkeiten doch nur zwischen den Grenzen von $18 \cdot 10^{-7}$ und $7 \cdot 10^{-5} \mathrm{~cm} \cdot \mathrm{sec}^{-1}$ schwanken.

Die wesentliche Bedeutung dieses Resultates besteht eben darin, daß die Geschwindigkeit der Kernfäden der Größenordnung nach den Geschwindigkeiten, welche die Kolloide in einem Potentialgefälle von derselben Größe zeigen w ürden, sehr nahe steht. Letztere Geschwindigkeiten (s. S. 183) hat man in einem Potentialgefälle von 1 Volt pro Zentimeter zu etwa 10 bis $40.10^{-5} \mathrm{~cm} / \mathrm{sec}^{-1}$ gefunden, also würde sie in einem Potentialgefälle von 0,1 Volt pro $0,01 \mathrm{~cm}$, wie wir es in der Eizelle angenommen haben, 1 bis $4.10^{-5} \mathrm{~cm} \cdot \mathrm{sec}^{-1}$ betragen. Daß die gefundenen und berechneten Geschwindigkeiten der Kernfäden etwa um das Zehnfache kleiner (1,5 und $\left.3 \cdot 10^{-6} \mathrm{~cm} / \mathrm{sec}^{-1}\right)$ erscheinen, kann auf Rechnung der starken inneren Reibung im Ei gesetzt werden.

Soweit wäre es gelungen, einen in allen Eizellen von den niedrigsten bis zu den höchsten Organismen der Tier- und Pflanzenwelt in typischer Weise wiederkehrenden Proze $B$ in der Kern- und Zellteilung auf eine physiko-chemische Basis zu stellen. Schon oben ist betont, daß dies freilich nur ein Teil des Gesamtprozesses ist, welchen die Zellteilung darstellt. Dieser Teil bezieht sich nur auf die Bewegung der Kernfäden von dem Orte ihrer Bildung, der Äquatorialplatte, welche aus den vereinigten Ei- und Spermakernen hervorgeht, nach den Centrosomen, die sich in gewisser Entfernung von derselben zu beiden Seiten eingestellt haben, auf dem Wege der Keruspindelstrahlen. Alle übrigen vorausgehenden wie nachfolgenden Vorgänge im Kern wie in der ganzen Eizelle harren weiterer Erklärung in physiko-chemischer Richtung. Doch scheint mir für eine solche Forschung das erhaltene Resultat nicht ohne Bedeutung.

\section{Zusammenfassung und SchluBbetrachtung.}

Werfen wir einen Rückblick auf das gesamte, in den vorangegangenen Kapiteln behandelte Gebiet, so müssen wir nach den gewonnenen Resultaten dem ganzen Stoff eine andere Anordnung geben als diejenige, in welcher wir schrittweise vorgegangen sind. 
Nach ihrer historischen Entwickelung mußte die Untersuchung von den elektrischen Strömen der Muskeln und Nerven ausgehen. Nachdem aber die physiko-chemische Forschung im Gebiete der Elektroosmose und Elektrokinese zu den elektrischen Eigenschaften der Kolloide und denen der in Flüssigkeiten suspendierten Zellen vorgeschritten war, und nachdem sich durch Aufstellung einer Membrantheorie der Zellen alle behandelten Erscheinungen unter gemeinsame Gesichtspunkte zusammenfassen ließen, erscheint es nunmehr gerechtfertigt, a uch in der Darstellung des ganzen Gebietes nach physiko-chemischen Prinzipien und auch nach biologischen Prinzipieu vom Einfacheren zum Komplizierteren vorzugehen.

Stellt man sich auf den Standpunkt einer Entwickelungslehre, welche den Übergang von der leblosen zur lebenden Natur zu ergründen sucht, so wird man geneigt sein, den Ursprung der bioelektrischen Erscheinungen in den elektrischen Eigenschaften der Kolloide zu suchen, die ja einen wesentlichen Bestandteil der lebenden Substanz der Organismen bilden.

Die elektrischen Eigenschaften der Kolloide spielen bekanntlich in dem großen Gebiete der Kolloidchemie eine wesentliche Rolle. Wio im letzten Kapitel berichtet, laden sich bei Gegenwart, von Elektrolyten die liolloidmoleküle uud ihre Komplexe (Submikronen, Mikronen) in ihren Suspensionsflüssigkeiten elektrisch, entweder positiv oder negativ, indem sie entweder positive oder negative Ionen stärker binden, sei es durch Diffusiou oder Adsorption derselben. Infolgedessen wandern die Kolloidmoleküle und -partikelchen in einem Potentialgefalle zur Anode oder Kathode des zugeleiteten Stromes; es tritt eine Elektrokinese auf. Die Fällungen, Ausflockungen und Gelatinierungen von Kolloidlösungen hat man auf die elektrischen Ladungen der Moleküle zurückgeführt, indem man gezeigt hat, daß sie bei diesen Vorgängen elektrisch neutral werden (isoelektrischer Punkt s. S. 183).

Die Erscheinungen der Elektrokinese haben sich nun auch an vielen niederen einzelligen Mikroorganismen nachweisen lassen und ebenso an freien Zellen höherer Organismen (s. S. 186). Man muß daraus schlielen, daß diese Zellen sich ebenfalls in einer Flüssigkeit, in der sie suspendiert sind, elektrisch laden, indem sie die An- und Kationen der in der Flüssigkeit oder in ihrem Inneren enthaltenen Elektrolyte in verschieden starkem Grade durch Diffusion oder Adsorption binden. Es ist daher sehr wahr- 
scheinlich, daß die elektrischen Eigenschaften der Kolloide, aus denen die lebende Substanz der Zellen hauptsächlich zusammengesetzt ist, auch die Ursache dieses merkwürdigen Verhaltens der Zellen im elektrischen Potentialgefälle sind.

Die bekannten Unter'suchungen über den osmotischen Druck haben nun zu der Überzeugung geführt, daß die lebenden Zellen mit einer semipermeabeln Plasmamembran ausgestattet sind, welche den Ein - und Austritt von Molekülen und Ionen verschiedener gelöster Stoffe und des Wassers reguliert. Da diese Plasmamembran im wesentlichen aus kolloiden Körpern zusammengesetzt ist, so ist anzunehmen, daß ihre Eigenschaften auf denen der Kolloide beruhen. Wir kömnen allerdings bis jetzt nicht angeben, welche Konstitution diese Membranen im lebenden Zustande der Zellen haben und wie sie sich von den toten Membranen und den Kolloidkörpern in ihrer Konstitution unterscheiden. Aber die Beobachtung hat ergeben, daß man sie als Diaphragmen ansehen darf, welche gewisse Moleküle und Ionen ron Elektrolyten durchtreten lassen, andere dagegen nicht (s. S. 88).

Die bioelektrischen Erscheinungen können daher auf das Vorhandensein einer Plasmamembran der Zellen zurückgeführt werden, welche für die Anionen und Kationen der Elektrolyte im Inneren der Zellen oder in der Außenflüssigkeit verschiedene Permeabilität, oder verschiedenes Adsorptions- bzw. Lösungsvermögen besitzt. Diese Aunahme bildet die Grundlage der von uns ausführlich dargestellten Membrantheorie (s. 5. Kap.).

Tom genetischen Standpunkte aus darf man sich daher vorstellen, daß die Entstehung elektrischer Eigenschaften mit der Bildung organisierter lebender Substanz aus dem leblosen Material bereits begonnen hat. Inwieweit sich solche Eigenschaften an formloser lebender Masse, wie z. B. an den Plasmodien der Schleimpilze, werden nachweisen lassen, mag der Untersuchung noch vorbebalten bleiben. Die in Zellen geformte lebende Substanz hat diese Eigenschaften zu höherer Entwickelung gefülırt, und so sehen wir, daß mit der Differenzierung der Zellen bei höherer Entwickelung der Organismen die elektrischen Eigenschaften der Zellen und Organe immer deutlicher in ihrer Beziehung zu den Funktionen derselben zum Vorschein kommen. Das elektrische Potential einer Plasmamembran erzeugt daher in Zellen verschiedener Funktion entsprechende Wirkungen. 
Die Grundbedeutung des Membranpotentials der Zellen haben wir nun (s. 9. Kap.) darin gefunden, dabes neben dem osmotischen Druck des Zellinhaltes den Wassergehalt der Zelle reguliert.

Es ist eine fundamentale Eigenschaft der lebenden Gewebe, soweit wir sie bisher haben untersuchen könuen (äußere Haut, Muskel, Pflanzenblätter), also auch ihrer Zellen, daß sie das in der Zellflüssigkeit befindliche Wasser fester binden als im toten Zustande, und diese Eigeuschaft haben wir vermöge der Elektroosmose aus dem Membranpotential der Zellen hergeleitet.

Da man annehmen darf, daß sich die ersten einzelligen Organismen in dem Meerwasser gebildet haben, welches einen hohen osmotischen Druck infolge seines Salzgehaltes besitzt, so muß die Wirkung des Membranpotentials bei diesen von vornherein eine große Bedeutung für die Stoffwechselvorgänge gewonnen haben. Diese Eigenschaft der Zellmembranen hat sich bei der phylogenetischen Entwickelung der Organismen weiter vererbt und mannigfaltig modifiziert. Die Semipermeabilität der Zellmembranen, vermöge deren sie je nach ihrer Konstitution eine gleichsam auswählende Durchlässigkeit und Undurchlässigkeit fül gewisse Moleküle von Nichtelektrolyten, Elektrolyten und deren Ionen erbalten, wird neben dem Membranpotential einen großen Einfluß auf die inneren chemischen Prozesse des Stoffwechsels und den damit verknüpften Energiewechsel ausüben müssen. Daß daher auch bei der Weiterentwickelung der Organismen des Tier- und Pflanzenreiches in dem süßen Wasser der Erdoberfläche und auf dem Lande das ursprünglich im Meerwasser entstandene Membranpotential der Zellen eine wesentliche Rolle im Leben derselben und der Organe spielt, wird einleuchten.

Bei den einfachsten mehrzelligen Organismen, bei denen noch keine Differenzierung der Zellen erfolgt ist, kann auch das Membranpotential keine weitere Bedeutung als die der Wasserregulierung gewonnen haben. Sobald aber eine Differenzierung der Zellen und danit auch eine Arbeitsteilung in ihrer Funktion eingetreten ist, wird damit auch eine Ursache für die Abänderung ihrer elektrischen Beschaffenheit gegeben sein. Bei den einfachsten tierischen Metazoen (mehrzellige Tiere) finden wir bekanntlich in der Gastrulaform eine Scheidung in die zwei Kieimblätter, das Ektoderm und Entoderm, vor, deren Zellen verschiedene Funktion besitzen. Auf 
dieser Entwickelungsstufe sind in dem Entoderm zuerst Sekretionszellen entstanden, welche Sekrete zum Zwecke der Verdauung erzengt haben, und daher darf man sich auch vorstellen, daß bei dieser Differenzierung in bezug auf For'm und Funktion die Plasmamembran sich gewandelt hat. Wir haben oben (8. Kapitel) dio Sekretionszelleu als solche Zellen angesehen, bei denen durch die chemischen Veränderungen im Inneren die Plasmahaut an der äußeren sezernierenden Seite bei der Sekretion zerstört wird. Infolgedessen erzeugen sie eine Potentialdifferenz zwischen der äußeren und inneren Fläche einer sezernierenden Haut (Schleimhaut oder äußerer Haut). Das Membranpotential der inneren Seite der Zellen erzengt nach der oben aufgestellten Theorie eine Wasser treibende Kraft, welche bei der Sekretion zur Wirkung kommt.

Ebenso mögen auch bei der Entwickelung niederster Metazoen Resorptionszellen in dem Entoderm entstanden sein, bei deren Tätigkeit auch das Memblaupotential zur Förderung des Flüssigkeitsstromes in die Gewebe beizutragen begonnen hatte, und die sich bei weiterer höherer Entwickelung der Organisation in die spezifischen Resorptionszellen der Darmschleimhaut und ibrer Zotten umgebildet haben. Für diese Zellen haben wir nach der aufgestellten Theorie das Membranpotential der äußeren resorbierenden Seite derselben als Quelle einer Wasser treibenden Kraft vermutet.

Was wir hier von den Zellen der tierischen Organismen in bezug auf die Bedeutung des Membranpotentials gesagt habeu, können wir auch auf die Zellen der pflanzlichen Organismen übertragen. Die einzelligen Pflanzen, zu denen man die Bakterien rechnet, sind den Protozoen in ihren wesentlichen physiologischen Eigenschaften gleich. An diesen sind ja namentlich die Erscheinungen der Elektrokinese festgestellt worden. Sie besitzen also nach unserer Theorie ein Membranpotential. I)ie physiologische Bedeutung desselben werden wir nach anserer Auffassung auch in der Regulierung des Wassergehaltes zu suchen haben. Die Festhaltung vou Wasser in den Keimen und Sporen von Pilzen auch im lufttrockenen Zustande scheint mir für die Erhaltung der Keimfähigkeit von besonderer Wichtigkeit zu sein. Bei den aus der phylogenetischen Entwickelungsreihe hervorgegangenen mehrzelligen Pflanzen ist das Membranpotential der Zellen bei 
der Differenzierung derselben und ihrer Funktionen zu ähnlicher Bedeutung gelangt wie bei den tierischen Organismen. Es ist mir freilich noch nicht möglich, wach den gegebenen Tatsachen über die Wasserbewegung in den Pflanzen diese Bedeutung im allgemeinen und speziellen an den verschiedenen Pflanzenorganen nachzuweisen. Hierzu wären besondere Untersuchungen über diesen Gegenstand erforderlich. Indessen sind ja bekanntlich die entwickelteren Pflanzen auch mit Drüsen ausgestattet, welche Wasser, Zucker und sogar Verdauungsfermente (s. Dionaea, S. 176) ausscheiden. Das wichtigste Resorptionsorgan der höheren Pflanze, die Wurzel, hat aber die Aufgabe, das Wasser aus dem Boden aufzusaugen und den übrigen Organen zuzutreiben. Es ist bisher noch nicht gelungen, die Vorgänge der Wasserbewegung in der Pflanze aus den Gesetzen des osmotischen Druckes und der Kapillarität allein zu erklären (s. Pfeffer, Pflanzenphysiologie I, Kap. VI), wie man dies eine Zeitlang geglaubt hatte. Da liegt es sehr nahe, zu vermuten, daß es elektrische Potentiale sind, welche sich zu der osmotischen Energie hinzugesellen, um das. Wasser bis in die Spitzen der höchsten Bäume zu treiben. Die Untersuchungen über das Bluten der Pflanzen, das besonders stark im Frühjahr beim Aıschneiden der Stämme uud Äste eintritt, haben ergeben, daß von den Wurzeln ein positiver Druck erzeugt wird, der beim Weinstock eine Höhe von über einer Atmosphäre erreichen kann. Da der ausfließende Saft nur eine geringe Kouzentration besitzt, so geuügt osmotische Energie nicht zur Erklärung dieses Wasserstromes. Es eröffuen sich daher von den oben ausgesprochenen Anschaungen aus neue Gesichtspunkte für weitere Untersuchungen in diesem Gebiete der Pflanzenphysiologie.

Einen ganz speziellen Fall der Wasserbewegung bei den Pflanzen haben wir aber bereits in den Kreis unserer Betrachtung gezogen und aus der Membrantheorie zu erklären gesucht. Das ist der Vorgang, welcher bei den Reizbewegu gen del. Pflanzen stattfindet (s. oben 9. Kap., S. $174 \mathrm{ff}_{2}$ ). Hierbei tritt aus den reizbaren Zellen Wasser in abführende Gefäße aus, wodurch sie sich zusammenziehen und die Bewegung verursachen. Infolge der Abnahme des Membranpotentials der gereizten Zellen geht nach unserer Theorie Wasser des Zellinhaltes durch die Membran nach außen und wird bei der Rückkehr zur 
Rube durch das sich wieder vermehrende Membranpotential in die Zellen zurückbefördert.

Wir haben nun ferner einen Vorgang in den Kreis unserer Betrachtungen gezogen, welcher sich an dem Kern der Zellen abspielt: das ist die Bewegung der Kernfäden bei der Kernteilung, welche der Zellteilung vorausgeht. Daß in entwickelungsmechanischem Sinne der Zellkern als ein aus dem Protoplasma entstandenes Produkt anzusehen ist, kann wohl kaum zweifelhaft sein; denn weun es aucl nicht gelungen ist, in der jetzigen Lebewelt kerulose Zellen nachzuweisen, so ist doch vorauszusetzen, daß formlose lebende Materie bzw. Zellen obne Kerne einst vorhanden gewesen sind. Erst bei der weiteren phylogenetischen Entwickelung hat sich, wie es scheint, das Vermögen der Vererbung in dem entstandenen Kern konzentriert, und so betrachtet man ja allgemein die Kerne der Generationszellen als die Träger der erblichen Eigenschaften der Organismen. Die erblichen Eigenschaften sind, wie man annimmt, hauptsächlich in den aus der Kernmasse sich differenzierenden Kernfäden enthalten, welche bei der Kernteilung sich teilen und in die 'Tochterkerne eintreten. So kompliziert und verschiedenartig daher auch diese Kernfäden zusammengesetzt sein mögen, um so komplizierter je höher entwickelt der aus der Eizelle hervorgehende Organismus ist, der Vorgang der Bewegung der Kernfäden, die Karyokinese, geht von den niedersten bis zu den höchsten Organismen in derselben typischen Weise vor sich. Es ist daher sehr wahrscheinlich, daß er auf einem physiko-chemischen Prozeß einfacherer Natur beruht, welcher von den zuerst entstandenen kernhaltigen Zellen bei der Teilung erworben wurde, sich für die Weiterentwickelung als nützlich erwiesen und sich daher weiter vererbt hat. Um diesen Vorgang zu erklären, haben wir (s. 10. Kap.) angenommen, daß die Kernfäden, mit einer semipermeabeln Membran ausgestattet, vermöge eines Membranpotentials eine elektrische Ladung gegen die Kern- und Zellflüssigkeit annehmen und $\mathrm{da} B$ sie in einem durch Osmose entstandenen Potentialgefälle von ihrem ursprünglichen Orte im alten Eikern ( ̈̈quatorialplatte) nach den Orten der Tochterkerne, wo sich die Centrosomen befindeu, hingetrieben werden. Der Vorgang ist nach dieser Auffassung eine Elektrokinese. 
Erst nachdem wir dargelegt haben, welche Bedeutung das Nembranpotential der Zellen und ihrer Kerngebilde im Leben der Organismen gewounen hat, werden wir verstehen können, in welcher Weise sich diese früh erworbene und weiter vererbte Eigenschaft der Zellen bei der Differenzierung des M uskel-und Nervengewebes mit den Funktionen derselben verknüpft hat. An diesen Geweben sind die elektrischen Eigenschaften der tierischen Organe zuerst entdeckt worden, und in experimenteller Hinsicht mußten daher die an ihnen angestellten Untersuchungen auch in diesem Buche als Ausgangspunkt der Darstellung gewählt werdeu. An den sehr langgestreckten Muskelzellen, den Muskelfasern, und den langen Fortsätzen der Nervenzellen, den Nervenfasern, konnte vermittelst der oben behandelten Forschungen (1. bis 5. Kap.) die Grundlage der Membrantheorie gewonnen und das Vorhandensein eines elektrischen Membranpotentials abgeleitet werden.

Es ist in dell oben beschriebenen Untersuchungen (s. 9. Kap.) gezeigt worden, daß die ursprüngliche Bedeutung des elektroosmotischen Membranpotentials, welche in der Regulierung des Wassergehaltes der Zelle gefunden wurde, auch für die Muskelzelle gilt. Wir werden annehmen dürfen, daß diese Bedeutung auch dem Membranpotential der Nervenzelle und ibren Fasern zukommt. Indessen die Beziehungen dieser Membranpotentiale zu den Funktionen des Muskel- und Nervensystems sind engere und kompliziertere geworden. An den Nerren und Muskeln können wir durch die elektrische Untersuchung die Erregung und Reizleitung am genauesten beobachten. Die Erregung gibt sich an jeder Stelle derselben durch Abnahme des Membranpotentials zu erkennen, indem diese Stelle gegen eineruhende negativeSpannung annimmt, wodurch die "Aktionsströme" entstehen. Man ist daher imstande, die Reizleitung in diesen Organen mit Hilfe der angegebenen Instrumente zu verfolgen, ihre Geschwindigkeit und Dauer zu messen und festzustellen, daß der Reizzustand sich nach jedem Momentanreiz wellenförmig ausbreitet, ein Vorgang, den wir Reizwelle genannt hatten. Dieselbe kann langsamer oder schneller ablaufen, am langsamsten an den glatten Muskeln niederer wirbelloser Tiere und an ihren Nerven, am schnellaten an den quergestreiften Muskeln und Nerven der höheren Wirbeltiere. Die elektrische 
Zustandsänderung, die Reizwelle, geht in den Muskelfasern immer der Kontraktionswelle voraus; sie berleutet daher das erste Stadium der Gesamterregung des Muskels, welches das zweite, die Kontraktion, vorbereitet. Welche Beziehungen zwischen diesen beiden Vorgängen obwalten, haben wir oben (3. Kap.) ausführlich erörtert. In den Nervenfasern hat man neben der elektrischen Zustandsänderung bisher keinen anderen Prozeß bei der Tätigkeit nachweisen können, der sich als Folge hinzugesellte. Doch daß chemische Prozesse dabei auftreten, möchte ich als sicher ansehen.

Wir haben es oben (s. 7. Kap.) aus den Erscheinungen der inneren Polarisation und elektrischen Reizung wahrscheinlich zu machen gesucht, daß nicht nur die ganzen Muskelund Nervenfasern mit einer semipermeablen Plasmabülle begabt sind, sondern daß eine solche Membran auch den Fibrillen derselben zukommt. Die Fibrillen der Muskelfasern sind nun, wie wir wissen, diejenigen Gebilde, an denen sich der Kontraktionsprozeß vollzieht. Daher möchte ich an dieser Stelle noch kurz auf eine neuere Theorie hinweisen, welche man zur Erklärung dieses Prozesses aufgestellt hat. Es ist sehr wahrscheinlich geworden, daß die Protoplasmabewegungen, wie man siez. B. an Amöben beobachtet, durch Ïnderungen der Oberflächenspannung hervorgebracht werden (G. Quincke). Es ist daher auch versucht worden, die Muskelkontraktion durch dieselbe Energieform zu erklären, unter der Annahme, daß bei der Kontraktion eine Zunahme der Oberflächenspannung an der Oberfläche der Fibrillen stattfindet ${ }^{1}$ ). Auf diesen Gegenstand kann an dieser Stelle nicht weiter eingegangen werden und ich verweise auf die unten angegebenen Schriften. Aber es sei doch noch hervorgehoben, daß nach den gemachten Annahmen die elektrischen uñd mechanischen Prozesse sich beide an der gedachten semipermeablen Membran der Fibrillen abspielen würden.

Schließlich ist es in der lebenden Natur zur Entstehung eines Organes gekommen, das elektrische Ströme von erheblicber Stärke zu produzieren imstande ist. Die Natur hat, sozusagen, die Gelegenheit benutzt, um mit Hilfe des schon seit Urzeiten vor-

1) Bernstein, Die Energie des Muskels als Oberflächenenergie. Pflügers Arch. 85, 271-312. - Die Kräfte der Bewegung in der lebenden Substanz. Friedr. Vieweg \& Sohn 1902. 28 S. 
handenen Membranpotentials der embryonalen Zellen ein elektrisches Organ als Schutz- und Angriffswaffe zu konstruieren. Jede elektrische Zelle desselben besitzt keine größere Kraft als die quergestreifte Muskelzelle, aber vermöge der Entwickelung beim Wachstum haben sich die plattenförmigen elektrischen Zellen in großer Zahl zu Säulen aneinandergereilıt und geben daher, je nach ihrer Zahl, bei der Reizung durch Abnahme des Membranpotentials auf der Seite des Nervenfasereintrittes (Nervenendplatte) einen mehr oder weniger kräftigen Schlag, wie eine vielgliedrige galvanische Batterie (s. 6. Kap. S. 188).

Muskeln, Nerven und elektrische Organe sind bisher hauptsächlich diejenigen Objekte gewesen, an denen die elektrophysiologische Untersuchung die wichtigsten Resultate der Lehre von der Bioelektrizität gewonnen hat. Es wird weiterer Forschungen in diesem Gebiete noch an anderen und namentlich auch an pflanzlichen Organen bedürfen, um die Elektrobiologie im allgemeinen Sinne weiter auszubauen, deren Grundlagen zu geben in diesem Buche angestrebt worden ist.

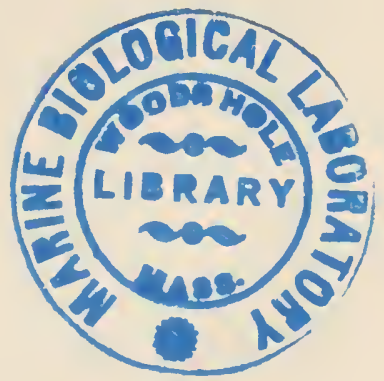




\section{Anhang.}

Die zur Untersuchung der bioelektrischen Ströme in neuerer Zeit hauptsächlich benutzten Instrumente sind erstens das K a pilla re le k trometer und zweitens das Saitengalvanometer. Sie sind deshalb hierzu besonders geeignet, weil sie die schnellen Schwankungen solcher Ströme bis zu einer gewissen Grenze gut anzugeben vermögen. Sie haben daher das Rheotom (s. S. 41) in diesen Untersuchungen zum großen Teil verdrängt; doch muß man bei vielen Aufgaben im Auge behalten, daß sie nicht wie das Rheotom den zeitlichen Ablauf der Ströme unmittelbar geben, sonder'u daß die erhaltene Stromeskurve erst einer Analyse durch Rechnung bedarf. Für die Untersuchung aller spontan ablaufenden bioelektrischen Ströme, wie z. B. derjenigen des Herzens usw., auf welche das Rheotom nicht eingestellt werden kann, sind sie aber unentbehrlich geworden.

Das Kapillarelektrometer, von dem Physiker G. Lippmann erfunden, ist ein in der Physik und physikalischen Chemie schon lange zur Messung schwacher elektromotorischer Kräfte benutztes Instrument. Es besteht im wesentlichen aus einem mit Hg gefüllten Glasrohre, welches unten in eine offene Kapillarspitze ausläuft, so daß bei etwa $70 \mathrm{~cm} \mathrm{Hg-Druck,} \mathrm{das} \mathrm{Hg}$ in der Kapillare einen Meniskus bildet. Das senkrecht stehende Rohr wird in ein mit verdünnter Schwefelsäure gefülltes Gefäß gesetzt, an dessen Boden sich eine Quecksilberschicht befindet. Das Glasrohr wird mit einem Druckapparat und einem Manometer verbunden. Durch Erhöhung des Druckes treibt man aus der Kapillarspitze die Luft aus und füllt den Raum derselben ebenfalls mit der Flüssigkeit an. Das Quecksilber der Röhre und das des Gefäßes wird mit deu Enden einer Kette durch einen Platindraht verbunden. Geht ein Strom durch das Elektrometer hindurch, so bewegt sich der Meniskus in der Kapillare nach oben, wenn er die Kathode, und nach unten, wenn er die Anode des Stromes bildet.

In der Fig. 60 sind die wesentlichen Teile des Kapillarelektrometers in der von Lippmann beschriebenen Form abgebildet. $B$ ist das mit der verdünnten Schwefelsäure $\left(1 / 3 \mathrm{H}_{2} \mathrm{SO}_{4}\right.$ und $\left.2 / 3 \mathrm{H}_{2} \mathrm{O}\right)$ gefüllte Gefäß, in welches die Röhre $A$ eintaucht. Der Schlauch, welcher diese Röhre mit dem Manometer verbindet, besitzt ein erweitertes Stück, das durch die Schranbe $V$ komprimiert werden kann, um den Druck zu erhöhen. Statt dessen kann man auch ein $\mathrm{Hg}$-Druckgefäß mit dem Schlauch 
durch ein T-Rohr verbinden, um durch Heben und Senken des ersteren den Druck zu variieren. Der Apparat ist zu physiologischen Zwecken mannigfach abgeändert worden.

Da es sich nur um geringe Verschiebungen des Meniskus handelt, so beobachtet man diese mit Hilfe des Mikroskops $M$. Zur Aufzeichnung von Kurven werden diese Verschiebungen projiziert und photographiert.

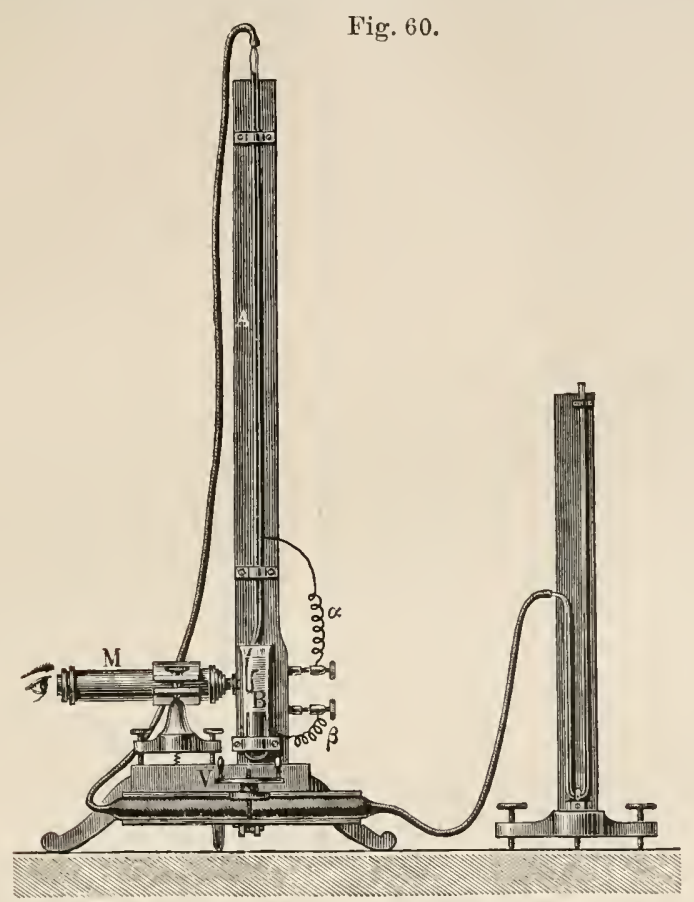

Kapillarelektrometer von Lip pmann.

Die Verschiebung des Meniskus beruht bekanntlich darauf, daß eine Polarisation zwischen $\mathrm{Hg}$ und der Flüssigkeit stattfindet. Es besteht nach der Erklälung vou v. Helmholtz ein Kontaktpotential zwischen Quecksilber und verdïnnter Säure, wodurch sich an der Grenze eine elektrische Doppelschicht bildet. Das Quecksilber ladet sich positiv und die Säure negativ. Die Oberflächenspannung des Quecksilbermeniskus, deren Kraft dem Druck entgegen nach oben gerichtet ist, wird durch diese elektrische Doppelschicht vermindert, weil in jeder Schicht die Abstoßung der positiven wie auch der negativen Teilchen gegeneinander die Oberfläche zu vergrößern strebt. Geht nun ein Strom in der Richtung hindurch, daß der Meniskus Kathode wird, so verringern die positiven Ionen des Elektrolyten, die sich an der Quecksilberoberfläche 
abscheiden, die Ladungen der Doppelschicht, die Oberflächenspannung des Meniskus nimmt zu, und er bewegt sich infolgedessen um ein Stück nach oben. Das Umgekehrte muß der Fall sein, wenn der Meniskus zur Anode des Stromes wird.

Der durch die Oberflächenspannung « zwischen Quecksilber und Flïssigkeit erzeugte, nach oben gerichtete Kapillardruck ist nach den Gesetzen der Kapillarität gleich $2 \kappa / r$, wenn $r$ der Radius der Röhre ist. Dieser hält dem Druck $p$ der Quecksilbersäule, der durch das Manometer angegeben wird, das Gleichgewicht. Wird " größer und hat sich der Meniskus nach oben bewegt, so kann man durch Steigerung des Druekes im Manometer denselben wieder auf den Nullpunkt zurïckbringen. Diese Drucksteigerung ist dann proportional der elektromotorischen Kraft des zugeleiteten Stromes. Hat die Kapillare eine konische Gestalt, so stellt sich das Gleichgewicht nach Zuleitung eines Stromes schnell dadurch ein, daß der Radius $r$ bei der Verschiebung sich vergrößert oder verkleinert. Wird « größer, so rückt der Meniskus nach oben, und da hierbei auch $r$ größ口r wird, so herrscht Gleichgewicht, sobald $\rho$ wieder gleich $2 \varepsilon / r$ geworden ist. Ungekehrt wird $r$ kleiner, weun " kleiner wird und der Meniskus sich nach unten bewegt. Innerhalb gewisser kleiner Potentialdifferenzen der zugeführten Ströme sind die Verschiebungen des Meniskus denselben nahezu proportional. Kurze konische Kapillaren reagieren daher auf Stromesschwankungen schnell und sind für physiologische Zwecke am besten geeignet. Bei einer Potentialdifferenz von 0,95 Volt erreicht der Meniskus das Maximum der Verschiebung nach oben und kehrt bei weiterer Erhöhung derselben wieder nach dem Nullpunkt zurïck. Daraus folgt, daß das Kontaktpotential $\mathrm{zwischen} \mathrm{Hg}$ und der verdünnten Schwefelsäure 0,95 Volt beträgt. Die zugeleiteten Ströme dürfen diese Kraft nicht viel überschreiten, weil dann elektrolytische Abscheidungen auftreten. Die Bewegung ist eine vollkommen aperiodische, d. h. es treten keine Eigenschwankungen um den Ruhepunkt ein.

Die Projektion und photographische Aufzeichnung geschieht nach bekannten optischen Methoden. Das Bild der Kapillare wird auf einen Schirm entworfen, welcher einen senkrechten feinen Spalt besitzt. Der Meniskus wird auf eine Stelle des Spaltes scharf eingestellt, so daß ein Teil des spaltes durch das $\mathrm{Hg}$ der Kapillare gedeckt ist, während durch den anderen Teil das Licht frei hindurchgeht. Bringt man dicht hinter dem Spalt einen um eine senkrechte Achse rotierenden Zylinder an, welcher mit photographischem Papier oder Film überzogen ist, so wird die Bewegung des Meniskus auf demselben als Kurve aufyezeichnet. Schirm und Zylinder müssen lichtdicht verbunden sein. Statt dessen hat man auch eine photographische Platte, mit einem Pendel (Burdon-Sanderson) oder einem Fallapparat (Garten) verbuuden, hinter dem Schirm vorbeibewegt. Mit Vorteil kanu man auch mit Hilfe eines rotierenden Spiegels das durch eine große Linse entworfene Bild des Spaltes auf eine photographische Platte einer Kamera werfen und das Bild des Meniskus über dieselbe fortbewegen (Bern- 
stein u. Tschermak). Nach letzterer Methode ist die Fig. 15 (S. 52) erhalten worden. Zugleich lassen sich Bewegungen der untersuchteu Organe (Muskeln usw.) durch vor den Spalt gestellte Hebel verzeichnen. Die Zeitschreibung geschieht durch Aufzeichnung von Federschwingungen; Reizmomente und andere Einwirkungen auf die Organe werden durch Signale vor dem Spalt angegeben.

Nach Berechnungen von Burch und yon Einthoven verfährt, man bei der Analyse der Kapillarelektrometerkurven folgendermaßen : Aus den Ordinaten der gezeichneten Kurve $y_{m}$ kann man die wirklichen Ordinaten der Stromeskurve $y_{b}$ durch Rechnung finden, wenn man eine Konstante $c$ bestimmt hat, welche die Schnelligkeit ausdrïckt, mit der der Meniskus den Stromesschwankungen folgt, und wenn man die Geschwindigkeit mißt, mit welcher die gezeichnete Kurve in dem Zeitmoment $t$ steigt oder sinkt, in welchem sie die Ordinate $y_{b}$ erreicht.

Um die Konstante $c$ zu finden, muß man eine Eichungskurve durch Schließen eines konstanten Stromes (unter 0,95 Volt) aufnehmen. Diese steigt in einer logarithmischen Form, erst schnell, dann langsamer, konvex nach oben gerichtet, zu einem Maximum an, das $y^{\prime}$ heißen möge. Dann hat man für $c$ die Formel:

$$
\frac{1}{c}=\frac{t \cdot \log e}{\log y^{\prime}-\log \left(y^{\prime}-y\right)}
$$

( $e$ ist die Basis der natürlichen Logarithmen, $\log e=0,4343$ ). Aus mehreren Werten von $y$ muß man einen Mittelwert von $c$ berechnen.

Die Geschwindigkeit, mit welcher die Elektrometerkurve steigt oder fällt, wird durch den Differentialquotienten derselben, $d y / d t$, ausgedrückt.

Für die Ordinaten der zu berechnenden Kurve hat man dann die Formel :

$$
y_{b}=y_{m}+\frac{1}{c} \cdot \frac{d y}{d t}
$$

Die für jeden Zeitpunkt $\mathrm{zu}$ berechnende Ordinate ist also gleich der beobachteten Ordinate für diesen Zeitpunkt plus oder minus einer Größe, welche gleich dem reziproken Werte der Eichungskonstante $c$ mal dem positiven oder negativen Werte von $d y / d t$ zur Zeit $t$ ist. In dem aufsteigenden Teil der Elektrometerkurve ist $d y / d t$ positiv, in dem absteigenden Teil derselben dagegen negativ. In dem ersteren Teil läuft die berechnete Kurve höher, in letzterem tiefer als die Elektrometerkurve. Im Maximum der letzteren, wo $d y / d t=0$ ist, schneidet sie dieselbe (s. Fig. 15).

Das Saitengalvanometer, von Einthoven ${ }^{1}$ ) konstruiert, beruht auf dem Prinzip des Deprez-d'Arsonvalschen Spulengalvano-

1) Ann. d. Phys. (4) 12, 1059 (1903). Onderzoekingen gedaan in het physiolog. Laborat., Leiden 1907. 155 S.

Bernstein, Elektrobiologie. 
meters. Statt der drehbaren Spule befindet sich im Magnetfelde eine feine leitende Saite, entweder ein versilberter Quarzfaden oder sehr feiner Platindraht, durch welche der zu messende Strom geleitet wird. Gehen die Magnetkraftlinien durch die an beiden Enden befestigte Saite senkrecht hindurch, so erleidet sie bei Durchleitung eines Stromes eine gegen erstere senkrecht gerichtete Ablenkung. Diese wird mit Hilfe eines Mikroskops beobachtet oder zur Aufzeichnung auf einen Spalt eines Schirmes, senkrecht gegen denselben gerichtet, projiziert,

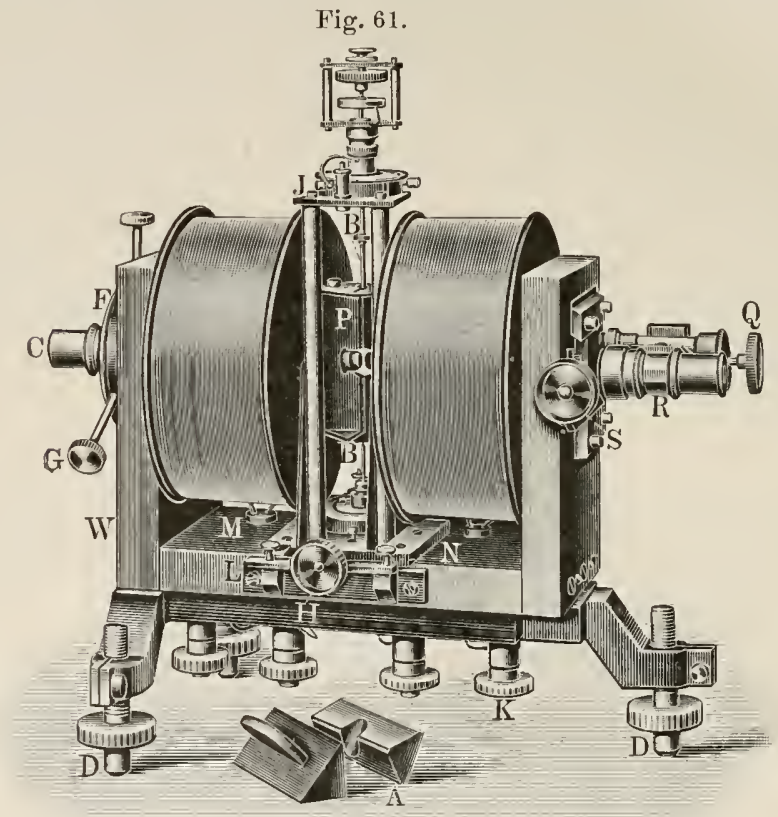

Saitengalvanometer von Einthoren (nach Konstruktion Edelmann).

so daß die Bewegungen der Saite in derselben Weise, wie oben beschrieben, photographisch registriert werden können.

In der Fig. 61 ist das von Edelmann hergestellte Saitengalvanometer abgebildet. Es besteht aus einem hufeisenförmigen Elektromagneten, dessen Pole mit den zwei schneidenförmigen Polschuhen $P$ belegt sind. Zwiscben diesen befindet sich der Fadenträger, der in Fig. 62 besonders abgebildet ist. Die Saite oder der Faden wird zwischen den Stiften $h h$ befestigt. Diese sind durch Mikrometerschrauben verschiebbar, um die Saite richtig einzustellen. Der Fadenträger wird mit seiner Grundplatte C (Fig. 62) in einen Schlitten des Gestelles eingeschoben. Die Saite wird in die Mitte des schmalen Spaltes zwischen 
den Polen eingestellt. Polschuhe und Elektromagnetkerne sind durchbohrt, um ein Beleuchtungsrohr $C$ (Fig. 61) und einen Mikroskoptubus $R$ einzuschieben. Dem Faden kann durch die Mikrometerschraube bei $k$ (Fig. 62) eine bestimmte Spanung gegeben werden. Die Länge der Fäden für schnell schwankende Ströme wechselt zwischen 32 und $87 \mathrm{~mm}$. Bei gewisser Länge, Dicke, Elastizität und Spannung der Saite kann die Bewegung derselben nahezu aperiodisch gemacht werden. Die Schlußplatten $A$ (Fig. 61) dienen dazu, um nach Einsetzung des Fadenträgers den Raum, in dem sich der Faden befindet, dicht abzuschließen.

Aus der gezeichneten Kurve die wirkliche zu berechnen, ist beim Saitengalvanometer viel schwieriger als beim Kapillarelektrometer, da die Bewegung der Saite eine elastische ist. Auch kann diese Bewegung nicht so aperiodisch gemacht werden wie die des $\mathrm{Hg}$-Meniskus, und daher treten oft Eigenschwingungen der Saite auf. Dagegen reagiert die Saite schneller als der $\mathrm{Hg}$-Meniskus und gibt daher Kurven, welche den wirklichen näher kommen, wie z. B. die doppelphasigen Aktionsströme. Für langsame elektrische Schwankungen muß die Saite schwächer, für schnelle stärker gespannt werden.

Einthoven hat eine Formel entwickelt, nach welcher die Analyse der gezeichneten Kurven zur Berechnung der wirklichen Kurven ausgeführt werden soll. In dieser Formel kommt nicht nur die Steilheit (erster Differentialquotient) der gezeichneten Kurve vor, sondern auch die Änderung dieser Steilheit (zweiter

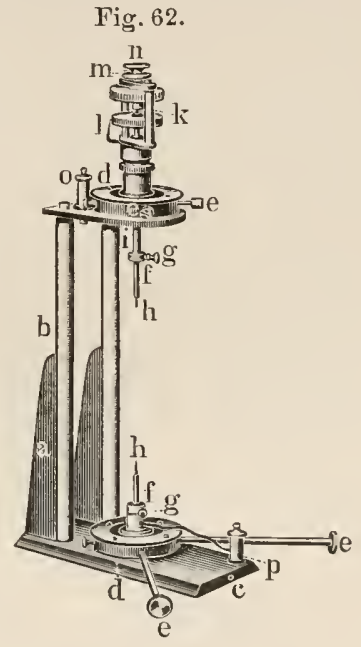

Fadenträger des Saitengalvanometers. Differentialquotient) in jedem Zeitmoment. Außerdem ist mit Bilfe einer Eichungskurve für jede dieser Größen eine besondere Konstante zu berechnen. Die Messungen, welche hierzu erforderlich sind, sind daher schwieriger und weniger zuverlässig als diejenigen, welche an einer Kapillarelektrometerkurve vorzunehmen sind. Nan wird demnach gut tun, bei Untersuchungen, die eine genauere Analyse erfordern, vorläufig das Kapillarelektrometer $\mathrm{zu}$ benutzen. Auch kann füir mancherlei Untersuchungen das Rheotom immer noch mit Vorteil gebraucht werden, da es ohne weitere Analyse den zeitlicheu Verlauf der Ströme unmittelbar ergibt.

Das Saitengalvanometer ist ein Amperemeter, während das Kapillarelektrometer ein Voltmeter ist. Es hat also letzterem gegenüber den Nachteil, daß man mit ihm nicht elektromotorische Kräfte direkt messen kann und daß Änderungen der Widerstände im Kreise, die bei vielen physiologischen Vorgängen eintreten können, einen EinfluB auf das 
Resultat haben. Von Cremer ${ }^{1}$ ) ist daher ein Saitenelektrometer konstruiert worden, in welchem die Saite zwischen zwei Polplatten ausgespannt ist. Man verbindet die Pole der Kette mit den beiden Polplatten und ladet die Saite mit einem hohen konstanten. Potential. Dieses Instrument hat freilich nicht die große Empfindlichkeit des Saitengalvanometers, doch ist es zur Untersuchung stärkerer Ströme wie der der elektrischen Organe gut geeignet (s. Fig. 39, S. 116) und bei sehr großen Widerständen dem Saitengalvanometer überlegen. Cremer ${ }^{2}$ ) hat ferner in den Kreis des Saitengalvanometers zur Aufnahme des Elektrokardiogramms einen Kondensator eingeschaltet, welcher den Vorteil gewährt, daß Ungleichartigkeiten der auf die Haut aufgesetzten Elektroden und der abgeleiteten Hautstellen keine Ablenkungen geben, während alle Potentialschwankungen, wie die des pulsierenden Herzens, schnell und infolge stärkerer Dämpfung aperiodisch verzeichnet werden.

In letzter Zeit hat man auch sogenannte Oszillographen nach dem Prinzip des Saitengalvanometers koustruiert. Statt der Saite befindet sich bei jenen eine feine gabelförmige Schlinge im Magnetfelde, welche sich wie eine Drehspule um ihre Längsachse dreht und mit einem Spiegelchen zur Reflexion eines photographisch zeichnenden Lichtstrahles versehen ist.

Von Bernstein ist der Vorschlag gemacht worden, den Kathodenstrahl als Indikator für die Oszillationen der bioelektrischen Ströme zu benutzen, da dieser mit absoluter Genauigkeit den Stromesschwankungeu folgen würde. Es ist aber bis jetzt nicht gelungen, Versuche in dieser Richtung mit Erfolg auszuführen.

1) Münch. med. Wochenschr. 1907, Nr. 11.

$\left.{ }^{2}\right)$ Zeitschr. f. Biol. 47, 137. 


\section{NAMEN- IND SACHREGISTER.}

A bierhalden 98. Aktionsströme 47.

Alterationstheorie 20 .

Anfangszuckung 149.

Ar'renius 21, 88.

Bernstein 41, 48, 51, 71, 85, 96, 118 , $131,134$.

Biedermann 100.

du Bois-Reymond 1 u. ff., 18, 45, 86, $110,140$.

Boruttau 77.

Braun 27, 119.

v. Brïicke 85,86 .

Buchanan 73 .

Burch 74, 83, 135.

Burden-Sanderson 70, 105, 177.

Clausius 31.

Cremer 95, 99, 113, 115, 146.

Demarkationsstrom 5, 17.

Differentialrheotom 41 .

Diffusionszylinder, -potential 33 .

I)ionaea muscipula 176; Ströme 178.

Ditler 73,81 .

Doppelschicht, elektrische 38 .

Drüsenstrüme 151.

Finthoven $49,79,81,83,86$.

E]ektrisches Organ 108; Bau desselben 110, 113; Schlag desselben 113 u. ff.; Theorie des Schiages 121 ; thermisches Verhalten desselben $116 \mathrm{u}$. ff.

Elektroden, unpolarisierbare 3, 4 .
Elektrokardiogramm 83.

Elektrokinese 158, 181.

E'lektromutorische Kraft des Muskelstromes 6 ; Messung 7 .

Elektruosinose 157.

Elektrotonische Ströme 131.

Elektrotonus 130; Kat-, An- 135.

Energie, freie 23; elektrische 26.

Engelmann 16, 60, 85, 107, 153.

Erregung, elektrische 135; Gesetzderselben 140, 148; Einfluß der Winkelrichtung des Stromes 150. Erregungswelle 40 .

Fick 65, 71, 141.

Freundlich 158.

Fuchs 77, 85 .

(íaleotti 95 .

Galvani 1; Zuckung ohne Metalle 10.

Garten 56, 57, 73, 86, 113.

Gelatinierung, Gerinnung 184 .

Gibbs 21, 88 .

Gotch 74, 81, 105, 135.

II aber 96.

Hautströme 151.

Heidenhain 14, 71, 121.

Helmholtz 3, 4, 19, 21, 29, 48, 15\%.

Hering 107, 138.

Hermann 8, 15, 20, 42, 56, 68, 97, $105,131,153,180$.

Herzströme s. Muskelstrom.

Hittort 31,32 .

Höber 96, 98, 100, 101.

van't Hoff 153 . 


\section{$-\quad 214$}

Holmgren 86 .

Hoorweg 143.

Hoppe-Sieyler 67.

Horsley 81.

llumboldt, Alex. v. 2, 11, 108.

Ionen, Wanderung der 31, 32.

Jahn 26, 119.

Jeusen 61,64 .

I apillarelektrometer 206.

Mc Kendrik 86.

Kerufäden (Chromatosomen) 188; Gesclıwindigkeit derselben 194.

Kernleitermodell 132.

Kiernteilung (Karyokinese) 187.

Ketten, elektrische 21; exotherms, enlotherme $\Sigma t ;$ Konzentrations24; galvanische 24; osmotische Flïssigkeits- 34.

Kolloide, Verhalten im Potentialgefälle 182.

Kontraktionswelle $48,52$.

Kïster 81.

Kühne 86.

Iamausky 64.

luesser 153.

Lewandowski 80 .

Lipoidtheorie 102.

Jippmanu 49.

Iuchsinger 154.

Iufthermometer, elektrisches 123.

Iarchand 82 .

Marey 83, 112, 149.

Matteucci 2, 7, 14.

Membranen, semipermeable 88 ; elektrische Eigenschaften ders. 89, 16:.

Membranpotential 95, 105, 178.

Membrantheorie 87; elektroosmotische 162 .

Mimosa pudica 175 .

Nolekularspannung, -hemmung (PHïger) 138.

Mïller-Hettlingen 180 .

Iuskel, quergestreifter Skelett- 5; Bau des M. gastrocn. des Frosches 10; chemische Prozesse im Muskel $65 \mathrm{u}$. ff.
Muskelfaser, quergestreifte 7 ; glatte ¿4.

Muskelstrom, des Herzens 8..

Muskelton 71 .

Verust 21, 28, 34, 35, 144.

Nerven, Reizwelle des 74; doppelsimniges feitungsvermögen 19.

Nervenstrom 15.

Netzhaut 85 ; -ströne 86.

Neuron 86.

Nienlai 77,84 .

(Dker-Blom 4, 89.

Orbelli 155.

Ostwald $3,4,21,88,144$.

Overtou 98, 101, 147.

Perxin 158, 159.

Pfeffer 88, 92.

Pflïger 135, 136.

Phasengreuzkräfte 38 .

Piper 57, 73.

Planck 96.

Polarisation, innere तer Muskeln und Nerven 131.

Potential, Adsorptions- 158; Diffusions- 33.

Präexistenztheorie 46.

Quincke. 157, 199.

Ranvier 16, 104.

Reizbarkeit, Veränderungen der - im Elektrotonus 136.

Reizbewegungen an Ptlanzen 174.

Reizung, latente 53.

Reizwelle des Muskels 40; der Nerven 52.

Resorption, Resorptionszellen 155.

Rheotom s. Differentialrheotom.

Riesenfeld $37,145$.

Röber 153 .

Rosenthal 151.

\$achs 116.

Saitengalvanometer 240.

Schenck 64. 


\section{- $215-$}

Schönlein 69, 112.

Schwankung, negative 11 ; d. Muskulstromes 12; des Nervenstromes 17, 104; der elektrotonischen Ströme 134.

Sekretion, Sekretiınszellen 152.

Steines. 59, 86, 130.

'Temperaturkoeffizient der freien Energie 23.

Thomson 25.

Torpedo 108.

Traube, MI. 88.

T'schermak 51, 64, 70, 81, 96, 118.

Jexküll, v. 20.

Verzár 103.

Waller 83.

Tetanus 11; sekundärer 13; Ritter- Wasserbindung in den Zellen 165. scher üffnungs- 138.

Weiss 144.

Thermostrom des Muskels 96; des: Weyl 129.

Nerven 103.

Wiedemann $157,160$. 



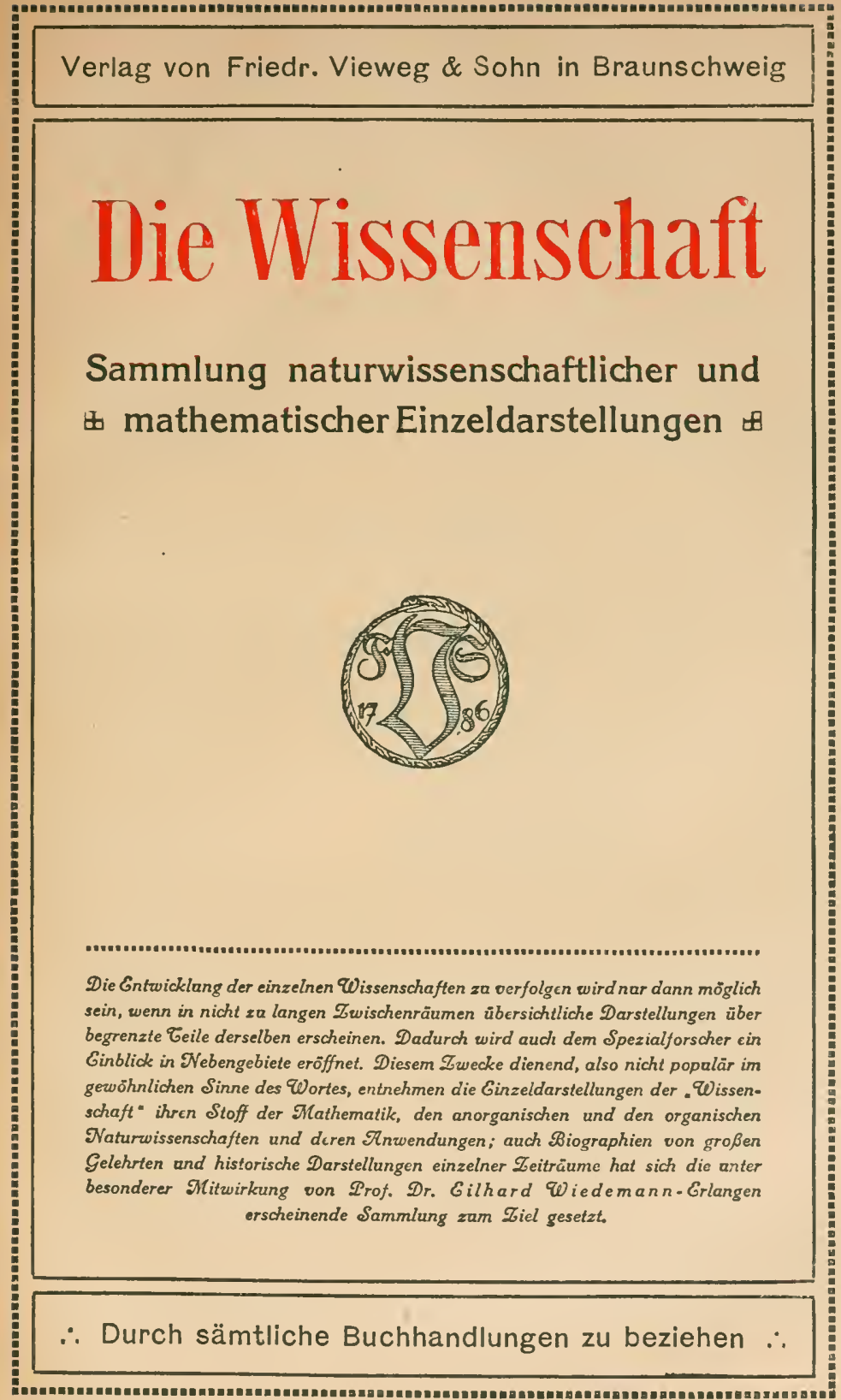

15000. 12. 11. 


\section{Verzeichnis der Mitarbeiter}

Dr. Otto Freiherr von und zu Auisess, München ..... Heft 4

Prof. Dr. K. Baedeker, Jena ........... „ 35

Prof. Dr. H. Baumhauer, Freiburg i. Schweiz . . . . . , 7

Prof. V. Bịerknes, Christiania ......... . ” 28

Mme. S. Curie, Paris . . . . . . . . . . . . ${ }^{\prime} 1$

Prof. Dr. B. Dessau, Perugia . . . . . . . . . 33

Prof. Dr. C. Doelter, Graz . . . . . . . . . . . . . ᄁ 13

Prof. Dr. B. Donath, Charlottenburg . . . . . . . , , 14

Dr. Wilh. R. Eckardt, Aachen . . . . . . . . . , 31

Privatdozenî Dr. Edwin S. Fausî, Straßburg. . . . . . " 9

Prof. Dr. Otto Fisclier, Leipzig . . . . . . . . . . . ” 18

Dr. 0tto Frölich, Berlin . . . . . . . . . ” 5

Privatdozent Dr. E. Gehrcke, Berlin . . . . . . . . " 17

Prof. Dr. F. E. Geinitz, Rostock . . . . . . . . . ." 16

Prof. Dr. Josef Ritter von Geitler, Czernowitz . . . . . . " 6

Prof. Dr. Sigm. Günther, München . . . . . . . . . . . . " 37

Prof. Dr. Hermann Gutzmann, Berlin . . . . . . . . . " 29

Prof. Dr. G. Jäger, Wien . . . . . . . . . " " 12

Prof. Dr. Albert Jesionek, Gießen ........... . " 32

Dr. phil. Walter von Knebel, Groß-Lichterfelde $(t) \ldots . . .715$

Prof. Dr. Hermann Kobold, Kiel . . . . . . . . . . " 11

Prof. Dr. Edm. König, Sondershausen . . . . . . . . " 22

Prof. Dr. J. P. Kuenen, Leiden . . . . . . . . . . . " 20

Prof. Dr. A. Lampa, Prag. . . . . . . . . . . . . . . . . " 42

Privatdozent Dr. M. Laue, München . . . . . . . . . , 38

Privatdozent Dr. G. F. Lipps, Leipzig . . . . . . . . , 10

Sir Oliver Lodge, Birmingham . . . . . . . . . . , 41

Prof. H. Mache, Wien . . . . . . . . . . . " 30

Prof. Dr. Joh. Bapt. Messerschmitt, München . . . . . . " 27

Aloys Müller, Bonn . . . . . . . . . . . . . . . . 39

Dr. Rob. Pohl, Berlin . . . . . . . . . . . , 34

Prof. E. Rutheriord, Montreal . . . . . . . . 21

Privatdozent Dr. 0tto Sackur, Breslau . . . . . . . . " " 24

Prof. Dr. K. Scheel, Berlin . . . . . . . . . . . . " 36

Ingenieur Fritz Schmidt, Berlin. . . . . . . . . . , 40

Prof. Dr. G. C. Schmidt, Königsberg . . . . . . . . . . . " 2

Prof. Dr. Julius Schmidt, Stuttgart . . . . . . . . . " 23

Prof. E. v. Schweidler, Wien . . . . . . . . . , 30

Prof. Dr. J. J. Thomson, Cambridge . . . . . . . $\left\{\begin{array}{lr}3 & 3 \\ 2 & 25\end{array}\right.$

Dr. P. Vageler, Königsberg i. Pr. . . . . . . .

Prof. Dr. A. Wangerin, Halle a. S. . . . . . . . . " 19

Prof. Dr. A. Werner, Zürich . . . . . . . . . ${ }_{n} 8$

(IVeitere Heite in ${ }^{\circ}$ Vorbereitung) 


\title{
Die W/issenschaft Sammmung naturuissenschalilu und mathematischer Ëinzeldarstellungen
}

\section{Untersuchungen über die radioaktiven Substanzen}

\author{
Von Mme. S. Curie. Übersetzt und mit Lite- \\ ratur-Ergänzungen versehen von W. Kaufmann. \\ Dritte Auflage. Mit 14 Abbildungen. VIII, 132 S. \\ 1904. Geh. M 3.-, geb. M 3.80.
}

Inlia itsverteichnis. Hinleitung. -1 . Kapitel. Radioaktivitat des Urans und Thors. Kadioaktive Mineralien. a) Becquerelatrahlen; b) Messung der. Strahlungaintensität; c) liadioaktivităt der Uran- und Thorverbiudungen; d) Ist die Radioaktivităt dor Atome ein allgemeines Phänomen? e) Ralioaktive Mineralien. - 2. KapiteL. Die neuen radioaktiven Substanzen. a) Untersuchungsmethoden; b) Polonium, Fadium, Aktinium; c) Spektrum des Radiums; d) A oscheiduug der neuen radioaktiven Substanzen; e) Polonium; f) Herstellung des reinen Rndiumchlorids; g) Bestimmung deo A tomEewichtes des Rałums; h) Eigenschaften der Radiumsalze; i) Fraktionierung gewöbnlichen Baryumchlorids. - 3. Kanitel. strahlungderneuen radioaktiven subatanzen. a) Methodeu zur Unterstchung der Strahlen; b) Energie der Strahlung; c) \%usammengesetzte Natur der Strahlung; d) Wirkuug des Msgnetfeldes; e) Ablenkbare p-Strahlen; f) Ladung der ablenkbareu strahlen; g) Wirkung des elektrischeu Feldes auf dio ablenkbaren B-Strahlen des Radiums;

h) Verhältnis ron Ladıung zur Nasse eines vom Radium emittinrteu neKativ geladenen Teilchens; i) Wirkung des Magnetfeldes aui die $\alpha$-strahlen; k) Wirkung des Magnetfeldes auf die Strahlen anderer radio-

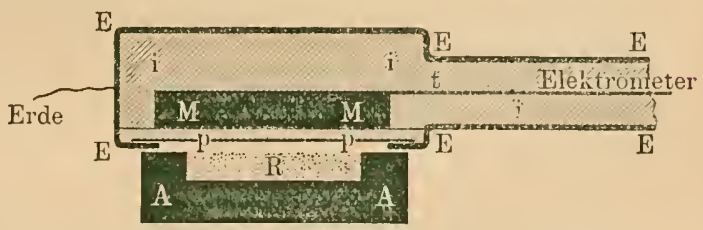
aktiver Substauzeu; 1) Verhältuio der ablenkbaren $\beta$-Strahlen in der Radiumstrahlung; m) Durchdringungsvermögen (er Strablung der radioaktiven Substanzen; n) Ionisierende Wirkung der Radiumstrahlen auf isolierende Flussigkeiten; 0) Versehiedene Wirkungen und Anwendungen der ionisierenden Wirkung der Strahlung radioaktiver Körper; p) Fluoreszenz- und Lichtwirkungen; q) Entwlckelung von Wärme durch Radiumsalzc; r) Chemische Wirkungen der neuen radioaktiven Substanzen. Fărbungen; s) Gaseutwickelung in Gegenwart von Radiumsalzen; t) Entstehuug ron Thermolumineszenz; u) Radiographien; v) Physiologische Wirkungen; w) Wirkung der T'emperatur auf die Strahlung. - 4. Kapitel. Induzierte Radioaktivltat. a) Mlitteilung der Radioaktivität an urspranglich inaktive Substanzen; b) Aktivierung in gexchlossenem Gefaß; c) Rolle der Gase bei den kirscheinuugen der induzierten Radioaktivitut. Emanation; d) Entaktivierung fester aktivirter Korper in freier Luft; $\theta$ ) Entaktivierung in geschlossenem Gefäß; Zerstörungsgeschwindigkclt der Emanation: f) Natur der Emanation; g) Anderung der Aktivität aktivierter Flìssigkeiten und radiumhaliger Lösungen; b) Theorie der Radioaktıvitä; i) Andere Form induzierter R ‘dioaktivität; k) Langeam entstehendo induzierte Radioalitivltat; 1) Induzierte Radioaktivitat auf mit Radium zusammen gelösten Substanzen; m) Zerstreuung radioaktiven Staubes und induzierte Aktivitä dea Laboratoriums; n) Aktivierung olıne Mitwirkung radioaktiver Substanzen; o) Änlerung der Aktivităt radioaktiver hörper; Wirknng der Auflosung; p) Änderung der Aktivität radioaktiver Körper; Wirkung der Erhitzung; q) Theoretisclio Deutung der Aktivität8anderungen der Kadiumsalze durel Auflösung oder Erhitzung. - 5. Kapltel. Natur uud Ursache dor Erscbeinungen der Radioaktiviıăt. 


\title{
Die Wissenschafit
}

\section{Die Kathodenstrahlen}

\author{
Von Dr. G. C. Schmidt, a. o. Prof. der Physik an der
} Unversität königsberg. Z we it e verbesserte und vermehrte Auflage. Mit 50 Abbildungen. VII, $127 \mathrm{~S}$. 1907. Geh. M 3.-, geb. M 3.60.

Inhaltsverzeichnis. Kinleitung. 1. Ka pitel. Das Wesen des Lichtes. Deräther. - 2. Kapitel. Neuere Ansichten uber die Leitung der Elektrizität durch Elektrolyte. 3. Kapitel. Apparate zur Erzeugung vou Kathodenstrahlen. - 4. Kapitel. Die Entladung in verdünnten Gasen. Die Kathodenstrahlen. - 5. Kapitel. Ältere Theorien über den kntladungsvorgang. - 6. Ka pitel. Ladung der Kathodenstrahlen. 7. Kapitel. Potentialgradienten und Kathodenfall in Entladungsröhren. - 8. Kapitel. Kathodenstrahlen im elektrostatischen Felde. - 9. Kapitel. Kathodenstrahlen im maguetischen F'elde. - 10. Kapitel. Lnergie und Geschwindigkeit der Kathodeustrahlen. - 11. Kapitel. Zeeman- Effekt. - 12. Kapitel. Liathodenstrahleu verechiedenen Ursprungs, - 13. Kapitel. Bestimmung von e und m. - 14. Kapitel. Scheinbare Masse. - 15. Kapitel. F'luoreszenzerregung und chemische Wirkung der Kathodeustrahlen. - 16. Kapitel. Reflexion, Absorption, Spektrum und Bahn der Kathodenstrahlen in einer Eutiadungsrohre. - 17. Kapitel. Kanalstrahlen. 18. Kapite L. SchluB. - Literaturabersicht.

\section{Aus den Besprechungen.}

Algemeines Literaturblatl.. „Die Aufklärungen über das scheinbar so rätselharte Verhalten der radioaktivcn Substanzen sinu rom Verfasser in ausnehıend interessanter und instruktiver Weise dargelegt und dürten wohl das weiteste Interesse tür sich in Anspruch nehmen. Die atomistische Theorie der Elektrizität, welche endlich verspricht, eiuen Einblick in das Wesen der elektrischen Erscheinungen zu geben und die Frage zu be-

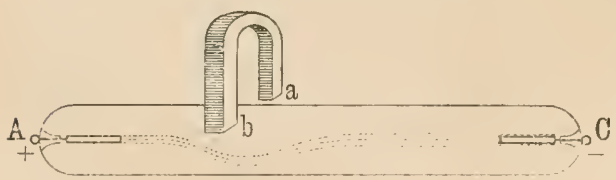
antworten, deren Lösung jabrhundertelang unmöglich schien: Was ist Elektrizität? basiert auf der Untersuchung der $\mathrm{Ka}$ tbodenstrahlen. Das für weitere Kreise rerständlich geschriebene Buch kann wärmstens emplohlen werden. Die Bebandlung des Themas ist einfach und gründlich; besonders ist auch die Beigabe einer großen Anzahl höchst klarer, schematischer Zeichnungen zu loben, welche die textliche Klarheit des Buches noch bedeutend erhöhen." 


\title{
Elektrizität und Materie
}

\author{
Von Dr. J. J. Thomson, Mitglied der Royal Society, \\ Professor der Experimentalphysik an del Universität in Cam- \\ bridge. Autorisierte Übersetzung von G. Siebert. \\ Zwe ite verbesserte Auflage. Mit 21 Abbildungen. \\ VIII, 116 S. 1909. Geh. M 3.-, geb. M 3.60.
}

Inhalt sverzeichnis. 1. Kapitel. Darstellung des elektrischen Feldes durch Kraftlinien, - 2. Kapitel. Elektrische und gebunuene Masse. - 3. Kapitel. Wirkungen der Beschleunigung der Faradayscheu Röhren. - 4. Ka pitel. Jie a tomistische Struktur der Elektrizităt. - 5. Ka pitel. Konstitution des Atoms. - 6. K a pitel. Radioaktivităt und radioaktive Substanzen. 7. Ka a itel. Materie und Äther.

\section{Aus den Besprechungen.}

Literarisches Zentralblatt. ${ }_{n}$ Eine Reihe geistvoller Vorträge, in welchen die Bedeutung der neuen Fortschritte in der Elektrizitätslehre für unsere Ansichten über die Konstitution der Naterie und die Natur der Elektrizität erörtert wird. Ihre Bedeutung liegt vor allem darin, daß sie eine auch weiteren Kreisen verständliche Verbindung zwischen den Maxwell-Faradayschen Vorstellungen und der modernen Elektronentheorie darstellen und dabei gleichzeitig des berühnten Verfassers eigene Anschaungen über den Aufbau der Atome entwickeln, wobei die radioaktiven Elemente eine besonders eingehende Besprechung erfahren. Die Ausführungen enthalten nur rereinzelte mathematische Ableitungen und könuen jedem Studierena'en emp̧ohlen werden."

Chemiker-Zeitung. ... ${ }_{n}$ Zu der Entwickelung der Elektronik, dieser neuen Disziplin der Pliysik, hat kaum jenıand mehr beigetıagen als J. J. Thomson... Es ist deshalb mit besonderer Freude $z u$ begrüßen, daß dieser bahnbrechende Forscher es unternommen hat, seine Ansiciten über die Natur der Elektrizität, über die Vorgänge, welche im elektrischen Felde stattfinclen, und iber den Zusammenhang zwischen elektrischer und gewöhnlicher Materie ${ }^{\text {in }}$ einer so anschrulichen und anregenden Weise darzulegen, da $B$ jeder Naturwissenschaftler, nicht nur der Physiker, das Buch verstehen kann und durch die Lektüre reichen Genuß und Gewinn haben wird. ... Für den Physiker, speziell für den Lehrer der Physik, eine Fundgrube anschaulicher Darstellungen und Gedankengänge. Für den Nichtphysiker eine Anleitung, nicht müheios, aber doch ohne das schwere Rüstzeug der höheren Mathematik, sich einen Einblick zu ver${ }_{8}$ chaffen in die Überlegungen, welche aus den Untersuchungen über Kathodenstrahlen, Röntgenstrahlen und Radioaktivität zu dem Begriffe des Elektrons, des Atoms der Elektrizität, getührt haben."

Verlag von Friedr. Vieweg \& Sohn in Branuschwelg 


\title{
Die physikalischen Eigenschaften der Seen
}

\author{
Von Dr. Otto Freiherr von und zu Aufsess, \\ Assistent für Physik a. d. Kgl. techn. Hochschule in München. \\ Mit 36 Abbildungen. X,120 S. 1905. Geh. M 3.-, \\ geb. M 3.60.
}

Inla ltsverzeichnis. Vorbemerkungen: Die Oberfäche eines Sees als Teil der Erdobertläche. Dichte des Wassers. Hydrostatigcher Druck. Kompressibilität des Wassers. - Erster 'I'eil. Mechauik. 1. Fortschreitende Wellen. 2. Stehende Wellen (Seiches). 3. Strömungen. - Zwoiter ' $\mathrm{I}$ 'eil: A k ustik. 1. Fortpflauzung des śchalles im Wasser. 2. Fortpflauzung des Schalles an der Oberflüche eines Sees. - Uritter 'I'eil: Optik. 1. Durchsiclutigkeit des Seewassers: A. Bestimmung der Durchsichtigkeit durch Versenken eines Gegenstandes; B. Bestimmung der Durchsichtigkeit durch Aufsuchen der Lichtgrenze im See. 2. Eracheinungen der Reflexiou, Brechung und Farbenzerstreung: A. Allgemeine Gesetze; B. Reflexionserscheinungen; C. Brechungserscheinungen; D. Dispersiouserscheinungen. 3. Selektıve Absorption des Lichter im Seewasser: A. Allgemeine 'l'heorie; B. Lichtabsorption in Wasser. 4. Polarisation des Lichtes im Wasser. 5. Lie Farbe der Seen. - Vierter Teil: 'T'hermik, 1. Thermometrie: A. Allgemeine Thermometrie; B. Spezielle Thermometrie: OberHächentemperaturen; Tiefentemperaturen; Fisverhältuisso. 2. Ka!orimetrie. Sohlukbemerkung. Literaturverzeichnis.

\section{Aus den Besprechungen.}

Blätter für höheres Schulwesen. „Die Darstellung ist ganz elementar und sehrklar gehalten. Der luhalt gliedert sich naturgemäß in die Mlechanik, Akustik, Optik und Thermik der physikalischen See-Erscheinungen. Besonulers interessant sind die Untersuchungen über den so viel diskutierten Grund der Verschiedenfarbigkeit der Seen. Die Erscheinungen des Wasserschattens werden mit dem Brockengespenst in zutreffende Parallele gestellt. Aber von dem allergrößten Interesse sind S. $63 \mathrm{fl}$. die Ausführungen über die Brechungsprscheinungen beim Übergange des Lichtes von Wasser in Luft. Es wird hier ganz elementar nachgewiesen, wie relativ und einseitig unsere Erkenntnis der Dinge ist. Wir sehen alle Gegenstände nur durch das Medium Luft, ein Wasserbewohner sieht dieselben Gegenstände durch das Medium Wasser ganz anders als wir, ja er sieht sogar Sachen, die wir als aus einem Stücke bestehend, als koutinujerliche Massen bezeichnen, in Stücke zerteilt!! Das Buch sei auch für die Schüler der obersten Klasse empfohlen."

Himmel und Erde. ${ }_{n}$ Was der Physiker vom weitverbreitetsten Stoffe auf unserem Erdball, dem Wasser, zu sagen weiß, ist fast lückenlos in dem A ufsessschen Buche zusammengefaßt worden. Wir erfaliren etwas über die Wellenbewegung an der Oberfläche, die Strömungen, Fortpflanzung des Schalles im Wasser, über die Durchsichtigkeit und die thermischen Verhältnisse. Besonders eingehend behandelt der Verfasser auf Grund eigener Versuche die Durchsichtigkeit und Farbe der Gebirgsseen, wobei er die Frage entscheidet, ob letztere ehemischer oder physikalischer Art ist. Wir empfehlen das Buch besonders allen denen, die es lieben, ihre Erholung in einer liebevollen Betrachtung der Natur zu suchen." 


\title{
Die Entwickelung der elektrischen Messungen
}

\author{
Von Dr. O. Frölich. Mit 124 Abbildungen. \\ XII, 192 S. 1905. Geh. M G.-, geb. M 6.80.
}

\begin{abstract}
Inhaltsverzeichnis. Die Mefinstrumente. Die Strommesser. a) Dio ersten Galvanometer. Oersted, schweigger. Uhm, Ampère, Biot-savart. Nobili, H. Davy, Faraday. Kalibrierung. Methode vou Petrina. Formel von Yoggendorff. b) Die Spiegelgalvanometer. Gauss. spiegelablesung. Gauss und W. Weber. Coulomb. Methodeu von W. Wober. Telo raphie und spiegelgalvanometer. Dămpfung. S'pätere Spiegelgalvanometer. spiegelgalvanometer mit Lrehspule. c) Galvanonoter mit direkter Ablesung und absoluteu Angaben. Hlelitromagnetische Wage. Sinusbussole. 'Taugenteniussolo. Pouillet. Helmboltz. Gaugain. Riecke. Absolutes Maßsystom. Torsions calvanometer von Siemens und Halske. Instrumente von Weston. Hlektrolytische Strommenser. d) Schalttafelinstrumente, Lirforclernisse von Schalttafelinstrumenten. ¿chalttafel-
\end{abstract} instrumente mit permanonten Magneten und beweglicher stromspule. Carpentier. Schaltafelinstrumente mit Eisenkeruen. Hitzdrahtiustrumente. e) (ialvanoskop. Anwondung für Telegraphio und Hessungen. f) Elektrodynamoneter und Wechselstrom. messer. Wlektrodynamometer von W. Weber. Spätere Llektrodyuamometer. Andere Instrumente für W ochselstrom. Techuischo Elektrodynamometer. Linergiemessung mittels Eilektrodynamometer. Neuere technische Elektrodynamometer. Herrarisinstrumente. S panung guesser. Ubersicht. Filektrostatische Spanuungsmesser. Kapillarelektrometer. Widerstandsa p parat $\theta$. Maßeinheiten. Widerstandsskalen. Kondengatoren. Selbstiud uktionsskalen. ApparatezurMessung maguetischer Eigenschaften. Elektrische Wärme. mos ser. Elektrizitätszäller. Uhrzähler. Motorzähler. Induktionszähler. Elektrische Registriorapparate. Hioktrische Regıstrierumen. Registricrung ejektri,cherVorgänge. Uszillographen. W'ideratandsmesser. Elektrische lies chwindig ke it $8 \mathrm{~m} \theta$ s $\mathrm{ser}$. Allgemeine Elektrizitatsg6sell schaft. - Die Meamethoden. Lie I ethoden der sirumuessung. Die Methoden der spanuung*messung. Spannungsmessung durch Strommessung.

Spannungsmessung mittels Normalelemente. Transformation der Spannung. Dio Mothouen der Widerstandsmessung. Methoden von W. Weber. Wheatstonesche Brücke. Formen der Meßbrücko. 'Temperaturmessung mittols Mebluricke. Sehr kleino Widerständo; Thomsonsche Meßbrucke. Sehr hohe IViderstände; Kabelme:sungen. Einfluß von Ladung und selbstinduktion. Einfluß von elektromotorischen Kraften; Widerstand von Zersetzungszelion, Batterien usw. Noumann. Kolnlrauech. Mance. Jutteroth. Frölich. Wehlerbestimmnngen. Messung des Widerstandes aus Strom und Spannung. Isolationswiderstand von Anlagen im Betriebe. Methoden zur Be. stimmung der Selbstinduktion. Methoden von Marwell. Neuere Methoden. Wien. Messungen für Ferusprechzwecke. Ule Methoden der Wechsolstrommessung. Zusammenstellung. R thckblick. 


\title{
Elektromagnetische Schwingungen und Wellen
}

\author{
Von Dr. Josef Ritter von Geitler, auBerordenth. \\ Professor der Physik an der k. K. Deutschen Universität Prag. \\ Mit 86 Abbildungen. VIII, 154 S. 1905. Geh. M4.50, \\ geb. M 5.20 .
}

Inhaltsverzeichnis. Einleitung. I. Kapitel. Theorieder Fernwirkungen. Isaac Newton. - II. Kapitel. Theorie der vermittelten Fernwirkung. I. Abschnitt: Michael Faraday. Das magnetische Feld, Das elektrische Feld. Der elektrische Strom. Das elcktromagnetische Feld. Die Induktionserscheinungen. Die Erscheiuungen der Selbstinduktion. Elektrische Schwingungen. Finige Grundbegriffe aus der Lehre von den Schwingungen. II, $\Lambda$ bschnitt: James Clerk Maxwell. Die kritische Geschwindigkeit. Elektromaguetische Lichttheorie. III. Abschnitt: Heinrich Radolf Hortz. IV. Absclunitt: Die weitere Entwickelung. Methoden zur Beobachtnng Hertzocher Wellen. Die elektromagnetischen Wellon und die Optik. a) Langste Wärmeund kurzeste elektrische Wellen. b) Nachahmung optischer Verouche mit Hertzechen Wellen. c) Optische Analogien von Versuchen mit Hertzacheu Wellen. d) Der Iuterferenzversuch von V. v. Lang. e) Spektralanalyse der elektromagnetischen Strahlung. f) Rolle des Leiters bei Drahtwellen. g) Die drahtlose Telegraphie. - Namenverzeichnis,

\section{Aus den Besprechungen.}

Annalen der Elektrotechnik. ...., Die rom Verfasser gewählte Art der Darstellung folgt der historischen Entrickelung des Gegenstandes bis in die

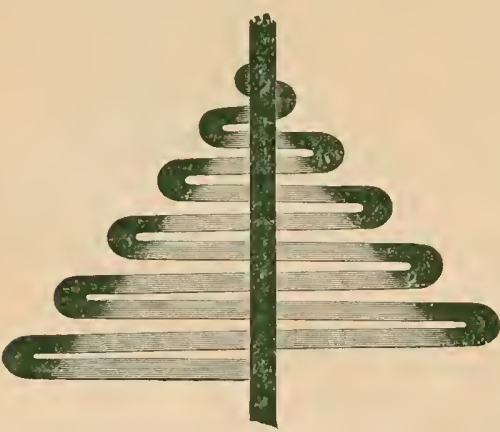
neveste Zeit und stellt an die mathematische Vorbildung seiner Leser nur die bescheidensten Ansprüche. Die Behandlung des Stoffes ist ausgezeichnet, die Gliederung klar und deutlich, die 86 gut ausgeführten Textfiguren unterstützen und erleichtern ganz wesentlich das Verständnis der für den Niclitphysiker immerhin schwierigen Materie. Da auch die Ausstattung und der Druck in gediegener Weise ausgeführt ist, so kann das Buch auf das wärmste empfoilen werden. Für deu Studenten der Physik und Elektrizitätslehre ist das Bändchen als erste Einführung in das genannte Gebiet rou großem Nutzen, es gibt aber auch dem gebildeten Nichtphysiker, besonders dem praktisclien Elektrotechnikir und Ingenjeur einen bequemen Überblick über die einschlägigen theoretischen Prubleme und deren experimentelle Lösung." 


\title{
Die neuere Entwickelung der Kristallographie
}

\author{
Von Dr. H. Baumhauer, Professor an der Universität \\ zu Freiburg 1. d. Schweiz. Mit 46 Abbildungen. VIII, \\ 184 S. 1905. Geh. M 4.-, geb. M 4.60.
}

Inhaltsverzeichnis. I. Abschnitt. Einleitung. Wesen und Definition eines Kristalls, Fliebende und flussige Kristalle. Zonengesetz und Gesetz der rationalen Achsenschnitte. Kristallographische Symbole. Linearprojektion, gnomonische und sphärische Projektion. Kohäsionsminima innerhalb der Kristalle. - II. A bschnitt. Kristallklassen und Pseudosymmetrie. Einteilung der Kristalle in 32 Klassen. Symmetrieelemente: Zentrum der Symmetrie, Symmetrieebenen, Deck- und Syiegelachsen. Kristallsysteme. Spezielle Ableituug und Besprechung der elnzelnen Kristallklassen. Ableitung derselben auf Grund der Deck- und Spiegelachsen. Übersicht tiber die 32 möglichen Kristallklassen. Pseudo sy mmetrische Kristalle. - III. A b s chnitt, Hirmittelung der Symmetrieverbältuisseder liristalle. Gonfometrische Untersuchung, $z$ weilereisiges Gowioneter. Pbysikalische Eigenschaften der Kristalle, insbesondere optisches Verhalten; Zirkularpolarisation optisch-einachsiger und $-\mathrm{zweiachsiger} \mathrm{Kristalle.} \mathrm{Yolare}$ Pyroelektrizität. Ätz-oder Lösuugsorscheinungen. Geometrische Auomalien (vizinale Flächen), Optische Anomalien. Anomale Ätzfiguren. Allgemeinere Bedeutung der Ätzerscheinungen. - IV. A bschnitt. Zwillingsbildung der Kris a 11 e. Zwillingsachsen $u . Z$ willingsebenen. Allgemeine Zwillingsgesetze. Ableitung der verschiedenen möglichen Fälle vou $Z$ willingsbildung. Deutung des Vorganges der Zwillingsbildung. Translationstlächen als Zwillingrebenen. Zwillinge von enantiomorplen Kiristallen. Begunstigung der Zwillingsbildung. Polysynthetische Verwachsung pseudosymmetrischer Kristalle, Mimesie. - V. Abschuitt. Flächenentwickeluug und Wachstum der Kristalle. Entwickelung der Kristallflächen innerhalb der Zonen. Gesetz der Komplikation. Beobachtungen an flächeureichen Zouen; primäre Reihen, sekundäre und tertiare Flächen. Raumgitter und regelmälige Punktsysteme. Klementarparallelogramm and Hăufigkeit einer Fläche. Eiuflul des Lősungsmittels auf die Form der sich ausscheidenden Kristalle. Untersuchungen uber das Wachstum der Kristalle. - VI. A bschnitt. Chemische Kristallographie. Isomorphie. Definition derselbeu. Morphotropie. Topische Achsen. P. $\nabla$. Groths neuere Auffassung der Kristallstruktur, Morphotropio und Isomorphie. Polymorphe (monotrope und enautiotrope) Modifiationen. Mischumgen isodimorpher Körper. Beziehuugen zwischen der chemischen Formel und dem Kristallsysteme einer Verbindung. - Anhaug. Kristallklassen, Namen und Symbole der Formen nach P. ז. Groths physilalischer Kristallographie. 


\title{
Meuere Anschauungen auf dem Gebiete der anorg. Chemie
}

\author{
Von Prof. Dr. A. Werner in Zürich. Zweite \\ Auflage. XV, 292 S. 1909. Geh. M9.-, geb. M10.-.
}

Inhaltsverzeichnis. I. Die Elemente. 1. Dcr Elementenbegriff. 2. Systematik. - II. Die chemischen Verbindungen. A. Allgemeiuer Toil. Jehro von der Valezz. I. Lintwickelungsgang der Wertigkeitslehre. IL Über die Valenzzahlen. 1. Die Hanptralenzzahl. 2. Die Nebenvaleuzzahl. 3. Die hoordinationszahl. 4. Die ionogene Nebenvalenzzabl. IIf. Úber die Valenzeinheiten. 1. Eiuleitung. 2. Definition von Haupt- und Neheuvalenzcn. 3. Die Valenzeinheit als gerichtete Kinzelkraft. 4. Über das Wesen der Hau t- und Nebenvaleuzen. Der ubereinstimmeude Charakter von Hauptund Nebeuvalen en. 5. Der elektrochemische Begriff der Hauptvalenz. 6. Der Affinjtätswert der Va.enzbindungen. IV. Schlubbetrachtungen über Affinität und Valenz. B. s ystem a ti s c h er 'T' il. I. Die Verbindungen crster Ordnung. 1. Vinleitung. 2. Nounenklatur. 3. Systematik. 11. Die Verbindungen höherer Ordnung. 1. Halogenosalze und analoge Verbinduugen. 2. Verbindungen höherer Ordnung mit Oxyden, Snlfiden usw.: Die Anlageruugsverbindungen; Jie kinlagerungsverbindungen. 3. Verbindungen höherer Ordnuug mit Nitriden, Phosphiden usw.: Anlagerungsverbindungen; Eiulagerungsverbindungen. 4, Verbindungen höherer Orduung mit Karbiden. 5. Verbindungen höherer Urdnung mit verschiedenen Mlolekulkomponenten: Anlagerungsterbindungen; Einlageruugsverbindungen. 6. Über mehrkeruige Mctallammoniake. 7. Über koordinativ ungesättigte Eiulagerungsverbindungen. $\%$. Die Koordiuationsverbindungen der Wasserstofiverbindungen. 9. Theorie der basen und Säuren, 10. Über die innercn Metallkomplexsalze. 11. Über Komplexveriıindungen nit negativen Zontralatomen. 12. Uber Nebenvalenzverbinduugen von Ëlementen. 13. Allgemeine Betrachtungen über die Bildung von Verbinduagen böherer Ordnung. III. Lehre vou der Isomerie bei anorgauischen Verbiudungen. 1. Polymerie. 2. Koordinationsisomerie. 3. Hydratisomerie. 4. Ionisationsmetamerie. 5. Salzisomerie. 6. Strukturisomerie. 7, Haumisomerie. 8. Valenzisomerie. 9. Unaufgeklärte Isomeriecrscheinungen.

\section{Aus den Besprechungen.}

Chemiker-Zeitung. ${ }$ Die zweite Auflage des eben genannten Buches, dessen erste Auflage den Lesern dieser Zeitung bestens empfohlen wurde, ist aus dieser durch eine eingehende Umarbeitung und Durcharbeitung unter Berücksichtigung des inzwischen neu gefundenen Tatsachenmaterials entstanden. Ein Hauptunterschied beider Auflagen besteht in der Anordnung. Während in dem früheren zweiten Hauptteile über Verbindungen erster Ordnung und in dem dritten Hauptteile über Verbindungen liöherer Orduung jedesmal die betreffenden Valenzfragen zunächst behandelt usd dann die Systematik der Stoffe gegeben wurde, sind jetzt beide Hauptteile vereinigt, wodurch es ermöglicht wurde, die Valenzfragen im Zusammenhange darzustellen. Meincs Erachtens hat das Werk dadurch an Klarheit und Übersichtlichkeit erheblich gewonnen. Und das ist gut. Werners gedankenreiche Darlegungen stürzen alte eingewurzelte Anschauungen und setzen Neves an ihre Stelle. Dem zu folgen, erfordert tüchtige Mitarbeit, und jede Erleichterung dabei wird vom Leser mit Dank entgegengenommen. Im speziellen Teile wird die Theorie der Hydrate, der Hydrolyse, der Ammoniumverbindungen besonderes Interesse erwecken."

Heinrich Biltz. 


\title{
Die tierischen Gifte
}

\author{
Von Edwin S. Faust, Dr. phil. et med., Privatdozent \\ der Pharmakologie an der Universität StraBburg. XIV,248S.
} 1906. Geh. M 6.-, geb. M 6.80.

Inhaltsverzeicanis. Vorwort. - Einleitung. Zweck und Nutzen einer zusammenfassenden Behandlung der tierischen Gifte. Begriffsbestimmung. Was gehort zu den tierischen Giften? "Aktiv" und "passiv" gifige l'iere. Eigentliche Gifte und gelegentlich die Gesundheit schädigende tierische Produkte. Zoonosen. Historisches über tierische Gifte. Aberglaubea. Entwickelung unserer Kenntuisse über dieselben. Praktische Bedeutung der tierischell Gifte für die sie produzierenden Tiere. Praktische Bedeutung der tierischen Gifte für den Heusclen: Giftmord, Selbstmord, Hinrichtung von Verbrechern; Verweudung zur Herstellung von Pfeilgiften; Medizinale Vergiftungen durch tierische Gifte; Verwundung durch Bisse oder Stiche giftiger Tiere; 'Therapeutische Verwendung tierischer Gifte. Systematik. - Wirboltiere, Vertebrata. Säugetiere, M a m malia. Uitnithorhynchus paradoxus, Platypus. Das Adrenalin, Jie Gallensäuren: Die pharmakologischen Wirkungen der Gallensäuren. Schlaugen, Ophidia. Giftschlaugen, Thanatophidia. Übersicht. Historisches. Begrifisbestimmung. "Giftige und $e^{\text {ungiftige }}$ "Schlangen. "Verdächtige Schlangen". Systematik und geographische Verbreitung der Gittschlangen. A. Colubridae venenosae, Giftnattern. B. Viperidae. Soleuoglypha, Köhrenzähner. Die Giftorgane der Schlangen. Dio physiologische Bedeutung des Schlangengiftes. Über die Natur des Schlangengiftes. Wirkungen der Schlangengifte. Natürliche Immunität gewisser Tiere gegen schlangengifte. Künstliche oder experimentelle Immuuisierung gegen Schlangengifte. Angebliche Immunität gewissex Kategorien von Menschen gegen Schlangengift. Therapie des schlangenbisses. Prophylaxe. Eidechsen, Sauria. Amphibien, Lurche; Amplibia. 1. Orduung: Aura, schwanzlose Amphibien. 2. Ordnung: Urodela, geschwänzte Amphibieu. Fis che, $\mathrm{Pi}$ «es. I. Giftfische. A. Fische, welche durch Bib vergitten. B. Fische, welche durch Stichwunuen vergiften. C. Fische, welche ein giftiges Hautsekret bereiten. II. Gift:ge Fische. III. Vergiftung infolge des Geuusses durch postmortale Verinderungeu gesundheitsschädlich oder gitig gewordener Fischo. - Wirbellose Tiere, Avertebrata, M uscheltiere, Lamellibrauchiata. Gliederfǘror, Arthropoda. 1. Klasse: Spinnentiere, Arachnoides. a) Ordnuug Scorpionina. Arthrogastra, Glioderspinuen. b) Uränung Araneina. c) Urdnung Solifugae, Walzenspiunen. d) Urdnung Acarina, Milben. 2. Klasse: Myriapoda, Tausendfüßler. a) Urdnung Chilopoda. b) Ordnung Chiloguatha s. Diplopoda. 3. Klasge: Hexapoda, Insekteu. a) Urdnuug Hymenoptera, Hauttlügler. Familie Apidae, Bienen. Familie Formicidae, Ameisen. b) Orduung Lepidoptera, Schmetterlinge. c) Ordnung Coleoptera, Käfex. d) Ordnung Orthoptera, Geradflügler, Schrecken. e) Ordnung Diptera, Zweiflügler, Fliegen. Crustacea, Masseuvergiftungen durch Crangon vulgaris. Würmer, Vermes. Plathelminthes, Plattwürmer. Nematlelminthes, Kundwürmer. Annelida, Ringelwürmer. Stachelhäuter, Echinodermata. Seesterne, Asteroidea. Seeigel, Echinoidea. Seowalzen, Seegurken, Holothurioidea, Coelenterata (Zouphyta), PfIanzentiere. - Namenverzeichnis. - Sachregister.

\section{Aus den Besprecliungen.}

Repertorium der Praktischen Medizin. ... ${ }_{n}$ Wir haben bis jetzt ein Buch, das in dieser austührlichen Weise vom Standpunkte des Zoologen, Pharmakologen, Physiologen und Pathologen die tierischen Gitte einer Betrachtung unterwirft, nicht gehaut. Ganz besonders wird uns das Kapitel über Schlangen und Schlangengifte, vor allem auch der physiologische und dann der therapeutische Teil interessieren, wobei der Autor alle Methoden eingehend beschreibt nnd auf ilıren Wert prüft. Einen wertvollen Beitrag bieten die Darlegungen über Immunität und Immunisierung. "... 


\title{
Die psychischen Maßmethoden
}

\author{
Von Dr. G. F. Lipps, Privatdozent der Philosophle \\ an der Unlversität Lelpzig. Mit 6 Abbildungen. X, 151 S. \\ 1906. Geh. M 3.50, geb. M 4.10.
}

Inhaltsverzeichnis. Erster Abschnitt. Psychologienad Naturwizaen$8 \mathrm{chaft}$. 1. Die empirische und die philosophi-che Weltbetrachtung. 2. Die Jiewußtseinsinhalte. - Zweiter Abschnitt. Jie Wahroheinliehleitslehre. 3. Gewibheit und Wahrseheinlichkeit. 4. Die Wahrseheinlichkeitsbestimmung. - Dritter Abschnitt. Die Mabbostimmungen bej der Bertieksichtigung subjektiver Faktoren in Bereiehe der naturwissenschaftlichen Forschug. 5. Die Beobachtungsfehter. 6. Die Uagenauigkeit der Sinneswahrnehmung und die sonstigen subjektiven Faktoren. - Vierter Abschnitt. I)ie psyehophysischeu Mabuethoden, 7. Der uaturphilosophische Standpunkt Fechners nad das psychophysische Grundgesetz. 8. Das Mals der kimpfindlichkeit. 9. Nie Methode der eben merkliehen Unterschiede. 10. Die Methode der mittleren Fehler. 11. Die Methode der richtigen und falschen Fiille. 12. Die Nothode der mittleren Alstufungen, 13. Die Booluachtungsreihen. 14. Das Fehlergesetz. 15. Die Mittelwerte der Beoba.intungsreihen. - Funfer Abschnitt. Nas psychisehe Ma \&. 16. Dio dureh techner begrtudete Auffassungsweise des psychischen Mlabes. 17. Ordnen und Messen. - Seehster Abachuit. Wie Metloclea der psychischou Abhäugigkeitsbestimung. 18. Lie Bestimmung des Graules der Abhängigkeit. 19. Der 'iypus der Beobaclituvg:reihe. 20. Wie Zeriegung der Beobachtongsieihe in Komponenten und die Bestimmung der Unterschiedaschwelle. - An ha $\mathbf{n g}$. 21. Die Berechuung der Mittelwerte. - Literaturverzeichnis. - Register.

\section{Aus den Besprechungen.}

Literarisches Zentralblatt. „In der Literatur begegnet man noch so oft unklareu und fehlerhatten Anschauungen über die psychischen Maßrnethoden, daB eine umfassende monographische Darstellung der letzteren sicher einem Bedürfnis entspricht. G. F. Lipps gibt nun in der Tat eine Monographie, welche auch zur ersten Einführung in das Gebiet sich recht gut eignet. Er hat sich dabej weiter die doppelte Aufgabe gestellt: einesteils zu zeigen, da B die von Fechner in Anlehung an das gewöbnliche Fehlergesetz begründeten Maßmethoden unzureichend sind, und anderenteils den Weg anzugeben, aut dem man ohne Voraussetzung eines bestimmten Fehlergesetzes zu einer allen Bedürfnissen der experimentellen Psychologie genügenden Methode der Maßund Ablängigkeitsbestimmung gelangt. An den Ausfall dieses letzteren Versuches knüplt sich in wissenschaftlicher Beziehung das Hauptinteresse an der Abhandlung des Verfassers."

Physikalische Zeitschrift. ${ }_{n}$ Wer den Wunsch hegt, einen Überblick über das Rüstzeug der messenden Psychologie $z u$ gewinnen, dem wird das vorliegende zehnte Heft der Viewegscben Sammlung "Die Wissenscbaft" sehr willkommen sein. Das Buch wird sich bald einen größeren Freundeskreis erwerben." 


\title{
Der Bau des Fixsternsystems
} mit besonderer Berücksichtigung der photometrischen Resultate

\author{
Von Dr. Hermann Kobold, außerordentl. Professor \\ an der Universität und Observator der Sternwarte in Klel. \\ Mit 19 Abbildungen und 3 Tafeln. XI, 256 S. 1906.
} Geh. M 6.50, geb. M 7.30.

Inhaltsperzeichnis. Einleitung. Erster Abschnitt. Die Inetrumente und Beobachtungsmethodeu. 1. Die Ortsbestimmung: Sternbilder. Sternnamen. Sternkoordinaten. Präzession. Relativer Ort. Verwandlung der Koordinaten. 2. Vie Helligkeit: Visuelle Helligkentbbestimunung. Die photometrische skala. Die l'hotometer und ihre Theorien. Photographische Sterngröken. Extinktion des Lichtes in der Atmosphäre und in Welteuraume. 3. Wie Farbe der Gestime. Die Schnidische Skala. PurkinjePhänomen. 4. Las Spektrum, Vosels Sternklasscn. Secchis, Pickeringe, Lockyers Klassiłzierung. 5. Die Eutfernung: Wirkung auf den Ort der Gestirne. Absolute und relative Messung. Photographische Methode. Doppelsterne. Relative und absolute Parallaxe. 6. Die Bewegung: I ie Higenbewegung. Die Radialgeschwinaigkeit. Die toiale Bewegung. 7. Die Steruverteilumg: Die schcinbare Verteilung. Zusanmenhang zwischen Steruzahl, Helligkeit und Entiernuag. Anweudung der Walırscheinlichkeitslehre. - Zneiter Abschnitt. Die Eiuzelresultate. 1. Der Steruort: Sternkataloge. Sterukarten. Die Durchmusterungen. Die photogra phische Himmelskarte. 2. Die Helligkeit. Angaben des Ptolemüus. Schätzungen Argelanders und Goulds. Die photometrischen Messungen und ihre Vercleiching. Sterngröbe nach deu photographischen Aufuahmen. Photometrische Grölie der Sonne. 3. Lie Sterufarbe: Osthoffs Katalog. Potsdamer Katalog. Einfluh der Kärbung auf die Helligkeitsuessung. 4. Das Spektrum: Spektroskopische Durchmusterungen. Verteilung der Spektra. Verteilung der Sterne der einzelnen Spekiralklassen. 5. Die Entfernung: Die Einzelresultate. Sterne mit großer Parallaxe. Zusammenhang zwischen der Luffermung und der absolnten Helingeit, bzw, dem spektrum. 6. Die Bewegungen: Kataloge der Ligenbewegungen. Werte der ladialgeschwindigkeiten. Eriblïrung der Bewegungen. Herschels Arbeiten. Bessels Mlethode. Die Grundgleichumgen zur Bestinmung der Sonnembewegung. Argelanders, Airys Methodo. Kapteyns Bestimmung. Größo der Sounenbewegung nach diesen Hethoden. Die Resultate aus den beobachteten Radialgeschwindigkeiten. Resultato aus den totalen Bewegungen. Unzulänclichkeit der Darstelluug und ihre Ursachen. Nichtgeradlinge ungleichlörmige Bewegung. Systematische Hehler der Eigenbewegungen. Beziehungen der Bewegungen zur Milchstraßo. Schoenfelds Mothode und deren Resultate. Bakhuyzens Untersuchungen. Koholds noue Untersichungen nach der Bessel- Koboldscheu Methode und ihre Resultate. Gogenüberstellung. Gesetzmäligkeiten in den Eigenbewegungen. Engere sternsysteme. Beziehungen zwisclien der Bewegung und der Helligkeit bzw. dem Spektraltypus. 7. Die scheinbare Verteilung der Sterne und ihre Beziehung zur Milchstrabe: Herschels Eichungen. Struves Zählungen. Littrows Bearbeitung der B. D. Houzeaus Zählung. Goulds Kreis. Schiaparellis und Stratonoffs Arbeiten. Pickerings Untersuchungen. Seeligers Darstellung der Sternzahlen. - Dritter Abschnit. Der Bau des Fixsternsystems. 1. Das Phänomen der Milchstrabe: Die statistischen Resultate. Seeligers, Plassmanus, Eastons, itratonoffs und Risteuparti graphische Darstellungen. Charakter und Struktur der Milchstralie. Lage der Milchstraße. 2. Die räumliche Anordnung des Universums: Herschels sterusystem. W. Struves Theorio. Darstellunz der Sternzahlen durch dieselbe. Wert für den Extiaktionskoeffizienten. Schiaparellis Aunahmeu. Seeligers Lösung. Abhängigkeit der Entfernung von der Helligkeit und der (Bröße der Eigenbewegung. Gyldøns und Kapteyns Ausdrücke für die mittlere Parallaxe. Comstocks Untersuchungen an sehr schwachen Sternen. 3. Die Bewegungen im Universum: Argelanders und Mädlers Hypothese. Bcobachtungsresultate in Globularsystemen. Mathematische Varstellung, Untersuchung einzelner Spezialsysteme. - Schlutwort. - A n hang. 1. Tafel der Sterne mit bekannter Parallaxe. 2. Tafel der Sterne mit grober Nigenbewegung. 3. Literaturverzeichnis. Register.

Verlag von Friedr. Vieweg \& Sohn in Braunschwelg 


\section{Die Fortschritte \\ der kinetischen Gastheorie}

Von Dr. G. Jäger, Professor der Physik an der techn. Hochschule in Wien. Mit 8 Abbildungen. XI, $121 \mathrm{~S}$. 1906. Geh. M 3.50, geb. M 4.10.

Inhaltsverzeichnis. Einleitung: Grundriß der kinetischen Gastheorie. 1. BoyleCharlessclies Gesetz. 2. Avogadros und Gay-Lussacs Regel. 3. Ialtens Gosetz. 4. Zahlenwert der Gesehwindigkeit. 5. Maxwells Gesetz. 6. Mittlero Weglängo und Stobzahl der Molokeln. 7. Spezifische Wärme. 8. Innere Reibung. 9. Wärmeleitung. 20. Diffusion. 11. Grëße der Molekoln. 12. A bweichungen vom Boyle-Charlesschen Gesetz, 13. Das Virial. - I. Boltzmanns $H$-Theorem. II. Maxwell-Beltzmanusches Gesetz. II. Gultigkeit des Maxwell-Boltzmannselien Gesetzes fur beliebig kleine Kraftelder. IV. Die Zustandsgleichung schwach komprimierter Gase. V. Der Temperaturkeeffizient der jnneren Reibung. VI. Ver 'lemperatursprung bei dor Wirmeleitung. VII. Die ideale Flussigkeit. VIII. Innorer Druck der Flüssigkeiten. IX. Innere Reibung idealer Flüssigheiten und Grëße der Molekeln.

\section{Aus den Besprechungen.}

Chemiker-Zeitung. ${ }_{n}$ Die ausführliche Einleitung des Werkchens gibt eine ausgezeichnete klare Darstellung der kinetischen Gastheorie. Schon wegen derselben kann das Büchlein, das aus der Feder des durch scine ${ }_{n}$ theoretische

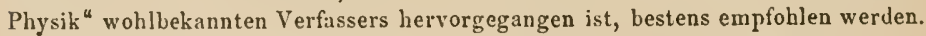
Der Hauptteil ist zunächst Boltzmanns Untersuchungeu gewidmet. Das H-Thcorem und seine Beziehung zum zweiten Hauptsatzo der Wärmetheoris finden zucrst ihre Ableitung, sodann die Sätze über Geschwindigkeitsverteilung und Dichteverteilung in eiuem Gase, in dem innere und änßere Kräfte wirken. Der Verf. verfolgt hier anschauliche und originelle Methoden. Die Anwendung wird auf die Zustandsgleichung nicht zu stark komprimierter Gase gemacht. wobei der Verf, den Arbeiten vou Ml. Reinganum 1olgt. Der 'Temperaturkoeffizient der inneren Reibung, der in letzter Zeit befriedigende Erklärung fand, wird ebenfalls besprochen. Es folgen die Untersuchungen von Smolnchowski über den Temperatursprung der Wärmeleitung in Gasen und eigene Forsehuugen des Verf. über die Theorie der Flüssigkeiten. Das Büchlein kann daher allen, die sich für die auch in der Elektrizitätslehre immer melır Bcdeutung gewinnende kinetische Theorie interessieren, wärmstens empfohlen werden."

Elektrochemische Zeitschrift. "Mit Bezug aut die Wichtigkeit, die gegeuwärtig die Arbeiten über die Elektrizität in Giasen erlangt haben, dürfte die vorliegende kurze und dabei doch in bezug auf die Hauptmomente erschöpfende Zusammeufassung der Resultate der kinetischen Gastheorie nicht unwillkommen sein. Die Darstellung ist eine klare und deutliche und es ist fast durchweg eine eingehende mathematische Begrïndung gegeben." 


\title{
Petrogenesis
}

\author{
Von Dr. C. Doelter, o. Frofessor der Mineralogie und \\ Petrographie an der Universität Graz. Mit einer Lichtdruck- \\ tafel und 5 Abbildungen. XII, 262 S. 1906. Geh. \\ M 7.-, geb. M 7.80.
}

\begin{abstract}
Inhaltsverzeichnis. Einleitung. - Erstes Kapitel. Das Erdinnexe und der Vulkanismug. Vulkanische Herde. Peripherische Vulkanherde. Ursachen des Aufdringens des Magmas. Eruptionsfäligkeit des Msgmas. Verhalten des vulkanischøn Magmas boim Erstarren. Verhalten der Gase. Temperatur der Lava. Temperatur der Vulkanherde. - Zweites Kapitel. Die Erscheinungsformen der vulkanischen Gesteine. Die vulkanischen Gesteine. Einflub des Druckes auf die Bildung ron Tiefeugesteinen. Die Rolle der Mineralisatoren. Das Auftreten der Gesteine. Eruptionsformen der Oberflächengesteine. Viskesität und Lagerunggform. Ergcheinungsformen der Tiefengesteine. Mechanismus der Intrusion. Der äußere Habitus der vulksnischen Gesteino. - Drittes Kapitel. Die Struktur der Eruptivgestein $\theta$. Struktur der Effusivgesteine. Struktur der Tiefengesteine. Speziello Strukturen. Bezieliungen zwischen dem Alter der Gesteine und ihrer Struktur. Änderungen in der Struktur und dem Mineralbestande in verschiedenen Teilen einer Eruptionsmasse. Viertes Kapitel. Abhängigkeit dor mineralogischon Zusammensetzung der Gestein $\theta$ von ihrom chemischen Bestande. Dissoziation des Magmas. Vergleich der Gesteinsmagmen. Graphische Darstellung von Gesteinsmagmen, - F ü $\mathrm{n}$ tes Kapitel. Die Differentiation der Magmen. Das Ganggetolge. Die Hypethese Bröggers. Differentiation bei künstlichen Schmelzen. Magmstische Differentiation durch das spezifische Gowicht. Verhalten fertiger Kristallo im Magma. Dio Kristallisatiousdifferentiation. Schlieren. Differentiation bei gleichbleibender chemischer Zusammensetzung. Umschmelzungsversuche von Mineralien und Gesteinen. - Sech s tes $\mathrm{K}$ a pitel. Die Altersfolgo der hruptivgesteine. Unterschiedo der Altersfolge bei 'Tiefonund Effusivgesteinen. Veränderungen dex vulkanischen Produkte im Laufo geolegischer Perioden. Petrographische Charakteristik und Altersbeziehungen der Gesteine eines Vulkans, - Siebentes Kapitel. Die Ein s chlüs seder Gesteine. Exogene Einschlusso. Endogene (hemöogene) Einschlüsse. Die Olivinknollen. - A chtes Kapitel. A s similation und Korrosion. Ursache der Korrosionen und Resorptionen. Korrosion des Nebengesteins am Kontakt. Assimilation. - Nountes Kapitel. Künstliche Gesteine. - Zehntes Kapitel. Die Verfestigungdes vulkanischen Magmas. Dio Ausscheidungsfolge dor Mineralien im Magma. Kristallisationsvermögen und Kristalligationsgeschwindigkeit. Unterkithlung. Einfluß der Schmel zpunkte. Das Kristallisationsmikroskop. Einfluß des Iruckes auf die Ausscheidung. Bildung vulkanischer Tuffe, Elftes hapitel. Dio Kontaktmetamorphose. Kaustische Wirkungen. Umwandluug von Kalksteinen. Umwandlung von Sandsteinen, Tonschiefern und 'Toueu. Chemische Vorgänge bei der Phyllitkontaktmetamerphose. Proumatelytische Metamorphose. Umwandlung des Diabases und der Diabastuffe. Chemisch-physikalische Vorgänge bei der Kontaktmetamorphese. - Zwölftes Kapitel. Die Bildung der kristallinen Schiefer. Allgemeines. Exuptive Gneise. Gneise als umgewandelte Granite. Diagenege. Uar Regionalmetamorphismus. Die chemische Zusammensetzung der kristallinen Schiefer. Umwandlung durch Waser. Umwandlung durch holie Temperatur. Die Injektiensliypethese. Die Dynamometamorphese. Chemische Reaktion im Festen. Die Plastizitat der Gesteine. Einfluß des Druckes auf die Lỏslichkcit von Mineralien. Einseitiger Druck (Streß, Pressung). Zusammenhang der Motamorphose nit der Dislokstion. Das Velumgesetz. Der Mineralbestand der kristallinen Schiefer. Struktur und Textur der Schiefergosteine. Ursache der Schiefrigkeit. Die Tiefenstufcu. Schwierigkeiten einer allgemeinen Anwendung der Dynamemctamorphese. Bildung ven kristallinen Schiefern durch Kontaktmetamorphose. Vergleich der Kontaktmetamorphose und der Dynamomotamorphose. - Dreizehntes Kapitel. Sedimente. Kalksteine. Dolomit. Magnesit. Kieselsinter, Kiøsclschiefer. Saudsteine. Tone, Kaolin. Äolischo Sedimente, Alaungchiefer. Laterit. - Vierzehntes Kapitel. Chemigche Absätze, Bildung von Steinsalz, Gips und Anhydrit. Absätzo der Salzseen. Die Barrentheorie. Gips und Anhydrit. Steinsalz und Abraumsalze. Reihenfolge der Ablagerungen der Salzminerslien. Dio Temperatur der Steinsalzlager. Einfiu $B$ der Zeit und des Druckes. Salpoter. Soda. - Nachträge. - Autorenregistex. - Sachregister.
\end{abstract}




\title{
Die Wissenschaft
}

\section{Die Grundlagen der Farbenphotographie}

\author{
Von Dr. B. Donath. Mit 35 Abbildungen und \\ einer farbigen Ausschlagtafel. VIII, 166 S. 1906. \\ Geh. M 5.-, geb. M 5.80.
}

Inhaltsverzeichnis. I. Teil. Die direkten Verfahren der photographischen Farbenwiedergabe. Farbenwiedergabe, Krstes Kapitel. Die photographische Farbenwiedergabe durch stehende Lichtwellen. Geschichtliohes. 'Theorio des Verfahrens: Begriff des Wellenstrahles. Lichtwellen. Reflexion der Lichtwellen (Phasenverlust). Schoinfarben durch Interfereuz. Die Zenkersche Theorie. Experimentelle Beweise fur die Richtigkeit der Theorie (Veränderung der Farben mit dem Beobachtungswinkel und durch Auseinandertreten der Elemeutarspiegel. Komplementäre Farben im durchfalleuden Lichte. Nachweis der Elementarschichten in mikroskopischen Dtinnschnitteu). Weitere theoretische Betrachtungen (Die Beziehungen

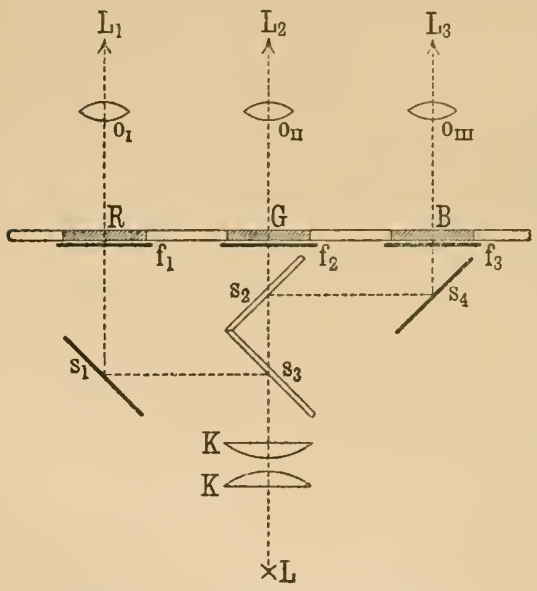
des Silberkornes zur Schichtenbildung. Die speziellen optischen Eigenschafteu von Chromgelatine, kohärentem u. molekularem Silber. Elementarspiegelabstand und Phasenverlust. Abhängigkeit der Farhenwiedergabe von der Expositionszeit. Die Beziehungen der Tiefenwallo zur Oberfiachenwelle. Lippmanacho Spektra hoherer Ordnung). Praktische Ausubung des Lippmannschen Verfahrens. - Zweites Kapitel. Die photographioche Farbenwiedergabe durch Korperfarben. Geschichtliches. Theorio des Verfahrens. Ausübung des Ausbleichverfahrens. II. Teil. Die indirekten Verfahren der photographischen Farbenwiedergabe. Erates Kapitel. Geochichte nnd Theorie des Drei. farbenverfahren 8 . Geschichtliches. Theorie: Additive u. subtraktive Farbenmischung. Geometrische Konstruktion der Mischfarben. Grundfarben. Die Theorien der Farbenwahrnebmung ron Young - Helmholtz und Hering. Experimentelle Bestimmung der Grundfarben. $\mathrm{Zweites}$ Is a itel. Die photographischo Analyso nach den drei Grundfarben. Sensibilisatoren und Filter: Die Bcziehungen der Aufuahmefilter zu den Reproduktionsfiltern und Sensibilisatoren. Die praktische Lurchfübrung der Analye: Die Sensibilisierung der Platte. Aufnahme und Entwickelung. Einflußs der Schwärzungskurve auf die Richtigkeit der Farbenwiedergabe. - Dritte $\mathrm{K}$ apitel. Die additipeSynthese der Teilbilder (Grenzen der authentischen Reproduktion). - Viertes Kapitel. Additive Wieder. gabomit Hilfe von Beugungsopektren (Theorie und Ausübung des Verfahrens). - Funftes Kapitel. Additive Farbenwiedergabe mit dem Dreifarben: raster. - Seehster Kapitel. Die oubtraktive Synthese der Teilbilder. Theorie: Wahl des Farbensystems. Beziehungen zwischen dem Grundfarbensystem, den Aufnahmefiltern und Sensibilisatoren. Ausfuhrung der subtraktiven Synthese: Die Herstellung transparenter Dreifarbenbilder. Subtraktive Bilder auf reflektiereader Grundlage. Der Dreifarbendruck (Blachdruck und Hochdruck), - Literaturverzeichniø, Namenverzeichnis. 


\title{
Höhlenkunde
}

\section{mit Berücksichtigung der Karstphänomene}

\author{
Von Dr. phil. Walter von Knebel. Mit $42 A b$ - \\ bildungen im Text und auf 4 Tafeln. XVI, 222 S. \\ 1906. Geh. M 5.50, geb. M 6.30.
}

Inhaltsverzeichnis. 1. Kapitel. Einführung. 2. Kapitel. Die Ursachen der E.ohlenbildung. 3. Ka pitel. Die Verteilung der Hohlen in den Gesteinarten der Erdrinde. 4. K \& pitel. Verkarstung und Karstphänomene. Б. Kapitel. Grundwasser uud Quellen in Hohlengebieten. 6. Kapitel. Die Korrosion in Karstgebirgen. 7. Kapitel. Die mechrnische Tätigkeit des Wassers in bezug auf die Hohlenbildung. 8. Kapitel. Morphologie der Hohlen; natürliches System der Hoblenformen. 9. Kapitel. Hölenflusse. 10. Kapitel. Die Vaucluse und die Vauclusequellen. 11. Ka pitel. Die Grundwassertheorie zur Erklärung der hydrographischen Probleme des Karstes. 12. Ka pitel. Submarine Quellen und Meeresschwinden slg Beweise für das Vorhandensein von Höhlentlüssen. 13. Ka pitel. Die Entstehung von Hoblenfiussen. 14. Kapitel. Dolinen. 15. Kapitel. Bedeutung der Dollnen für die Intstehung von Talern. 16. Kapitel. Kesseltăler. 17. K a pitel. Die wichtigsten Höhlengebiete. 18. Kapitel. Halbhöhlen. 19. Kapitel. Ursprüngliche Höhlen. 20. Kapitel. Meteorologische Verhälnigse in Hohlen. 21. Ka pitel. Die biologischen Verhältnisse in Hölen. 22. K a pitel. Höhlen als Wohnorte der prähistorischen Menschen. 23. Kapitel. Kulturarbeit in Höhlengebieten. Geschichte der Hoblenkunde.

\section{Aus den Besprechungen.}

Geologisches Zentralblatt. ${ }_{n}$ Verf. bat in diesem Buche die Ergebnisse jahrelanger Studien in verschiedenen Höhlengebieten Deutschlands und im Karst unter Berücksichtigung der umfangreichen Literatur niedergelegt und so ein wissenschaftliches Buch über den Gegenstand verfaßt, über den bisher nur Einzelbeschreibungen vorhanden waren. Gleichzeitig gibt er bestimmte Hinweise und Anleitungen $z u$ gewissenhaften Beobachtungen anf diesem interessanten Gebiet geologisch-geographischer Forschung. Die einschlägige Literatur wird erwähnt und kritisch behandelt..."

Zeitschrift für die österreichischen Gymnasien. $n$... Der Verfasser hat es verstanden, die einschlägigen Erscheinungen nicht nur in sehr lichtvoller Weise zu beschreiben, sondern such deren Entstehung in sachgemäßer Weise zu begründen. Die verschiedenen Anschauungen werden gegeneinander abgewogen und in vollkommen objektiver Weise beurteilt. Besonderes Interesse ist im vorliegenden Buche dem Karstphänomene entgegengebracht worden und dies in Anbetracht der Wichtigkeit der Kenntnis dieser Erscheinung für die Bodenkultur mit vollem Rechte. Es findet auch die kulturelle Verwertung verkarsteter Länder die entsprechende Erörterung."

Mitteilungen der Geographischen Gesellschaft für Thüringen. „... Der Verfasser gibt eine erwünschte Gesamtdarstellung der Höhlenkunde und aller damit zusammenhängenden Fragen. Jeder, der sich schnell orientieren will üler ein besonderes Kapitel dieser Wissenschaft, wird gern vorliegendes Buch zur Hand nehmen. Die zahlreichen Abbildungen, die dem Werke beigegeben sind, sind recht instruktiv und klar und schließen sich der sonstigen Ansstationg des Buches würdig an."

Verlag von Friedr. Vieweg \& Sohn in Braunschwelg 


\title{
Die Wissenschaft Sammung naturvissenschatht und Heft 16
}

\section{Die Eiszeit}

\author{
von Dr. F. E. Geinitz, o. Professor an der Universität \\ Rostock. Mit 25 Abbilo'ungen, 3 farbigen Tafeln \\ und 1 Tabelle. XIV, 198 S. Geh. M7.-, geb. M7.80.
}

Inhaltsverzeichnis. Einfithrung. Fauna und Flora des Quartärs. Gletscherentwickelung in Quartär. Verbroitung des quartären Glazialphänomens. Frühere Eiszeiten. Landverteilung vor der Eiszeit. Ursache der Fiszeit. Zeitberochnungen. Die Glazialablagerungen. Einfluß der Vereisung auf den Untergruud. - I. Das nordouropäische Glazial. 1. Gebiet Skandinavien-Rußland-Norddeutschland-Holland. a) Art des Vorkommens und Verbreltung: Skaudinaviev. Fiunland. Rufland. Bornholm. Dänemark. Norddeutschland. Holland. b) Gliederung des nordeuropaischen Quartärs: Präglazial (Altquartär); Fluvioglazial oder Extraglazial. Interglazial. c) Die Verhältnisse nach dem Abschmelzen der Eisdecke (Postglazial, Spätglazial). d) Die postglazialen Niveauschwankungen. 2. Das Glazial Großbritannieng, - II Das Glazialphänomen der Alpen. - III, Das Gebietzwischen alpiner und nordischer Vergletacherung. 1. Die extraglazialen Ablagerungen, ihre Gliederung und Beziohung zum prähistorischen Menschen. 2. Die vergletscherten deutschen Mittelgebirge und ihr Vorland. - IV. Eiszeitgletecher im ubrigen Europa. - V. DieEiszeit Nordamerikas. - VI. Die Polarlunder. - VII. Die Eiszeit a uf den ubrigen Kontinenten. Asien. Afrika. Sudamerika. Australien. Antarktik. Grahamland.

\section{Aus den Besprechungen.}

Zeitschrift für Schulgeographie. „Der bekannte Mecklenburger Forscher auf dem Gebiete der Glazialgeologie bat hier ein Kompendium seines Forschungsgebietes gegeben, wie es knapper und zutreffender kaum gegeben werden konnte. Der Test ist eng zusammengedrängt, nicht gerade leicht zu lesen, erteilt aber dafür über alles, was mit der Fiszeit irgendwie in Beziehung steht, genaue und zuverlässige Auskunft. Man mag sich ïber die Moorfrage mit Bezug auf Ḱlimaschwankungen oder über die Niveauschwankungen des Baltikums orientieren wollen, alle diese Erscheinungen cbarakterisiert Geinitz in kurzen treffenden Worten. Das fehlende Register wird durch das eingehende Inhaltsverzeichnis genügend ersetzt, so daB sich das Werk auch zum Nachschlagen sehr eignet..."

Blätter für das bayerische Gymnasialschulwesen, „Der Verfasser gibt an der Hand der neueren Forschungen einen recht anschaulichen Überblick iber unser gegenwärtiges Wissen von diesem vielumstrittenen Zeitraum der Erdgescbichte. Daber dürfte dieses Buch, das zum Teil ein Auszug aus seiner größeren Arbeit über das Quartär Nordeuropas ist, besonders dem Geographen willkommen sein; denn dieser Stoff ist in solcher Abrundung mit stetem Hinweis auf die einschlägigen Fragen und literarischen Hilfsmittel meines Wissens sonst nirgends zu finden." 


\title{
Die Wissenschaft
}

\section{Die Anwendung der Interferenzen in der Spektroskopie und Metrologie}

\author{
Von Dr. E. Gehrcke, Privatdozent an der Universität \\ Berlin, technischer Hilfsarbeiter an der physık-techn. Reichs- \\ anstalt. Mit 73 Abbildungen. IX, 160 S. 1906. \\ Geh. M 5.50, geb. M 6.20.
}

Inhaltsverzeichnis. I Teil: Allgemeine Einleitung. 1. Wellenbewegung. 2. Lichtwellen. 3. Funktion der Linsen, 4. Das Auge als optischer Apparat. 5. Fernrohr und Mikroskop. 6. Helligkeit der durch Linsen erzengten Bilảer. 7. Wellenlänge, Fortpflanzungsgeschwindigkeit, Sohwingungsdauer. 8. Sinuswellen. 9. Prinzip der Superposition. - II. Teil: Erzengung und Theorie einiger augewählter Interferenzerscheinungen. 10. Fresnels Spiegelversuch. 11. Interferenzen an planparallelen Platten. 12. Interferenzen an keilförmigen Platten. 13. Fresnels Biprisma, Newtons Farbenglas, Michelsons Interferomeier. 14. Überlagerung der Interferenzen verschiedener Wellenlängen. 15. Die Quecksilberlampe. 16. Intensitätsverteilung der Interferenzen an planparallelen Platten. 17. Berücksichtigung der vielfach reflektierten Strahlen. 18. Weitere Jiskuøsion der berechneten Intensitätsverteilung. 19. Intensitätsverteilung der Interferenzen im reflektierten Lichte. 20. Planparallele Luftplatte zwischen zwei rechtwinkeligen Glasprismen. 21. Vorhandensein zweier komplementärer Interferenzsystem $\theta$ im reflektierten Licht. 22. Beugung des Lichtes an einer Öfinung. 23. Beuguug an mehreren (Bpaltförmigen) öffnungen. - IIT. Teil: Spektral a parate. 24. Fizeaus Modifikation des Newtonschen Farbenglases. 25. Ausbildung der Fizeauschen Methole durch Nichelson. 26. Fraunhofers Beugungsgitter. 27. Reflexionsgitter. 28. Interferometer von Yerot und Fabry. Lummers Doppelkeil. 29. Míchelsons Stufengitter. 30. Interferenzspektroskop von Lummer und Gehrcke. 31. Allgemeine Theorie aller auf der Erzeugung vou Interferenzstreifen beruhender Spektralapparate. 32. Abhängigkeit der Intensitätsverteilung der Interferenzen von der Breite des Kollimatorspaltes. 23. Auflüsungsvermögen u. Dispersionsgebiet. 34. Interferenzpunkte. 35. „Falsche" Spelstrallinien und ihre Erkennung mit Hilfo der Interfereuzpunkte. 36. Auflösungsvermögen des Prismas. 37. Finfluf der Leugung an der Offnung einer Linse anf die von ihr entworfenen Bilder. Grenze der Auflösung im Fernrohr und Mikroskop. 38. EinfluB der Beugung auf die Sichtbarkeit der Interferenzen an keilförmigen und planparallelen Platten. - IV. Teil: Auswahl von Resultated der spektrookopischen Forschung überden Mechanismus des Leuchtens. 39. Trabanten. 40. Dopplerвches Primzip. ${ }_{n} B r e i t e^{4}$ der Śpektrallinien. 41. Abhängigkeit der Breite der Spektrallinien von der Temperatur, dem Molekulargewicht und der Erregungsart. 42. Der Stark-Effekt. 43. Einfluc des Druckes auf die Wellenlänge. 44. Der Zeeman-Effekt. 45. Theorie des Zeeman-Effektes, 46. Anomaler Zeeman-Effekt. Dissymmetrie in schwachen Feldern. 47. Interferenzfähigkeit de日 Lichtes einzelner Spektrallinien. 48. Serien. - V. Teil: Anwendungen der Interferenzen zu physikalischen Meв в ungen und in der Metrologie. 49. Bestimmung von Variationen der optisehen Dicke sogen. planparalleler Platten. 50. Anwendungen der Interferenzen zu verschiedenen physikalischen Messungen. 51. Anwendungen der Interferenzen in der Astronomie. 52. Interferentialrefraktor von Jamin. 53. Modifikationen von Michelsons Interferometer. 64. Lichtwellen als Längeneinheiten. 55, Michelsons Auswertung des Meters in Lichtwellen. 56. Methode von Benoit zur Bestimmung der Ordnungszahl von Interferenzen. 57. Methode von Perot und Fabry zur Bestimmung der Ordnungszahl von Interferenzen. 58. Einheit der Masse. 59. Methode von Macé de Lépinay zur Messung der Dicke und des Brechungsexponenten planparalleler Platten. 60. Wellenlägennornalen. 61. Interferenzen planparalleler Platten im kontinuierlichen Spektrum. Literaturverzeichnis. Register. 


\title{
Kinematik organischer Gelenke
}

\author{
Von Prof. Dr. Otto Fischer in Leipzig. Mit \\ 77 Abbildungen. XII, 261 S. 1907. Geh. M 8.-, \\ geb. M9.-.
}

Inhaltsverzeichnis. Einleitung. 2. Teil: Uberdie Formen der Gelenkflächen und die ans denselbon ioh ergobonden moglichen Arton der Gelenkbewegungen. A. Gelenke mit ausgedehutem Flächenkontakt. 1. Gelenke mit starren Flächen. 2. Gelenko mit deformierbaren Flächen. B. Gelenke mit geringem Flächenkontakt. 3. Allgemeine Betrachtungen uber die Arten dor Gelenkbowegungen. 4. Zylindergelenke. 5. Gelenke mit beliebiger Form der Gelenkflächen. 6. Die Winkelgeschwindigkeiten der versohledenen Komponenten der allgemeinsten Gelenkbewegung. 7. Einflub der Deformierbarkeit des Gelenkknorpals auf die Bewegung in Geleuken mit gerlugem Flächenkontakt. 8. Ausfüllang der Gelenkspalten. - 2. Teil: Über die Bewegungsfreibeit. 9. Die Bewegungsfreiheit in einzelnen Gelenken. 10. Die Bewegungsfreiheit in Gelenksystemen. - 3. Toil: Bewegung in epeziellen Gelenken. 11. Allgemeine uber die Methoden der Untersuchung epezieller Gelenke. 12. Timpiriache Ableitung spezieller Bewegungen eines ganzen Gelenksyetems. 13. Spezielle Beispiele bestimmter zu einem Geleuk gehörender Relativbowegungen. 14. Bewegungsgesetze in speziellen Geleuken von zwei Graden der Freiheit. Lehrbüclier, in denen organische Gelenke behandelt werden. Monographien über Gelenke und Gelenkbeweguug. Sachregister.

\section{Aus den Besprechungen.}

Deutsche Literaturzeitung. „Das ganze Buch ist mit beneidenswerter Präzision und Prägnanz geschrieben, der Aufwand an Geometrie und Algebra ist $s 0$ bescheiden, daß man erwarten sollte, Mediziner und Zoologen werden sich durcharbeiten können. Jedenfalls wird es für sie die beste Einführung in die Geleukmechanik sein. Für alle aber, die das Studium der Gelenke als Spezialität betreiben, wird die Fischersche Kinematik ganz unentbehrlich sein."

Leipziger Medizinische Monatsschrift. „...Wenn Fischer, der durch seine Forschungen auf diesem Gebiete längst bekanut ist, auch in bescheidener Weise sagt, daß sein Werk kein Lelırbuch der in den lebenden Körpern vorkommenden speziellen Gelenke sein soll, so müssen wir es doch als ein solches ansehen, denn er hat es verstanden, uns in klarer und übersichtlicher Weise die Verhältnisse, auf die es ankommt, darzulegen. Wir haben die meisten Werke, die sich mit dem Gegenstande der Gelenklehre oder der Statik and Mechanik des menschlichen linochengerüstes beschäftigen, in der Hand gehabt, müssen aber sagen, daß uns kejnes eine derartige präzise Auskunft und klare Vorstellung der Verluältnisse gegebea hat wie die Kinematik Fischers..."

Reichs-Medizinal-Anzeiger. „Ie! Aufgabe der organischen lKinematik, die kinematischen Gesetze besonders deu Zoologen, den Medizinern und den gebildeten Laien klar zu machen, hat Verf, sich in dem vorliegenden Buche unterzogen und diese Aufgabe vorzüglich gelöst..." 


\title{
Franz Meumann und
}

\section{sein Wirken als Forscher und Lehrer}

\author{
Von Dr. A. Wangerin, Professor an der Universltät \\ Halle a. S. Mit einer Textíigur und einem Bildnis \\ Neumanns in Heliogravüre. X, 185 S. 1907. Geh. \\ M5.50, geb. M 6.20.
}

Inhaltsverzeichnis. Frster Teil: Franz Neumanns Leben. - Zweiter Te1l: Neumanns wissenschaftliche Arbeiten. 1. Die kristallographisch-mineralogischen Arbeiten. 2. Arbeiten zur Wärmelehre. 3. Arbeiten aus der Optik und Elas tiziti.tatheorie. 4. Arbeiten uber induzierte Ströme. 5. Mathematische Arbeiten. 6. Wissensehaftliche Untersuchungen Neumanns, die nicht von ihm selbst veröffentlicht gind. - Dritter Tell: Vorlesungen, Seminar, Laboratorium. 1. Die gedruckten Vorlesungen, 2. Das Seminar. 3. Neumanns Bestrebungen zur Errichtung oines physikalischen Laboratoriums.

\section{Aus den Besprechungen.}

Die Physikalische Zeitschrift schreibt: ${ }_{n}$ Die Materie des letzterschienenen Heftes der Sammlung Die Wissenschafte bildet die Biographie eines großen Gelehrten, des Königsberger Physikers und Mathematikers Franz Neumann. Nicht mit Flittern äußeren Glanzes umgibt A. Wangerin die markante Persönlichkeit dieses Mannes, für dessen edle Bescheidenheit und berzgewinnende Güte er aber nicht genug Worte finden kann. In schlichten einfachen Worten schildert der Verfasser die harten Entricklungsjahre mit ihren zahlreichen Entbebrungen, die der Lebrer und Forscher Neumann durchzumachen hatte, um sich dann eingehend mit Neumanns wissenschaftlichen Arbeiten zu befassen. Neq man $\mathrm{ns}$ erste Arbeiten liegen auf kristallographisch-mineralogischem Gebiet. Später aind es Beiträge zur Wärmelehre, Optik und Elastizitätstheorie. Aus der Elektrizitätslehre bearbeitete er die induzierten Ströme. Seine bedeutendste mathematische Arbeit ist diejeuige über Kugelfunktionen. Das Buch enthält ferner Mitteilungen über Arbeiten aus Neumanns Seminar und Laboratorium.

Nicht ohne ein guwisses Mitleid wird man das letzte Kapitel lesen, welches von Neumanns Bestrebungen zur Errichtung eines physikalischen Laboratoriuns berichtet..."

Deutsche Literaturzeitung. „... Einer der ältesten überlebenden Schüler Fr. Neumanns, Prof. A. Wangerin in Halle, hat sich der Aufgabe unterzogen, Fr. Neumann als Forscher und Lehrer zu schildern, und nicht nur die anderen Schüler des großen und trefflichen Mannes, zu denen auch der Ref. sich zählt, alle Physiker sind dem Verfusser dafür zu Dank verpflichtet, $\mathrm{da} B$ er mit solcher Hingabe und mit solcher Beherrschung des Stoffes seine Aufgabe gelöst hat. Auch bezüglich der Beurteilung der verschiedenen Neumannschen Leistungen kann sich der Ref, in allen wesentlichen Punkten dem Vert. völlig anschließen..." 


\title{
Die Zustandsgleichung der Gase und Flüssigkeiten und die Kontinuitätstheorie
}

\author{
Von Prot. Dr. J. P. Kuenen in Leiden. Mit \\ 9 Abbildungen. X, 241 S. 1907. Geh. M 6.50, \\ geb. M 7.10 .
}

In ha It sverzeichnis. 1. Kapitel. Kondensationserscheinungen und Kontinuitatsprinzlp. 2. Ka pitel. Kinetische Theorie idealer Gase. 3. Ka pitel. Kinetische Theorie unvollkommener Gase: Zustandsgleichung. 4. Ka pitel. Erklärung der Verflussigungserscheinungen nach der Zustandagleichung; Erweiterung der Kontinuitätatheorie. 5. Kapitol. Anormale Kondensations- und kritische Erscheinungen: A. Nichtkonstanz des Dampfdruckes, B. Kritische Erscheinungen. 6. bis 9. Kapitel. Vergleioh der Zustandsgleichung mit der Erfahrung: A. Kritische Gleichungen. B. Homogene Zustände. C. Sättigungsgebiet. D. Thermische Größen. 10. Kapitel. Molekulare Dimensionen. 11. Kapitel. Gesetz der korrespondierenden Zustände. Gleichformigkeitsprinzip. 12. und 18. Kapitel. Verbesserung der Zustandsgleichung; Anzuwendende Merkmale: A. Theorie der Volumkorrektion. B. Theorie der inolekularen Attraktion: Verbesserung der beiden Korrektionsglieder. 14. Kapitel. Mathematische Methoden der Herleitung der Zustandsgleichung.

\section{Aus den Besprechungen.}

Physikal. - chemisches Zentralblatt. „Der Verf. hat eine schwierige Aufgabe übernommen, in Form einer Monographie das im Titel bezeichnete Thema $z u$ bearbeiten. Sicher vielen wird das vorliegende Buch willkommen sein.

Die wohlgeordnete Zusammenfassung des Bekannten und die objektive und kritische Behandlungsweise machen es einerseits dem Fachmanne wertvoll, der eine Fülle von Anregungen zur weiteren theoretisch-mathematischen oder experimentellen Ausgestaltung des Probiems finden wird. Besonders das bis jetzt zutage geförderte experimentelle Material ist absolut unzulänglich, hior har't noch ein großes, fruchtbares, aber auch äußerst schwioriges Gebiet der eingehendeu experimentellen Bearbeitung.

Anderseits sind einzelne Kapitel allgemeineren Inhalts so einfach und anregend geschriebelı, daß diese vereint auch dem Anfänger mit mäßigen Kenntnissen in der höheren Mathematik ein abgerundetes Bild über das Wesen und die Erfolge der Zustandsgleichung and der sich ihr anschließenden Fragen geben können.

Lobend sei noch der sorgfältigen Literaturangaben gedacht und deren zweckmäßigen systematischen Zusanmenstellung am Schlusse jedes größeren Kapitels."

Jahrbuch der Chemie. „... Dic Darstellung der vorliegenden Monographie ist musterguiltig und setzt, was vielen Chemikern besonders erwünscht sein dürfte, kein allzu großes $M a ß$ mathematischer und theoretisch-physikalischer Kenntnisse voraus."

Verlag von Friedr. Vleweg do Soln in Braunschweig 


\title{
Radioaktive Umwandlungen
}

\author{
Von E. Rutherford, Professor der Physik an der \\ Mc Gill-Universität in Montreai, Übersetzt von M. Levin. \\ Mit 53 Abbildungen. VIII, 285 S. 1907. Geh. M8.- \\ geb. M 8.60 .
}

Inhaltsverzeichnis. The Silliman Foundation. - Preface. - Vorbemerkung zur deutschen Ausgabe. - Kapitel 1. Historische Einleitung. - Kapitel 2. Die radioaktiven Umwandlungen d. Thoriums. - Ka pitel 3. Die Radiumemanation. - Ka pitel 4. Die Unwandlungen des aktiven Niederschlages des Radiums. - Kapitel 5 . Der langsam sich umwandelnde aktive Niederschlag des Radiums. - Kapitel 6. Ursprung und Lebensdauer des Radiums, - Kapitel 7. Die Umwandlungsprodukte des Uranium und Aktiniums und der Zusammenhang zwischen den Radioelementen. - Kapitel 8. Die Entstehong ron Helium aus Radium und die Umwandlung der Materie. - Ka pi tel 9. Die Radioaktivität der Erde und der Atmosphäre. - Kapitel 10. Die Eigenschaften der a-Strahlen. - Kapitel 11. Radioaktivo Prozesse im Lichte physikalischer Anschauringen.

\section{Aus den Besprechungen.}

Zeitschrift für den physikalischen und chemischen Unterricht. „... Im März 1905 hat $R$ utherford an der Yale University eine Reihe von Vorlesungen gehalten, die hauptsächlich des Verf. eigenstes Arbeitsgebiet, die radioaktiven Umwandlungen, zum Gegenstand hatten. In der vorliegenden Veröffentlichung sind indessen alle bis zum Beginn von 1907 erschienenen Arbeiten berücksichtigt. Dem Buch haftet noch in der Übersetzung etwas ron der lebhaften Frische des Vortrages an. Meisterhaft in ihrer klaren Knappheit ist die historische Einleitung, die uns zeigt, wie in ca. 10 Jahren durch das Handinhandarbeiten von Physikern und Chemikern aller Länder unsere Kenntnisse von Materie und Strahlung erweitert und vertieft worden sind. Den kühnsten Schritt tat eben Rutherford in der Aufstellung der Umwandlungshypothese, die alle bisherigen Anschaungen von Elementen und Atomen umstieß, aber eine Fülle von Erscheinungen zusammenfaßte, die vorher nur verwirrten. Die Hypotliese reicht noch jetzt ans, um alle seit ihrer Anfstellung gefundenen Tatsachen zu erklären, und dennoch - zum Lobe des Autors sei es besonders hervorgehoben - weiß Rutherford scharf zwischen Beobachtung und Spekulation, zwischen der Tatsache und ihrer vermutlichen Erklärung zu unterscheiden. Jedes Wort der Empfehlung ist bei diesem Buche überAliissig."

Literarisches Zentralblatt. $\#$... Die Vorträge sind in anregeuder und so anschøulicher Form niedergeschrieben, daB Physiker und Nichtfachmann, sofern er über einige caturwissenschaftliche Bildung verfügt, Genuß und Gewinn durch aie Lektüre haben wird. Für den Fachmann ist das Heft eine Quelle anregender Gedanken und Anschauungen, während der Laie eine gute Einführung in unsere gegenwärtige Kenntnis der Radioaktivität vorfindet, neben welcher die Atomzerfallstheorie, die Elektronentheorie zur Erörterung gelangt und die Bedeutung für unser Wissen von der Luftelektrizität sowie für dell Ausbau und die Bestätigung wichtiger physikalischer Grundanschauungen gebührend geltend gemacht wird."

\section{Verlag von Friedr. Vieweg \& Sohn in Braunschweig}




\title{
Kant und die Maturwissenschaft
}

\author{
Von Prof. Dr. Edm. König in Sondershausen. \\ VI, 232 S. 1907. Geh. M 6.-, geb. M 7.-.
}

Inhaltsverzeichnis. 1. Kapitel. Naturwisensehaft und Natnrphllosophie. - 2. Kapitel. Kaut nnd die Naturwissenschaft seinerZeit. 1. Eiufluß der Naturwlssenschaft auf Kants Philosophie. 2. Kant als Naturforscher. 3. Kapitel. Die Leitsätge der kritischen Erkenntnislehre. 1. Die kritische Faseung des Erkenntnisproblems, 2. Anschauung und Denken - Aposteriori und Apriori. 3. Der Raum. 4. Die Denkformen (Kategorien). 5. Grenzen der Erkenntnis - Endergebnisse. - 4. Kapitel. Kants Einwirkung af die Naturwissenschaft des 19. Jahrhundert8. - 5, Kapitel. Das Problem des Raumes und der Be. wegung. 1. Der Anschauungsraum. 2. Der Raum der Geometrie. 3. Der physische Raum. - 6. Kapitel. Erschoinung und Wesen - Erfahrung und Theorie (Kritik des Phänomenalismus). - 7. Kapitel. Das physikalische Problem. 1. Die Grundlagen der mechanischen Naturanschauung. 2. Die Prinzipien der Mechanik. 3. Die Konstitution der Materie. 4. Kinetik und Energetik, - 8. Ka pitel. Das biologische und das psychophysiche Problem. 1. Gegensatz der mechanistischen und der teleologischen Biologie. 2. Der Zweckbegriff bei Kant. 3. Ist der Zweckbegriff Kategorie? 4. Die Hauptformen der naturwisaenschaftlichen Teleologie. ธ. Die psychophysische Kausalitst. 6. Schluß, Zusätzo.

\section{Aus den Besprechungen.}

Zeitschrift des Vereins deutscher Ingenieure. „Der Verfasser sucht zu zeigen, daß insbesondere die erkenntnistheoretischen Anschauungen Kants, denen sich die Erkenntnistheorie der neneren Naturwissenschaft in verschiedenen wesentlichen Punkten in bemerkenswerter Weise ganz von selbst genähert hat, mit den Ergebnissen der naturwissenschaftlichen Forschung durchaus vereinbar und geeignet sind, als Grundlage für eine einheitliche Lösung der naturphilosophischen Probleme zu dienen. Das Bnch wird allen denen willkommen sein, die sich allgemein über die Hauptströmungen in der beutigen Naturphilosophie unterrichten möchten. Die Ingenieure werden die Kapitel über Raum und Bewegung, über die Grundlsgen der mechanischen Naturanschauung, über die Prinzipien der Mechanik und über Kinetik und Energetik besonders interessieren. "

Chemiker-Zeitung (am Schluß einer langen Besprechung). „... Im Rahmen einer Besprechung, selbst einer (mit Rücksicht auf die Schwierigkeit des Gegenstandes) schon ungewöhnlich langen, kann natürlich weder auf Einzelheiten eingegangen, noch mit dem Verfasser über deren Auffassung und seinen Gesamtstandpunkt gerechtet werden; doch dürften schon obige Andeutungen genügen, um die Leser dieser Zeitschrift auf die Fülle wichtiger Lehren und Gedanken hinzuweisen, die das Königśche Buch enthält, und die namentlich den Naturforscher anregen sollten, auch seinerseits weiter zu denken und, unbeirrt durch jegliche Autorität, nach fernerer Aufklärung zu streben. ,Aufgeklärt seinc, so sagt. Kant, sheißt: den Mut haben, sich seines eigenen Verstandes zu bedienen. 


\title{
Synthetisch-organische Chemie der Neuzeit
}

\author{
von Dr. Julius Schmidt, a. o. Professol an der
}

Königl. Technischen Hochschule in Stuttgart. X, $185 \mathrm{~S}$. 1908. Geh. M 5.50, geb. M 6.20.

Inhaltsverzeichnis. Einleitung. Krlanterung des Begriffes Synthese. Historsche Bemerkungen. Über die Behandlungaw $\theta i s e$ des Stoffes. Kohlensuboxyd. Kaallsäure. - 1. Kapitel. Bedeutung der Organomagnesiumhaloide fur synthetische Zwecke. 3. Kapitel. Einige synthetiache Ergebnisse aus der Zuckergruppe. Asymmetrische Synthese, - 3. Kapitel. Synthetische Realstionen, welche zu Aldehyden uad Ketonen füren. - 4. Kapitel. Dimethylsulfat als Mlethylierungsmittel. - 5. Kapitel. Synthesen mit Hilfe von Aziden. - 6. Ka a itel. Methoden von W. Fischer zur Synthese von Polypeptiden. - 7. Kapitel. Synthesen durch Aufapaltung und Umwandlungen zyklischer Basen. - 8. Kapitel. Synthesen auf dem Gebiete der Alkaloidchemie, der künstlichen Arzneimittel und in der Puringruppe. - 9. Kapitel. Synthesen von Farbstoffen und mehrkernigen aromatischen Verbindungen. - 10. Kapite1. Synthesen von Riechstoffen, von hydroaromatischen und diesen pahestehenden Verbindungen. - 11. Ka pitel. Synthesen verschiedener organischer Verbindungen auf elektrochemischem Wege - Namenregister. - Sachregister.

\section{Aus den Besprechungen.}

Literarisches Zentralblatt. „Das 23. Heft der, Wissenschafte bildet eine höchst willkommene Ergänzung unserer Lehrbücher der organischen Chemie in mehrfacher Hinsicht. Enthält es doch neben den kurz angedeuteten üblichen Synthesen in ausführlicher Besprechung neuere Verfahren, welche in den Lehrbüchern nicht oder höchstens ganz obertlächlich gestreift werden, so namentlich die vielseitige Anwendung der Organomagnesiumhaloide, stets unter eingehender Würdigung des wirtschaftlichen Wertes der betreffenden Methode. $\mathrm{Da}$ die Darstellungsverfahren der Duftstoffe, Farbstoffe nnd Heilmittel ebenfalls in den Rahmen der Besprechung fallen und das Buch bei aller wissenschaftlichen Strenge doch leicht faßlich geschrieben ist, so kann es unbedenklich nicht nur dem Fachmann, sondern auch weiteren Kreisen (Pharmazeuten, Physioloyen, Ärzten usw.) nachdrücklich empfohlen werden."

Zentralblatt für Pharmazie und Chemie. "Die synthetisch-organische Chemie hat in der Neuzeit, d. h. in den letzten 10 bis 15 Jabren Errungenschaften aufzuweisen von so allgemeinem Interesse, wie sie sich nie hatten voraussehen lassen. Die vorliegendo Schrift soll ein Bild derselben entwerfen. In ihr sind die außerordentlich zahlreichen Ergebnisse je nach ibrer größeren oder geringeren Bedeutung mehr oder weniger ausführlich behandelt worden. Dabei hat der Verfasser mit Rücksicht auf den größeren Leserkreis, für den das Buch bestimmt ist, für eine leicht faßliche, aber doch streng wissenschaftliche Form des meist aus den Quellen geschöpften Materials Sorge getragen und auch die Wichtigkeit einschlägiger Entdeckungen in wirtschaftlicher Hinsicht entsprechend gewürdigt.

Gerade das vorliegende Thema mit seinen mannigfachen Beziehungen zum jrakíitchen Leben dürfte verhältnismäßig leichter als manch anderes abstrakteres Gebiet der Naturwissenschuften das Interesse eines weiteren Kreises fesseln..." 


\title{
Die \\ chemische Affinität und ihre Messung
}

\author{
Von Dr. Otto Sackur, Privatdozent an der Universität \\ Breslau. Mit 5 Abbildungen. VIII, 130 S. 1908. Geh. \\ M 4.-, geb. M 4.80.
}

Inhaltsverzeichnis. 1. Kapitel. Die historische Entwickelung des Affinitätsbegriffes. Ältere Anschauungen ther die chemische Verwandtschaftskraft. Die Abhängigkeit der Affinität von der Menge der sich umsetzenden Stoffe. Die Aviditat der Săuren und Basen. Quantitative Messung der Affinitit in mechanisohem Maße. Definition der Affinität als maximale Arbeit nach van 't Hoff. - 2. Kapitel. Der Begriff der maximalen Arbeit und der zweite Hauptsatz der Thermodynamik. Da. T'homson-Berthelotsche Prinzip. Der erste Hauptsatz. Der zweite Hauptsatz. Die naximale Leistung einer Arbeitsmaschine, Der Carnotsche Kreisprozeß. Die Arbeitsleistung chemischer Vorgänge. Die Helmholtzsche Gleichung. - 3. Kapitel. Die Berechnung der Affinitat aus dem Betrageder Umsetzung. I. Reaktionen im homogenen System, a) Zwischen Gasen: Thermodynamische Ableitung des Massenwirkungsgesetzes; Experimentelle Bestimmnng von Gasgleichgewichten (Statische Methoden. Dynamisohe Methoden). b) Reaktionen in Losungen: Die Avidität von Säuren und Basen. II. Reaktionen im heterogenen System. a) Zwischen festen Stoffen und Gasen: Experimentelle Method on zur Bestimmung d. Dissoziationsspannung; Berechnung der Affinităt der Metalle zam Sauerstoff und den Halogenen. b) Reaktionen zwischen festen Stoffen und Lösungen. c) Affinitat zwischen festen Stoffen. - 4. Kapitel. Elektrische Methode der Affinitătsmessung. Die maximale Arbeit eines galvanischen Elementes. Ketten vom Typus des Daniellelementcs. Konzentrationsketten. Affinitat der Komplexbildung. Das absolute Potential. Gasketten. Oxydationg- und Reduktionsketten. - 5. Kapitel. Affinität und Temperatur. Die Gleichung der Reaktionsisochore. Berechnung der Affinität aus der Wärmetönung. Die Affinität in der Năbe des Umwandlungspunktes. Änderung der Wärmetönung mit der Temperatur. Die Nernstsche Theorie zur Berechnung von Gleichgewichten aus thermischen GröBen. 6. Kapitel. Ergebuisse der Affinitätsmessung. Reaktionen zwischen Verbindungen. Reaktionen zwischen den Elementen, - Schlußbetrachtung.

\section{Aus den Besprechungen.}

Chemiker-Zeitung. „Die Aufgabe, die sich der Verfasser in der vorliegenden Monographie gestellt hat, deu großen Fortschritt, den die Chemie der thermodynamischen Betrachtungsweise verdankt, anschaulich darzustellen, hat er in sachgemäßer Weise und Form gelöst. Das Bucl übermittelt trotz seiner kurzeu Fassung die wesentlichsten Errungenschaften der chemisch verwerteten Thermodynamik prägnant und zuverlässig, so daß es dem engeren und weiteren Kreise der Fachgenossen Belehrung und Anregung gibt. ... Das Buch ist jedem $z u$ empfehlen, der eine nicht an der Oberfläche haftende Kenntnis des Gegenstandes in großen Zügen sich aneignen will, zumal dem Studierenden als Ergänzung und Unterstützung bei thermodynamischen Vorlesungen." 


\title{
Die Korpuskulartheorie der Materie
}

\author{
Von Dr. J. J. Thomson, Mitgl. der Royal Society, \\ Professor der Experimentalphysik an des Unlversität in \\ Cambridge und Professor der Physik an der Royal Institution \\ in London. Autoris. Übersetzung von G. Siebert. \\ Mit 29 Abbildungen. VIII, 166 S. 1908. Geh. M5.- \\ geb. M 5.80 .
}

Inhaltsverzeichuis. 1. Kapitel. Elnleitung. Korpuskeln in Vakuumröhren. 2. Kapitel. Der Ursprung der Masse der Korpuskel. 3. Ka pitel. Eigenschaften einer Korpuskel. 4. Kapitel. Korpuskulartheorie der Leitung in Metallen. 5. Kapitel. Die zweite Theorje der elektrischen Leitung. 6. Kapitel. Die Anordnung der Korpuskeln Im Atom. 7. Kapitel. Uber die Anzahl der Korpuskeln im Atom. - Register.

\section{Aus den Besprechungen.}

Naturwissenschaftliche Wochenschrift. In dem vorliegenden Werke legt der Verfasser, der den Physikern als einer der geistreichsten Forscher auf dem Gebiete der Elektronik wohl bekannt ist, seine Anschauungen über den Aufbau der Materie in ziemlich populärer Form dar. Das Buch ist also als eine Fortsetzung und Erweiterung der im Jalıre 1904 ebenfalls dentsch in der Sammlung Die Wissenschaft (Heft 3), erschienenen Vorträge ,Elektrizität und Materie anznsehen.

In der neuen Schrift werden zunäcbst die grundlegenden Tatsachen der Elektronentheorie besprochen. Daran schließt sich ein Kapitel, in dem die Frage nach dem Ursprung der Masse der Elektronen mit dem Ergebnis diskutiert wird, daß die Masse der Elektronen nur scheinbar materiell, in Wahrheit aber elektromagnetischer Natur sei. Eingehend wird die Korpuskulartheorie der Wärme- und der Elektrizitätsleitung in Metallen behandelt und gezeigt, daß von den beiden konkurrierenden Theorien die eine, nach der die die Leitung der Wärme und Elektrizität besorgenden Elektronen insofern danernd im Metall frei sind, als sie mit den Atomen ihrer Umgebung, von denen sie sich durch Dissoziation getrennt habeu, in einer Art von Temperaturgleichgewicht stehen, zu einem Widerspruch mit der Erfahrung führt, indem der Wert für die spezifische Wärme der Metalle, wenn sie richtig wäre, viel größer (bei Silber zehnmal so groß) sein müßte, als er tatsäcblich ist. Die andere Theorie, welche voraussetzt, daß die Elektronen nicht dauernd, sondern nur während der kurzen Zeit frei sind, die sie zur Zurücllegung des Weges von einem Atom zum Nachbaratom brauchen, vermeidet diese Schwierigkeit, und ihr ist, da sie alle anderen Beobachtungen ebensogut wie die erste Theorie erklärt, der Vorrang zu geben. Zwei Kapitel ïber den Autban der chemischen Atome aus positiver Elektrizität und negativen Elektronen und deren Anordnung im Atom beschließen das Buch...

Die Lektüre der SKorpuskulartheorie der Materie ist nicht leicht, aber sie bietet dem, der die Mühe der Durcharbeitung nicht scheut, einen großen Genuß." 


\title{
Die Bindung des atmosphärischen Stickstoffs in Matur und Technik
}

\author{
Von Dr. P. Vageler in Königsberg i. Pr. Mit \\ 16 Abbildungen im Text und auf 5 Tafeln. VIII, \\ 132 S. 1908. Geh. M 4.50, geb. M 5.20.
}

InhaItsverzeichnis. I. Einleltang. - II. Die Hanptquellen des gebundezen stickstofis. - III. Bindung von Stickstoff ohne Mitwirkung von Organismen. - IV. Die Bindung von atmosphärischem Stickstoff durch frei lebende Bakterien. 1. Grundlagen und Vorarbeiten. 2. Clostridium pasteurianum Win, und Verwaudte. 3. Die Azotobakter gruppe und sonstige stickstoffsammelnde Bakterien. 4. Stickstofiblndung auf künstlichen Nahrbøden. - V. Die Stickstofibindung durch frei lebende Bakterien im Boden. VI. Stickstoffbindung durch constige frei lebende Organismen. - VII. Stickstoffbindung durch Bakterien und soustige Mikroorganismen im Verein (Symbiose) mit grunen Pflanzen. 1. Grundlagen und Vorarbeiten. 2. Die Zuchtung der Knöllchenerreger auf kunstlichem Nährboden. 3. Knollchenbakterien und Wirtspflanzen. - VUI. Dio Bodenimplung mit Knöllchenbakterien. - IX, Leguminosen als Stickstoffeammler in der Praxis. - X. Die Blndung des atmosphärischen Stickstoffs in der Technik. 1. Die Gewinnung des Luftstickstoffe mit Hilfe der Elektrizitä, 2. Kalkstickstofi und Stickstoffkalk. - Scblußbetrachtung. - Register.

\section{Aus den Besprechungen.}

Monatsblätter des wissenschaftlichen Clubs in Wien. "Drohende Erschöpfung der Salpeterfundstätten, zunehmende Verwendung stickstoffhaltiger Däugmittel in der Landwirtschaft, anwachsender Bedarf von Salpetersäuro in der chemischen Industrie machen es zu einem der wichtigsten Probleme der Hand in Hand arbeitenden technischen und Naturwissenschaften, sioh in der Herstellung stickstoflhaltender und stickstoffabgebender Substanzen ron dem gebundenen Stickstoff unabhängig und das unendliche Stickstoffreservoir der atmosphärischen Luft der Menschheit nutzbar zu machen.

Soviel man weiß, wird Stickstoff in der Natur von gewissen Pflanzen assimiliert unter Vermittlung lebender Organismen, lösliche Stickstoffverbindungen bilden sich in geringer Menge in der Luft, aber die fortgeschrittene Technik unserer Zeit hat eiu Verfahren gefunden, den fast reaktionsunfähigen trägen Stickstoff der Luft durch Überleiten über erhitzte Karbide technisch zu verwerten und als jüngstes Glied in der Kette epocbaler Erfolge der Elektrochemie den Stickstoff der Luft durch Durchleiten in elektrischen Flammenbogen zu oxydieren und solann in lösliche Salze überzuführen.

Das hübsch illustrierte Büchlein, das das 26. Heft der im Viewegschen Verlage erscheinenden Sammlung , Die Wissenschafte bildet, legt das Hauptgewicht der Darstellung auf die Assimilation des Stickstofies durch lebende Organismen, welcher Abteil ungefähr die Hälfte der Seitenzahl umfaßt. Die Darstellung ist klar, ungemein populär und gleichzeitig wissenschaftlich, für die Interessenten der Frage, als da sind: Chemiker, Techniker, Landwirte, Volkswirtschaftler und Biologen ist es bestens zu empfehlen." 


\title{
Die Schwerebestimmung an der Erdoberfläche
}

\author{
Von Prof. Dr. Joh. Bapt. Messerschmitt, \\ Konservator des Erdmagnetischen Observatoriums und del \\ Erdbebenhauptstation in München. Mit 25 Abbildungen. \\ VIII, 158 S. 1908. Geh. M 5.-, geb. M 5.80.
}

In haltsverzeichnis. I. Allgemeine Begriffe. 1. Richtung der Schwere. 2. Schwerpunkt. 3. Maß der Schwerkraft. - II. Der freie F'all. 1. Gesetze. 2. Die Schwere wirkt auf alle Körper gleich. 8. Fallmaschinen. - II. Allgemeine Schwere oder Gravitation. - IV. Das Pendel. 1. Mathematisches Pendel. 2. Physisches Pendel. 3. Spezielle Fälle. V. Bestimmung der Intensitat der Schwere durch Pendelmessungen. - VI. Absolute Messungen der Schwerkraft. 1. Fadenpendel, 2. Reversionspendel, - VII. Relative Schwerebestimmung. 1. Sternecksche Pendel. 2. Apparat von Defforges. 3. Andere Apparate. - VII. Reduktion auf Meereshöhe. - IX. Die normale Schwerkraft in Meereshöhe. - X. Verteilung der Schwere auf der Erde, Konstitution der Erdrinde, - XI. Räumliche und zeitliche Verk̉nderung der Schwerkraft, Krümmungsverhaltnisse der NiveauAăchen. - XII. Einfluß der Schwerkraft auf die geometrischen Höheumessungen. Literatur. - Register.

\section{Aus den Besprechungen.}

Zeitschrift der Gesellschaft für Erdkunde. "Trotz der großen Bedeutang, welche die Schwerebestimmungen an der Erdoberfläche besonders in den drei letzten Jahrzehnten infolge der Vervollkommnung der Beobachtungs-Hilfsmittel and Methoden gewonnen haben, ist doch die Theorie und Praxis der Messungen in weiten Kreisen so gut wie unbekannt geblieben. Hieran war zum Teil wohl Schuld, dab sich, so weit dem Referenten bekannt ist, in der vorhandenen Literatur kein geeignetes Buch für eine eingehendere, dabei aber keine grðßeren Ansprüche an die mathematische Vorbildung des Lesers stellende Einführung in das Gebiet der Schweremessungen findet. Diesem Mangel hilft das vorliegende Buch in glücklicher Weise ab..." 


\section{Die Kraftfelder}

Von V. Bjerlines, Plofessor der Mechanik und del maihematischen Physik an der Universität Christiania. Mit 29 Abbildungen. XVI, 174 S. 1909. Geh. M 7.-, geb. M 7.80 .

Inhaltsverzeichnis. Finleitung. 1. Kapitel. Kinetischer Auftrieb. 2. Kapitel. Die Kraft gegen einen pulsierenden Körper. 3. Kapitel. Felder und Fernwirkungen pulsierender Körper. 4. Kapitel. Flussigkeitsbewegungen, erzeugt durch die Impulse außerer Kräfte. 5. Ka pitel. Felder und scheinbare Fernwirkungen oszillierender Korper, 6. Ka pitel. Grenzflächenbedingungen an Heterogenitätsstellen. 7. Ka pitel. Heterogenes flussiges System mit undurchdringlichen Kórpern. 8. Ka pitei. Umriß der Theorie der Vektorfelder. 9. Kapitel. Utber schwingende Bewegungen. 10. Kapitel. Die Grundgleichungen des hydrodynamischen Feldes mit undurchdringHchen Körpern. 11. Kapitel. Hydrodynamische Kraftfelder mit durchströmten Körpern. 12. Kapitel. Kraftfelder in flussigen Medien mit gyrostatisehen Eigenschaften.

\section{Aus den Besprechungen.}

Annalen der Elektrotechnik. "Seit der Zeit Newtons pflegten die Physiker ihren Erklärungen der physikalischen Erscheinungen durchgehends die Vorstellung ron Fernwirkungen zugrunde zu legen. Erst Faraday stellte dieser Auffassung die Idee des Kraftfeldes gegenïber. Nach ihm legte Maxwell in seinen berihmten Gleichungen die formalen Beziehungen des elektromagnetischen Kraftfeldes zu Raum und Zeit fest. Diese Theorie Maswells erhielt durch die klassisclien Versuche von H. Hertz eine glänzende Bestätigung, die den Erfolg hatte, daß von jetzt ab die Vorstellung von Kraftfeldern die Fernwirkungshypothese vollständig verdrängte. Was uns die Maxwellsche Theorie in endgültiger Form gegeben hat, ist aber nur die formale Bezieliung der elektrischen und magnetischen Vektorgrößen zu Raum und Zeit. Über die innere Natur der Kiraftfelder weiß man auch durch sie nichts Näheres. Das hier vorliegende Buch enthält in übersichtlicher Zusammenstellung die Resultate einer langen Reihe von Forschungen, welche unternommen sind init dem Ziele, womöglich Licht auf diese dunkle Frage zu werfen. Unmittelbarer Gegenstand der Untersuchung sind nicht die elektromagnetischen Kraftfelder selbst, sondern ihnen analoge Felder, die in bewegten Flüssigkeiten und in Medien mit gewissen Elastizitätseigenschaften auftreten. Für das Studium dieser Felder hat der Verfasser neue Methoden geschaifen, welche eine einfache Ableitung der früher schwer zugänglichen Resultate gestatten. In sehr einfacher Weise entwickelt er die Theorie zweier Klassen von bydrodynamischen Felderscheinungen, der C. A. Bjerknesschen, wo schwingende, und der Euler-Kelvinschen, wo stationäre Bewegung der Flüssigkeit zugrunde liegt. Die bekannte, in beiden Fällen auftretende Analogie mit elektrostatischen oder magnetischen Feldern wird eingehend dargelegt, und die zur Verifikatiou der Resultate dienenden Versuche werden beschrieben. Als unmittelbare Fortsetzung dieser hydrodynamischen Untèrsuchung entwickelt der Verfasser die Theorie ähnlieher Kraftfelderscheinungen in Medien mit Elastizität der eigentümlichen gyrostatischen Art, welche $\mathrm{Mac} \mathrm{Cullagh}$ zur Erklärung optischer, und Lord Kelvin zur Veranschaulichung elektrodynamischer Erscheinungen einführten nnd die nach ihnen viele Forscher benutzt haben, um mechanische Bilder der allgemeinsten elektromagnetischen Felderscheinungen zu konstruieren." 


\title{
Physiologie der Stimme und Sprache
}

\author{
Von Prof. Dr. Hermann Gutzmann in Berlin. \\ Mit 92 zum Teil farbigen Abbildungen im Text \\ und auf 2 Tafeln. X, 208 S. 1909. Geh. M 8.- \\ geb. M9.-
}

Inhaltsverzeichnis. I. Phy6iologieder Atmung und Stimme. A. Ana. tomische Vorbemerkungen. 1. Atmung. 2. Stimme. B. Die Atembewegungen beins Sprechen. 1. Registrierung der Atembewegungon. 2. Typus der normalen Sprechatmung. Hegistrierung des A temvolumeus beim Sprechen. C. Die Stimme. 1. Bilduny der stimme. 2. Tonhöhe und Tonstärke. 3. Register. 4. Genauigkeit der Stimme. 5. Stimmeinsätze. 6. Stimmlage und Stimmumfang. 7. H'lusterstimme u. Bauchuednerstimme. - II. P hy siologie der Sprachlaute. A. Anatomische Vorbemerkungen. Der Aufbau des Ausatzrohres. B. Die Physiologie des Ansatzrohres: 1. Klanganalyse der Sprachlaute: a) Analyse der menschlichen Stimme durch einfaches Hören. U) Graphische Aualyee der Klänge. c) Klangkurven. d) Analyso der Klangkurven. e) Resultate der Klanganalyse. f) Theorie der Vokale. g) Synthese der Vokale. h) Analyse der Konsonanten. 2. Formen und Bewegungen des Ansatzrohres: a) Analyse der Sprachbewegungen durch Beobachten. b) Analyse der Sprachbewegungen durch registrierende Instrumente: Akustische legistrierung; Optische Kegistrierung; Direkte McLmethoden, c) Anwendung der liegistrierung auf die eiuzelnen Bewegungen: Luftbewegung der Artikulation; Kehlkopfbewegungen: Unterkieferbewegungen; Zunge und Mundbodeu; Gaumensegel; Lippen. d) Apparate fur die Gesamtaufuahme der Artikulationsorgane. e) Wirbemelhoden. 3. Nie sprachlaute: a) Vokale und Konsonanten. b) Die Vokale. c) Die Kousonanten: Verschluflaute, Mledia nd Tenuis; Reibelaute; $L$-Laute; $R$-Laute; Resonanten; Laute des vierten Artiknlationssystems; Kehlkopflaute; Sthualilaute (Clixe). d) Die Sprachlaute in der Verbiudung: Doppelvokale und Doppelkonsonanten; silbe, Wort, Satz, 4. Wie Akzente der Sprache. б. Die phonetische schrift. - Literaturverzcichuis.

\section{Aus den Besprechungen.}

Berliner klinische Wochenschrift. „Wie sehr Verfasser den Gegenstand beherrsclat, geht aus der Klarheit seiner Darstellungen hervor; die Schilderung der an sich oft recht schwierigen Forscliungsinethoden und die Deutung der Resultate lält dem Leser manclies einfach und rerständlich erscheinen, was in Wirklichkeit nur durch mühsame Arbeit klargelegt werden konnte. Das Literaturverzeichnis enthält 263 Nummern. Das Buch ist unentbelırlich für den Spracharzt, den Laryngologen und für den Physiologen, ferner für den Taubstummenlelırer, tür deu Gesaıgspädagogen und den Lelıer der Rlıetorik; aber auch für die Philologen, Linguisten und Phonetiker enthält es viel Wissenswertes. Vielleicht bekehrt es auch den einen oder andereu jener rückständigen Philologen, welche die experimentelle Plonetik noch nicht als Wissenschaftszweig anerkeunen wollen. Für jeden Arzt aber wird die Lektiire des Buches, dem wir ein glänzendes Prognostikon stellen, belehrend uud genußreich sein."

Miedizinische Klinik. $n$... Durch seine eingehende, durch zahlreiche eigene Spezialforschungen begründete Orientierung auf diesem Wissensgebiet ist in der Tat Gutzmann in hervorragender Weise beruten und befäligt, die Kernpunkte zu erkennen und herauszuheben und eine wirklich gute Darstellung dieser für den Arzt und den Psychologen, Physiologen und den Physiker gleich wichtigen Materie zu geben. Das Buch kann der Beachtung derjenigen, welehe in diesem und den angrenzenden wissenscliaftlichen Gebieten arbeiten, nur warm empfohlen werden." 


\title{
Die atmosphärische Elektrizität
}

\section{Methoden und Ergebnisse der modernen luftelektrischen Forschung}

\author{
Von H. Mache, a. o. Prof. a. d. Techn. Hochschule in Wien, \\ und E. v. Schweidler, a. o. Prof. a. d.Unversität in Wien. \\ Mit 20 Abbildungen. XI, 247 S. 1909. Geh. \\ M 6.-, geb. M 6.80.
}

\begin{abstract}
Inhaltsverzeichnis. 1. Kapitel. Daselektrische Feldder Atmosphrare. Allgemeine Eigenschatten des Feldes. Instrumentarium zur Messung des Potentialgefáltes. Methude der Messung des Potentialgefalles am Eruboden. Reduktion auf die Ẻbene. Methode der Messung des Potentialgefälles im Ballon. Beobachtungsresultate. - 2. Kapitel. Die Elektrizitatsleituug der Atmosphäre. Coulombs Zorstreuungsgesetz. Der Llster-Geltelsche Zerstreuungsapparat. Grundzüge der Ioneutheorie. Anwendung der Ionentbeorie auf die Apparate zur Bestimmung der Leitung dor froien Atmosph 2. Der Elstex-Geitelsche Zerstreuungsapparat ohne Schutzzylinder. 3. Scherings Zerstreuungsapparat. 4. Gerdiens Aspirator. Beobachtungsresultate: a) Zerstreuungsbeobachtungen mit Schutzzylinder; b) Zerstreuungsmessungen mit freistebendem Zerstreuungskörper; c) Absolute Messungen des Leitvermögens, - 3. Ka pitel. Wie Ionen dor A tmosphare. Ionemzalı, Luerts Aspirator. Ionenbeweglichkeit. Wiedervereinigung der Ionen (Molisierul.g). Adsorptiou und Dlffusion der Ionen. - 4. Ka pitel. Itie Ionisatoren und Hiektrisatoren der Atmogphäre. A. Hlektrisierung und Ionisierung beim Zerspritzen von Wasser in Luft. B. Elektrlsierung durch die Emigsion ron Elektronen von belichteteu Ubertïchenteilen der Erde. Licbtelektrische Aktinomotrie. C. Ionisieruug durch ultraviolettes Licht. D. Ionisiorung durcb Becquerelstrahlung: a) Wie radioaktiven substanzen; b) Das Vorkommen radioaktiver Substanzen auf der Erde und in der A tmosphäre: I. Allgemeine Verbreitung radioaktiver Substauzes im K'rlboden; Radioaktivität von Gesteins- und Erdartən; Radioaktivitiat der Bodenluft; Radioaktivitüt der Wuellen. II. Vorkommen radioaktiver Emauatiouen und deren Zerfalisprodukte in der Atmosphäre. III. Die Bedeutung der radioaktiven Substanzez fur die Ionisation der Atmosphäre. - 5. Kapitel. Elektrische Strómungen in der Atmosphäre, A. Wer normale vertikale Leitungsstrom. B. Der durch die Zerfallsprodukte des Radiums und Thoriums getragene Strom. C. Konvektionsströme durch Luftboweguug. D. Konvektionsströme durch Niederschläge; Wilsons Kondensations. theorie K. Summatiou der elektrischen Vertikalströme in der Atwosphäre. - 6. K a pitel. Lenchtende Eutladungen in der Atmosphäre. A. Elektrische Gasentladungen im allgemeinen. B. Leuchtende Entladungen bei Gewitteru: 1. Die Entladungsformen bei Gewittern. Ir. Iuneurheoretische Einorduung der naturlicben Eutladungen. UI. Die meteorologischen Bedingungen des Lintstehens leuchtender sutladungen. C. Das Polarlicht. - 7. Kapitel. Theorien der atmosphäriscben Elektrizität. - Literaturnachweis.
\end{abstract}

\section{Aus den Besprechungen.}

Elektrotechnische Zeitschrift. ,In knapper, aber sehr klarer Form wird in diesem Buche geschildert, welcher Methoden sich die jetzige luftelektrische Forschung bedient, um die elektrischen Vorgänge in der Atmosphäre zu untersuchen, welche Ergebnisse allgemeinen Charakters dabei gewonneu wurden und welche Voraussetzungen theoretischer Natur sich als heuristisch wertvoll erwiesen haben..." 


\title{
Das Klimaproblem der geologischen Vergangenheit $u$. histor. Gegenwart
}

\author{
Von Dr. Wilh. R. Eckardt, Assistent am meteorolog. \\ Observatorium und der öfentl. Wetterdienststelle Aachen. \\ Mit 18 Abbildungen und 4 Karten. XI, 183 S. 1909. \\ Geh. M 6.50, geb. M 7.10.
}

Inhaltsverzeichnis. Das Klimaproblem der geologiвchen Vergangenheit und historischen Gegenwart. Einleitung: ' $Z$ weck und Bedentung des Gegenstandes. Das Verbälinis der Geographie zu den Naturwissenschaften, insbesondere zur Geologie. Die Bedeutıng der krgebnisse der geolugischen t'urschung für die Geographie. Lie Klimatologie. - Die Bodenbildung unter dem Liutlusse des Klimas: Die mechanische Zerstörung des festen Gesteins. Die chemische Zersetzung desselben. - 1) a K Kima der geologischen Vergangenheit. - Das Klıwa in Paläozoikum. - Die präkarbonen Perioden. - Das Karbon. - Die permokarbone Eiszeit und die Ǵlossopterıstlora. - Das Klima im Mesozoikun, besonders in der Jura- und Kreideperiodo. - Das Klima In der Tertiärzeit. - Die diluviale Eis- oder Schneezeit. - Die Äuderungeudes Klimas iu bistorischer Zeit, insbesonderedas Austrockungsproblem. - Der Einf́lul des Waldes, bzw. einer Vegetationsdecke auf das Klima und den Wasserabfluß: a) Der Einfluß des Waldes auf die 'iemperaturverhältnisse. b) Der Hinfluli des Waldes auf die Niodersculäge. - Dio Klimaschwankungen; Klima und Wirtschaft. Die allgemeine Koustauz des heutigen Klimas. - Wichtige Aufgaben der Meteorologie und Klimatologie. - Literaturaugaben.

\section{Aus den Besprecliungen.}

Globus. „Der Verfasser hat sich die Aufgabe gestellt, die Klimate der geologischeu Vergangenheit nicht, wie es nach seiner Ansicht seither fast nur geschehen, von rein geologischem Standpunkt, sondern von der allgemein naturwissenschaftlichen Seite zur Darstellung zu bringen. Er kommt dabej zu dem Schluß, daß die gesamten klimatischen Änderungen der geologischen Vorzeit, die in großen Zïgen dargestellt werden, sich aus rein meteorologischen Verhältnissen erklären lassen, die ihrerseits wieder als Folgen von Polverschiebungen infolge geologischer Veränderungen auf der Erde aufgefaßt werden. Eine periodische Wiederkehr von Kältewellen im Permokarbon, Diluvium usw. sowie eine gleichmäßige Temperierung in früher Zeit und erst spätere Differenzierung der Klimate wird abgelehnt und dagegen behauptet, daß schon von früher Zeit her Zonen auf der Eirde bestanden, wenn ihre Unterschiede vielleicht auch zeitweise nicht so ausgeprägt waren wie heute. Der Abschnitt über die Änderungen des Klimas in historischer Zeit behandelt vor allem den Einflus des Waldes auf das Klima bzw. den Zusammenhang dieser beiden. Eine Klimaänderung seit historischen Zeiten ist nach Eckardt nicht nachweisbar. Der Schlußabschnitt weist kurz auf die außerordentliche Wichtigkeit der Erforschung der klimatologischen Bedingungen des PAlanzenwuchses sowie sonstiger klimatologischer Beobachtungen hin. ."

Veriag von Friedr. Vieweg do Soln in Bratnschweig 


\title{
Lichtbiologie. Die experimentellen Grund- lagen der modernen Lichtbehandlung
}

\author{
Zusammengestellt von Dr. med. Albert Jesionek, \\ Professol an der Universität Gießen. VIII, 177 S. 1910. Geh. \\ M 4.-, geb. M 4.80 .
}

Inhaltsverzeichnis. Einleitung. Allgemeine Bemerkungen über das Liclit. - Die Einwirkung des Lichtes auf die PHanzenwelt. Die Liuwirkungen des Lichtes auf die Bakterien. - Die Reızwirkung des Lichtes auf Baklerien und andere Mkroorganismen. -Die photodyuamische Erscheinung. - Einwirkungen des Lichtes auf höhere 'liere. - Einwirkung des Lichtes auf die Haut des Menschen: Sonnenbrand und Gletscherbiand. Erfahrumgen der Polarfahror. Hautentzüudung durch elektrisches Licht. Experimentelle Untersuchungen von Widmarck, Hammer, Finsen. Physiologisches Verlalteu der Haut. Hautröte. Hautpiøment. Horufarbe und Epidermistrubung. Haare. 'liefenwirkung. Penetrationsfäbigkeit der einzelneu Strahlen. Penetratiousfahigikelt der ultraviolctteu Strahlen. Kloiduug. Hitzschlag. - Dio Listologischen Verinderungen im belichteten Hautgewebe. - Das Licht als Ursache von Hautkiankheiten: Lichtentztindung. Schutzmalinahmen. Hydroa aestivalis. Xeroderma pigmeutosum. Sommersprossen, Warzen. Pellagra. Blattern. Kotlichtbeliandlung. Lichtuehaudlung nach Finsen. - Einwirkungen des Lichtes auf das Blut und auf den Stofiwechsel: Lichtregulierung. Quinckes und Behrings Experimente. Lumineszenz des Blutes. - Einwirkuugen des Lichtes auf das Nervensystem: Experimente mit farbigem Licht. ${ }_{n}$ Simnlich-sittliche Wirkung" der Farben uach Goethe.

\section{Aus dem Vorworte.}

$\pi$... In diesem Werkchen beabsichtige ich nun keineswegs vom ärztlichen Standpunkte aus die verschiedenen Arten und Methoden der Lichtbehandlung in allen ihren Einzelheiten zu schildern und meine subjektiven Ansichten iber den Wert und Unwert der verschiedenen lichtherapeutischen Bestrebungen. ausführlich zu erörtern. Der Zweck meiner Ausführungen ist der, irrtünlichen Vorstellungen entgegen zu treten und denjenigeı, die sich für diese Fragen interessieren, zu zeigen, dab sich die inedizinische Forschung mit den Wirkungen des Lichtes auf die beleble Natur aufs eifrigste beschäftigt und bestrebt ist, aus dem Studium des Lichtes und seiner Eigenschaften für den kranken und für den gesunden Menschen möglichst viel Vorteil zu ziehen. Dabei habe ich es mir angelegen sein lassen, aus der reichhaltigen Fülle der Literatur nur diejenigen Arbeiten zusammenzustellen, welche in die Beziehungen des Lichtes zum Leben uns einen sicheren Einblick gewähren und hinsichtlich der praktischen Verwertung des Lichtes zu grundlegenden Ergebnissen geführt haben. Auch mag diese Darstellung dazu dienen, den Leser über die weitausgebreitetell Bahnen und oft verschlungenen Pfade zu unterrichten, auf welchen sich die lichtbiologische Forschung bewegt. Nicht die rerschiedenen Arten der $L i c h t b e h a n d l u n g$ selbst, sondern vielmehr ihre Grundlagen, die in den verschiedenen Gebieten naturwissenschafticher Erkenntnis wurzeln, bilden den Gegenstand unserer Betrachtungen." 


\title{
Die physikalisch-chemischen Eigenschaften der Legierungen
}

\author{
Von Bernhard Dessau, a. o. Professor der Physik \\ an der Universität Perugia. Mit 82 Abbildungen im \\ Text und auf 3 Tafeln. VIII, 208 S. 1910. Geh. \\ M7.-, geb. M8.-.
}

\begin{abstract}
Inhaltsverzeichnis. I. Einleitung. - II. Allgemeineg uber Zweistoff * з у в teme. §1. Heterogene Gleichgewichte. §2. Gegenseitige Löslichkeit zweier Stoffe. 3. Erkaltungs- und Erwäruungskurven. Schmeladiagramme eiues Zweistoffoystems ohne chemische Verbindungen und polymorphe Umwandlungen, mit vollständiger Mlischbarkeit der Komponenteu im flüssigen, vollständiger Nichtmischbarkeit im kristallisierten Zustaude. \$4. Die Kompoueuten des Systems bilden uniteinander eine unzersetzt schmelzbare chemische Verbindung, die im kristallisierten Zustaude mit den Komponenten nicht misclibar ist. $\S 5$. Die Verbiudung schmilzt unter Zersetzung. § 6. Die Komponeuten des Systems bilden keine chemische Verbindung, sind aber sowohl im flussigen wio im kristallisierten Zustande in allen Verluältuissen miteinander mischbar. §7. Die Kompouenten sind im kristallisierten Zustande schon bei der Schmelztemperatur nur beschränkt ineinauder löslich. § 8. Beschränkte Mischbarkeit im tiussigen Zustande. \$ 9. Polymorphe Umwandlungen. - III. Untersuchungsmethoden. \$1. Thermische Analyse. § 2. Netallographie. §3. Dilatometrische und kalorimetrische Methoden. IV. Binäre Legierungem. \$1. Legierungen ohne chemisclie Verbindung der Komponenten. § 2. Binäre Legierungen mit Verbindungen. §3. Verbindungsfihigkeit und Isomorphismus der Metalle. - V. Ternäre Legierungen.- VI. Diegewerblich wichtigsten Legieruugen. \$1. Eisen und Kohlenstoff. §2. Legierungen des Kupfers. - VIr. Die physikalischen Ligenschaften. \$1. Nechanische und thermische Eigenschaften. §2. Elektrische Leitfäligkeit. §3. Der Magnetismus der Legierungen. \$ 4. Elcktrolytische Lösungstension und elektromotorische Kraft. - Register.
\end{abstract}

\section{Aus dem Vorwort.}

"Die Zahl der Untersuchungen ïber die Konstitution und die Eigenschaften der Legierungen ist in den letzten Jahren so sehr angewachsen, daß der Überblick für denjenigen, der sich nicht speziell mit dem Gegenstande beschäftigt, jmmer schwieriger wird. Und doch bietet gerade dieses Kapitel der phiysikalischen Chemie niclit uur für die Chemiker und Technolngen, von denen die wissenschaftliche Erforschung desselben in erster Linie betrieben wurde, sondern auch für den Physiker ein hervorragendes Interesse. Der Versuch, das einschlägige Material zusammenfassend darzustellen, bedart darum kaum einer Rechtfertigung. Der jetzige Zeitpuult erschien hierfür um so gecigneter, als die Klarlegung der Konstitution der binären Legierungen dank den Arbeiten Tammanns und seiner Schüler gegenwärtig zu einem gewissen Abschlusse gediehen und damit auch für das Studium der Legierungen von mehr als $z$ wei Komponenten, sowie für die systematische Bearbeitung des Zusammenhanges zwischen den verschiedenen Eigenschaften und der Konstitution der Lcgierungen erst die rationelle Grundlage gewonnen ist. In dieser Hinsicht mag die vorliegende Arbeit auch zu weiterer Forschung anregen, wenngleich begreiflicherweise die Originaluntersuchungen weder alle berücksichtigt, noch in Form von Literaturangaben sämtlich erwähnt werden konnten. Vollständigkeit wurde nur insofern angestrebt, als die verschiedenen Typen, denen man bei der Untersucliung der lionstitution der Legierungen begegnet, an charakteristischen Beispielen erläutert wurden..." 


\title{
Die elektrische Fernübertragung von Bildern
}

\author{
Von Dr. Robert Pohl, Assistenten am Physikalischen \\ Institut der Universität Berlin. Mit 25 Abbildungen. \\ VIII, 45 S. 1910. Geh. M 1.80, geb. M 2.50.
}

Inhaltsverzcichnis. Einleitung. - 1. Kapitel. Die Mögllchkejten dor elektrischen Zeichenubertragung. - 2. Kapitel. Über synchrontsmus. Zerlegung der Bilder in Flächenelemente. Uhrwerke. Casellis Pendelsynchronisierung. Nehenschlußmotore mit Zentrifugalregulatoren. Synchronisierung des Hughes-Apparates. Synchronisierung mit Korrektionsströmen, - 3. Kapitel. Kopiertelegraphen fitr өinen Leitungsdraht. Allgemeine Ausfürung. Bakewells Kopiertelegraph. Casellig Pautelegraph. Kopiertelegraphie mittels elektriscber Wellen. - 4. Kapitel. Kopiertelegraphen fir zwei Leitnagsdrähte. Telautographen. - 5. Kapitel, Appa. rate fur elektrische Fernphotographie (I. Teil). Unterschied ron den Kopier. telegraphen. Einteilung der Apparate. Die Kmpfangsapparate. Sender flir Reliefbi!der. Herstellung der Reliefs. Benutzung olektrischer Wellen - - Kapitel. A p parate fiur B]ektrische Fernphotographie (II. Teil). Sender mit Selenzellen. Selen und Selenzellen. Selentrigheit und Kompensation. Erste Versuche mit Selensendern. Apparat Bidwells. Empfänger Korns. - 7. Kapitel. Vergrößerung der Ǘtertragungs. seschwindigkeit.

\section{Vorwort.}

"Die große Beachtung, die das Problem der elektrischen Fernübertragung von Zeichnungen und Bildern in weiteren Kreisen gefunden liat, ließ es den Herausgebern der $2 W$ issenschaft wünschenswert erscheinen, eine Darstellung der bisherigen Entwicklung des Problems in die Sammlung aufzunehmen. Für eine derartige zusammenfissende Darstellung ist die größte Kürze geboten... Es liegt in der Natur des/Problems, daß sich die Ausfülırungen zum Teil auf technischem Gebiete bewegen müssen. Doch babe ich mich bemüht, alle rein konstruktiven Einzelheiten auszuschalten, wiewohl in diesen zurzeit die eigentlichen praktischen Schwierigkeiten liegen und gerade in der technischen Durcharbeitung der Fortschritt dieser im Prinzip schon seit vielen Jahrzehnten bekannten Apparate zu suchen ist."

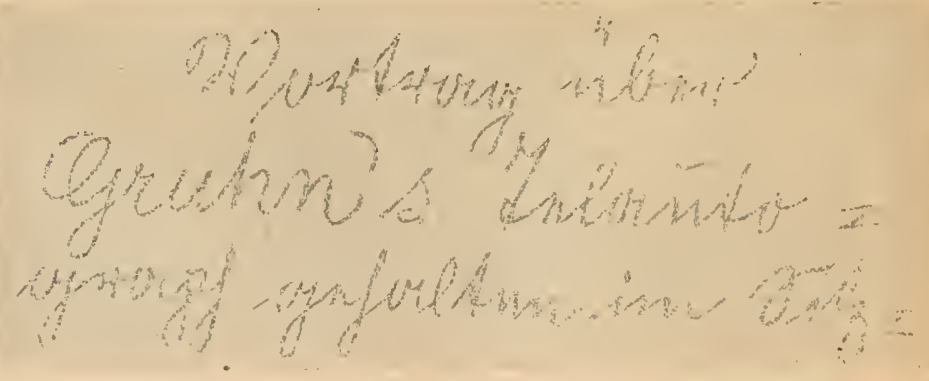




\title{
Die elektrischen Erscheinungen \\ in metallischen Leitern (Leitung, Thermoelek- trizität, Galvanomagnetische Effekte, Optik)
}

\author{
Von Dr. K. Baedeker, a. o. Professor an der Uni- \\ versität Jena. Mit 25 Abbildungen. VIII, 146 S. 1911. \\ Geh. M 4,-, geb. M 4,80.
}

\begin{abstract}
Inhaltsverzeichnis. Einleitung. Wesen der metallischen Leitung. Übersicht über die behandelten Erscheinungen. Prinzipien der theoretischen Behandlung. Elektronentbeorie der metallischen Leitung. Einfuhrung des $\mathrm{Maxwellschen} \mathrm{Ver-}$ teilungssatzes nach Lorentz. Elektronenemission glühender Körper. - 1. Kapitel. Die Hlektrizitätsleitung in Metallen. Allgemeines. Elektronentheorie der Leitung. Nessung der Leitfähigkeit. Beobachtungserg bnisse über das elektı ische Leitrermögen. Wirkung der Temperatur auf dıe Elekt izitätsleitung der reineu Metalle. Die Abbüngigkeit des Leitvermögens von der Temperatur bei schlechten Leitern. Wirkung des Diucks auf das elektrische Leitvermögen. Veränderung des Leitvermögens beim Wechsel des Aggregatzustands. Leitfäl,igkeit bei Modifikationeänderungen. Die Elektrizitätsleitung in Legierungen. Legieruugen mit gegenseltiger Löslichkeit der Kompouenten. Legierungen, welche Verbindungen enthalten. Wirkung der Temperatur auf das Leitvermögen der Legierungen. Zur 'Theorie der Leitung in Legiorungen. 2. Kapitel. Die Wärmeleitung in Metallen. Elektronentheorie der Wärmeleitung. Beobachtuug des Wärmeleitvermögens und des Leitverhältnisses. Spezielle Beobachtungseruebnisse über Wärmeleitung und Leitverhältnis, - 3, Kapitel. Die thermoelektrischen Erscheinungen. Die Messung thermoelektrischer Kräfte und ihre Ergebnisse. Thermoelektrizität der Legierungeu und Verbiudungen. Einfluf des Druckes auf die thermoelektrische Kraft. Der Peltiereffekt. Der Thomsoneffekt. Thermodynamiache Behandlung der thermoelektrischon Erscheinungen. Die Elektronentheoricn der Thermoelektrizität. - 4. Kapitel. Die galvanonagnetischeu und thormomagnetischon Erscheinuugen. Allgemeines über Transversaleffekte. Dio Beobachtung der Transversaleffekte und ihre Ergebnisse. Die Longitudinaleffekte. Wechselstrum-Gleichstromeffekt am Wismut. Znr 'Theorie der galvanomagnetischen Erscheinungen. - 5. Kapitel. Optische Eigenschaften der metallischen Leiter. Die optisclien Konstanten der Metalle. Theorie der elektromagnetischen Wellen in Leitern. Elektronentheorie der langwelligen Metallstrahlung von H. A. Loreutz. Die Keobachtung der Hmission und Reflexion der Metalle im langwelligen Spektrum. Die Dispersion der Metalle.
\end{abstract}

\section{Aus dem Vorwort:}

${ }_{\pi}$ Die elektrischen Eigenschaften der metallischen Leiter finden sich in den Lehrbüchern in der liegel an verschiedenen Stellen zerstreut untergebracht. Die rasche Entwickelung der Elektrizitätslehre, insbesondere die der Elektronenlehre im letzten Jahrzehnt gab auch auf diesem Gebiete eine solche Erweiterung des Tatsachenmaterials und eine so große Reihe gemeinsamer Gesichtspunkte, dab die in diesem Buche unternommene zusammenfassende Darstellung berechtigt erschen. Für die gewählte Darstellung war es wesentlich, daß die Theorie noch nicht in gleichem Naße, wie in anderen Gebieten die Grundlage und den Zusammenhang der Erscheinungen zu geben beanspruchen kann. An zwei Stellen wurde etwas mehr auf Einzelheiten eingegangen: bei der Richardsonschen Theorie der Elektronenemission durch glühende Leiter, die ich zum Teil als Grundlage für eine neue Theorie der Thermoelektrizität benutzte, und in diesem letzteren Ka Kapitel selbst ... Die experimentellen Ergebnisse des behandelten Geliets sind ausfïhrlicher wiedergegeben. Hier wurde eine gewisse Vollständigkett, besonders in den Zahlenangaben, erstrebt... Auch wurde Wert daraut gelegt, besonders die in den verbreiteten Handbüchern und Tabellen nicht enthaltenen Zahlen wiederzugeben ..." 


\title{
Grundlagen
}

\section{der praktischen Metronomie}

\author{
Von Prof. Dr. Karl Scheel, Mitgl. der Physikalisch- \\ technischen Reichsanstalt. Mit 39 Abbildungen. XII, \\ 168 S. 1911. Geh. M 5,20, geb. M 6,-.
}

Inhaltsverzeichnis. Einleitung. §1. Maroinheiten. §2, Einheit der Zeit. $\S 3$. Eiuheiten der Länge und der Masse. §4. Aufgaben der Metronomie. §5. Ältere Einheiten für Länge und Maße. $\S 6$. Vereinheitlichung des Maßsystems. Das Meter. § 7. Das Kílosramm. § 8. Das Raummaß. Tiubikdezimeter und Liter. § 9. Ausbreitung des metrischen Maßsystems. \$10. Tätigkeit des Bureau international des Poids et Mesures. - I. Abschnitt. Längenmessungen. $\S$ 11. S.richmabe und Endmaße. \$12, Einfache Längenmessungen an Strichmalien. $\$ 13$. Mikroskop. § 14. Nonisus. $\S 15$. Selıraubenmikromcter. § 16. Okularmikrometer. $\S 17$. Komparator. § 18. Transversalkomparator. \& 19. Kathetometer. § 20. Longitudinalkomparator. \$21. Schraubenteilmaschine. §22. Herstellung von Teilungen, §23. Untersuchung von Teilungen. § 24. Fehler und Korrektion. § 25. Bestimmung der inneren Teilungsfehler nach der Methode von Hansen. $\$ 26$. Erweiterte Hausensche Methode. \$27 Methode des Durchiiebens (Thiesen, Leman). § 28. Fehler von Schrauben. $\$ 29$. Fortschreitende Schraubenfehler. \$30. Perjodische sehraubenfehler. $\S 31$. Formen der Endmaße. \$ 32 . Anschluß der EndmaLe an Strichmaße. \$33. Vergleichung und Unterteilung von Hndmafien. \$34. Mikrometerschraube, § 35 . Melimaschinen. $\$ 36$. Sphärometer. § 37. Messung der Höhenunterschiede von Quecksilbesräulen. - II Abschnitt. Einfluß der 'Temperatur bei den Längeumessungen. Wärmea us dehnung. §38. Material der Maßstibe. §39. Normaltemperitur eimes Maßstabes. $\S 40$. Temperaturskala. $\S 41$. Quecksilberthermometer. $\S 42$. Platinthermometer. \$43. Thermoelement. \$ 44. Messung der Warmeausdehnung auf dem Konlparator. $\S 45$. Beispiel für eine Ausdelınungsbestimmung. § 46. Messung der Wärmeausdehnung nach der Interferenzmethode. $\S 47$. Messung der Ausdeliunug durch Wägung. § $\$ 8$. Bäder konstanter 'I'emperatur. - III. Abschnitt. Massenmessungen. $\S 49$. Allgemeine Aufgabe. $\S 50$. Die gleicharmige Wa spiele von Wagenkonstruktionen. §52. Gleichgewichtslage der Wage. §53. Wägung durch Substitution nach Borda, \$54. Wägung durch Vertauschen nach GauB. \$55. Vollständige Wägung. § 56. Vergleichung mebrerer nalıe gleicher Massen. \$57. Massenuormale. \$58. Massensätze und ihre Etılannierung. - IV. A bschnitt. Einflub äuberer Verhältnisse auf die Wägungen. Ihre Berücksichtigung. §59. Änderung der Sclıwere mit der IHöhe. § 60. Eiuflu@ der Luftaichte. \$ 61. Bestimmung der Luftdichte aus meteorologischen Beobachtungen. \$ 62. Messung der Lufttemperatur. $\S 63$. Messung des Luftdrucks. $§ 64$. Messung der Spannkraft des Wasserdampfes. \$ 65 . Experimentelle Bestimmung der Luftdichte während der Wägung. § 66 . Wägungen im Takuum. § 67. Aufbau von Massen beliebigen Volumens. - V. Abschuitt. Raummessungen. \$68. Volumenbestimmung durch Linearmessung. $\$ 69$. Pyknometer. $\S 70$. Volumen aus Masse und Jichtigkeit. $\S 71$. Volumenbestimmung durch Wägung in einer Fiüssigkeit. § 72. Dichte des Wassers. \$ 73. Ausfübrumg hydrostatischer Wägungen. \$74. Bestimmung der kubischen Ausdehnung eines hörpers durch hydrostatische Wägung. \$ 75. Bestimmung eines Gefälinhalts durch Wägung. \$ 76. Bestimmung der Wärmeausdchnung cines Hohlkörpers durch Wägurg. § 77. Dichte des Quecksilbers. \$78. Volunenometer. $\S 79$. Bestimmung äuferer Volumina mit den Volumenometer. - VI. Absehnitt. Sicherungcn des metrischen Mialisystems. § 80 . Interferenzerscheinungen, §81. Auswertung des Meters in Lichtwellenlängen. Versuche von II iche Is on. $\$ 82$. Versuche von Benoit, Fabry und Perot. § 83, Angchluß der Masseneinheit an dic Längeneiuheit. 


\title{
Die Wissenschaft
}

\section{Vergleichende Mond- und Erdkunde}

\author{
Von Dr. Siegmund Günther, Professor an der \\ Technischen Hochschule München. Mit 28 Textabbild. \\ und 4 Tafeln. XI, 193 S. 1911. Geh. M 5,-, \\ geb. M 5,80.
}

Inhaltsverzeicbuis. I. Abschnitt. Die Pluralitätshypothesen im allgemeinen. - II. Abschnitt. Die Berechtigung einer vergleichenden Oberflächenkunde von Erde und Mond. - III. Abschnitt. Vergleiche zwischen beiden Weltkorpern h rorteleskopischer Zeit. - IV. Abschnitt. Galilei und Kepler. - V. Abschnitt. Die Ausbildung der Selenographie im 17. and 18. Jahrhundert. - VI. Abschnitt. Die lunare Pluralitätshypothese. - VII. Abschnitt. Die teleskopische Mondbeobachtung im 19. und beginnenden 20. Jahrhundert. - VIII. Abschnjtt. Die Mondphotographie und die physikalische Mondforschung uberhaupt. - IX. Abschnitt. Die Mondoberfläche auf Grund der Gegenwarterkenntnis betrachtet. - X. Abschnitt. Der lunare Vulkanismus. - XI. A bschnitt. Tektonische Dislokationen auf dem Monde. - XII. A bschnitt. Die Streitfrage nach den rezenten Veränderungen auf dem Monde. - XIII. Abschnitt. Mondoberfäche und Meteorkŏrper. XIV.Abschnitt. Zusammenfassender Rtukhlick. - Namensverzeichnis.

\section{Aus dem Vorwort.}

„Die vorliegende Schrift ist dazu bestimmt, einen Gedanken weiter auszuführen, welchen der Verfasser vor einer Reihe von Jahren (1899) in der "Umschau" kurz skizziert hat. Von den ältesten Zeiten an soll der Gedanke, im Monde sei "eine zweite Erde" anzuerkennen, durch die Jahrhunderte verfolgt werden, um zuletzt zu zeigen, daß in der Tat mit gutem Rechte ein Vergleich zwischen Mond- und Erdkunde gezogen werden kann, der in sich volle Berechtigung besitzt, sobald man nicht in den freilich sehr gefährlichen und wiederholt begangenen Irrtum verfällt, Analogie mit Identität zu verwechseln. Eine große Schwierigkeit bestand darin, aus der so ungemein reichhaltigen und verzweigten Literatur nur diejenigen Materien auszuheben, welche für unseren Zweck unmittelbar bedeutsam sind, während eine Fülle einschlägiger Veröffentlichungen nicht für den Geographen, sondern ausschließlich für den Astronomen von Wichtigkeit ist. Inwieweit dieses Streben vom Erfolge gekrönt war, das zu beurteilen muß anderen Stellen überlassen bleiben. Daß Puiseux' oft zitiertes Werk sich nach Ziel und Inhalt mit dieser Arbeit nur sehr teilweise deckt, zeigt die Lektüre....." 


\title{
Das Relativitätsprinzip
}

\author{
von Dr. M. Laue, Privato'ozent für theoretische Physik \\ an der Universität München. Mit 14 Abbildungen, $X$, \\ 208 S., 1911. Geh. M 6,50, geb. M 7,20.
}

Inhaltsverzeichnis. I. Die Problemstellung. \$1. Iie Relativitätsprinzipe der klassisclien Mechanik und der Elektrodynauik. § 2. Die empirischeu Grundlagen für die Elektrodyuamik bewegter Körper. Induktion. Wilsonscher Versuch. Rowlandscher Versuch. Versuche von Róntgen und Eichenwald. Fizeauscher Ver. such. Aberration. Dopplereffekt. Micbelsonscher Versuch. Andere Versuche über den Einfluß der Erdbewegung. Dynamik des Elektrons, - II. Die älteren Theorien der lilektrodynamik bewegter Korper. \$ 3. Historische Übersicht. \$4. Lie 'Theorie von Heinrich Hcrtz: a) Ruhende Körper. h) Bewegte hörper; c) Der Wilsonsche Versuch; d) Dcr Eicbenwaldsche Versuch; e) Der Hizeansche und II chelsonsche Versuch; f) Der Induktionsvorgang; g) Die Erbaltung des Impulses. 85. Die Elektroneutheorio. - III. Die Relativitätstheorie, kinematischer Te il. \$6. Die Loreutz-Transformation. \& 7. Die Linsteinsche Kinematik. \$8. Minkowskis geometrische Interpretation der Lorentz-Transformation. §9. Die Lorentz-Transformation als imagiuäre Diehung. - IV. Weltvektoren und -tensoren. \$ 10. Vierer - uud Sechservektoren: a) Vierervektoren; b) Sechservektoren. \$ 11. Die algebraischen Vektoroperationen: a) Additiou und Subtraktion; b) Die $;$ kalare Iultiplikation; c) Vektorprodukte. § 12. Vektorielle Differentialoperatiouen. $\$ 13$. Weltensoren. - V. Die Hlektrodynamikdes leeren Raumes nach dem Relativitätsprinzip. \$14. Die 'Transformation des elektromagnetischen Feldes im leeren Raume. 15. Die 'Traneformation der Krafteichte, Energie und Impulssatz: a) Dio Viererkraft; b) Der Welttensor $T$; c) Der Energiesatz; d) Die Erhaltung des Impulses; e) Die Erhaltung des Drehimpulses; f) Transformation der Energie, des Energiestromes und der Spannungen. \$ 16. Amwendungen: a) Aberration und Wop plersches Prinzip; b) Die Retlexion am bewegten Spiegel. §17. Gleichiörmige Bewegung geladener Körper: a) Das elektromagnetische Feld; b) Das Feld eines bewegten Elektrons; c) Die Rückwirknng des Feldes auf bewegte Träger von Ladungen; d) Energio und Impuls des Feldes; e) Beispiel des kugelförmigen Elektrons; f) Der 'Trou ton - Noble sche Versuch. $\$$ 18. Ungleichförmig bewegte Ladungsträger: a) Das Viererpotential; b) lie retardjerten Poteutiale ; c) Die Hyperbelbewegung; d) Das Viererpotcntial bei der Hyperbelbewegung; e) Das elektromagnetische Feld bei der Hyperbelbewegung; f) Die Rückwirkung des Feldes auf das Elektron; g) Näherung für kleine keschleunigung. - VI. Die Min kowskische lilektrodynamik der ponderablen Körper. \$19. Die Transformation der Feldgleichungen I bis IV: a) Die Feldvektoren (5, 5 , 5 , , B; b) Leitungsund Konvektionkstrom; c) Die Invariauz der Elektruzitätsmenge. \$20. Die Transformation der Gleiclungen $\nabla$ bis VII: a) Jer Zusammeuliang zwischen (5, $5,5,13$; b) Elektromotorische Kraft und Leitungsstrom. \$ 21. Anwendungen: a) Das Ohmsche Gesetz; b) Induktion; c) Grenzbedingungen; d) Der W ilson scbe Versuch; e) Der Ejchenwaldsche Tersuch. \$22. Energie und ponderomotorische Kraft: a) Die Unzulänglichkeit der Maxwellschen Theorie; b) Die 'Trausformation beliebiger ponderomotorischer Kräfte; c) Die Bedeutung der Komponenten des Welttensors $T$; d) Anwendung auf die Elektrodynamik; e) Die Joulesche Wärme. § 23. Der Strahlungsdruck. - VII. D y n mik. \$ 24. Die mechanische Träglieit als Wirkung der Energie: a) Zweck und Ausgangspunkt der Betrachtungen; b) Impuls und Energie; c) Diskussion von XXVI. \$ 25. Impuls, Energie und Spannung in jhrer Abhängigkeit von der Geschwindigkeit und dem inneren Zustand: a) Ableitung der Gleichungen; b) Liskussion; c) Vergleich mit der klassischen Mechanik. \$26. Die Bedeutung der dynamischen Viererkraft $F$ und die absoluten und relativen Spanuungen. $\$ 27$. Beispiele für die quasistationäre, adiabatische, sopieistische Dynamik: a) Der Massenpunkt; b) Die Dynamik einer elektrisch geladeueu Kugel; c) Körper mit beliebigen spannungen; d) Vollständiges statisches system. \$ 28. Thermodyuamik: a) Trausformation der Entropie; b) Transformation der Temperatur; c) Bestätigung von XXX; d) Das dynamische Potential $\mathrm{H}$; e) Isotherm-isochore Dynamik; f) Das P'rinzip der kleiusteu Wirkung. \$ 29. Die Dynamik der Hohlraumstrahlung: a) Die hohlraumstrahlung in der Ruhe; b) Die Hohlraumstrahlung in gleichförmiger Bewegung; c) Isotherm-isochore Dynamik; d) Historische Bemerkungen. \$30. Rückblicke und Ausblicke. - An liang. a) (ieometrische Bezeichnungen; b) Vektor - und Tensorbezeichnungen; c) Die verschiedenen Arten der Keitdilferentiation; d) Bezeichnung und Maßsystem der physikalisclien Großen. Literatur. Namenregister. Sachrepister. 


\title{
Das Problem des absoluten Raumes u. seine Beziehung zum allgem. Raumproblem
}

\author{
Von Aloys Müller. X, 154 S. 1911. Geheftet \\ M 4,-, gebunden M 4,80.
}

lulualtsverzeichnis. Einleitung. Konstruktion des erkeuntnistheoretisch neutraleu Weltbildes. - Erster Teil. Logisch-physikalische 'Theorie des absoluten Raumes. I. Das phoronomische Weltbild. Definition dieses Weltbildes und der Begrifte absolnt und relativ. Phoronomisches Relativitätsprinzip. Absolute Bewegung für die Mechanik umbrauchbar. Weitergehende positivistische Ansichteu. Stelluug des phorononuschen Bildes zum Begriff des absoluten Raumes. II. Die Dynamik des phoronomischen Weltbldes. Unbestimmtheit dynanischer Grundbegriffe innerhalb des phoronomischeu Bildes; Aquivalenz der geometrischen und dynamischen Beziehungen. III. Die Versuche zur Konstruktion des dynamiaclen Weltbildes: Der ers:e Weg. Zwei Wege möglich. Mach als Typus des ersten Weges. Die drei Nuancen, die $M$ a ch ihm gegeben hat, sind teils nur vorläufig brauchbar, teils überhaupt unaunehnbar. IV. Die Versuche zur Konstruktion des dynamischen Weltbildes: Ner zweite Weg. Die beste Durchfülırung ron L. Lange. Definition der gleichen Zeitintervalle uach Neumann. Definitiou des Intertialsystems; notweudige Bestandteile des Begriffsinhaltes. Tatsäcbliche Festlegung des Inertialsystems nicht streng nöglich. V. Inertialsystem und absolut $r$ liaum. Ideales inertialsystem 1. ohne unmittrlbaren Zusammenhang mit dem tatsächlichen Inertialsystem; 2. von demselben Realitätscharakter wie die laumnomente der Körper, - Begrıff des Neumannscheu Körpers gegen Einwände verteidigt, aber nicht in dem Neumannschen sinne brauchbar - : 3. deshalb identisch nit dem Begritfe des absoluten Raumes. VI. Lozik des absoluten Raumes. Unterscheidung des phoronomisch-dynamischen vom physikalischen Begriff des absoluten Kaumes. Absolute Bewegung. Grenztall der Kelativbewegung. Eigentünlicher Charakter der Transzendeuz des absoluten Kaumes. Verweehslung von nabsolut" mit nobjektiv" und anderen Bigrifien. Begriff des starren Ranmes. Das relativi-tische bild als notwendige Konsequenz des abolutistischen. VII. Das Trägheitsprınzip und die 'Trägheitswirkungen. Scheidung in phorunomisch-dynamische und physikalische Fassung des Trägheit.prinzips. Verlältnis zu den beiden Begriffen des absoluten Raumes. 'Irägheitsprinzip in relativer fassung. Der absolute Charakter der Rotation. Trägheitsprinzipien in anderer Form. Fegriff der Urduung der Relativsysteme. - Zweiter Teil. Philosophische Theoriedes absoluten Raumes. I. Die allgemein-logische Begründung des absoluten Raumes, Detinition der Realität der Relativbewegung. Unaufteilbarkeit der realen Distanzäuderung. Prinzip der konkreten Bestimmtheit als Konsequenz aus dem Identitätsprinzip. Absolute Aufteilung als logisch gefordert von dem Prinzip der koukreten Bestimmtheit; nur das Dynamische teilweise willkürlich. - Gleichwirklichkeit des ptolemäischen und des kopernikanischen Weltsystems. - Kritik der Versuche von Heymans und Höfler. If. Metapbysik des absoluten Raumes. Kurze Begründung des idealrealistischen erkemntnis - theoretischen Standpunktes durch Kritik dos Idealismus und des Gegebenheitsstandpunktes. Objektives und subjektives a priori. Empirismus und Nativismus. Unabhängigkeit des Raumes von den Diugen. Der Raum als etwas objektiv für sich Bestehendes. Harmonisicrung der Begriffe des apriorischen und objektiveu Raumes. Abhängigkeit der Körper rom Raum. Metaphysischer Begritt des absoluten Raumes. Absolute Zeit. Vergleich mit den Newtonschen Begriffen. Hine merkwürdige Eigenschaft des absoluten Raumes und der absoluten Zeit. Uer Ram als Substanz. III. Die Grundlagen der Mletaphysik des absoluten Raumes in der modernen Physik. Verhältnis der physikalischen GrundJagen zu den metaphysischen Untersuchungen. Prinzip der Koustanz der Mlasse in dem bisherigeu Sinne ta!sch. Elektron. Elektromagnetisches Weltbild. Absolute Rube des Äthers. Charakter der Atherhypothese. Ersetzung des Äthers durch den Raum. Zeitlich sich fortpflanzende Fernkräfte. Fernwirkungstheorio und phänomenologischer Standpunkt. trsatz des Athers nötig. - Einsteinsches Prinzip - ein Rechunngsprinzip. - Das physikalische Weltbild an und für sich metaphysisch vieldeutig und ein Bild der phänomenalen Welt. - Konsequeuzen aus den physikalischen Darlegungen. - Driter Teil. Die nichtexklidischen Geometrien und der absolute $\mathrm{R}$ an m. Mehrdimensionale Geometrien. Der Raum hat keine Dimensionen. Begriff der nichteuklidischen Käume. FerbäItnig des Erfahrungsraumes zum enklidischen und nichteukJidischen Charakter. Die nichteuklidischen Rälime absolute Räume. Begriff der Absolutheit der Grölé im nichteuklidischen Raum. Gründe gegen die tatsächliche Existenz eines nichteuklidischen Raumes nicht stichhaltig. Die tatsächliche Fixistenz eines nichteuklidischen Raumes mit variablem Krümmung $\times$ ma L als Element in umserem Weltbild. - Schematische Übersicht uber die Raumtheorie. - SchluB. - Anhang. - Verzeichnis der zitierten Literatur. 


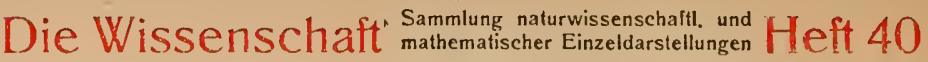

\section{Die Leuchtgaserzeugung u. die moderne Gasbeleuchtung (Preßgasbeleuchtung usw.)}

Von Fritz Schmidt, Ingenıeur in Berlin. Mit $63 \mathrm{Ab}$ bildungen. VII, 86 S. 1912. Geheftet M 2,50, gebunden $M$ 3,20.

\footnotetext{
Inhaltsverzeichnis. A. Einleitung. - B. Entwickelung der Beleuchtungstechnik. Frfindung des Leuchtgases. Erste Anwendung des Leuchtgases in England und seine Einführung in Berlin durch die Engländer. Berlin als charakteristisches Beispiel für die Entwickelung der Leuchtgasindustrie in einer Stadt. Jährliche Gasproduktion der Gaswerke verschiedener grölerer Städte Preulens. - C. Heratellung des Leuchtgases. Steinkohle; ihre Entstehung, ihr Vorkommen und ihre Bestandteile. Retortenöfen; horizontale, schräq liegende und vertikale Öfen. Rostöfen, Generatoröfen und Halbgeneratoröfen, Geblisscmaschineu. Luft- und Wasserkuhler. Teerscheider ron Pelouze und Andoin und Teerwäscher von Drory. Rohes Leuchtgas. Reinigung des Gases. Naphtaliuwäscher. Cyanwäscher. Ammoniakwäвcher. Eisenreiniger. - D. Das zum Gebrauch fertige Leuchtgas. Gereinigtes Leuchtgas. Heizwert des Leuchtgases. Brennwertmesser von Prof. Junker. Mischgas. Gasmesser. Gashehälter. Druckregler. Manometer. Leitungsnetz. Wasserabscheider. - E. Anwendung des Leuchtgases. Leuchtliraft des Gases. Lichtmesser. Brenner. Stehendes und hängendes Gasgluhlicht. Betriebskostenvergleich zwischen Gasgluhlicht, elektrischem Licht und Petroleumlicht. Zundungen. - F. Starklicht. Allgemeines. Preßgasanlagen. Preßluftanlagen. Anlagen mit gepreßtem Gas- und Iuftgemisch. Millenniumlicht. Pharoslicht. Keithlicht. Selaslicht. Prelgas - und Preßiluftlampen. Zandungsvorrichtungen für Prefgas- und Prefluftlampen. G. Schlu \&wort.
}

\section{Vorwort.}

Heftiger denn je ist der Kampf zwischen dem Steinkohlengas und dem elektrischen Strom als Beleuchtungsmittel entbrannt; die neuesten Erfindungen auf diesem Gebiete spornen zum gegenseitigen Wettkampf an. Das vorliegende Bändchen versucht eine gemeinverständliche und übersichtliche Darstellung der geschichtlichen Entwickelung und des augenblicklichen Standes der Leuchtgaserzeugung, sowie der Anwendung des Steinkohlengases als Beleuchtungsmittel zu geben. Eine besondere Berücksichtigung hat die moderne Anwendung des Steinkohlengases als sogenannte Preßgas - und Preßluftbeleuchtung gefunden, Das kleine Buch dürfte allen denen willkommen sein, welche sich in kurzer Zeit einen Überblick über die Entwickelung, Erzeugung und moderne Anwendung des so wichtigen Beleuchtungsmittels verschaffen wollen. 


\title{
Die Wissenschaft Sammlung naturvisisenschathth und Heft 41
}

\section{Der Weltäther}

\author{
Von Sir Oliver Lodge. Übersetzt von Hilde \\ Barkhausen. Mit 17 Abbildungen und 1 Tafel. \\ VII, 107 S. 1911. Geh. M 3,-, geb. M 3,60.
}

Inhaltsverzeichnis. Einleitung. Allgemeines und Historisches. - I. Der Lichtäther und die moderne Theorie des Lichtes. - II. Der Ather im Weltenraume als verbindendes Medium. - III. Der EinfluB der Bewegung bei verschiedenen Erscheinungen. - IV. Experimentelle Versuche über den Äther. - V. Ein spezieller Versuch tiber die Viskosität des Äthers. - VI. Die Dichte des Äthe.s, - VII. Weiteres uber die Dichte und Energie des Athers. - VIII. Äther und Materie. - IX. Kraft im Äther. - X. Allgemeine Theorie der Aberration. - Anhang. Uber Gravitation und Ätherspannung. Berechnungen mit Hilfe der Ätherdichte. Fresnels Gesetz als Spezialfall einer allgemeinen Potentialfunktion.

\section{Ankündigung.}

Lodge entwickelt in diesem Buche die Ansicht, die er sich auf Grund eigener und fremder Experimente und auf Grund theoretischer Uberlegungen über die Natur des Weltäthers gebildet hat: die Ansicht, daß der Äther eine kontinuierliche, inkompressible, ruaende Ursubstanz sei, mit einer Dichte . von $10^{12} \mathrm{gr} / \mathrm{ccm}$ und einer inneren Energie von $10^{33} \mathrm{Erg} / \mathrm{ccm}$. Er wendet sich sowohl an den Laien, dem er bei Vermeidung aller Mathematik durch einfache, handgreifliche Beispiele die schwierigen Begriffe zu veranschaulichen sucht, als auch an den Physiker, der eine kritische Zusammenstellung der bisher ausgeführten Versuche über die relative Bewegung zwischen Äther und materiellen Körpern und in den Schlußkapiteln eingehendere theoretische Erörterungen und Folgerungen findet. 


\title{
Wechselstromversuche
}

\author{
Von Dr. Anton Lampa, o. Professor der Physik \\ an der Deutschen Universität Prag. Mit 54 Abbildungen. \\ X, 176 S. 1911. Geh. M 5,-, geb. M 5,80.
}

Inholtsverzeichnis. Einleltúg. \& 1. Grundbegriffe. - Erstes Kapitel. Die Leitung des Wechselstromes. §2. Der einfache Stromkreis mit Widerstand und Selbstinduktion. § 3. Die Erzeugung von Feddersenströmen durch Kondeneatorentladung. §4. Der Impedanzversuch von Tesla. §5. Der Impedanzverbuch von P. H. Ey k mann. $\S 6$. Der Skineffekt. $\S \%$. Der einfache Stromkreis mit Wideretand, Selbstinduktion und Kapazität. - $Z$ weites Kapitel. Induktion und ponderomotorische Kräfte. § 8. Induktion durch einen von Wechselstrom durchflossenen Stromkreis. §9. Die ponderomotorische Kraft zwischen dem primären und sekundären Stromkreis. Versuche von Elihu Thoms on und V. V. Lang. $\S 10$. Der sekundare Stromkreis eine Kapazität. v. Lang s Methode der Bestimmung der Kapazitä mit der Wage. § 11. Bestimmung der Selbatinduktion mit der Wage nach W. Peukert. \$ 12. Der Resonanzversuch ron Lodge. - Drittes Kapitel. Das magnetische Drehfeld. § 13. Das einfache Drelifeld. \$14. Die Herstellung des einfachen Drehfeldes mit Einphasenstrom. § 15. Herstellung des einfachen 1)rehfeldee durch Dreiphasenstrom. \$16. Die Herstellung des einfachen Drehfeldes mittels Gleichstrom. 8 17. Demonstration eines maguetisclien Drelifeldes nach $R$ ubens-Bode. $\S 18$. Verauche im magnetischen Drehfeld. § 19. Die Größe des Drehungsmomentes. § 20. Rotationen im reinen Wechselfeld. \$21. Die Zerlegung eines Wechselfeldes in zwei Drehfelder. - Viertes Kapitel. Das elektrostatiache Drehfeld. § 22. Das elektrostatische Drehfeld. Anordnung ron Arno. \& 23. Die Drehfeldschaltung von Guye und Denso. Erete Anordnung von $\nabla$. Lang. \& 24. Zweite Drehfeldanordnung von v. Lang. \$25. Die Drehfeldanordnung von Lampa. \& 26. Versuche im elektrostatischen Drehfelde. \& 27. Theorie der Rotationserscheinungen im elektrostatischen Drehfelde. 828. Rotationen im einfachen elektrostatischen Wechselfelde. Versuche yon $\mathrm{Ch}$ Borel und v. Lang. \$29. Theorie der Rotationserscheinungen im Wechselfelde.

\section{Vorwort.}

Die nachfolgende Schrift beabsichtigt, ohne erschöpfend sein zu wollen, Wechselstromerscheinungen und Wechselstromversuche darzustellen, welche in erster Linie von physikalischem Interesse sind. Der Beschränkung im Inbalt steht die eingehende Durchrechnung der behandelten Probleme gegenüber. Eine gewisse Breite der Darstellung war hier Gebot, wenn dem weiteren Leserkreis, an welchen sich ${ }_{n} D i e$ Wissenschaft" wendet, gedient sein sollte. Die Beschreibung der zu den behandelten Versuchen nötigen Apparatur enthält Zahlenangaben, welche dem Experimentator ausreichende Orientierung für die Ausführung der Versuche gewähren. Das letzte Kapitel, „Das elektrostatische Drehfeld “, berühtt das interessante Problem der dielektrischen Hysteresis. Die physikalische Erforschung dieses Gebietes ist noch nicht zu abschließenden Ergebnissen gelangt. Es wurde darum ganz darauf verzichtet, auf die Rolle einzugehen, welche die dielektrische Hysteresis bei den in diesem Kapitel beschriebenen Erscheinungen allenfalls spielen könṇte. 
Curie, Madame S., Untersuchungen über die radioaktiven Substanzen. Ubersetzt und mit Literatur-Ergänzungen versehen von W. Kaufmann. 3. unveränderte Auflage. Mit 14 Abbildungen. VIII, $132 \mathrm{~S}$. 80 . 1904. (,Die Wissenschaft", Heft 1.) $163,-$, in Lnwdbd. 163,80 .

Fischer, Prof. Dr. Otto, Kinematik organischer Gelenke. Mit 77 Abbildungen. XII, 261 S. S . 1907. ("Die Wissenschaft", Heft 18.) $168,-$, in Lnwdbd. $169,-$ :

Greinacher, Dr. H., Über die Klassifizierung der neueren Strahlen. $14 \mathrm{~S}$. gr. 80. 1908.

16,- 60 .

Die neueren Fortschritte auf dem Gebiete der Radioaktivität. (Von Anfang 1906 bis Mitte 1908.) 47 S. gr. $8^{0} .1908$.

16 1,20 .

Rutherford, Prof. F., Radioaktive Umwandlungen. Ubersetzt von M. Lewin. Mit 53 Abbild. VIII, 285 S. 80. 1907. (,Die Wissenschaft", Heft 21.) $.168,-$, in Lnwdbd. 168,60 .

Thomson, Prof. J. J., Elemente der mathematischen Theorie der Elektrizität und des Magnetismus. Autorisierte deutsche Ausgabe von Prof. Gustav Wertheim. Nit 133 Abbildungen. XIII, 414 S. gr. S0. 1907. 16 8,-

Elektrizität und Materie. Autorisierte Ubersetzung von G. Siebert 2. verbesserte Auflage. Mit 21 Abbildungen. VIII, $116 \mathrm{~S} .8^{0} .1909$. (,Die Wissenschaft", Heft 3.)

.163 , 一, in Lnwdbd. .16 3,60 .

Die Korpuskulartheorie der Materie. Autorisierte Ubersetzung von G. Siebert. Mit 29 Abbildungèn. VIII, 166 S. $8^{\circ}$. 1908. (,Die Wissenschaft", Heft 25.) 165 , - in Lnwdbd. 165,80 .

Hofmeister, Prof. Dr. Fr., Leitiaden für den praktisch-chemischen Unterricht der Mediziner zusammengestellt. 4. neu durchgesehene und vervollständigte Auflage. VIII, $152 \mathrm{~S} .8^{0}$. 1911. $164,-$, in Lnwdbd. $164,80$.

Die chemische Organisation der Zelle. Ein Vortrag. 29 S. 80. 1901. $.16-, 60$.

Parker, Prof. T. Jeffery, Vorlesungen über elementare Biologie. Autorisierte deutsche Ausgabe von Dr. Reinold v. Hanstein. Mit 88 Abbildungen. XXI, 303 S. gr. $8^{\circ}$. 1895.

168 , 一.

Pauli, Dr. Wolfgang, Der kolloidale Zustand und die Vorgänge in der lebendigen Substanz. Vorgetragen in der morphologisch-physiologischen (iesellschaft am 13. Nai 1902. $32 \mathrm{~S}$. $\mathrm{S}^{0}$. 1902. $.16-, 60$. 


\section{Hanllbuch dep Physiononje des Mencehen}

Herausgegeben von W. Nagel

In fünf Bänden. Lex.-8 $8^{0}$

\section{Erster Band.}

Physiologie der Atmung, des Kreislaufs und des Stoffwe chsels, bearbeitet ron Chr. Bohr-Kopenhagen, H. Boruttau-Berlin, F. B. Hofmann-Innsbruck, G. F. Nicolai-Berlin, K. Oppenheimer-Berlin, R. Tigerstedt-Helsingfors. Mit 86 Abbildungen. X1V, 874 S. 1909. M 27,-, in Hlbfrzbd. $M 30,-$

\section{Zweiter Band.}

Physiologie der Drüsen, Physiologie der inneren Sekretion der Harn-, Geschlechts- und Verdauungsorgane, bearbeitet von H. Boruttau - Berlin, O. Cohnheim - Heidelberg, R. Metzner-Basel, W. Nagel-Berlin, E. Orerton-Lund, I. Pawlow-St. Petersburg, H. SellheimDüsseldorf, E. Weinland-München, O. Weiss-Königsberg. Mit 2 I $3 \mathrm{Ab}-$ bildungen und 3 Tafeln. XV, $102+$ S. 1907. $\mathscr{M} 32$, , in Hlbfrzbd. M 35, .

\section{Dritter Band.}

Physiologie der Sinne, bearbeitet von J. v. Kries-Freiburg i. Br., IV. Nagel-Berlin, K. L. Schaefer-Berlin, Fr. Schenck-Marburg, T. ThunbergUpsala, O. Weiss-Königsberg, O. Zoth-Graz. Mit 134 Abbildungen und 2 Tafeln. XVII, 806 S. 1905. M 22, 一, in Hibfrzbd. M 24,一.

\section{Vierter Band.}

Physiologie des Nerven- und Muskelsystems, bearbeitet von R. du Bois-Reymond-Berlin, M. Cremer-Cöln, M. ron Frey-Würzburg, O. Langendorff-Rostock, W. Nagel-Rostock, P. Schultz-Berlin, A. Tschermak-Wien, O. Weiss-Königsberg. Mit 184 Abbildungen und 2 Tafeln. XVIII, 992 S. 1909. $\mathscr{M} 32,-$, in Hlbfrzbd. $M$ 35, -

Fünfter (Ergänzungs-) Band.

Blut und Lymphe. Entoptische Erscheinungen. Nachträge. Sachregister. Bearbeitet von H. Boruttau-Berlin, A. LohmannMarburg, R. Metzner-Basel, T. Thunberg-Lund, O. Weiss-Königsberg. Mit 18 Abbildungen. X, 149 S. 1910. M 7,-, in Hlbfrzbd. $\mathscr{M}$ 9,- 
Verlag von Friedr. Vieweg \& Sohn in Braunschweig

\section{Prof. Dr. Julius Bernstein}

Die Kräfte der Bewegung in der lebenden Substanz. IV, 28 S. gr. So. 1902 .

$.11-, 80$.

Die mechanistische Theorie des Lebens, ihre Grundlagen und Erfolge. Rede, bei der Übernahme des Rektorats an der Universität Halle-Wittenberg am 12. Juli 1890 gehalten. IV, 26 S. gr. So 1890.

el,- 60 .

Die Vorbildung der Medizin-Studierenden im Himblick auf den Entwurf der neuen Prifungsordnung. IV, $25 \mathrm{~S}$. gr. So $.1899 . \quad$. H. - , s0.

\section{Allgemeine Vererbungslehre Von}

\section{Prof. Valentin Haecker}

2. vermehrte Auflage. Mit einem Titelbilde, 133 Fïguren im Text und 4 farbigen Tafeln. XII, 405 S. gr. $8^{\circ} .1912$.

. $10,-$, in Leinwandband $/ 111,--$.

\section{Lichtbiologie}

Die experimentellen Grundlagen der modernen Lichtbehandlung von

\section{Dr. Albert Jesionek}

VIII, 177 s. 1910. H 4,-, in Leinwandband th 4,80. 


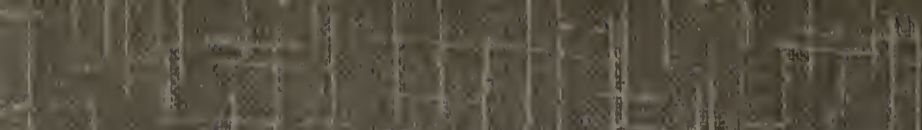

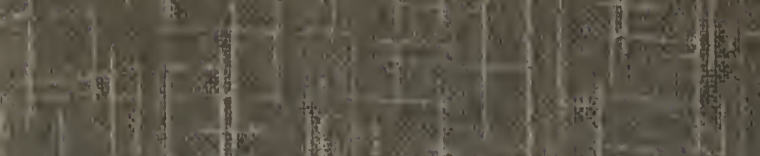
(1. (19)

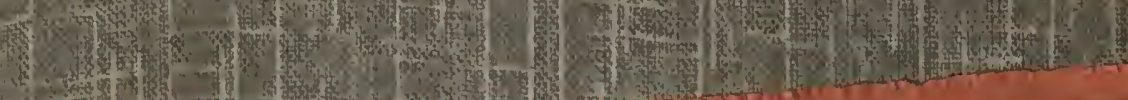

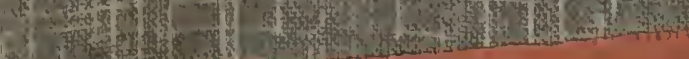

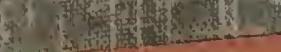

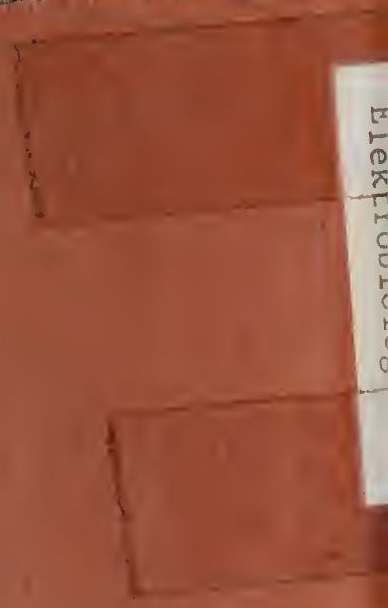

